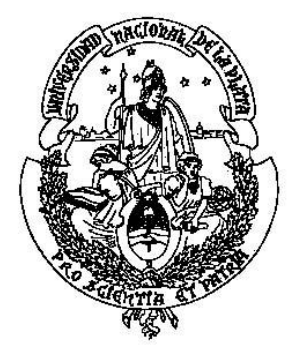

UNIVERSIDAD NACIONAL DE LA PLATA

FACULTAD DE CIENCIAS EXACTAS

DEPARTAMENTO DE FÍSICA

\title{
Estudio de las propiedades ópticas de materiales nanoestructurados y aplicaciones
}

\author{
Trabajo de Tesis para optar por el título de \\ Doctor de la Universidad Nacional de La Plata en el área Física \\ Jesica María José Santillán
}

Director de Tesis: Dra. Lucía B. Scaffardi

Codirector de Tesis: Dr. Daniel C. Schinca

Lugar de trabajo: Centro de Investigaciones Ópticas

Febrero de 2013 


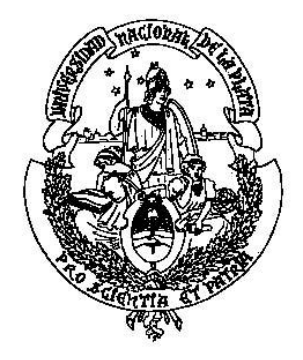

UNIVERSIDAD NACIONAL DE LA PLATA

FACULTAD DE CIENCIAS EXACTAS

DEPARTAMENTO DE FÍSICA

Estudio de las propiedades ópticas de materiales nanoestructurados y aplicaciones

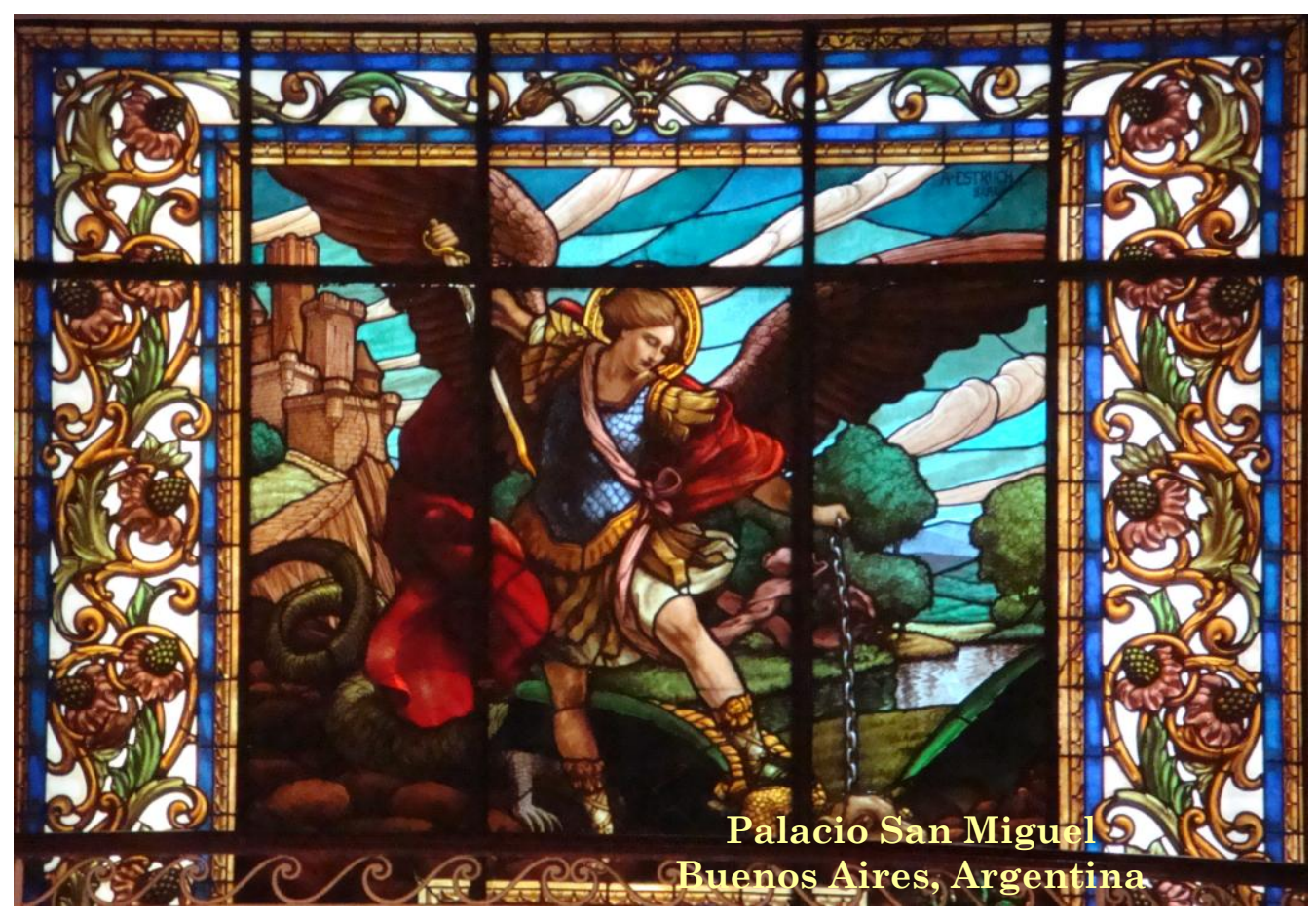

Tesis Doctoral

Jesica María José Santillán 
Con todo mi amor a mi madre Maemi 


\section{Índice general}

Resumen I

$\begin{array}{lll}\text { Abstract } & \text { V }\end{array}$

Prólogo IX

1 Introducción 1

2 Absorción y Scattering de partículas esféricas 11

2.1. Esferas simples 12

2.1.1. Teoría de Mie 13

2.1.2. Aproximación de Rayleigh 20

2.2. Esferas núcleo-recubrimiento $\quad 25$

3 Modelado de la función dieléctrica de metales 29

3.1. Ecuaciones de Maxwell y relaciones constitutivas 30

3.2. Función dieléctrica macroscópica para metales 32

3.2.1. Modelo de Lorentz 35

3.2.2. Contribución de los electrones libres 36

3.2.3. Contribución de los electrones ligados $\quad 39$

3.3. Función dieléctrica de partículas nanométricas

$(a<10 \mathrm{~nm}) \quad 42$

3.3.1. Corrección de los electrones libres por tamaño 42 
3.3.2. Inclusión de la corrección de electrones libres en la función dieléctrica

3.3.3. Contribución de los electrones ligados: transición única

3.3.4. Contribución de los electrones ligados: transiciones múltiples

3.3.4.1. Corrección de los electrones ligados por tamaño

4 Propagación de ondas electromagnéticas en interfases planas metal-dieléctrico

4.1. Polaritones plasmones superficiales en una interfase plana

4.1.1. Exitación del polaritón plasmón superficial 70

$\begin{array}{ll}\text { 4.2. Sistema de multicapas } & 71\end{array}$

4.3. Configuración de Kretschmann 74

5 Generación de nanopartículas metálicas por ablación láser. Análisis por técnicas espectroscópicas

5.1. Fabricación de nanopartículas por ablación láser de pulsos ultracortos

5.2. Espectroscopía de extinción óptica 84

5.3. Técnicas complementarias de análisis 88

6 Caracterización óptica de nanoestructuras metálicas esféricas

Nanopartículas esféricas de cobre y plata

6.1. Determinación de parámetros de electrones ligados de $\mathrm{Cu}$ y $\mathrm{Ag}$ en la función dieléctrica

6.1.1. Función dieléctrica de $\mathrm{Cu}$ y $\mathrm{Ag}$ dependiente del tamaño 
6.1.2. Análisis del comportamiento de los espectros de extinción

6.2. Determinación de tamaños y estructuras de partículas nanométricas y subnanométricas

6.2.1. Sobre la formación de óxido de cobre en nanopartículas

6.2.2. Influencia del shell de $\mathrm{Cu}_{2} \mathrm{O}$ en los espectros de extinción

6.2.3. Ajuste de los espectros de extinción de las suspensiones coloidales

\section{Aplicaciones al sensado}

7.1. Partícula aislada de plata-óxido de plata

7.1.1. Sensor de partícula aislada de Ag-óxido de Ag

7.1.2. Diseño y aplicación del método paramétrico

7.2. Películas nanométricas de $\mathrm{Ag}$ y $\mathrm{Ag}_{2} \mathrm{O}$

7.2.1. Diseño de un sensor de oxígeno basado en un dispositivo Kretschmann

7.2.2. Análisis de reflectividad para polarizaciones $p$ y $s$

7.2.3. Procedimiento híbrido de reflectancia SPPR-OWMC para la determinación de espesores de $\mathrm{Ag}_{2} \mathrm{O}$

8 Conclusiones 


\section{Resumen}

Durante los últimos años, ha adquirido importancia creciente el estudio de la interacción radiación visible-materia (fotónica), en la que la primera queda confinada a volúmenes de dimensiones mucho menores que la longitud de onda de la luz. El conocimiento de los procesos físicos que ocurren en dicha interacción permiten, entre otras cosas, el desarrollo de innumerables aplicaciones en diversos campos de la ciencia y la tecnología que abarcan desde la biomedicina hasta las telecomunicaciones.

La materia en escala nanométrica presenta propiedades ópticas, eléctricas, y magnéticas muy diferentes de las que posee en estado macroscópico. El estudio de las propiedades ópticas de estructuras nanométricas constituye un área de gran importancia no totalmente resuelta para el posible desarrollo de nuevos materiales y aplicaciones.

Esta Tesis combina un aspecto de desarrollo experimental y otro relacionado con el modelado teórico de las propiedades ópticas de nanoestructuras esféricas simples y núcleo-recubrimiento con el fin de complementar, reforzar y enriquecer la interpretación de los resultados obtenidos.

El desarrollo experimental está relacionado con la fabricación de partículas nanométricas y subnanométricas en medios líquidos por ablación 
láser de blancos sólidos de cobre y plata con pulsos ultracortos de Ti:Za, a diferentes energías y en distintos medios. Asimismo se obtienen espectros de extinción óptica y Raman a partir de las suspensiones coloidales generadas.

El aspecto teórico de esta Tesis abarca el modelado teórico de la función dieléctrica compleja de cobre y plata, y el estudio por separado del comportamiento de la contribución de los electrones libres y ligados considerando las modificaciones por tamaño para radios menores a $10 \mathrm{~nm}$. Teniendo en cuenta estas correcciones, se analizan los coeficientes de extinción de ambos metales para distintos tamaños de partículas nanométricas y subnanométricas y diferentes estructuras en función de la longitud de onda.

A partir del ajuste de los espectros de extinción experimentales de las suspensiones coloidales generadas para ambos metales nobles, se determina la composición, estructura, configuración y distribución de tamaños.

Por otra parte, se presentan aplicaciones orientadas al diseño de dos tipos de sensores plasmónicos de oxígeno: uno de ellos, basado en espectroscopía de extinción óptica de nanopartícula aislada de plata, y el otro de películas de plata de espesor nanométrico fundamentado en la resonancia del polaritón plasmón superficial en la configuración de Kretschmann. Para ambos casos, la presencia de oxígeno se determina a través de la medición del espesor de óxido de plata crecido sobre la nanoestructura de plata.

Finalmente, mediante el análisis de determinados parámetros característicos del espectro de extinción de las nanopartículas aisladas de plata, se establece un protocolo sencillo para conocer el tamaño del radio del núcleo y el espesor del recubrimiento de óxido. Para el caso del sensor de película delgada de plata, el estudio del comportamiento de parámetros característicos como el mínimo de la reflectividad, ancho total a altura media y reflectividad a determinados ángulos de la multicapa de plata-óxido 
de plata para polarizaciones de onda $p$ y $s$, permite establecer un protocolo de medición de reflectividad híbrido de Resonancia del Polaritón Plasmón Superficial - Acoplamiento del Modo de Guía de Onda Óptica para determinar y controlar in situ el espesor de la capa de óxido durante el proceso de crecimiento.

Palabras claves: plasmónica, nanopartículas, espectroscopía de extinción óptica, función dieléctrica, electrones libres y ligados, ablación láser, pulsos ultracortos, sensor de partícula aislada, sensor Kretschmann, películas nanométricas, resonancia plasmónica. 


\section{Abstract}

In recent years, it has become increasingly important the study the visible radiation-matter interaction field (photonics), wherein the former is confined to volume dimensions much smaller than the wavelength of light. Knowledge of the physical processes that occur in such interactions allow, among other things, the development of numerous applications in various fields of science and technology ranging from biomedicine to telecommunications.

Nanoscale material present optical, electrical, and magnetic properties very different from those observed in macroscopic state. The study of the optical properties of nanometric structures is an area of great importance not fully resolved for the possible development of new materials and applications.

This Thesis combines an experimental aspect and a theoretical modeling of the optical properties of simple and core-shell spherical nanostructures in order to complement, enhance and enrich the interpretation of the results.

Experimental development is related to the manufacture of nanometric and subnanometric particles in liquid media by laser ablation of solid copper and silver targets by ultrashort pulsed Ti:Sa laser, at different 
energies and different media. Likewise, optical extinction spectra and Raman spectra are obtained from the generated colloidal solutions.

The theoretical aspect of this Thesis covers theoretical modeling of complex dielectric function of copper and silver, and the separate study of the behavior of the contribution of free and bound electrons considering changes by size for radii smaller than $10 \mathrm{~nm}$. Given these corrections, an analysis of the extinction coefficients of both metals for different nanometric and subnanometric particle sizes proceeds.

From the fit of the extinction spectra of the experimentally generated colloidal suspensions for both noble metals, composition, structure, configuration and size distribution could be determined.

Applications are oriented to the design of two types of plasmonic oxygen sensors: one based on optical extinction spectroscopy of single silver nanoparticle and the other is based on surface plasmon polariton resonance of nanometric thickness silver films based on Kretschmann configuration. For both cases, the presence of oxygen is determined by measuring the thickness of oxide grown on the silver nanostructure.

Finally, by analyzing certain characteristic parameters of the extinction spectrum of single silver nanoparticles, a simple core radius shell thickness sizing protocol can be developed. In the case of thin-film silver sensor, studying the behavior of characteristic parameters such as minimum reflectivity, full width at half maximum and fixed-angle reflectivity of the multilayer silver-silver oxide $p$ and $s$ wave polarizations, protocol allows for a measurement of reflectivity hybrid Surface Plasmon Polariton Resonance - Coupling Mode Optical Waveguide to determine and monitor in situ the thickness of the oxide layer during the growth process. 
Keywords: plasmonics, nanoparticles, optical extinction spectroscopy, dielectric function, free and bound electrons, laser ablation, ultrashort laser pulses, single particle sensor, Kretschmann sensor, nanometric films, plasmonic resonance. 


\section{Prólogo}

La nanociencia es un área de investigación orientada al estudio de propiedades físicas y químicas de materiales con dimensiones inferiores a los $100 \mathrm{~nm}$. Dentro de esta gran área, la nanofotónica, definida como la fusión de la nanotecnología y la fotónica, emerge como un campo con nuevos desafíos tanto para la investigación básica como para desarrollos tecnológicos de frontera en química de síntesis, catálisis, nanofluidos, ciencia de materiales, superconductores de alta temperatura [Zuo et al. 1999, Wang et al. 2004, Larsen and Noriega 2004, Zhu et al. 2005, Patel et al. 2005, Novo et al. 2008], electrónica, integración fotónica en dispositivos miniaturizados para su uso en nanosensores biológicos y biomedicina [Xia et al. 2000, Prodan et al. 2003, Jiang et al. 2003, Bogatyrev et al. 2004, Haes et al. 2004, Elechiguerra et al. 2005, Moran et al. 2005, Dieringer et al. 2006, Zhao et al. 2006, Anker et al. 2008], entre otras.

La plasmónica, que forma parte de un área más extensa denominada nanofotónica, se caracteriza por el confinamiento de campos electromagnéticos en volúmenes inferiores a la longitud de onda como resultado de la interacción entre una onda electromagnética y los electrones de conducción en interfases metal-dieléctrico o en pequeñas nanoestructuras metálicas. 
En 1959 Richard Feynman (Conferencia "There's plenty of room at the bottom”, 29 de diciembre de 1959, Caltech), expresó por primera vez la posibilidad de la manipulación mecánica de átomos para realizar la síntesis de compuestos como alternativa a los métodos químicos tradicionales. Estas ideas dispararon el desarrollo de dispositivos de microscopía de muy alta resolución como el microscopio de fuerza atómica (AFM, Atomic Force Microscope) y el microscopio de efecto túnel (STM, Scanning Tunneling Microscope).

Si bien esta idea se manifestó con asidero científico a mediados del siglo XX, es conocido que ya desde la época del imperio romano y pasando por la Edad Media, algunos alquimistas y artesanos utilizaban pequeñas partículas metálicas en la manufactura de vidrio para crear obras de arte y adornos con características de coloración muy particulares, que hoy día se atribuyen a efectos de interacción radiación-materia en escala nanométrica.

La interacción de radiación electromagnética en la región UV-visible del espectro con nanoestructuras metálicas da origen a fenómenos ópticos característicos relacionados con la geometría de la nanoestructura, del tamaño y del medio circundante. En el caso de películas metálicas delgadas se generan ondas superficiales evanescentes acopladas a la oscilación de los electrones de conducción denominadas "polaritones plasmones superficiales". Estas ondas producen un refuerzo del campo eléctrico confinado a unas pocas decenas de nanómetros de la interfase, lo cual permite sensar cambios en las características del medio dieléctrico en dicho volumen. En el caso de nanopartículas metálicas, la oscilación acoplada de los electrones de conducción posee modos propios (llamados "plasmones") que se manifiestan como resonancias en el espectro de extinción de la solución coloidal de dichas partículas. Estas resonancias plasmónicas pueden utilizarse para determinar el tamaño y estructura de las nanopartículas, como así también, 
en el caso de una nanopartícula aislada, para el sensado de propiedades del medio circundante en las cercanías de la misma.

En esta Tesis se estudiarán, por un lado las propiedades ópticas de partículas esféricas nanométricas y subnanométricas (metálicas y de óxido metálico) de dos tipos de estructuras: simples y núcleo-recubrimiento (coreshell), y por el otro las propiedades ópticas de películas metálicas delgadas. Combina un aspecto de desarrollo experimental y otro relacionado con el modelado teórico para interpretar los resultados obtenidos, de manera de complementar y reforzar ambos enfoques.

A continuación se describe el esquema de la organización de esta Tesis.

En el Capítulo 1 se realizará una breve reseña de la plasmónica y se introducirán algunos conceptos necesarios para una clara interpretación de este trabajo.

En el Capítulo 2 se hará una revisión de los conceptos fundamentales de absorción y scattering de luz en partículas esféricas. En particular se describirá en detalle el fenómeno de extinción para dos tipos de estructuras esféricas tales como esferas simples y esferas núcleorecubrimiento, las cuales serán analizadas en esta Tesis.

El Capítulo 3 versará a cerca del modelado de la función dieléctrica compleja metálica y su corrección por tamaño para partículas nanométricas por debajo de $10 \mathrm{~nm}$ y subnanométricas $(<1 \mathrm{~nm})$. En particular se presentará en detalle la corrección por tamaño en las contribuciones de los electrones libres y ligados, y se estudiará su influencia en los espectros de extinción de dichas partículas. Este modelado de la función dieléctrica compleja se utilizará en los cálculos teóricos de los espectros de extinción. 
En el Capítulo 4 se abordará la fundamentación teórica de la propagación de ondas electromagnéticas en una o varias interfases planas, conceptos que serán utilizados en esta Tesis para el diseño y aplicación de sensores tipo Kretschmann, basados en el principio de resonancia plasmónica. Se presentarán algunas aplicaciones a modo de ejemplo y se señalará la importancia de este tipo de dispositivo.

El Capítulo 5 detallará el arreglo y el procedimiento experimental para la fabricación de las diferentes especies de partículas nanométricas mediante ablación láser de pulsos ultracortos de Ti:Za sobre un blanco sólido metálico de cobre o plata, en diferentes medios líquidos y a distintas energías.

Luego a través de espectroscopía de extinción óptica, se caracterizarán las suspensiones coloidales de cobre y plata generadas anteriormente. Esta técnica será complementada con HRTEM (High Resolution Transmission Electron Microscopy) y AFM. Además se utilizará espectroscopía Raman para la caracterización de los componentes de las estructuras simples o núcleo-recubrimiento presentes en las suspensiones coloidales.

En el Capítulo 6 se presentarán los resultados referidos a la caracterización óptica de suspensiones coloidales de partículas nanométricas. En primer lugar, a través del modelado de la función dieléctrica compleja, se determinarán los parámetros relacionados a la contribución de los electrones ligados para cobre y plata. Luego mediante el análisis y ajuste de los espectros de extinción experimentales, se caracterizará el tamaño, estructura y composición de las suspensiones coloidales obtenidas.

El Capítulo 7 describirá las aplicaciones al sensado de dos tipos de sensores plasmónicos, uno basado en espectroscopía de extinción de nanopartículas aisladas de plata y el otro fundamentado en la resonancia 
del polaritón plasmón superficial en la configuración de tipo Kretschmann. Se analizará la presencia de oxígeno a través de la medida de los espesores de películas nanométricas de óxido de plata crecidas sobre una base de plata.

Finalmente en el Capítulo 8 se expondrán las conclusiones generales de los trabajos desarrollados en esta Tesis y se describirán algunas líneas de investigación futuras como una continuación de este trabajo.

Además se enumerarán las publicaciones y comunicaciones a congresos producidas a partir de algunos de los aspectos originales de este trabajo de Tesis. 


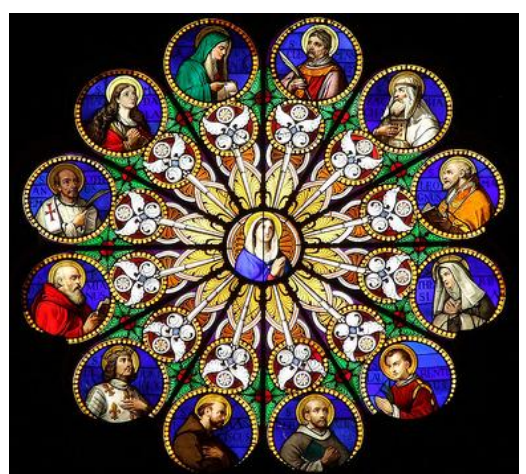

Santa Maria sopra Minerva Basílica de Roma, Italia

\section{Capítulo 1}

\section{Introducción}

La nanofotónica abarca las mismas temáticas básicas y aplicadas que la fotónica en la escala nanométrica. En cuanto a los aspectos básicos, se pueden mencionar las interacciones átomo-fotón en la región del campo cercano, absorción y emisión de luz en sistemas confinados y desarrollo de conceptos teóricos por medio de métodos basados en la función de Green diádica y de multipolos múltiples. Los aspectos aplicados comprenden, entre otros, nanolitografía por plasmones, microscopía óptica de superresolución, almacenamiento óptico de datos de alta densidad y microscopía multifotónica. Estos distintos campos se resumen en el esquema de la Figura 1.1.

La superación del límite de difracción por exploración del campo cercano de nanoestructuras metálicas iluminadas con cierta longitud de onda, ha dado lugar a numerosas investigaciones tanto sobre sus aspectos fundamentales como aplicados. Sin embargo, muchas de las aplicaciones prácticas al sensado se realizan a través de mediciones de transmitancia o absorbancia espectral en el campo lejano, incluyendo resonancias plasmónicas localizadas (partículas), en las que se utilizan dispositivos y detectores de acceso corriente en laboratorios de óptica. Todos los resultados 
de este trabajo de Tesis corresponden a mediciones realizadas en el campo lejano.
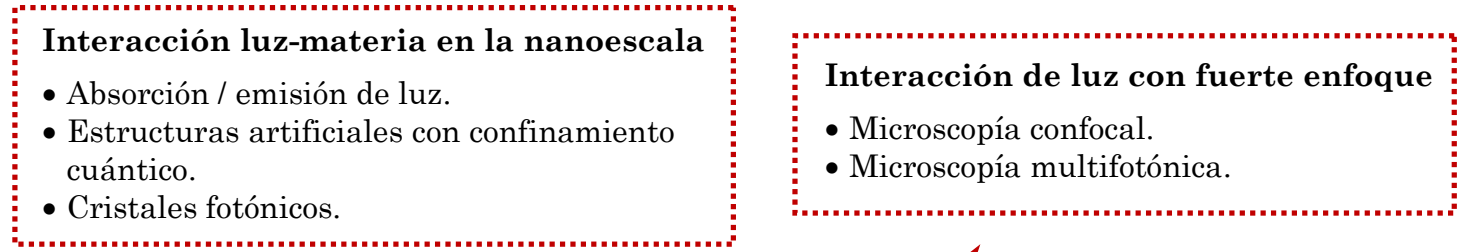

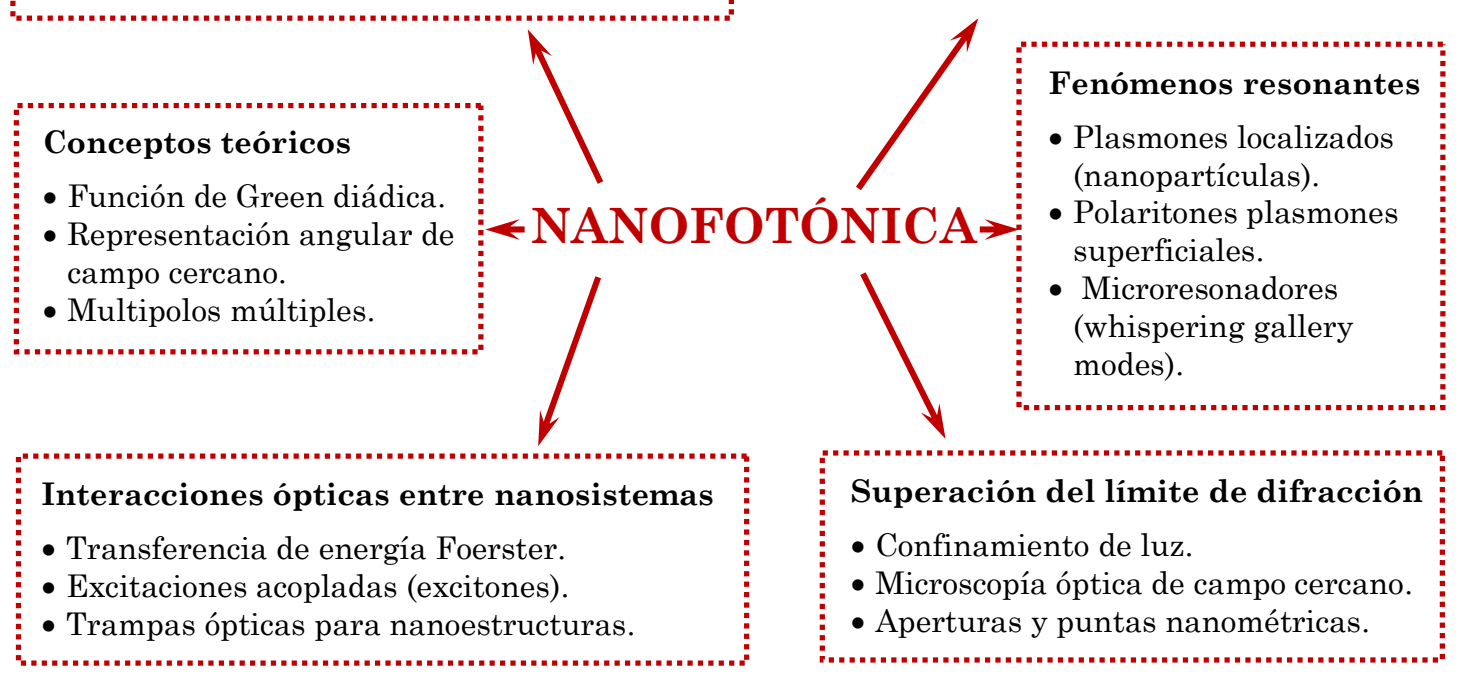

Figura 1.1: Áreas que integran la nanofotónica.

La plasmónica es un área de gran desarrollo dentro del campo de la nanofotónica, que explora la forma en que los campos electromagnéticos pueden ser confinados a dimensiones del orden o más pequeñas que la longitud de onda. Se basa en el estudio de los procesos de interacción entre la radiación electromagnética y los electrones de conducción en interfases metal-dieléctrico o en nanoestructuras metálicas, lo cual genera un refuerzo óptico de campo cercano para dimensiones sub-longitud de onda.

Los comportamientos que se observan como resultado de dicha interacción pueden describirse en base a la existencia de plasmones - 
oscilaciones colectivas del gas de electrones libres presentes en un metal tipo Drude - que en muchos casos suceden a frecuencias ópticas.

La descripción matemática de las ondas superficiales de oscilación de electrones (plasmones superficiales) fue desarrollada a comienzos del siglo $\mathrm{XX}$ en el contexto de ondas de radio que se propagaban a lo largo de la superficie de un conductor de conductividad finita [Sommerfeld 1899, Zenneck 1907].

La observación de una disminución anómala de la intensidad en el espectro generado cuando la luz visible se difractaba en una red de difracción metálica [Wood 1902], no fue conectada con los trabajos teóricos de Sommerfeld sino hasta mediados de siglo [Fano 1941]. Alrededor de estos años, se estudiaron fenómenos de pérdida de energía de electrones cuando un haz de éstos se difractaba en láminas delgadas metálicas [Ritchie 1957]. En 1968 se relacionaron estos resultados con el trabajo original del mismo autor sobre redes de difracción en el dominio óptico. La excitación de las ondas superficiales de Sommerfeld con luz visible usando un prisma de acoplamiento fue lograda en 1968 por Kretschmann y Raether unificando todos estos fenómenos con el modelo de "polaritones plasmones superficiales" [Kretschmann and Raether 1968].

Desde entonces los trabajos de investigación en este campo se concentraron en la región visible del espectro y llevaron a un redescubrimiento en este dominio de fenómenos observados en el campo de las microondas y los $\mathrm{THz}$ hacia principios del siglo XXI, cerrando así el círculo de los trabajos originales realizados 100 años atrás. La historia de plasmones (o plasmones superficiales localizados para algunos autores) en nanoestructuras metálicas es menos turbulenta, ya que las nanopartículas metálicas se usaron por primera vez en los tiempos del imperio romano para la coloración de vidrios. Una muy linda ilustración de esta aplicación 
histórica es la famosa copa de Lycurgus (siglo IV AC), que fue creada por antiguos artistas romanos y hoy se exhibe en el Museo Británico de Londres (Figura 1.1 (a)). Si esta copa es iluminada desde afuera con una fuente de luz blanca, las partículas de oro más grandes incrustadas en la misma dispersan predominantemente el verde haciéndola ver de ese mismo color. Sin embargo, cuando la copa se ilumina desde adentro las partículas más pequeñas absorben el verde y el azul, logrando de esta manera observarla de color rojo. Otra conocida aplicación es la coloración de ventanas de las catedrales en la Edad Media (Figura 1.1 (b)), donde algunos colores están determinados por la presencia de diferentes nanopartículas metálicas en el interior del vidrio. Dichos colores dependen del tamaño, forma y tipo de metal utilizado.

La descripción matemática de la respuesta electromagnética (scattering y absorción) de partículas esféricas a una onda incidente, fue desarrollada en 1908 por Gustav Mie, [Mie 1908]. El advenimiento de las modernas computadoras permitió manejar la complejidad de los cálculos de la solución de Mie, abriendo la posibilidad para la exploración de distintas configuraciones y tamaños en la nanoescala.
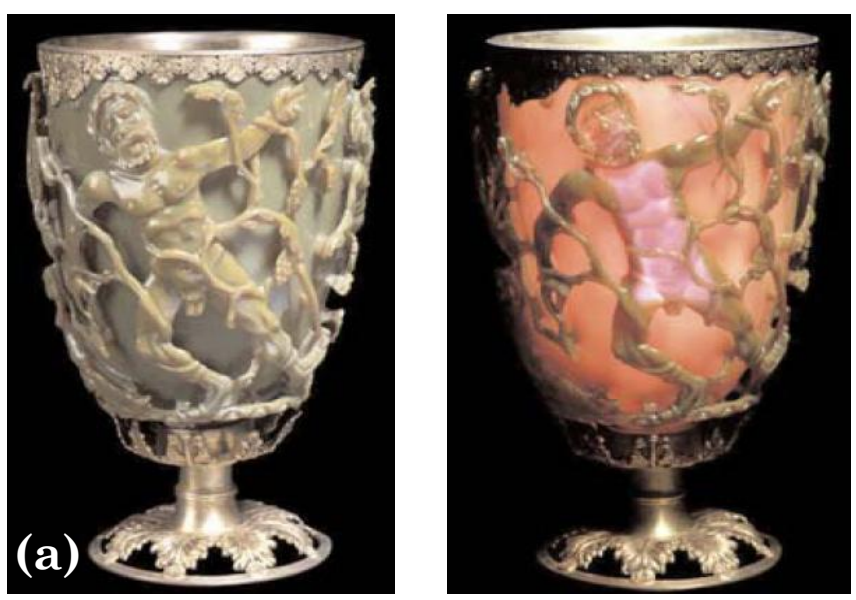


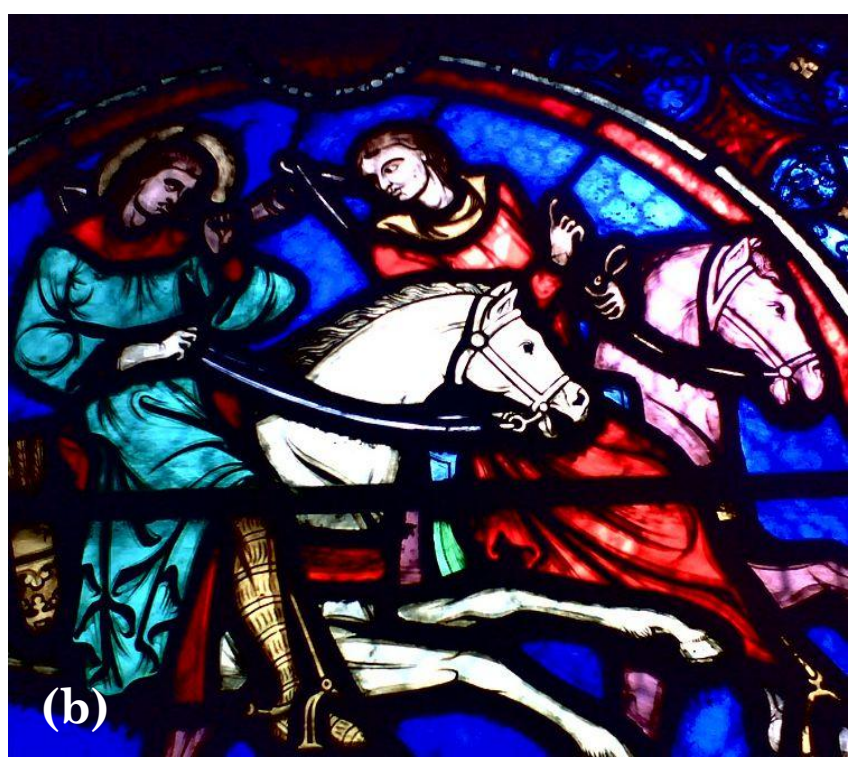

Figura 1.2: Nanotecnología en la antigüedad. (a) Copa de Lycurgus, del siglo IV AC, se exhibe en el Museo Británico de Londres, con diferente iluminación y, (b) detalle de un vitral de la Catedral de Notre Dame del período medieval.

En una primera aproximación de la materia, el modelo microscópico de Lorentz, trata al sistema electrón-ion como un oscilador armónico clásico, es decir, partículas ligadas a una posición de equilibrio por una fuerza restauradora.

En los metales, los electrones pertenecientes a las órbitas atómicas externas no están localizados (ligados), sino que se pueden considerar en una primera aproximación como libres. Este caso especial que considera a los electrones libres como un gas ideal, se conoce como el modelo de DrudeLorentz.

Cuando una onda electromagnética interactúa con los electrones de la banda de conducción en los metales, se produce la oscilación coherente de éstos denominada oscilación de plasma, donde la presencia de la distribución periódica de iones cargados positivamente está incluida en su masa efectiva [Ashcroft and Mermin 1976]. El campo electromagnético de 
una onda de luz incidente puede inducir la polarización de los electrones de conducción, lo que significa que éstos son desplazados con respecto a los iones positivos, hasta cancelar el campo dentro del metal. Cuando dicho campo deja de interactuar con el metal, los electrones continúan oscilando a una frecuencia llamada frecuencia de plasma $\left(\omega_{p}\right)$, hasta que la energía se pierde por disipación. La frecuencia de plasma de los electrones está definida por:

$$
\omega_{p}^{2}=\frac{n_{e} e^{2}}{m_{e} \varepsilon_{0}}
$$

donde $n_{e}$ es el número de electrones por unidad de volumen, $e$ es la carga del electrón, $m_{e}$ es la masa efectiva de los electrones libres y $\varepsilon_{0}$ es la permitividad del vacío. La mayoría de los metales tienen frecuencias de plasma en la región del ultravioleta, motivo por el cual son altamente reflectantes para longitudes de onda del visible. Cuando una onda de luz incide sobre un metal con una frecuencia que está por debajo de $\omega_{p}$ ésta se reflejará, porque los electrones apantallarán el campo eléctrico. Sin embargo, si la frecuencia está por encima de $\omega_{p}$ la onda se transmitirá por el metal, ya que los electrones no tienen una respuesta tan rápida como para apantallar el campo.

Si el gas de electrones está restringido en un cierto volumen de tamaño sub-longitud de onda, el desplazamiento total de los electrones con respecto a los iones positivos de la red da lugar a una fuerza restauradora que a su vez genera resonancias específicas de oscilación dependiendo de la geometría, del tamaño y del tipo de metal de la partícula, como así también del medio circundante, [Kelly et al. 2003]. 
Cuando un cuerpo metálico a escala nanométrica (típicamente por debajo de los $100 \mathrm{~nm}$ ) es irradiado, el campo electromagnético incidente genera una excitación colectiva de los electrones de conducción que oscilan coherentemente con el campo. Estos modos de oscilación llamados plasmones, cuya frecuencia cae para metales nobles en el rango visible del espectro, generan en las inmediaciones de la nanoestructura, una notable intensificación del campo incidente.

Dependiendo de la dimensión y de las condiciones de frontera del cuerpo metálico, los modos de oscilaciones plasmónicas se pueden clasificar en: plasmones volumétricos que existen en un cuerpo metálico grande 3D, plasmones superficiales generados en una interfase metal-dieléctrico, polaritones plasmones superficiales y plasmones de nanopartículas, muchas veces también llamados plasmones superficiales localizados según Klar [Klar 2007]. Para hacer una clara distinción entre estos modos de plasmones, en esta Tesis se utilizará la siguiente nomenclatura: "plasmones" a las oscilaciones plasmónicas de partículas nanométricas y "polaritones plasmones superficiales" a los que existen en una interfase plana metal-dieléctrico.

- Plasmón volumétrico: es una oscilación colectiva del gas de electrones libres dentro de la red cristalina del metal macroscópico, con una frecuencia característica que depende del tipo de metal. Las fluctuaciones en la densidad normal del gas de electrones se propagan como una fluctuación longitudinal de la densidad de carga.

- Plasmón de nanopartícula: es la excitación colectiva de los electrones de conducción en una nanopartícula metálica. Dado que el diámetro de una nanopartícula es del orden de la profundidad de penetración de las ondas electromagnéticas en los metales $(\sim 24 \mathrm{~nm}$ para la plata en el rango óptico [Homola et al. 1999]), la luz de 
excitación es capaz de penetrar la partícula. El campo dentro de la partícula desplaza los electrones de conducción colectivamente con respecto a la carga positiva fija de los iones de la red (Figura 1.3). Los electrones se acumulan en la superficie a un lado de la partícula. La atracción entre esta carga negativa y la carga positiva de los iones de la red restantes del lado opuesto, genera una fuerza restauradora. Si la frecuencia del campo de excitación está en resonancia con la frecuencia propia de esta oscilación colectiva, incluso un pequeño campo de excitación conduce a una fuerte oscilación.

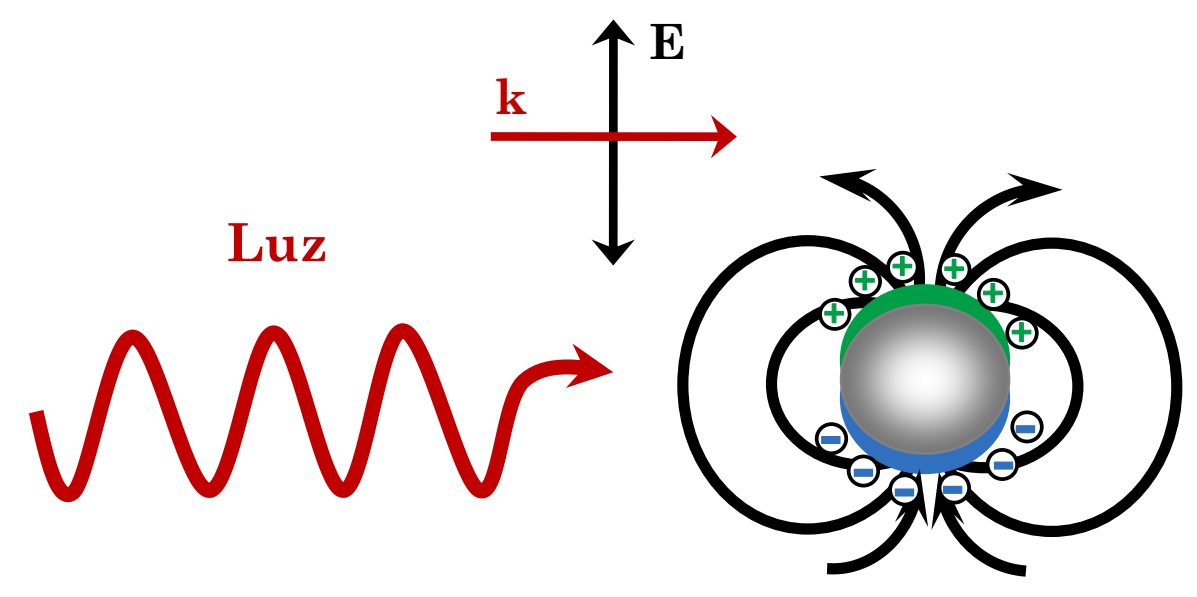

Figura 1.3: Oscilación plasmónica en una nanopartícula metálica, excitada por una onda de luz.

- Plasmón superficial: es la oscilación colectiva de la densidad de electrones cercanos a la superficie en una interfase metal-dieléctrico. Las oscilaciones de la densidad de carga asociadas a plasmones superficiales en la interfase dan lugar a un refuerzo de campo cercano que está espacialmente restringido a la vecindad inmediata de la superficie metálica (Figura 1.4 (a)). Como consecuencia de esto, el campo local en las vecindades de la superficie tiene una contribución 
adicional que lo hace varios órdenes de magnitud mayor que el campo incidente.

- Polaritón plasmón superficial: es el acoplamiento entre el modo de oscilación de la densidad de carga superficial y el campo electromagnético (plasmón superficial-fotón, Figura 1.4 (a)). En este caso, la onda superficial es propagante en el plano XY y evanescente en la dirección del eje Z, es decir, la intensidad del campo en el metal, así como en el dieléctrico, decae exponencialmente en la dirección normal a la superficie, mientras que en el plano de la interfase, la intensidad del campo y la distribución de carga en el metal se propagan como una onda longitudinal a lo largo de la superficie (Figura 1.4 (b)). La longitud de propagación del polaritón plasmón superficial para el caso de una interfase plata-aire es de $\sim 60 \mu \mathrm{m}$ a una longitud de onda de $633 \mathrm{~nm}$, mientras que la longitud de decaimiento de la onda evanescente en ambos medios es de $23 \mathrm{~nm}$ y $421 \mathrm{~nm}$, respectivamente [Novotny and Hecht 2007].

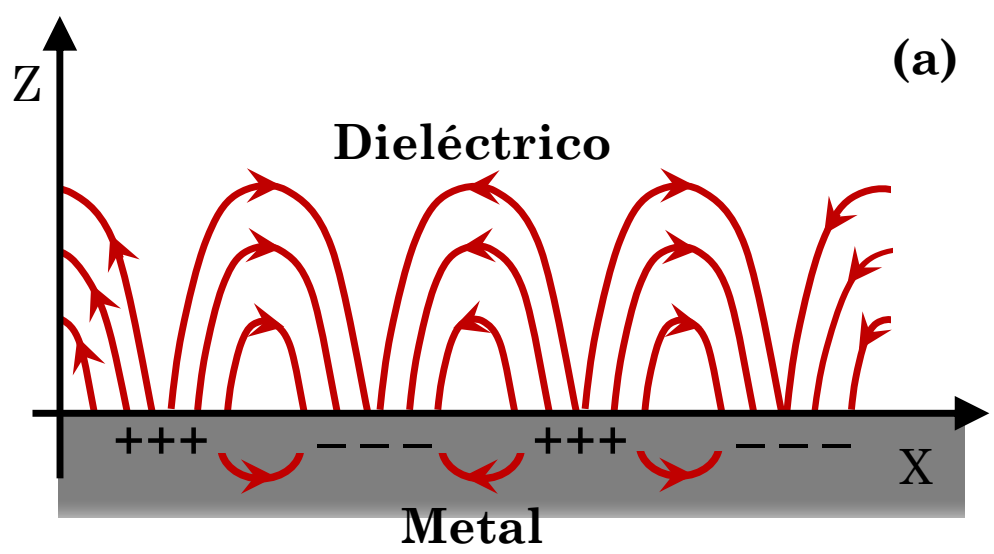




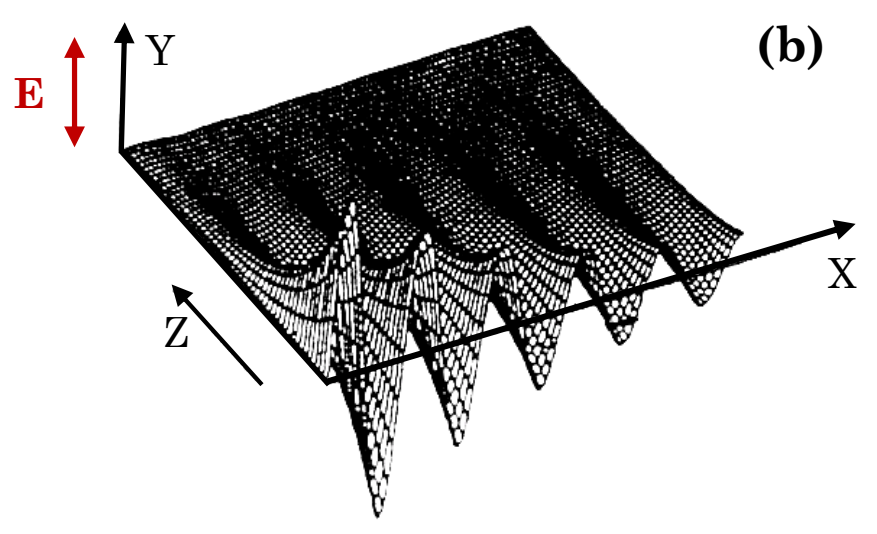

Figura 1.4: (a) Excitación de la densidad de carga electrónica en la superficie metálica (plasmón superficial), generada por una onda electromagnética y el campo eléctrico asociado (polaritón plasmón superficial) que se propagan a lo largo de la interfase metaldieléctrico. (b) Campo eléctrico evanescente asociado a los plasmones superficiales en una interfase metal-dieléctrico [Knoll 1998].

Finalmente, se menciona por completitud un aspecto interesante de la plasmónica, que se refiere al refuerzo en la intensidad transmitida por una abertura nanométrica en una película metálica con corrugaciones sublongitud de onda (transmisión extraordinaria) [Ebbesen et al. 1998, Thio et al. 2001]. En estos casos, una abertura circular de algunas centenas de nanómetros de diámetro realizada a través de una película de plata de espesor sub-longitud de onda y rodeada de anillos con estructura también sub-longitud de onda muestra un refuerzo de 10 veces la transmisión de la abertura aislada, debido al refuerzo de campo producido por la excitación de polaritones plasmones en la película metálica. 


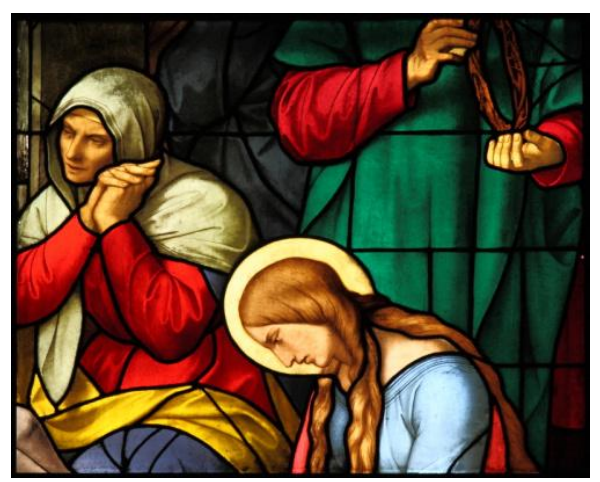

Catedral de Colonia

\section{Capítulo 2}

Colonia, Alemania

\section{Absorción y Scattering de partículas esféricas}

Cuando una partícula es iluminada por un haz de luz, la distribución angular de la intensidad de luz reirradiada como así también la cantidad de luz absorbida depende en forma detallada del tamaño, la forma y material de la partícula.

Dentro de este contexto, el problema con solución analítica más importante está relacionado al de una esfera de radio e índice de refracción arbitrarios. Aunque la solución formal a este problema ha estado disponible desde hace muchos años, sólo el advenimiento de las grandes computadoras de alto poder de cálculo ha permitido la resolución práctica de los complejos cálculos involucrados. En 1908, Gustav Mie desarrolló la teoría en un esfuerzo para comprender los variados colores en la absorción y scattering exhibidos por pequeñas partículas de oro suspendidas en agua [Mie 1908].

En este Capítulo se realiza una reseña del cálculo de la sección eficaz del scattering y la absorción en 3D de diferentes estructuras nanométricas tales como esfera simple y esfera núcleo-recubrimiento. Para los cálculos de 
las secciones transversales, se resumen los conceptos básicos relacionados con la teoría de Mie [Bohren and Huffman 1998] en el caso de partículas de tamaño e índice de refracción arbitrarios. Se analizan los modos electromagnéticos significativos dentro y fuera de una partícula cuando su tamaño es mucho menor que la longitud de onda incidente, considerando la aproximación cuasi-estática [Bohren and Huffman 1998, Maier 2007, Novotny and Hecht 2007]. Las expresiones encontradas serán utilizadas para la implementación de los programas de cálculo.

En esta Tesis se emplea la palabra "scattering" en lugar de la palabra en español "dispersión". En este contexto la palabra scattering se utiliza para hacer referencia a la redistribución de la luz cuando una onda electromagnética interactúa con un objeto. En óptica la palabra dispersión se refiere a la dependencia de la velocidad de propagación de una onda electromagnética en un material en función de su longitud de onda. Por esta razón, para evitar ambigüedades se ha decidido utilizar la palabra "scattering".

\subsection{Esferas simples}

En esta Sección se presenta, por un lado, el cálculo exacto del scattering de Mie para partículas esféricas simples de tamaño e índice de refracción arbitrarios, y por otra parte, la aproximación cuasi-estática de Rayleigh donde el tamaño de las partículas es mucho menor que la longitud de onda de la luz incidente. 


\subsubsection{Teoría de Mie}

La respuesta de una partícula a un campo electromagnético externo puede calcularse mediante la resolución de las ecuaciones de Maxwell. En un medio homogéneo e isótropo el campo electromagnético debe satisfacer la ecuación de onda dada por:

$$
\nabla^{2} \mathbf{E}+\mathrm{k}^{2} \mathbf{E}=0, \quad \nabla^{2} \mathbf{H}+\mathrm{k}^{2} \mathbf{H}=0
$$

donde $\mathrm{k}^{2}=\omega^{2} \varepsilon \mu$, siempre que se cumpla que la divergencia de estos campos sea nula,

$$
\nabla \cdot \mathbf{E}=0, \quad \nabla \cdot \mathbf{H}=0
$$

Además, E y $\mathbf{H}$ no son independientes, por lo tanto:

$$
\nabla \times \mathbf{E}=i \omega \mu \mathbf{H}, \quad \nabla \times \mathbf{H}=-i \omega \varepsilon \mathbf{E}
$$

Las soluciones que se proponen a estas ecuaciones son dos vectores $\mathbf{M}$ y $\mathbf{L}$ que tengan divergencia nula y estén vinculados por el rotor. Para satisfacer estas condiciones se define:

$$
\mathbf{M}=\nabla \times(\mathbf{c} \psi)
$$

como la divergencia del rotor de cualquier función vectorial es nula, usando identidades vectoriales se obtiene:

$$
\nabla^{2} \mathbf{M}+\mathrm{k}^{2} \mathbf{M}=\nabla \times\left\lfloor\mathbf{c}\left(\nabla^{2} \psi+\mathrm{k}^{2} \psi\right)\right\rfloor
$$


Entonces, M satisface la ecuación de onda vectorial, si $\psi$ es una solución de la ecuación de onda escalar

$$
\left(\nabla^{2} \psi+\mathrm{k}^{2} \psi\right)=0
$$

Para vincular el rotor de $\mathbf{M}$, se define otra función vectorial dada por:

$$
\mathbf{L}=\frac{\nabla \times \mathbf{M}}{\mathrm{k}}
$$

con divergencia nula, la cual también satisface la ecuación de onda vectorial

$$
\nabla^{2} \mathbf{L}+\mathrm{k}^{2} \mathbf{L}=0
$$

Por lo tanto, $\mathbf{M}$ y $\mathbf{L}$ tienen todas las propiedades requeridas de un campo electromagnético, de manera tal que el problema de encontrar soluciones a las ecuaciones de campo se reduce al problema relativamente sencillo de encontrar soluciones a la ecuación de onda escalar. La función escalar $\psi$ es denominada función generadora de los vectores armónicos $\mathbf{M}$ y $\mathbf{L}$ y el vector c es denominado vector piloto.

Como la simetría es esférica se puede tomar $\mathbf{c}=\mathbf{r}$ y se reemplaza en la ecuación (2.4). En consecuencia $\mathbf{M}$ es tangencial a la esfera de radio $a$ (Figura 2.1).

Por otra parte, $\mathbf{M}$ es una solución de la ecuación de onda vectorial en coordenadas polares esféricas. Realizando las sustituciones correspondientes en la ecuación de onda escalar en coordenadas esféricas, se obtienen tres expresiones independientes: 


$$
\begin{gathered}
\frac{d^{2} \Phi}{d \phi^{2}}+m^{2} \Phi=0 \\
\frac{1}{\operatorname{sen} \theta} \frac{d}{d \theta}\left(\operatorname{sen} \theta \frac{d \Theta}{d \theta}\right)+\left[n(n+1)-\frac{m^{2}}{\operatorname{sen}^{2} \theta}\right] \Theta=0 \\
\frac{d}{d r}\left(r^{2} \frac{d R}{d r}\right)+\left[\mathrm{k}^{2} r^{2}-n(n+1)\right] R=0
\end{gathered}
$$

donde las constantes de separación $m$ y $n$ están determinadas por las condiciones que debe cumplir $\psi$.

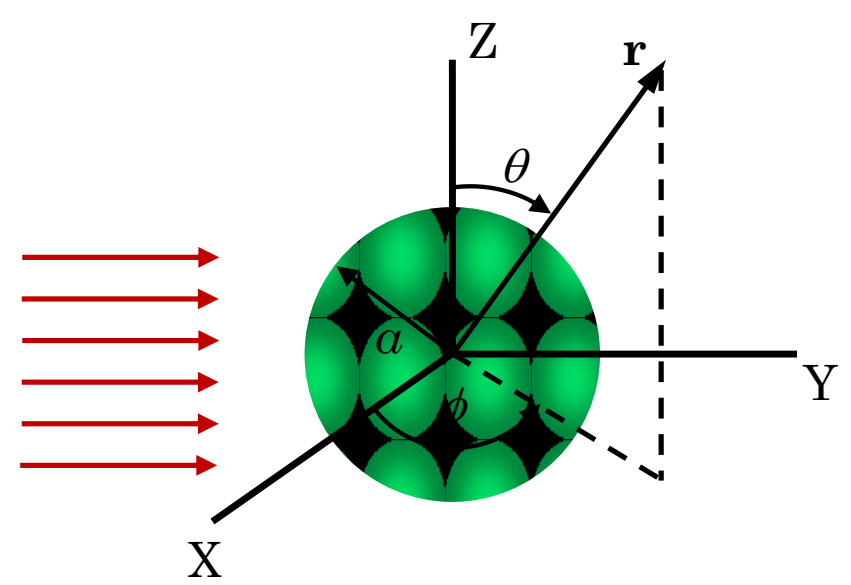

Figura 2.1: Sistema de coordenadas polares esféricas centrado en una partícula esférica de radio $a$.

La ecuación (2.9) se satisface para $m$ par ( $p$ ) e impar (o), entonces las soluciones linealmente independientes son:

$$
\Phi_{p}=\cos m \phi, \quad \Phi_{o}=\operatorname{sen} m \phi
$$


La función $\psi$ debe ser univaluada excepto en los límites entre medios con diferentes propiedades, pero en este caso se consideran las soluciones de la ecuación de onda escalar en el interior de la esfera, por lo tanto $m$ debe ser un entero positivo.

Las soluciones a la ecuación (2.10) se cumplen para valores finitos de $\theta=0$ y $\theta=\pi$. Estas funciones están asociadas a los polinomios de Legendre de primera clase de grado $n$ y orden $m$, donde $n=m, m+1, \ldots$. Estas funciones son ortogonales

$$
\int_{-1}^{1} P_{n}^{m}(\beta) P_{n^{\prime}}^{m}(\beta) d \beta=\delta_{n^{\prime} n} \frac{2}{2 n+1} \frac{(n+m) !}{(n-m) !}
$$

donde $\beta=\cos \theta, \delta_{n^{\prime} n}$ es la delta de Kronecker y $P_{n}$ son los polinomios de Legendre.

Si se introduce la variable adimensional $\rho=\mathrm{k} r$ y se define la función $Z=R \sqrt{\rho}$, y luego se reemplaza esta función en la ecuación (2.11), se tiene como soluciones linealmente independientes las funciones de Bessel de primera y segunda clase $J_{v}$ y $Y_{v}$, donde el orden es $v=n+1 / 2$. En consecuencia, las soluciones son funciones esféricas de Bessel dadas por:

$$
\begin{aligned}
& j_{n}(\rho)=\sqrt{\frac{\pi}{2 \rho}} J_{n+1 / 2}(\rho) \\
& y_{n}(\rho)=\sqrt{\frac{\pi}{2 \rho}} Y_{n+1 / 2}(\rho)
\end{aligned}
$$

Cualquier combinación lineal de estas funciones esféricas de Bessel es solución de la ecuación (2.11) y se denominan funciones esféricas de Hankel: 


$$
\begin{aligned}
& h_{n}^{(1)}(\rho)=j_{n}(\rho)+i y_{n}(\rho) \\
& h_{n}^{(2)}(\rho)=j_{n}(\rho)-i y_{n}(\rho)
\end{aligned}
$$

Las funciones generadoras que satisfacen la ecuación de onda escalar en coordenadas polares esféricas son:

$$
\begin{aligned}
& \psi_{p m n}=\cos m \phi P_{n}^{m}(\cos \theta) z_{n}(\mathrm{k} r) \\
& \psi_{i m n}=\operatorname{sen} m \phi P_{n}^{m}(\cos \theta) z_{n}(\mathrm{k} r)
\end{aligned}
$$

donde $z_{n}$ es cualquiera de las funciones esféricas de Bessel. Por otra parte, debido a la completitud de las funciones $\cos m \phi, \operatorname{sen} m \phi, P_{n}^{m}(\cos \theta)$ y $z_{n}(\mathrm{k} r)$, cualquier función que satisfaga la ecuación de onda escalar en coordenadas polares esféricas puede ser expandida en una serie de las funciones (2.17). Las expresiones de los armónicos esféricos generados por estas funciones son:

$$
\begin{array}{ll}
\mathbf{M}_{p m n}=\nabla \times\left(\mathbf{r} \psi_{p m n}\right), & \mathbf{M}_{o m n}=\nabla \times\left(\mathbf{r} \psi_{o m n}\right) \\
\mathbf{L}_{p m n}=\frac{\nabla \times \mathbf{M}_{p m n}}{\mathrm{k}}, & \mathbf{L}_{o m n}=\frac{\nabla \times \mathbf{M}_{o m n}}{\mathrm{k}}
\end{array}
$$

Una onda plana incidente polarizada en la dirección X, en una esfera arbitraria se escribe como:

$$
\mathbf{E}_{i}=E_{0} e^{i \mathrm{k} r \cos \theta} \hat{\mathbf{e}}_{x}
$$

El primer paso hacia la solución de este problema es expandir la expresión de esta onda plana en vectores armónicos esféricos. Por lo tanto, $\mathbf{E}$ y $\mathbf{H}$ se 
pueden expresar en forma expandida para el campo incidente $\left(\mathbf{E}_{i}, \mathbf{H}_{i}\right)$ y el dispersado $\left(\mathbf{E}_{s}, \mathbf{H}_{s}\right)$ por una esfera, entonces $\mathbf{E}_{i}$ y $\mathbf{H}_{i}$ toman la forma:

$$
\begin{aligned}
& \mathbf{E}_{i}=E_{0} \sum_{n=1}^{\infty} i^{n} \frac{2 n+1}{n(n+1)}\left(\mathbf{M}_{o 1 n}^{(1)}-i \mathbf{L}_{p 1 n}^{(1)}\right) \\
& \mathbf{H}_{i}=\frac{-\mathbf{k}}{\omega \mu} E_{0} \sum_{n=1}^{\infty} i^{n} \frac{2 n+1}{n(n+1)}\left(\mathbf{M}_{o 1 n}^{(1)}-i \mathbf{L}_{p 1 n}^{(1)}\right)
\end{aligned}
$$

con $\mathbf{E}_{s}$ y $\mathbf{H}_{s}$ dados por:

$$
\begin{aligned}
& \mathbf{E}_{s}=\sum_{n=1}^{\infty} E_{n}\left(i a_{n} \mathbf{L}_{p 1 n}^{(3)}-b_{n} \mathbf{M}_{o 1 n}^{(3)}\right) \\
& \mathbf{H}_{s}=\frac{\mathrm{k}}{\omega \mu} \sum_{n=1}^{\infty} E_{n}\left(i b_{n} \mathbf{L}_{o 1 n}^{(3)}+a_{n} \mathbf{M}_{p 1 n}^{(3)}\right)
\end{aligned}
$$

donde $E_{n}=i^{n} E_{0}(2 n+1) / n(n+1), \quad a_{n} \quad \mathrm{y} \quad b_{n}$ son los coeficientes de scattering expresados como:

$$
\begin{aligned}
& a_{n}=\frac{\mu \mathrm{m}^{2} j_{n}(\mathrm{~m} x)\left[x j_{n}(x)\right]^{\prime}-\mu_{1} j_{n}(x)\left[\mathrm{m} x j_{n}(\mathrm{~m} x)\right]^{\prime}}{\mu \mathrm{m}^{2} j_{n}(\mathrm{~m} x)\left[x h_{n}^{(1)}(x)\right]^{\prime}-\mu_{1} h_{n}^{(1)}(x)\left[\mathrm{m} x j_{n}(\mathrm{~m} x)\right]^{\prime}} \\
& b_{n}=\frac{\mu_{1} j_{n}(\mathrm{~m} x)\left[x j_{n}(x)\right]^{\prime}-\mu j_{n}(x)\left[\mathrm{m} x j_{n}(\mathrm{~m} x)\right]^{\prime}}{\mu_{1} j_{n}(\mathrm{~m} x)\left[x h_{n}^{(1)}(x)\right]^{\prime}-\mu h_{n}^{(1)}(x)\left[\mathrm{m} x j_{n}(\mathrm{~m} x)\right]^{\prime}}
\end{aligned}
$$

siendo $\mu$ у $\mu_{1}$ las permeabilidades magnéticas del medio circundante y de la partícula esférica respectivamente, $x=2 \pi N a / \lambda$ es el llamado parámetro de tamaño y $\mathrm{m}=N_{1} / N$ es el índice de refracción relativo, donde $N$ y $N_{1}$ son los índices de refracción del medio y de la partícula, 
respectivamente. Estos coeficientes de scattering dependen tanto del tamaño como de los parámetros constitutivos del material de la partícula.

En consecuencia, la sección eficaz de scattering de una esfera de radio arbitrario a está dada por:

$$
C_{s c a}=\frac{2 \pi}{\mathrm{k}^{2}} \sum_{n=1}^{\infty}(2 n+1)\left(\left|a_{n}\right|^{2}+\left|b_{n}\right|^{2}\right)
$$
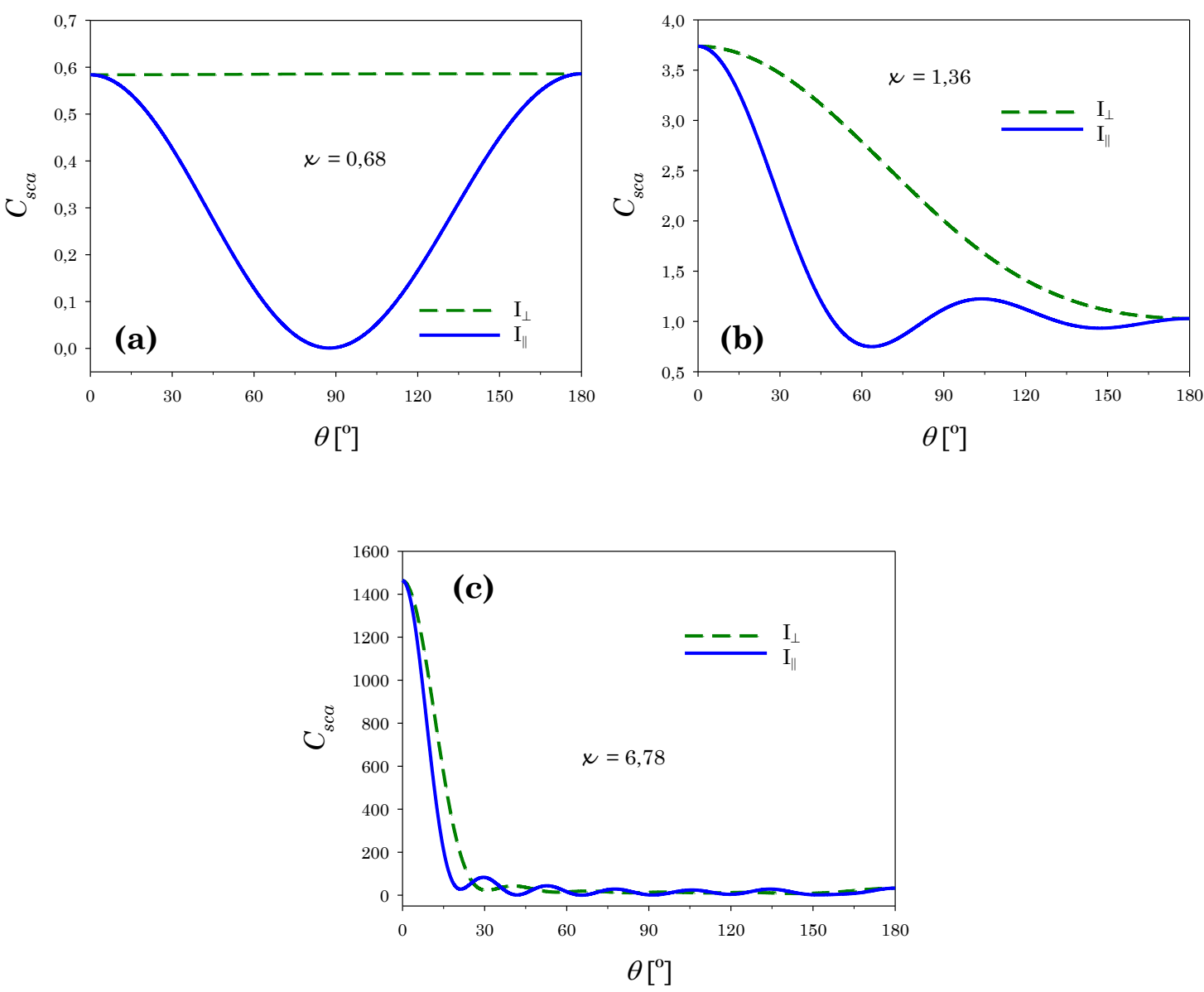

Figura 2.2: Scattering angular de partículas esféricas de oro inmersas en agua para polarizaciones paralela ( || ) y perpendicular $(\perp)$ y diferentes radios: (a) $50 \mathrm{~nm}$, (b) $100 \mathrm{~nm}$ y (c) $500 \mathrm{~nm}$. La longitud de onda incidente utilizada para el cálculo es $616,9 \mathrm{~nm}$ y los índices de refracción para la partícula y el medio a esta longitud de onda son: $N_{1}=0,21+i 3,272$ ([Johnson and Christy 1972]) y $N=1,332$, respectivamente. 
Las Figuras 2.2 (a), (b) y (c) muestran el comportamiento angular de la sección eficaz de scattering correspondiente a una esfera de oro inmersa en agua, calculada con la ecuación (2.23) considerando $\lambda=616,9 \mathrm{~nm}$ para tres parámetros de tamaño $x$ diferentes y dos estados de polarización de la luz incidente.

\subsubsection{Aproximación de Rayleigh}

La interacción de un campo electromagnético monocromático con una partícula mucho más pequeña que la longitud de onda del mismo, puede analizarse usando la simple aproximación cuasi-estática, ignorando los efectos de retardo. Esto asume que todos los puntos de una partícula responden simultáneamente al campo incidente.

Para una esfera pequeña con $a<<\lambda$, la representación como un dipolo ideal es válida en el régimen cuasi-estático. Por lo tanto, el campo eléctrico de un dipolo oscilante aproximado a la zona de campo cercano está dado por:

$$
\mathbf{E}(\mathbf{r}, t)=\frac{1}{4 \pi \varepsilon_{0}}[3 \mathbf{r}(\mathbf{r} \cdot \mathbf{p})-\mathbf{p}] \frac{e^{i \omega t}}{r^{3}}
$$

donde $\mathbf{p}$ es el momento dipolar y $\mathbf{r}$ es el vector unidad en la dirección del punto de interés. En el límite cuasi-estático, el campo eléctrico se puede representar por un potencial como $\mathbf{E}=-\nabla \Phi$, donde este potencial debe satisfacer la ecuación de Laplace $\nabla^{2} \Phi=0$ y las condiciones de frontera entre los medios adyacentes. Las soluciones de la ecuación de Laplace en coordenadas esféricas son de la forma: 


$$
\Phi(r, \theta, \varphi)=\sum_{n, m} b_{n, m} \Phi_{n, m}(r, \theta, \varphi)
$$

Aquí, los coeficientes constantes $b_{n, m}$ determinan las condiciones de frontera y $\operatorname{los} \Phi_{n, m}$ se expresan como:

$$
\Phi_{n, m}=\left\{\begin{array}{c}
r^{n} \\
r^{-n-1}
\end{array}\right\}\left\{\begin{array}{c}
P_{n}^{m}(\cos \theta) \\
Q_{n}^{m}(\cos \theta)
\end{array}\right\}\left\{\begin{array}{c}
e^{i m \varphi} \\
e^{-i m \varphi}
\end{array}\right\}
$$

donde $P_{n}^{m}(\cos \theta)$ son las funciones asociadas de Legendre y $Q_{n}^{m}(\cos \theta)$ son las funciones de Legendre de segunda clase. Proponiendo la continuidad de las componentes tangenciales del campo eléctrico y de las normales del desplazamiento eléctrico en la superficie de la esfera, y considerando que el campo eléctrico incidente es homogéneo y se encuentra en la dirección del eje X como se observa en la Figura 2.3, se pueden obtener expresiones para el potencial dentro $\left(\Phi_{1}\right)$ y fuera $\left(\Phi_{2}\right)$ de la esfera [Novotny and Hecht 2007]:

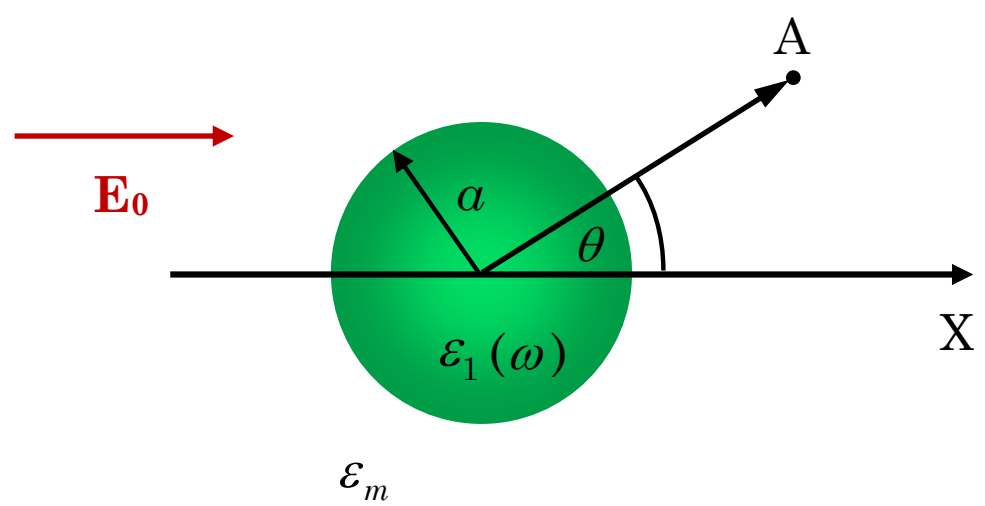

Figura 2.3: Esquema de una esfera homogénea situada en un campo electrostático $\mathbf{E}_{0}$ paralelo a la dirección de propagación en el eje X. 


$$
\begin{gathered}
\Phi_{1}=-E_{0} \frac{3 \varepsilon_{m}}{\varepsilon_{1}+2 \varepsilon_{m}} r \cos \theta \\
\Phi_{2}=-E_{0} r \cos \theta+E_{0} \frac{\varepsilon_{1}-\varepsilon_{m}}{\varepsilon_{1}+2 \varepsilon_{m}} \frac{a^{3} \cos \theta}{r^{2}}
\end{gathered}
$$

donde $\Phi_{2}$ es la suma de los potenciales incidente y dispersado, $\varepsilon_{1}$ y $\varepsilon_{m}$ son las constantes dieléctricas de la partícula y del medio circundante, respectivamente. Observando las expresiones (2.27) se puede notar que la condición de resonancia para una partícula esférica pequeña, se obtiene cuando $\varepsilon_{1}=-2 \varepsilon_{m}$. La distribución del campo eléctrico $\mathbf{E}=-\nabla \Phi$ se puede evaluar de los potenciales (2.27) obteniéndose:

$$
\begin{gathered}
\mathbf{E}_{1}=E_{0} \frac{3 \varepsilon_{m}}{\varepsilon_{1}+2 \varepsilon_{m}} \mathbf{n}_{x} \\
\mathbf{E}_{2}=E_{0} \mathbf{n}_{x}+E_{0} \frac{\varepsilon_{1}-\varepsilon_{m}}{\varepsilon_{1}+2 \varepsilon_{m}} \frac{a^{3}}{r^{3}}\left(2 \cos \theta \mathbf{n}_{r}+\operatorname{sen} \theta \mathbf{n}_{\theta}\right)
\end{gathered}
$$

Una característica importante del campo eléctrico es su homogeneidad dentro de la partícula. Por otra parte, si se compara la ecuación (2.24) con el campo dispersado (segundo término de la expresión (2.29)), se observa que es idéntico al campo electrostático de un dipolo situado en el centro de la esfera. Entonces el campo aplicado $\mathbf{E}_{0}$ induce un momento dipolar dentro de la esfera de magnitud proporcional a $\left|\mathbf{E}_{0}\right|$, siendo

$$
\mathbf{p}=\varepsilon_{0} \alpha \mathbf{E}_{0}
$$

donde $\alpha$ es la polarizabilidad definida como: 


$$
\alpha=4 \pi a^{3} \frac{\varepsilon_{1}-\varepsilon_{m}}{\varepsilon_{1}+2 \varepsilon_{m}}
$$

En consecuencia, la sección eficaz de scattering de una esfera simple se puede obtener dividiendo la potencia total irradiada del dipolo en la esfera por la intensidad de la onda plana incidente, dando como resultado:

$$
C_{s c a}=\frac{\mathrm{k}^{4}}{6 \pi}|\alpha|^{2}=\frac{8}{3} \pi \mathrm{k}^{4} a^{6}\left|\frac{\varepsilon_{1}-\varepsilon_{m}}{\varepsilon_{1}+2 \varepsilon_{m}}\right|^{2}
$$

siendo $\mathrm{k}$ el vector de onda en el medio circundante.

La potencia eliminada del haz incidente debido a la presencia de una partícula no solo se debe al fenómeno de scattering sino también a un fenómeno conocido como absorción. Por lo tanto, si se calcula la potencia disipada dentro de la partícula, se obtiene una expresión para la sección eficaz de absorción, dada por:

$$
C_{a b s}=\mathrm{k} \operatorname{Im}[\alpha]=4 \pi \mathrm{k} a^{3} \operatorname{Im}\left[\frac{\varepsilon_{1}-\varepsilon_{m}}{\varepsilon_{1}+2 \varepsilon_{m}}\right]
$$

La suma del fenómeno de scattering y el de absorción dan como resultado un fenómeno conocido como extinción, $C_{e x t}=C_{a b s}+C_{s c a}$. Las ecuaciones (2.32) y (2.33) demuestran que cuando una partícula pequeña con $a<<\lambda$ interactúa con un campo electromagnético, la eficiencia de absorción que es proporcional a $a^{3}$, domina sobre la eficiencia de sacttering, siendo ésta proporcional a $a^{6}$. Además se observa que, para el caso de nanopartículas metálicas tanto la absorción como el scattering están reforzados resonantemente cuando se cumple la condición 
$\operatorname{Re}\left[\varepsilon_{1}(\omega)\right]=-2 \varepsilon_{m}$, siendo ésta la resonancia del plasmón que se conoce como condición de Fröhlich.

En la Figura 2.4 se muestra el coeficiente de extinción $\left(Q_{e x t}=C_{e x t} / 2 \pi a^{2}\right)$ de una partícula esférica de oro en la aproximación cuasiestática para diferentes medios calculada con la expresión (2.33). Se observa que la resonancia del plasmón para el caso del aire se encuentra en $\lambda_{F, \text { aire }}=505 \mathrm{~nm}$, mientras que para el agua aparece en $\lambda_{F, \text { agua }}=520 \mathrm{~nm}$.

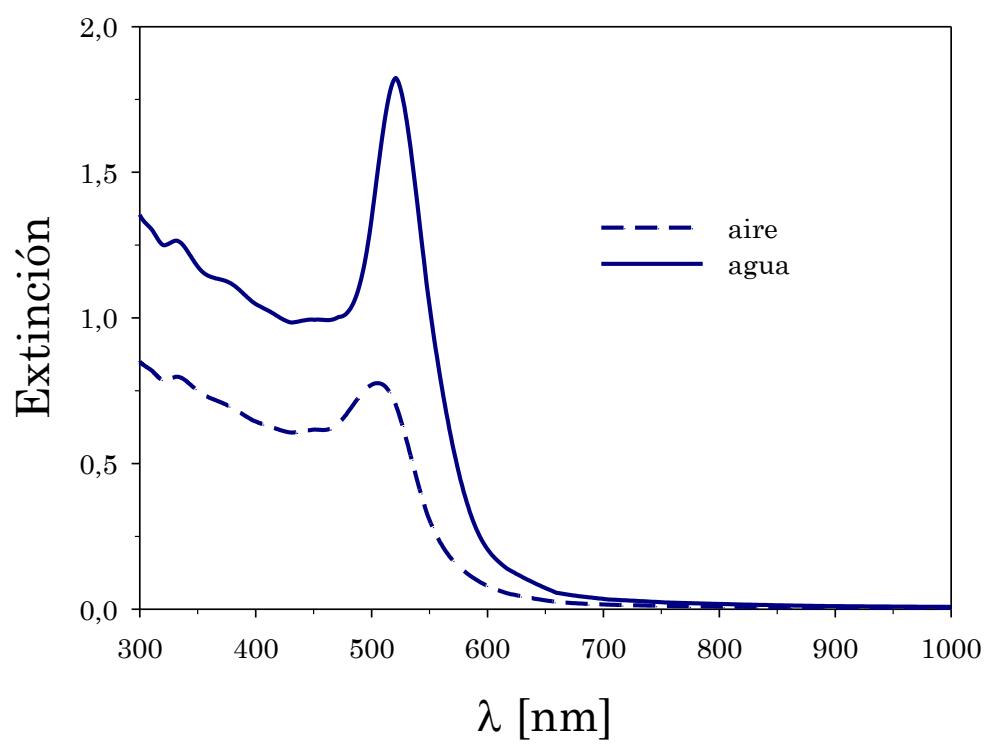

Figura 2.4: Coeficiente de extinción calculado para una esfera de oro de $20 \mathrm{~nm}$ de radio en aire y agua. Los datos de la función dieléctrica del oro fueron tomados de Johnson y Christy [Johnson and Christy 1972].

Considerando que la función dieléctrica para el aire es menor que la correspondiente al agua, es previsible que la longitud de onda de la resonancia del plasmón para el primero se produzca a una longitud de onda menor que para el segundo. 


\subsection{Esferas núcleo-recubrimiento}

Para el caso de estructuras más complejas, como esferas núcleorecubrimiento, la expresión de la polarizabilidad $\alpha$ es [Bohren and Huffman 1998]:

$$
\alpha=4 \pi a_{2}^{3} \frac{\left(\varepsilon_{2}-\varepsilon_{m}\right)\left(\varepsilon_{1}+2 \varepsilon_{2}\right)+f\left(\varepsilon_{1}-\varepsilon_{2}\right)\left(\varepsilon_{m}+2 \varepsilon_{2}\right)}{\left(\varepsilon_{2}+2 \varepsilon_{m}\right)\left(\varepsilon_{1}+2 \varepsilon_{2}\right)+f\left(2 \varepsilon_{2}+2 \varepsilon_{m}\right)\left(\varepsilon_{1}-\varepsilon_{2}\right)}
$$

donde $f=\left(a_{1} / a_{2}\right)^{3}$ es el cociente entre los volúmenes de los radios interno y externo de la estructura esférica, siendo $a_{1}=a_{\text {núcleo }}$ y $a_{2}=a_{\text {núcleo +recubrimiento }} ; \varepsilon_{1}$,

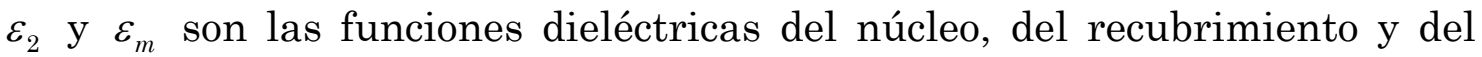
medio circundante, respectivamente (Figura 2.5).

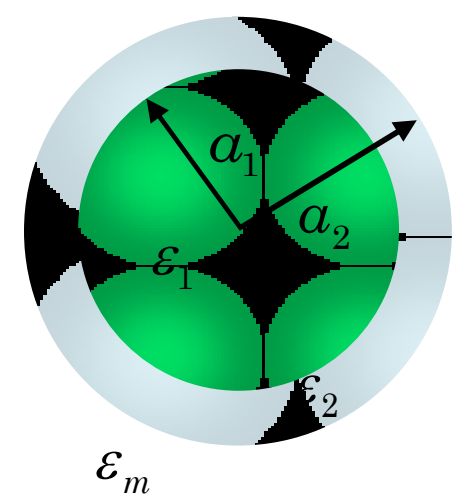

Figura 2.5: Representación esquemática de una esfera de estructura núcleorecubrimiento, donde $a_{1}$ es el radio del núcleo, $a_{2}$ es el radio de núcleo + recubrimiento; $\varepsilon_{1}, \varepsilon_{2}$ y $\varepsilon_{m}$ son las funciones dieléctricas del núcleo, del recubrimiento y del medio que rodea la partícula, respectivamente.

En la Figura 2.6 se muestran, a modo de ejemplo, los espectros de extinción de una nanopartícula de dióxido de silicio $\left(\mathrm{SiO}_{2}\right)$ de $10 \mathrm{~nm}$ de radio 
de núcleo para tres espesores diferentes de recubrimiento de oro inmersa en agua, calculados con las ecuaciones (2.32) y (2.33) donde la polarizabilidad utilizada corresponde a la expresión (2.34). Se observa un desplazamiento hacia longitudes de onda más largas a medida que disminuye el espesor de oro. Esta propiedad de sintonía de la longitud de onda de los plasmones es la base del diseño de dispositivos ópticos en la nanoescala para una gran variedad de aplicaciones [Shipway et al. 2000, Li et al. 2003]. En el área biológica, las energías de resonancia plasmónica de nanorecubrimientos (nanoshells) han sido ajustadas a la región del infrarrojo cercano por sus interesantes aplicaciones biomédicas. Por ejemplo, para el caso de tratamientos de tumores con nanopartículas, éstos pueden ser destruidos a través del calentamiento producido por la absorción de las mismas en el IR cercano [Hirsch et al. 2003a, Prodan et al. 2003].

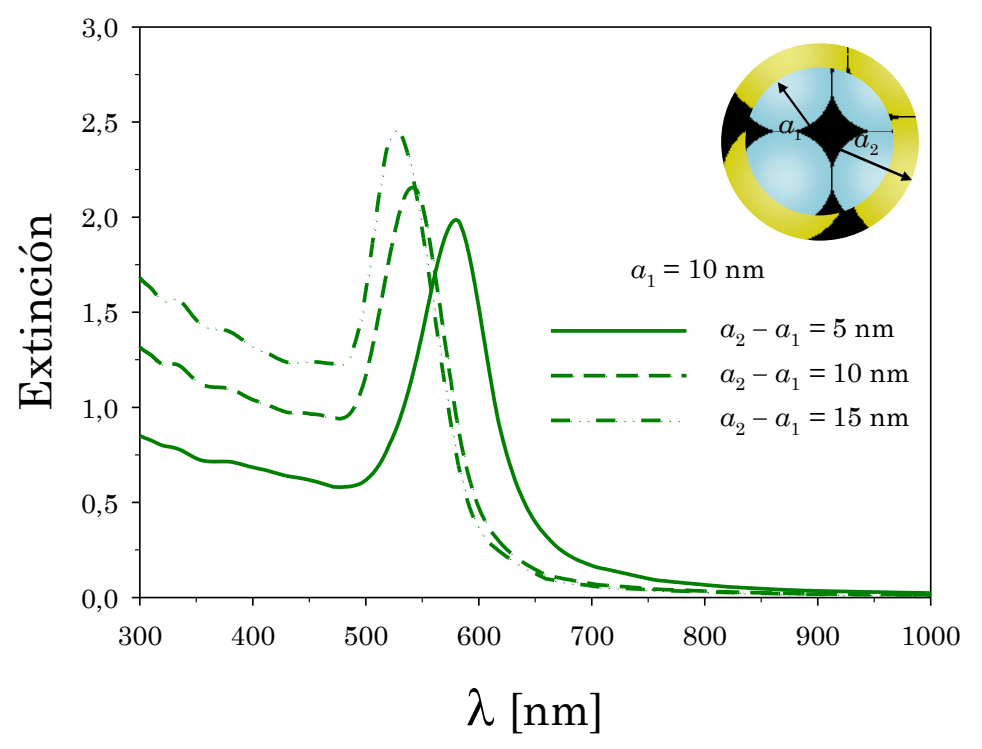

Figura 2.6: Espectros de extinción calculados para una esfera de $\mathrm{SiO}_{2}$ de $10 \mathrm{~nm}$ de radio de núcleo con tres espesores de recubrimiento de oro de $5 \mathrm{~nm}, 10 \mathrm{~nm}$ y $15 \mathrm{~nm}$ en agua. Los datos de la función dieléctrica del oro y del $\mathrm{SiO}_{2}$ fueron tomados de Johnson y Christy [Johnson and Christy 1972] y Palik [Palik 1985], respectivamente. 
En el caso de nanopartículas metálicas, para ambas estructuras, la función dieléctrica compleja metálica presenta una dependencia con el tamaño de la partícula, por lo que se debe realizar una adecuada modificación de la función dieléctrica, para luego poder calcular el coeficiente de extinción. Este procedimiento se desarrollará en el Capítulo 3. 


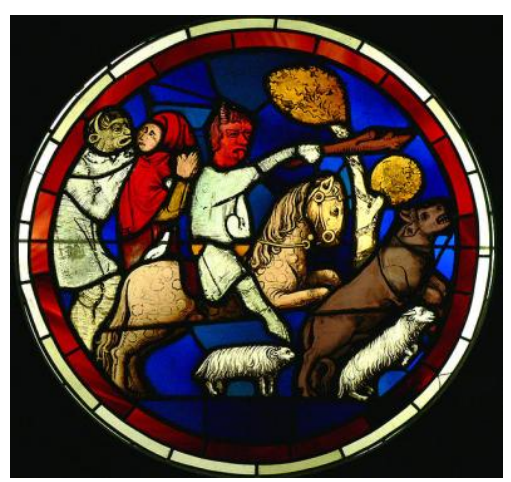

Santa Capilla

\section{Capítulo 3}

París, Francia

\section{Modelado de la función dieléctrica de metales}

Los fenómenos ópticos de refracción, reflexión, absorción, luminiscencia y scattering que se observan cuando una onda electromagnética interactúa con materiales sólidos pueden ser cuantificados con un cierto número de parámetros tales como la reflectividad, transmitividad, scattering, entre otros y determinan las propiedades del medio a nivel macroscópico. En el caso de los metales, el aspecto brillante de su superficie está íntimamente ligado al tipo de interacción que existe entre una onda electromagnética incidente y los electrones libres del material. Estos fenómenos se pueden interpretar con un modelo simple para caracterizar la estructura microscópica de la materia.

En este capítulo se realiza una breve introducción a las ecuaciones de Maxwell y las relaciones constitutivas de la materia, para luego plantear el modelado microscópico de la función dieléctrica de metales. 


\subsection{Ecuaciones de Maxwell y relaciones constitutivas}

En la descripción electrodinámica macroscópica, el carácter discreto de las cargas y de las corrientes asociadas a ellas es reemplazado por los conceptos de densidad de carga $\rho$ y densidad de corriente $\mathbf{j}$ que se toman como funciones continuas de las coordenadas espaciales. Asimismo, los campos eléctricos y magnéticos son considerados promedios espaciales locales de los campos microscópicos asociados a las cargas discretas y también se toman como funciones continuas. Las expresiones diferenciales de las ecuaciones de Maxwell macroscópicas en el sistema de unidades SI tienen la forma:

$$
\begin{gathered}
\nabla \times \mathbf{E}(\mathbf{r}, t)=-\frac{\partial \mathbf{B}(\mathbf{r}, t)}{\partial t} \\
\nabla \times \mathbf{H}(\mathbf{r}, t)=\frac{\partial \mathbf{D}(\mathbf{r}, t)}{\partial t}+\mathbf{j}(\mathbf{r}, t) \\
\nabla \cdot \mathbf{D}(\mathbf{r}, t)=\rho(\mathbf{r}, t) \\
\nabla \cdot \mathbf{B}(\mathbf{r}, t)=0
\end{gathered}
$$

donde $\mathbf{E}$ es el campo eléctrico, $\mathbf{D}$ es el desplazamiento eléctrico, $\mathbf{H}$ es el campo magnético, $\mathbf{B}$ es la inducción eléctrica, $\mathbf{j}$ es la densidad de corriente y $\rho$ es la densidad de carga.

Las propiedades electromagnéticas del medio se describen en función del vector polarización macroscópica $\mathbf{P}$ y del vector magnetización $\mathbf{M}$ de acuerdo a las siguientes relaciones:

$$
\mathbf{D}(\mathbf{r}, t)=\varepsilon_{0} \mathbf{E}(\mathbf{r}, t)+\mathbf{P}(\mathbf{r}, t)
$$




$$
\mathbf{H}(\mathbf{r}, t)=\mu_{0}^{-1} \mathbf{B}(\mathbf{r}, t)-\mathbf{M}(\mathbf{r}, t)
$$

donde $\varepsilon_{0} \mathrm{y} \mu_{0}$ son la permitividad eléctrica y la permeabilidad magnética en el vacío, respectivamente.

Si bien las ecuaciones de Maxwell relacionan los campos producidos por las cargas y las corrientes en la materia, no describen de qué manera son generadas estas últimas. Para encontrar una solución autoconsistente para el campo electromagnético, las ecuaciones de Maxwell deben ser complementadas por relaciones que describen el comportamiento de la materia bajo la influencia de los campos. Estas ecuaciones se conocen como relaciones constitutivas. Para el caso de un medio isótropo, homogéneo y no dispersivo, toman la forma:

$$
\begin{array}{cr}
\mathbf{D}=\varepsilon_{0} \varepsilon \mathbf{E} & \mathbf{P}=\varepsilon_{0} \chi_{\mathrm{e}} \mathbf{E} \\
\mathbf{B}=\mu_{0} \mu \mathbf{H} \quad \mathbf{M}=\chi_{\mathrm{m}} \mathbf{H} \\
\mathbf{j}_{\mathrm{c}}=\sigma \mathbf{E}
\end{array}
$$

donde $\chi_{\mathrm{e}} \mathrm{y} \quad \chi_{\mathrm{m}}$ representan la susceptibilidad eléctrica y magnética, respectivamente, $\sigma$ es la conductividad eléctrica y $\quad \varepsilon$ y $\mu$ son la susceptibilidad eléctrica y magnética del medio, respectivamente.

Un campo electromagnético arbitrario que se propaga en un medio lineal puede ser escrito como una superposición de campos monocromáticos de la forma:

$$
\mathbf{E}(\mathbf{r}, t)=\mathbf{E}(\mathbf{k}, \omega) \cos (\mathbf{k} \cdot \mathbf{r}-\omega t)
$$


donde $\mathbf{k}$ y $\omega$ son el vector de onda y la frecuencia angular del campo, respectivamente. Así, la amplitud del vector desplazamiento puede ser escrita como:

$$
\mathbf{D}(\mathbf{k}, \omega)=\varepsilon_{0} \varepsilon(\mathbf{k}, \omega) \mathbf{E}(\mathbf{k}, \omega)
$$

donde ahora la función dieléctrica es función del vector de onda y de la frecuencia del campo.

\subsection{Función dieléctrica macroscópica para metales}

Las propiedades ópticas de los metales pueden describirse por una función dieléctrica compleja que depende de la frecuencia de la luz $(\omega)$, y está determinada principalmente por dos razones:

(i) los electrones de conducción se pueden mover dentro del volumen del material;

(ii) las transiciones interbanda tienen lugar cuando la energía de los fotones excede la energía de la banda prohibida del metal.

Un campo eléctrico que produce un desplazamiento d de un electrón, genera un momento dipolar $\mathbf{p}=e \mathbf{d}$. El efecto acumulativo de los momentos dipolares individuales de todos los electrones libres da como resultado una polarización macroscópica por unidad de volumen $\mathbf{P}=n_{e} \mathbf{p}$, siendo $n_{e}$ el número de electrones por unidad de volumen, que puede expresarse como

$$
\mathbf{P}(\omega)=\varepsilon_{0} \chi_{\mathrm{e}}(\omega) \mathbf{E}(\omega)
$$


donde $\chi_{\mathrm{e}}$ es la susceptibilidad eléctrica del material.

Utilizando las ecuaciones de Maxwell y las relaciones constitutivas, cuando se considera un medio lineal, homogéneo e isótropo, se tiene

$$
\mathbf{D}(\omega)=\varepsilon_{0} \varepsilon(\omega) \mathbf{E}(\omega)=\varepsilon_{0} \mathbf{E}(\omega)+\mathbf{P}(\omega)
$$

De esta ecuación se puede obtener la siguiente expresión

$$
\varepsilon(\omega)=1+\chi_{\mathrm{e}}(\omega)
$$

donde $\varepsilon(\omega)$ es la función dieléctrica del metal dependiente de la frecuencia, que se puede escribir como: $\varepsilon(\omega)=\varepsilon^{\prime}(\omega)+i \varepsilon^{\prime \prime}(\omega)$. El desplazamiento $\mathbf{d}$ y por lo tanto la polarización macroscópica $\mathbf{P}$ y $\chi_{\mathrm{e}}$ pueden ser obtenidos resolviendo la ecuación de movimiento de los electrones bajo la influencia de un campo externo.

La Figura 3.1 muestra un ejemplo del comportamiento de las partes real (a) e imaginaria (b) de la función dieléctrica macroscópica (bulk) experimental del oro como función de la longitud de onda.

En general, esta función dieléctrica compleja bulk de metales se puede descomponer en dos términos, un término complejo de los electrones libres y otro término complejo de las transiciones interbanda (o electrones ligados). Dado que la función dieléctrica es aditiva [Bohren and Huffman 1998], puede escribirse como:

$$
\varepsilon(\omega)=\varepsilon_{\text {electrones-libres }}(\omega)+\varepsilon_{\text {electrones -ligados }}(\omega)=\varepsilon^{\prime}(\omega)+i \varepsilon^{\prime \prime}(\omega)
$$

Por lo tanto, las partes real e imaginaria de la función dieléctrica contienen contribuciones de los electrones libres y ligados, respectivamente. 

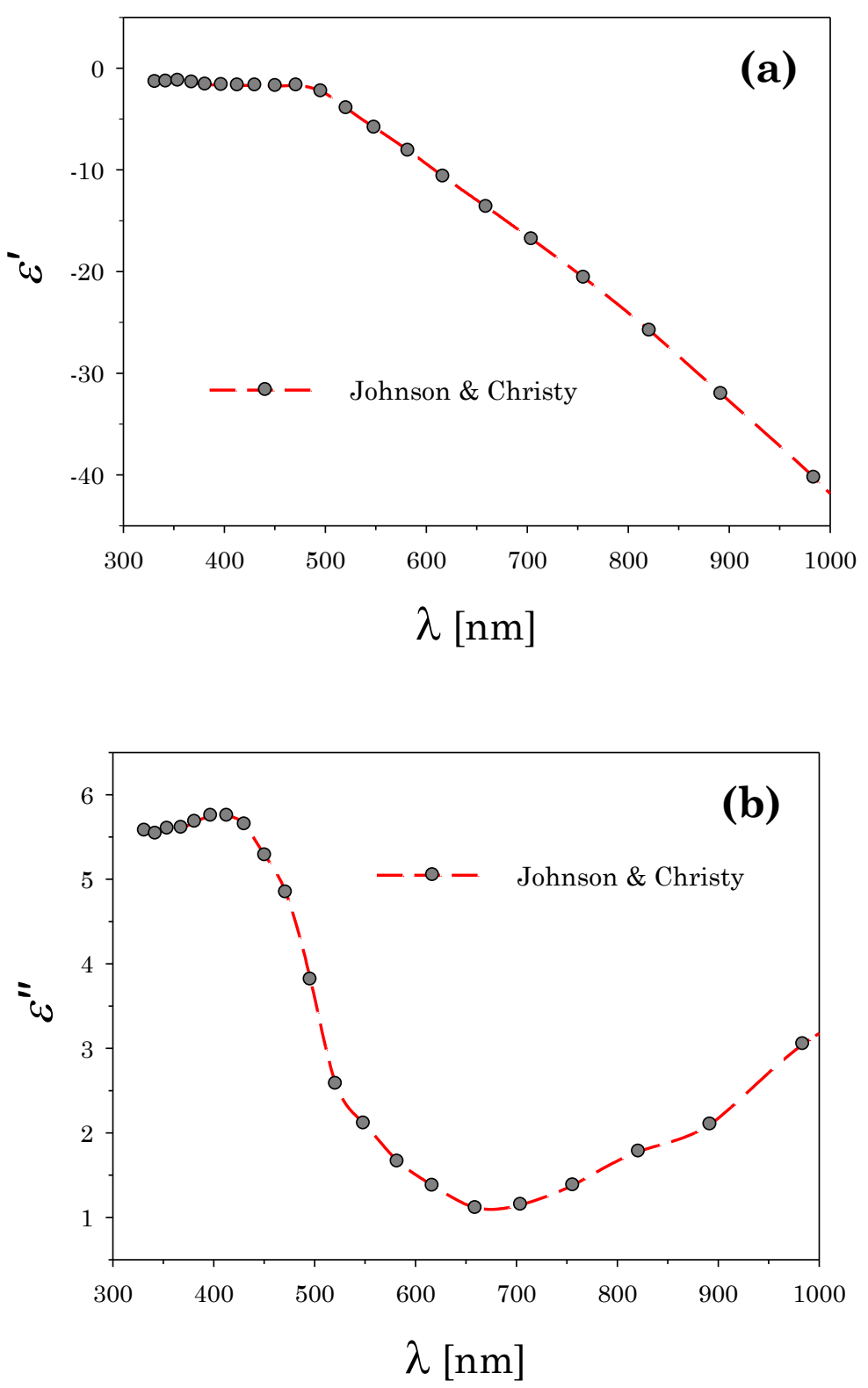

Figura 3.1: (a) Parte real y (b) parte imaginaria de la función dieléctrica bulk del oro. Los valores experimentales fueron tomados de Johnson y Christy [Johnson and Christy 1972].

La función dieléctrica para los sistemas nanométricos no sólo es una función de la longitud de onda (o frecuencia) de la radiación incidente, sino que también es sensible al tamaño y forma de la partícula, [Kottmann and Martin 2001, Kelly et al. 2003, Coronado et al. 2003, Ranjan et al. 2010]. 
La expresión de la función dieléctrica para una partícula en la escala nanométrica no es trivial. Existen diferentes aproximaciones que pueden ser utilizadas, tales como el modelo de jellium o el modelo de química-cuántica [Bonacic-Koutecky et al. 1991, Brack 1993], basados en las consideraciones relacionadas con el comportamiento colectivo de los electrones o en los cálculos de primeros principios, respectivamente. Sin embargo, si se realizan correcciones adecuadas y físicamente significativas, el concepto clásico de la función dieléctrica puede ampliarse a tamaños nanométricos. Este enfoque produce resultados que se ajustan satisfactoriamente a los datos experimentales y a la vez dan una visión física de los procesos electrónicos que son útiles para la determinación de parámetros vinculados a la contribución de los electrones ligados.

\subsubsection{Modelo de Lorentz}

En un primer modelo clásico simple de la materia, los electrones se encuentran unidos a los núcleos por fuerzas restauradoras lineales (modelo de Lorentz) [Lorentz 1905], por lo cual se los considera como osciladores armónicos clásicos con una frecuencia propia $\omega_{0}$. La ecuación de movimiento de estos osciladores armónicos amortiguados forzados toma la forma:

$$
m_{l} \frac{\partial^{2} \mathbf{d}}{\partial t^{2}}+m_{l} \gamma \frac{\partial \mathbf{d}}{\partial t}+\kappa \mathbf{d}=e \mathbf{E}_{0} e^{-i \omega t}
$$

donde $m_{l}$ y $\gamma$ son la masa y la constante de amortiguamiento de los osciladores, respectivamente, y $\kappa$ es la constante de la fuerza restauradora que mantiene al electrón en su lugar. 
Para este modelo, la función dieléctrica relativa $\varepsilon$, definida como $\varepsilon=\epsilon / \varepsilon_{0}$, correspondiente a un conjunto de $\boldsymbol{N}$ osciladores armónicos amortiguados por unidad de volumen, cada uno con frecuencia de resonancia $\omega_{0}$, se escribe como:

$$
\varepsilon=1+\frac{\omega_{p}^{2}}{\omega_{0}^{2}-\omega^{2}-i \gamma \omega}
$$

donde $\omega_{p}$ es la frecuencia de plasma definida en la ecuación (1.1).

Las propiedades ópticas de los materiales se describen alternativamente por dos conjuntos de cantidades: las partes real e imaginaria del índice de refracción complejo $N=n+i k$, o las partes real e imaginaria de la función dieléctrica $\varepsilon=\varepsilon^{\prime}+i \varepsilon^{\prime \prime}$. Para medios no magnéticos, se mantiene la relación $N=\sqrt{\varepsilon}$. Así, las partes real e imaginaria de ambos parámetros están relacionados por:

$$
\begin{gathered}
\varepsilon^{\prime}=n^{2}-k^{2} \\
\varepsilon^{\prime \prime}=2 n k
\end{gathered}
$$

\subsubsection{Contribución de los electrones libres}

Los estados de energía de los electrones en materiales sólidos se pueden representar por un continuo de los niveles de energía agrupados en bandas diferentes. Para los metales, la banda superior ocupada está parcial o completamente llena de electrones y se superpone con una banda superior vacía, como se muestra en la Figura 3.2 en una forma simplificada. 


\section{METALES}

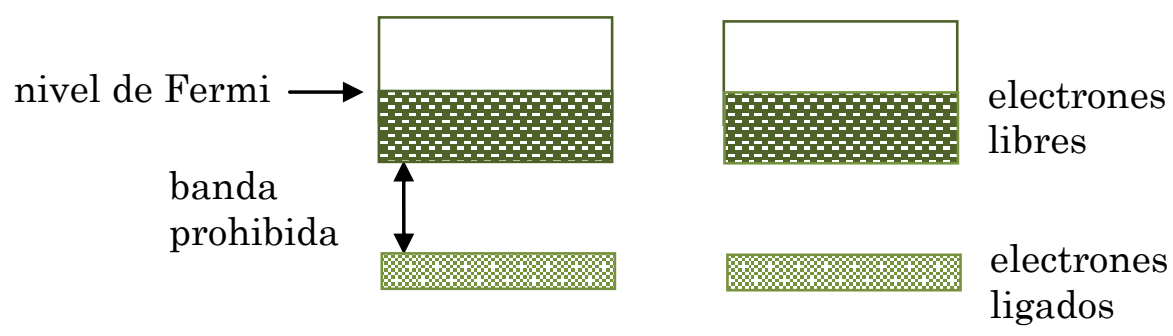

Figure 3.2: Representación simplificada de las bandas de energía de los electrones de metales sólidos.

Los electrones en los metales de tamaño macroscópico son considerados esencialmente como "libres", ya que pueden ser promovidos a los niveles de energía vacíos más altos (desde la parte superior de la distribución de energía, cerca del nivel de Fermi) con energías fotónicas muy pequeñas. En este sentido, se puede considerar que los electrones no están ligados a los iones de la red, por lo que su frecuencia de resonancia puede ser igual a cero en el modelo de Lorentz [Drude 1900a, Drude 1900b]. Para este caso, la ecuación clásica de movimiento de los electrones libres con amortiguamiento bajo la acción de un campo eléctrico externo de amplitud $\mathbf{E}_{0}$ y frecuencia $\omega$ es:

$$
m_{e} \frac{\partial^{2} \mathbf{d}}{\partial t^{2}}+m_{e} \gamma_{l i b r e s} \frac{\partial \mathbf{d}}{\partial t}=e \mathbf{E}_{0} e^{-i \omega t}
$$

donde $e$ y $m_{e}$ son la carga y la masa efectiva de los electrones libres, respectivamente. La constante de amortiguamiento $\gamma_{\text {libres }}$ es proporcional a $v_{F} / l$ donde $v_{F}$ es la velocidad de Fermi y $l$ es el camino libre medio de los 
electrones entre dos eventos de scattering. Por lo tanto, la expresión para la función dieléctrica de un "metal de electrones libres" se obtiene de acuerdo al modelo de Drude-Lorentz, sustituyendo $\omega_{0}=0$ en la ecuación (3.17):

$$
\varepsilon_{\text {libres }}(\omega)=1-\frac{\omega_{p}^{2}}{\omega^{2}+i \omega \gamma_{\text {libres }}}
$$

con sus partes real e imaginaria dadas por:

$$
\begin{gathered}
\varepsilon_{\text {libres }}^{\prime}(\omega)=1-\frac{\omega_{p}^{2}}{\omega^{2}+\gamma_{\text {libres }}^{2}} \\
\varepsilon_{\text {libres }}^{\prime \prime}(\omega)=\frac{\omega_{p}^{2} \gamma_{\text {libres }}}{\omega\left(\omega^{2}+\gamma_{\text {libres }}^{2}\right)}
\end{gathered}
$$

La frecuencia de plasma $\omega_{p}$ se encuentra en la región ultravioleta del espectro y $\hbar \omega_{p}$ en el rango de 4 a $20 \mathrm{eV}$. En el metal de dimensiones macroscópicas, la constante de amortiguamiento $\gamma_{\text {libres }}$, tiene una simple interpretación física en términos del tiempo total promedio $\tau$ entre las colisiones de los electrones libres con otros electrones (scattering electrónelectrón), las vibraciones de la red (scattering electrón-fonón) y las imperfecciones. Este tiempo $\tau$ se expresa como:

$$
\gamma_{\text {libres }}=\frac{1}{\tau}=\frac{1}{\tau_{e l-e l}}+\frac{1}{\tau_{e l-f o}}+\frac{1}{\tau_{i m}}
$$

En las Figuras 3.3 (a) y (b) se muestran las partes real e imaginaria de la función dieléctrica bulk de los electrones libres para oro, utilizando las expresiones (3.22) y (3.23). 

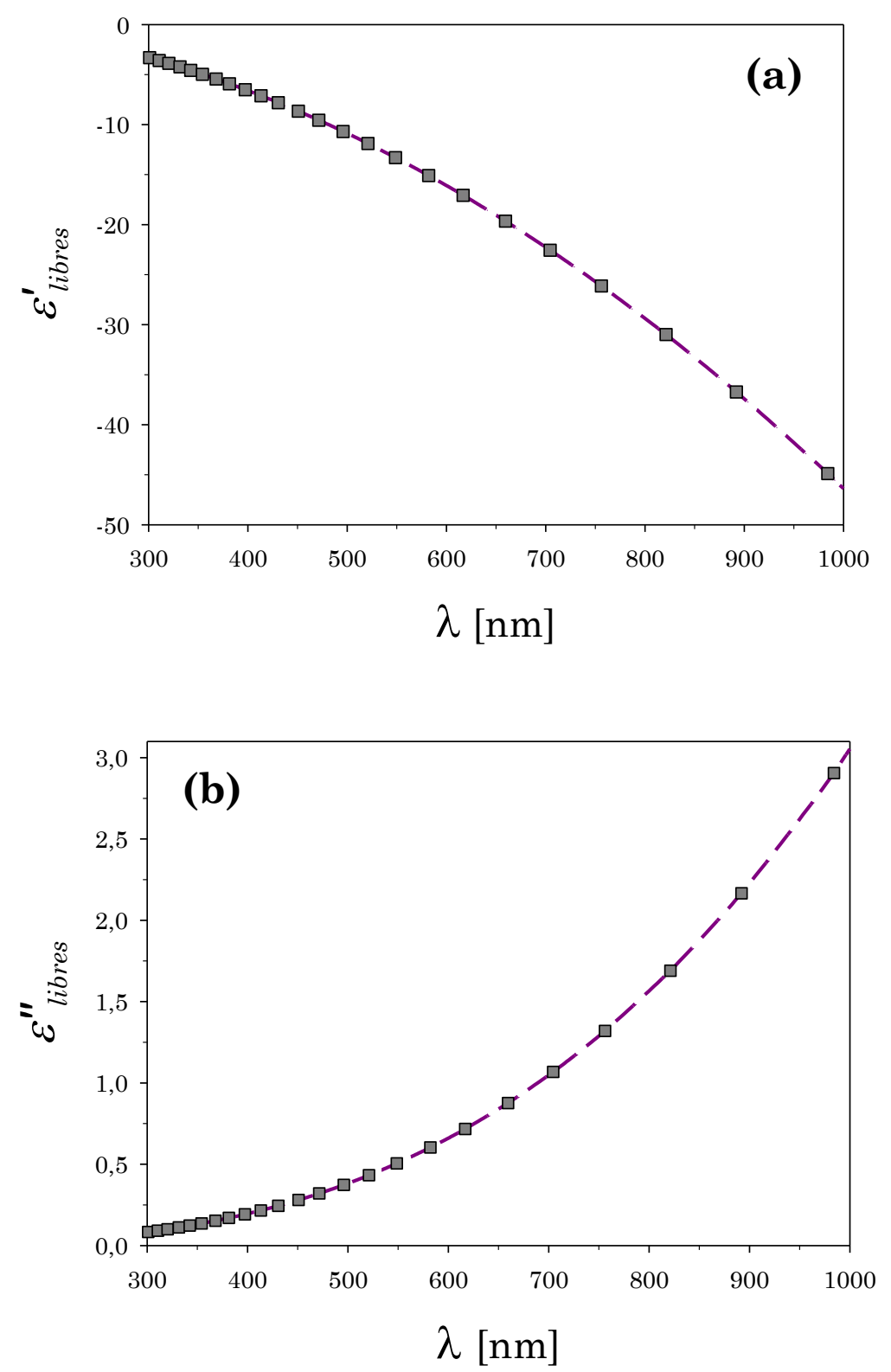

Figura 3.3: (a) Parte real y (b) parte imaginaria de la función dieléctrica de los electrones libres del oro. Los datos experimentales fueron tomados de Johnson y Christy.

\subsubsection{Contribución de los electrones ligados}

Como se mencionó en la Sección 3.2., la expresión completa de la función dieléctrica compleja está formada por una componente 
correspondiente a los electrones libres y otra a los electrones ligados (ecuación (3.15)). En principio, la contribución de los electrones ligados se puede obtener restando la función dieléctrica compleja bulk del metal (3.15) y la expresión compleja (3.21) de los electrones libres, dando como resultado:

$$
\varepsilon_{\text {ligados }}(\omega)=\varepsilon(\omega)-1+\frac{\omega_{p}^{2}}{\omega^{2}+i \omega \gamma_{\text {libres }}}
$$

donde los datos experimentales de $\varepsilon(\omega)$ son tomados de Johnson y Christy [Johnson and Christy 1972] o Palik [Palik 1985]. Esta función dieléctrica de los electrones ligados se descompone en sus partes real e imaginaria dando:

$$
\begin{gathered}
\varepsilon_{\text {ligados }}^{\prime}(\omega)=\varepsilon^{\prime}(\omega)-1+\frac{\omega_{p}^{2}}{\omega^{2}+\gamma_{\text {libres }}^{2}} \\
\varepsilon_{\text {ligados }}^{\prime \prime}(\omega)=\varepsilon^{\prime \prime}(\omega)-\frac{\omega_{p}^{2} \gamma_{\text {libres }}}{\omega\left(\omega^{2}+\gamma_{\text {libres }}^{2}\right)}
\end{gathered}
$$

Para el caso de un metal de tamaño macroscópico, tanto la función dieléctrica de los electrones libres como la de los ligados no son dependientes del tamaño, por lo tanto para calcular la función dieléctrica compleja de una partícula metálica grande se utilizan simplemente las ecuaciones (3.18) y (3.19), con los datos experimentales del índice de refracción complejo.

En las Figuras 3.4 (a) y (b) se observan las partes real e imaginaria de la función dieléctrica bulk de los electrones ligados para oro, calculadas con las ecuaciones (3.26) y (3.27). 

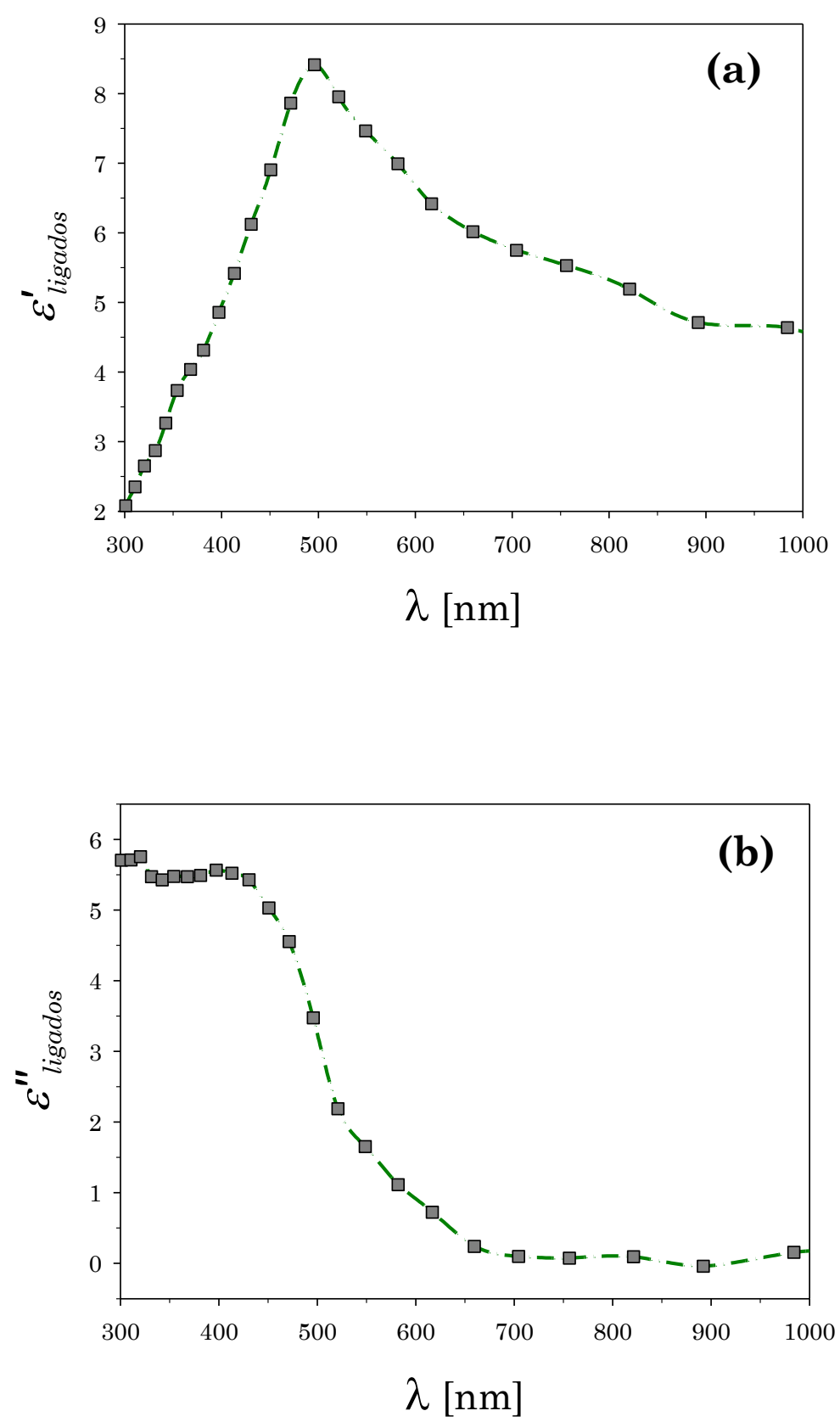

Figura 3.4: (a) Parte real y (b) parte imaginaria de la función dieléctrica de los electrones ligados del oro. Los valores experimentales fueron tomados de Johnson y Christy. 


\subsection{Función dieléctrica de partículas nanométricas $(a<10 \mathrm{~nm})$}

Para partículas nanométricas más pequeñas que el camino libre medio del electrón (unas pocas decenas de nanómetros para metales de tamaño macroscópico), la colisión de los electrones con los bordes de la partícula (superficie) tiene mayor importancia. Este proceso reduce el camino libre medio de los electrones, y a su vez conduce a un incremento de la parte imaginaria de la función dieléctrica. Esto se ha comprobado experimentalmente para nanopartículas esféricas [Doyle 1958, Kraus and Schatz 1983a, Kraus and Schatz 1983b, Kreibig and Vollmer 1995, Scaffardi et al. 2005]. En estos trabajos se demuestra que para tamaños de partículas inferiores a $10 \mathrm{~nm}$ el ancho de la resonancia se hace más amplio.

En este caso, la componente de los electrones libres de la función dieléctrica compleja bulk del metal se vuelve dependiente del tamaño, pero la componente de los electrones ligados aún se la considera independiente del tamaño de la partícula.

\subsubsection{Corrección de los electrones libres por tamaño}

Para partículas pequeñas, la frecuencia de plasma se asume como independiente del tamaño, mientras que la constante de amortiguamiento, relacionada con el camino libre medio de los electrones, está fuertemente afectada por el tamaño. Este hecho se tiene en cuenta al incluir un término aditivo en la ecuación (3.24), que debe ser inversamente proporcional al tiempo medio entre las colisiones de los electrones con el borde de la partícula $1 / \tau_{a}=C\left(v_{F} / a\right)$, donde $v_{F}$ es la velocidad de los electrones en el nivel de Fermi y a es el radio de la nanopartícula. La constante de 
proporcionalidad $C$ tiene en cuenta los detalles de los procesos de scattering del electrón con el borde de la partícula y puede ser calculada a partir de la teoría electrodinámica o del modelo cuántico de la caja con paredes de potencial infinito [Genzel et al. 1975, Ruppin and Yatom 1976, Wood and Ashcroft 1982, Apell and Penn 1983]. Para esferas, su valor varía entre 0,75 y 1,15 [Kreibig and Vollmer 1995].

Con estas consideraciones, se puede introducir una constante de amortiguamiento dependiente del tamaño que se expresa como:

$$
\gamma_{\text {libres }}^{t}=\gamma_{\text {libres }}+C \frac{v_{F}}{a}
$$

El segundo término de esta ecuación se vuelve despreciable para radios grandes $(a>10 \mathrm{~nm})$, y por lo tanto la constante de amortiguamiento de tamaño $\left(\gamma_{\text {libres }}^{t}\right)$ tiende a un valor macroscópico. Entonces, para $a<10 \mathrm{~nm}$ las ecuaciones (3.22) y (3.23) toman la forma:

$$
\begin{aligned}
& \varepsilon_{\text {libres }}^{\prime}(\omega, a)=1-\frac{\omega_{p}^{2}}{\omega^{2}+\left(\gamma_{\text {libres }}^{t}\right)^{2}} \\
& \varepsilon_{\text {libres }}^{\prime \prime}(\omega, a)=\frac{\omega_{p}^{2} \gamma_{\text {libres }}^{t}}{\omega\left(\omega^{2}+\left(\gamma_{\text {libres }}^{t}\right)^{2}\right)}
\end{aligned}
$$

Usando las ecuaciones (3.29) y (3.30), se puede obtener un conjunto de curvas de las partes real e imaginaria de la función dieléctrica para diferentes radios. Las Figuras 3.5, 3.6 y 3.7 muestran un ejemplo para el caso del oro $(\mathrm{Au})$, de la plata $(\mathrm{Ag})$ y del cobre $(\mathrm{Cu})$, respectivamente, cuando se representa en función de la longitud de onda. 

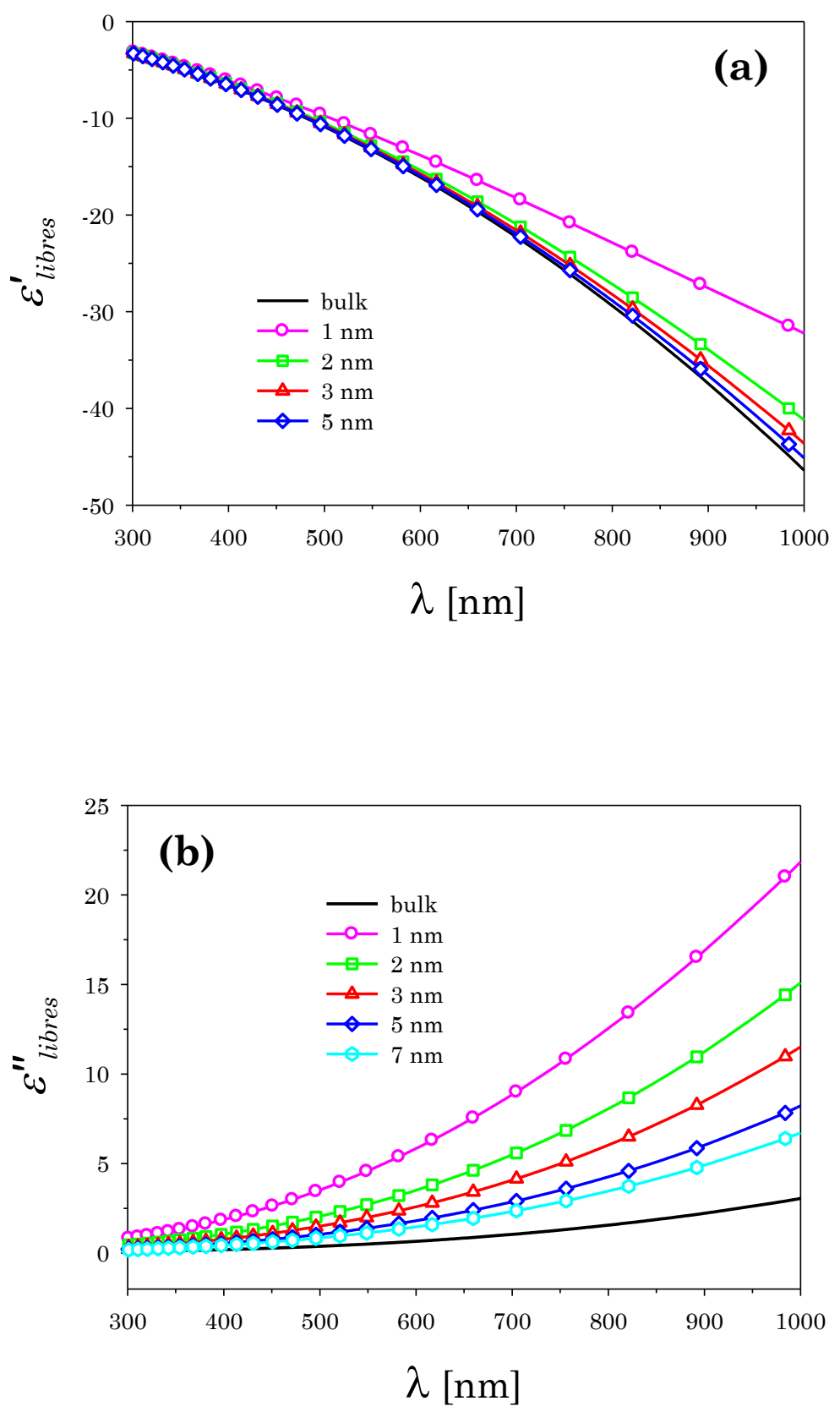

Figura 3.5: (a) Parte real y (b) parte imaginaria de la función dieléctrica de los electrones libres del oro calculadas con las ecuaciones (3.29) y (3.30). Los valores usados son: $v_{F}=14,1 \times 10^{14} \mathrm{~nm} / \mathrm{s}$ y $C=0,8$ [Granqvist and Hunderi 1977]. 

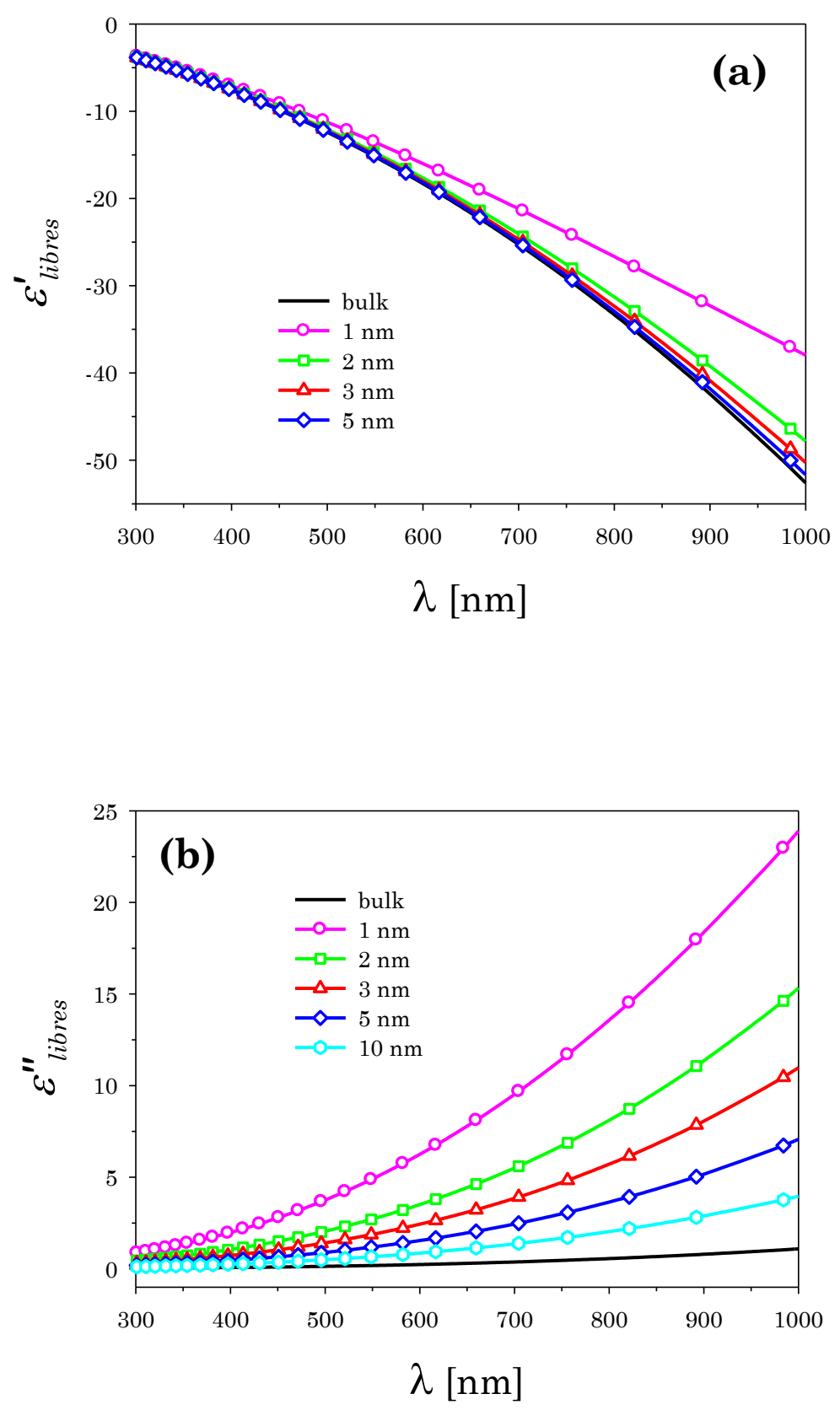

Figura 3.6: (a) Parte real y (b) parte imaginaria de la función dieléctrica de los electrones libres de la plata calculadas con las expresiones (3.29) y (3.30). Los valores usados son: $v_{F}=14,1 \times 10^{14} \mathrm{~nm} / \mathrm{s}$ y $C=0,8$ [Kittel 2007]. 

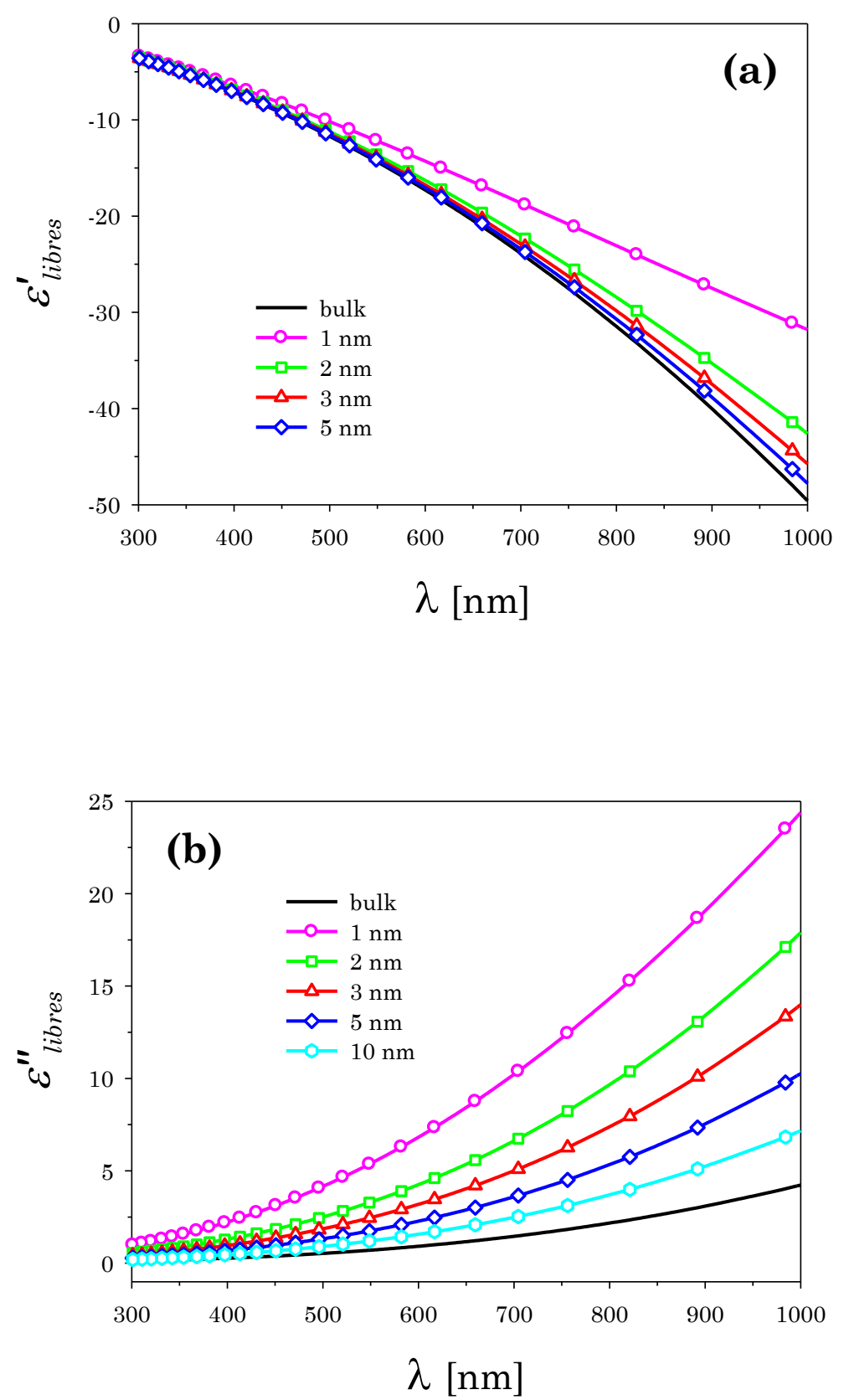

Figura 3.7: (a) Parte real y (b) parte imaginaria de la función dieléctrica de los electrones libres del cobre calculadas con las expresiones (3.29) y (3.30). Los valores usados son: $v_{F}=15,7 \times 10^{14} \mathrm{~nm} / \mathrm{s}$ y $C=0,8$ [Kaye and Laby 1995]. 
Se puede observar que cada conjunto de curvas, dependiente de la longitud de onda, tiende a una curva común (bulk) a medida que el radio aumenta, pero presenta claras diferencias para tamaños que van de 1 a $10 \mathrm{~nm}$, aproximadamente, dependiendo del metal. Entonces se demuestra que es posible reproducir valores macroscópicos de la función dieléctrica por un simple cálculo de las expresiones (3.29) y (3.30) para un radio $100 \mathrm{~nm}$, ya que para estos tamaños el segundo término en la ecuación (3.28) es despreciable. En esta aproximación del electrón libre, todos los metales nobles ( $\mathrm{Au}, \mathrm{Ag}$ y $\mathrm{Cu}$ ) se comportan de la misma forma y sus funciones dieléctricas son muy similares, excepto por las pequeñas diferencias que surgen de los diferentes valores de la frecuencia de plasma.

\subsubsection{Inclusión de la corrección de electrones libres en la función dieléctrica}

En este caso, la contribución de los electrones ligados sigue siendo una función independiente del tamaño (ecuación (3.25)), mientras que la contribución de los electrones libres para nanopartículas con tamaño menor a $10 \mathrm{~nm}$ es dependiente del tamaño, como se ha calculado en la Sección 3.3.1. Como consecuencia la función dieléctrica compleja total se vuelve dependiente del tamaño, y se puede escribir como:

$$
\varepsilon(\omega, a)=\varepsilon_{\text {libres }}(\omega, a)+\varepsilon_{\text {ligados }}(\omega)
$$

donde $\varepsilon_{\text {libres }}(\omega, a)$ se obtiene con las expresiones (3.29) y (3.30).

Las Figuras 3.8, 3.9 y 3.10 muestran, las partes real (a) e imaginaria (b) de la función dieléctrica completa de oro, plata y cobre, respectivamente para cada metal de tamaño macroscópico [Johnson and Christy 1972], y 
para diferentes radios de nanopartículas calculadas a partir de la ecuación (3.31).
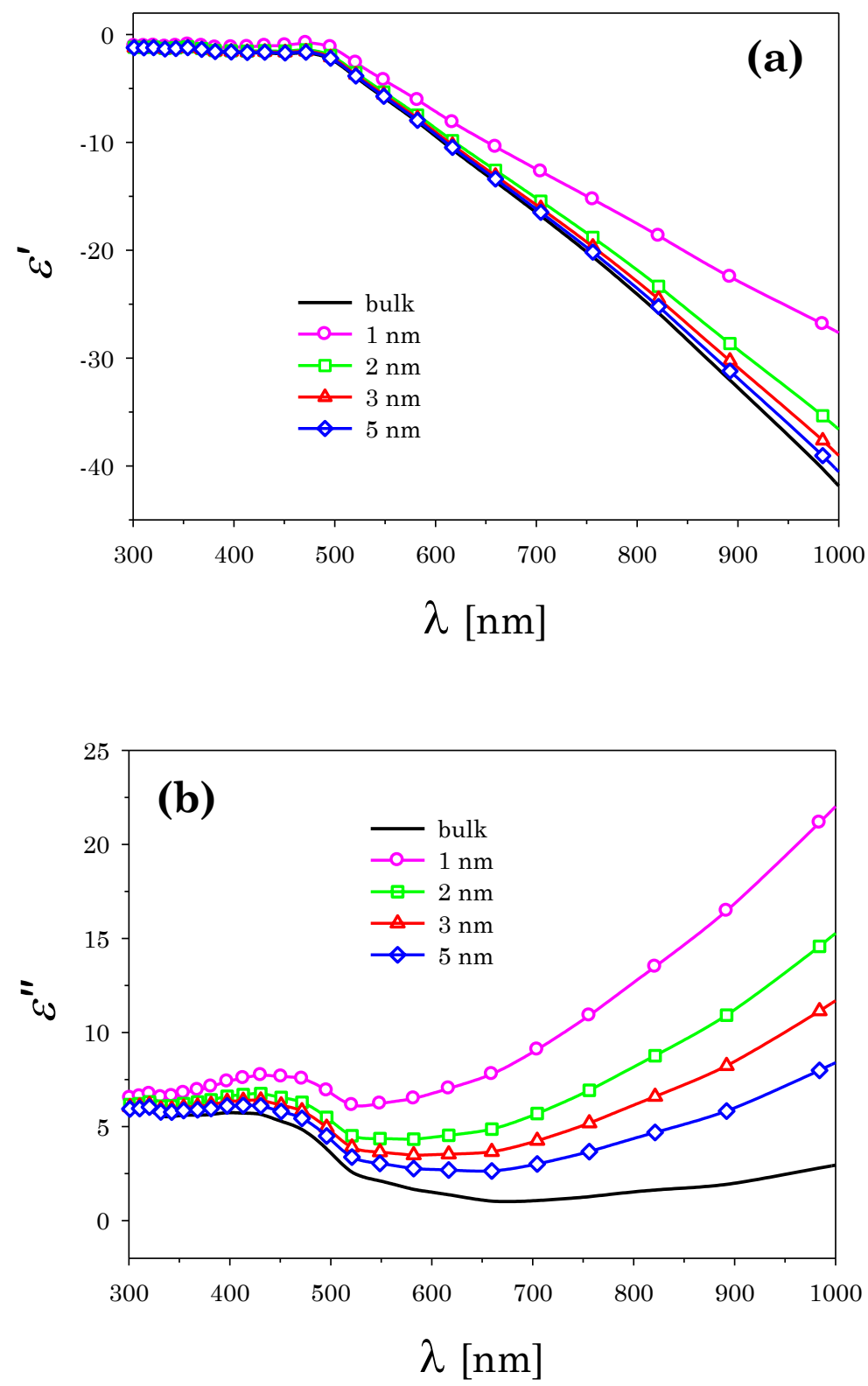

Figura 3.8: (a) Parte real y (b) parte imaginaria de la función dieléctrica completa del oro de acuerdo a la expresión (3.31). La curva negra representa los datos experimentales tomados de Johnson y Christy. Las curvas en color representan la corrección para partículas de 1 a $5 \mathrm{~nm}$ de radio [Pinchuk et al. 2004]. 

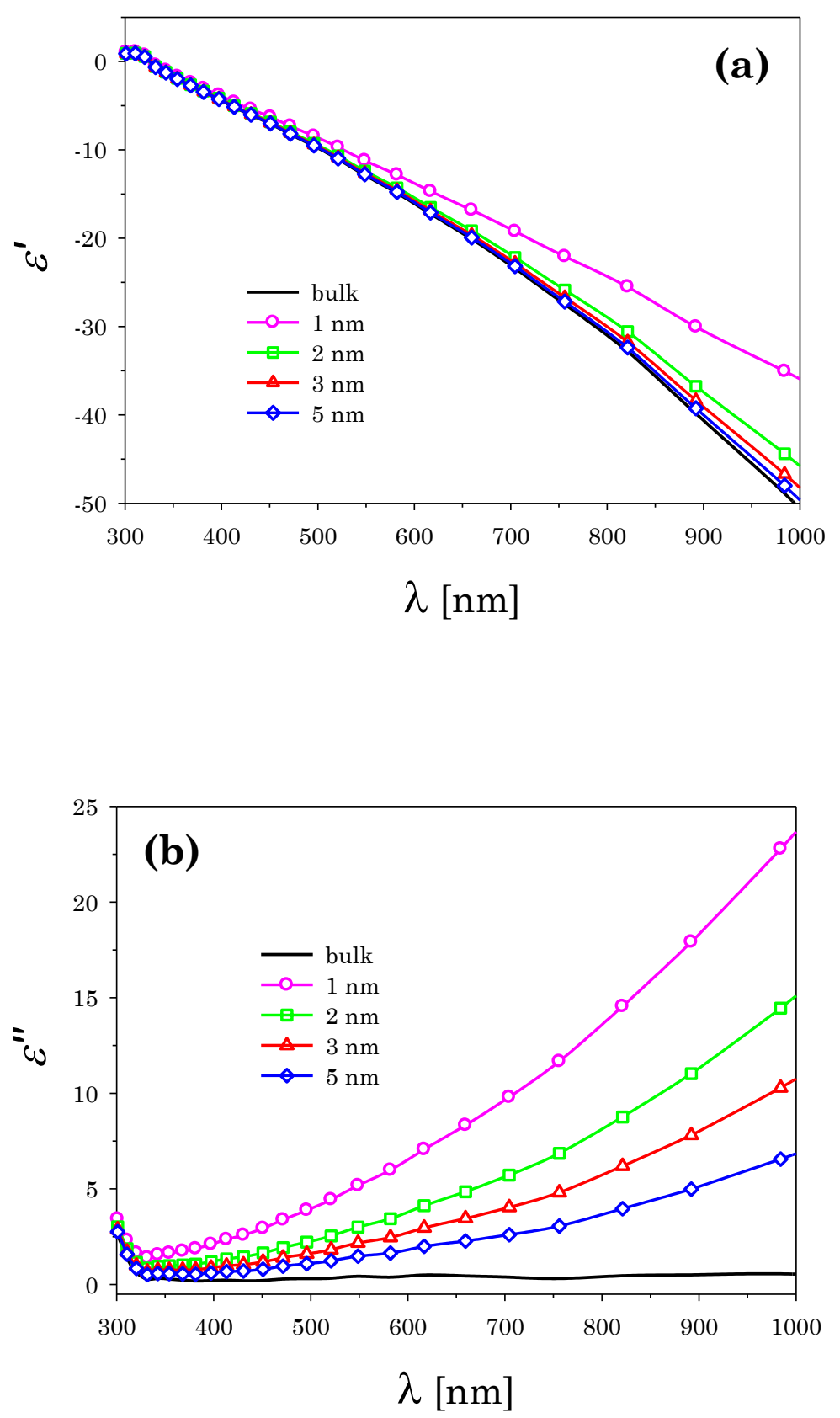

Figura 3.9: (a) Parte real y (b) parte imaginaria de la función dieléctrica completa de plata calculada con la expresión (3.31). La curva negra representa los valores experimentales tomados de Johnson y Christy. En color se representa la corrección para partículas de 1 a $5 \mathrm{~nm}$ de radio [Scaffardi et al. 2007]. 

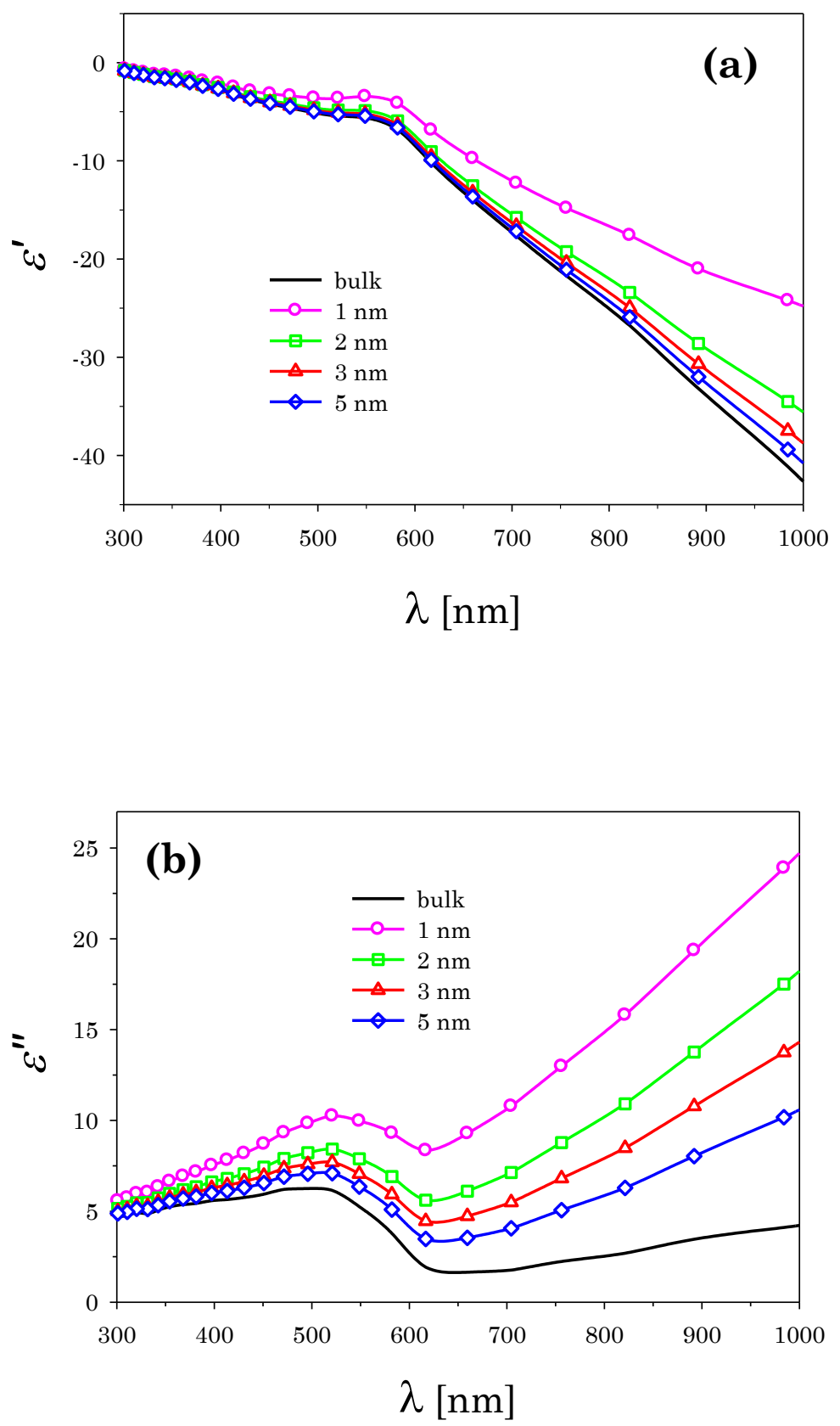

Figura 3.10: (a) Parte real y (b) parte imaginaria de la función dieléctrica completa del cobre de acuerdo a la ecuación (3.31). La curva negra representa los valores experimentales tomados de Johnson y Christy. Las curvas en color representan la corrección para partículas de 1 a $5 \mathrm{~nm}$ de radio. 
Se observa en la parte real un cambio abrupto de pendiente para una longitud de onda que depende del metal noble. La influencia de los electrones ligados está asociada a longitudes de onda menores que la correspondiente a este cambio de pendiente. Esto tiene más notoriedad en el comportamiento de la parte imaginaria, que muestra una resonancia ensanchada a longitudes de onda cortas.

\subsubsection{Contribución de los electrones ligados: transición única}

Cuando la energía del fotón incidente es lo suficientemente grande como para superar la banda prohibida y promover electrones de valencia (ligados) a la banda de conducción del material, estos electrones sumarán sus contribuciones a la función dieléctrica. Este tipo de transiciones se llaman transiciones electrónicas interbanda.

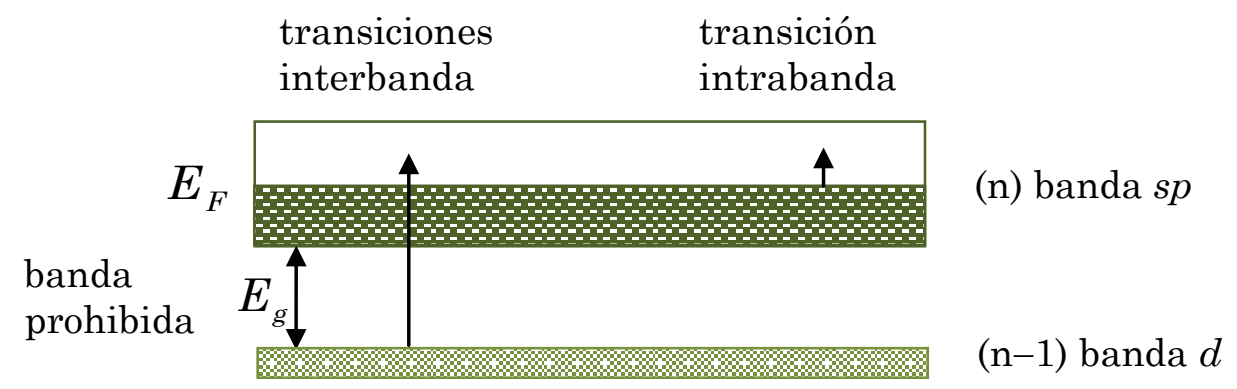

Figure 3.11: Esquema simplificado de las bandas de energía de los electrones de metales nobles. 
La Figura 3.11 muestra un diagrama simplificado de las bandas de energía de los electrones de un metal noble a cero grado Kelvin. Como la banda $s p$ se llena por encima de la energía de Fermi, las transiciones interbanda pueden ocurrir sobre un valor de energía umbral $\left(E_{g}+E_{F}\right)$. Las intrabanda que corresponden a los electrones libres pueden ser excitadas con fotones de baja energía.

El modelo de Drude-Lorentz descripto anteriormente tiene en cuenta sólo los electrones en las orbitas atómicas externas (por ejemplo los estados $5 s, 6 s$ y $4 s$ de plata, oro y cobre, respectivamente). Sin embargo, las transiciones interbanda, tales como la de la banda $5 d$ a la banda $6 s p$ en el oro, no puede ser ignorada cuando la frecuencia de la luz incidente cae en la región visible. Este efecto se puede tener en cuenta en $\varepsilon_{\text {ligados }}^{L}(\omega)$ por medio de una ecuación estándar como la de Lorentz [Coronado et al. 2003, Novotny and Hecht 2007, Vincenzo et al. 2011]:

$$
\varepsilon_{\text {ligados }}^{L}(\omega)=1+\frac{\omega_{p}^{\prime 2}}{\left(\omega_{0}^{2}-\omega^{2}\right)-i \omega \gamma_{\text {ligados }}}
$$

donde $\omega_{p}^{\prime}$ se introduce en analogía a la frecuencia de plasma en el modelo de Drude-Lorentz, $\gamma_{\text {ligados }}$ es la constante de amortiguamiento para el electrón ligado y $\omega_{0}$ es la frecuencia de resonancia del electrón ligado bajo la influencia del campo eléctrico aplicado. Las partes real e imaginaria están dadas por:

$$
\begin{gathered}
\varepsilon_{\text {ligados }}^{L L}(\omega)=1+\frac{\omega_{p}^{2}\left(\omega_{0}^{2}-\omega^{2}\right)}{\left(\omega_{0}^{2}-\omega^{2}\right)^{2}+\omega^{2} \gamma_{\text {ligados }}^{2}} \\
\varepsilon^{\prime \prime}{ }_{\text {ligados }}^{L}(\omega)=\frac{\omega_{p}^{\prime 2} \gamma_{\text {ligados }} \omega}{\left(\omega_{0}^{2}-\omega^{2}\right)^{2}+\omega^{2} \gamma_{\text {ligados }}^{2}}
\end{gathered}
$$


Dado que la función dieléctrica es aditiva, la expresión compleja se escribe como:

$$
\varepsilon(\omega, a)=\varepsilon_{\text {libres }}(\omega, a)+\varepsilon_{\text {ligados }}^{L}(\omega)
$$

La contribución total de los electrones libres y ligados a la función dieléctrica puede evaluarse a partir de la ecuación (3.35).

En la Figura 3.12, se grafican las partes real (a) e imaginaria (b) de la función dieléctrica del oro en el rango visible e infrarrojo cercano del espectro, usando la ecuación (3.35) con $a=100 \mathrm{~nm}$ para considerar un tamaño macroscópico. La función dieléctrica bulk, representada con círculos, es calculada a partir de los datos experimentales del índice de refracción complejo medidos por Johnson y Christy. La línea sólida corresponde a la contribución de los electrones libres, mientras que la línea discontinua representa la suma de la contribución de los electrones libres y una transición interbanda única (ligados) [Novotny and Hecht 2007]. En cuanto a la parte real, algunos autores agregan un término arbitrario constante, para ajustar los datos experimentales, llamado $\varepsilon_{\infty}$, cuyo valor oscila de 1 a 10 y representa las otras transiciones interbanda [Novotny and Hecht 2007, Maier 2007]. Para $\lambda>650 \mathrm{~nm}, \varepsilon(\omega, a)$ está gobernada por su parte real negativa grande. Esto es una consecuencia de la respuesta rápida de los electrones de conducción a la excitación electromagnética externa. Este comportamiento cuasi-libre es como un apantallamiento que causa un amortiguamiento muy rápido de los campos electromagnéticos dentro del metal. 

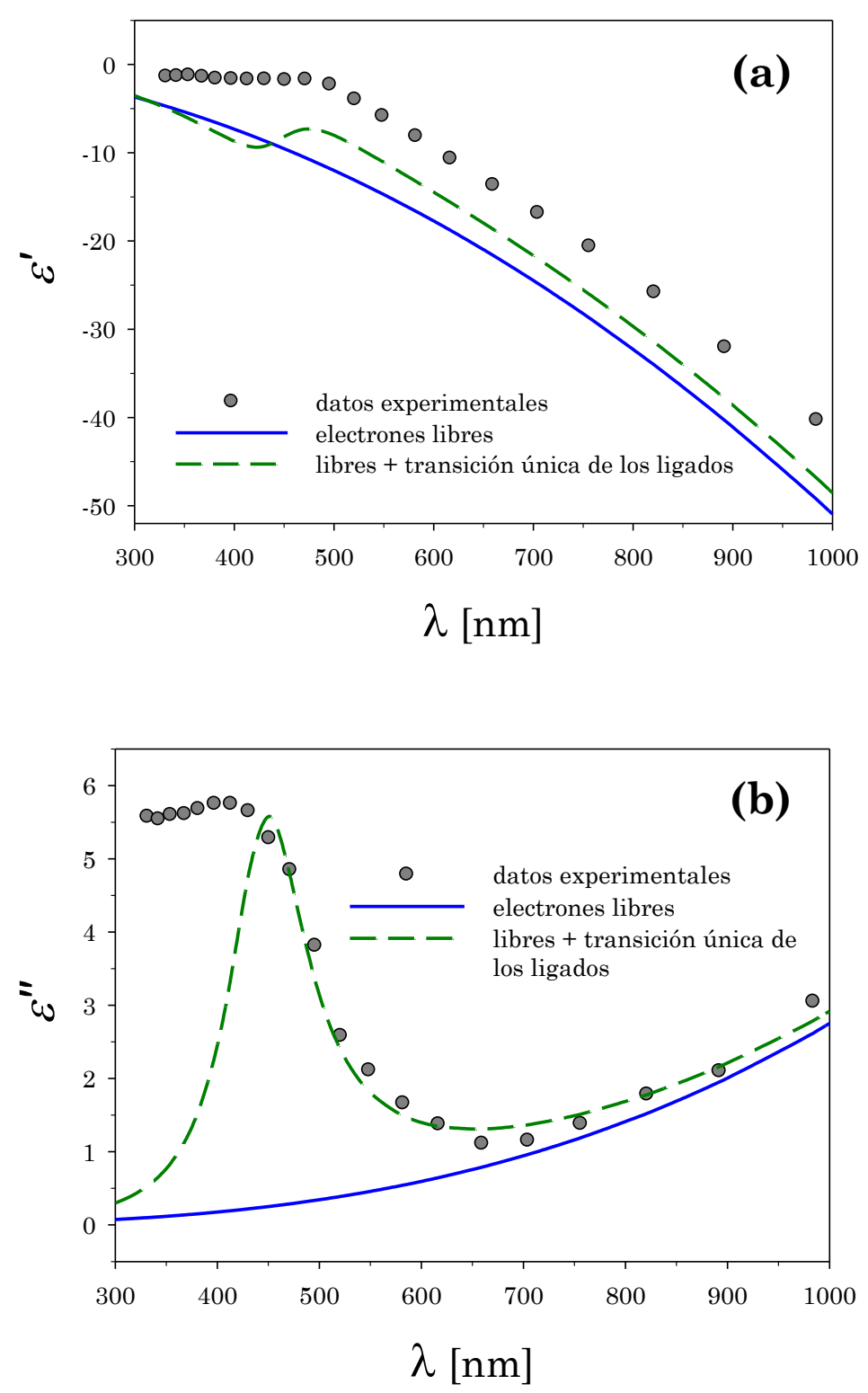

Figure 3.12: (a) Parte real y (b) parte imaginaria de la función dieléctrica del oro. Los círculos corresponden a la función dieléctrica bulk experimental de Johnson y Christy. La línea sólida es la contribución de los electrones libres para un tamaño de $a=100 \mathrm{~nm}$, y la línea discontinua representa la suma de la contribución de los electrones libres y la contribución de la transición única de los electrones ligados, para el mismo tamaño. Los valores utilizados son: $\omega_{0}=4,19 \times 10^{15} \mathrm{~Hz}, \omega_{p}^{\prime}=4,48 \times 10^{15} \mathrm{~Hz}$ y $\gamma_{\text {ligados }}=8,95 \times 10^{14} \mathrm{~Hz}$ [Novotny and Hecht 2007].

Con respecto a la parte imaginaria, para $\lambda<600 \mathrm{~nm}$, la suma de los electrones libres y el modelo del electrón ligado de transición única no 
reproduce los datos experimentales. Para este modelo, $\varepsilon^{\prime \prime}$ se aparta de los datos experimentales entre 300 y 450 nm. Así, con la introducción de una corrección de interbanda única (ecuación (3.32)), se encuentra un acuerdo con la curva experimental sólo para $450 \mathrm{~nm}<\lambda<1000 \mathrm{~nm}$.

Un comportamiento similar (en cuanto a la falta de ajuste) se observa para el caso de la plata y el cobre, tanto para las partes real como imaginaria. Por lo tanto, es necesario un modelo más completo que considere explícitamente otras transiciones interbanda para describir la expresión compleja de $\varepsilon(\omega, a)$.

\subsubsection{Contribución de los electrones ligados: transiciones múltiples}

Debido a que el modelo de la contribución de los electrones ligados de transición única falla para tamaños de partículas comparables al camino libre medio de los electrones de conducción, existe la necesidad de una descripción adecuada de las propiedades ópticas para grandes clusters formados por cientos de átomos. Este es un tema que se ha discutido desde hace más de cincuenta años [Kreibig and von Fragstein 1969, Kreibig 1970]. En esta Sección se muestra un formalismo teórico para evaluar la contribución de interbanda a la función dieléctrica. Esta contribución se basa en un análisis semi empírico.

Algunos autores [Pinchuk et al. 2004] han analizado la influencia de las transiciones electrónicas interbanda en la frecuencia, la amplitud y el ancho de banda de la resonancia del plasmón superficial en nanoclusters metálicos pequeños. Sus resultados teóricos de plata y oro, incluyendo las transiciones interbanda en el modelo, conducen a un valor más razonable para el ancho medio del plasmón. Una primera aproximación estuvo basada 
en el concepto de que la contribución debida a las transiciones interbanda permanece invariable cuando el material pasa de su tamaño macroscópico a pequeñas partículas. Luego, este hecho ha sido modificado, especialmente para los metales nobles, considerando las transiciones de los electrones ligados a los niveles de conducción con una frecuencia característica $\omega_{0}$.

Para la contribución de las transiciones de los electrones ligados a la función dieléctrica en sólidos reales, $\omega_{0}$ debe extenderse para cubrir todas las transiciones permitidas a los niveles de la banda de conducción, como se indica en la Figura 3.13.

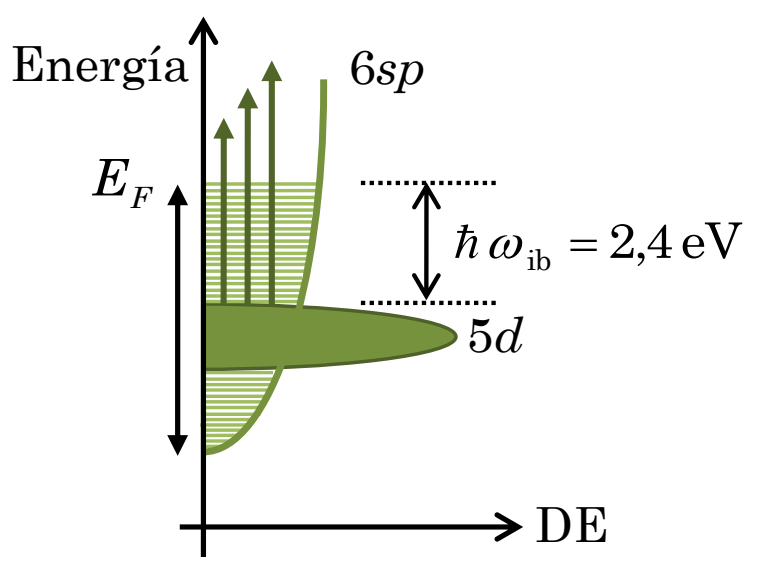

Figure 3.13: Diagrama esquemático de los niveles de energía del oro. Las transiciones interbanda desde el estado $d$ a los estados $s p$ están representadas por flechas verticales. DE: densidad de estados.

Si la densidad de estados en esta banda está representada por $D\left(\omega_{0}\right)$, la función dieléctrica relativa correspondiente a la contribución de los electrones ligados puede escribirse como:

$$
\varepsilon_{\text {ligados }}(\omega)=\int D\left(\omega_{0}\right)(1-F) \varepsilon_{\text {ligados }}^{L}\left(\omega, \omega_{0}\right) d \omega_{0}
$$


donde $F$ es la distribución de Fermi, $(1-F)$ es la probabilidad de que el estado superior no esté ocupado y $\varepsilon_{\text {ligados }}^{L}\left(\omega, \omega_{0}\right)$ es la función dieléctrica de Lorentz dada por la ecuación (3.32). Algunos autores [Rosei et al. 1973, Rosei 1974] han dado una forma explícita para $D\left(\omega_{0}\right)$ correspondiente a los metales nobles como el oro y la plata, y también muestran la forma de cómo calcular los límites de la integral de la ecuación (3.36). Por otra parte, Inouye y coautores [Inouye et al. 1998] han demostrado una expresión simplificada de este cálculo para el oro, asumiendo que la curvatura de la banda $d$ puede ser ignorada. En este caso, la función dieléctrica se puede escribir como:

$$
\mathcal{E}_{\text {ligados }}(\omega)=K_{\text {bulk }} \int_{\omega_{g}}^{\infty} \frac{\sqrt{x-\omega_{g}}}{x}[1-F(x, T)] \frac{\left(x^{2}-\omega^{2}+\gamma_{\text {ligados }}^{2}+i 2 \omega \gamma_{\text {ligados }}\right)}{\left(x^{2}-\omega^{2}+\gamma_{\text {ligados }}^{2}\right)^{2}+4 \omega^{2} \gamma_{\text {ligados }}^{2}} \mathrm{~d} x
$$

donde, $\hbar \omega_{g}$ es la energía de la banda prohibida $\left(E_{g}\right), F\left(x, E_{F}, T\right)$ es la función de distribución de la energía de Fermi del electrón de conducción de energía $\hbar x$ a la temperatura $T$ con energía de Fermi $E_{F} ; \gamma_{\text {ligados }}$ es la constante de amortiguamiento de la banda $d$ a la banda de transición sp y $K_{b u l k}$ es un factor de proporcionalidad. La referencia de la energía se supone en la parte superior de la banda $d$, como se indica en la Figura 3.13.

La Figura 3.14 muestra los cálculos de la contribución de los electrones libres (ecuaciones (3.29) y (3.30)) y la contribución de los electrones ligados de las transiciones múltiples (ecuación (3.37)) a la función dieléctrica del oro, considerando nuevamente un radio de $100 \mathrm{~nm}$ para representar un tamaño macroscópico. La suma de estas contribuciones (línea sólida) se compara con los datos experimentales (círculos) dados en la referencia [Johnson and Christy 1972] del metal macroscópico. Algunos parámetros se tomaron de la literatura, mientras que otros se calcularon 
previamente por los autores Scaffardi y Tocho [Scaffardi and Tocho 2006]. Estos parámetros tales como $K_{b u l k}, E_{g}, E_{F}$ y $\gamma_{\text {ligados }}$ producen efectos diferentes e independientes sobre la función dieléctrica.
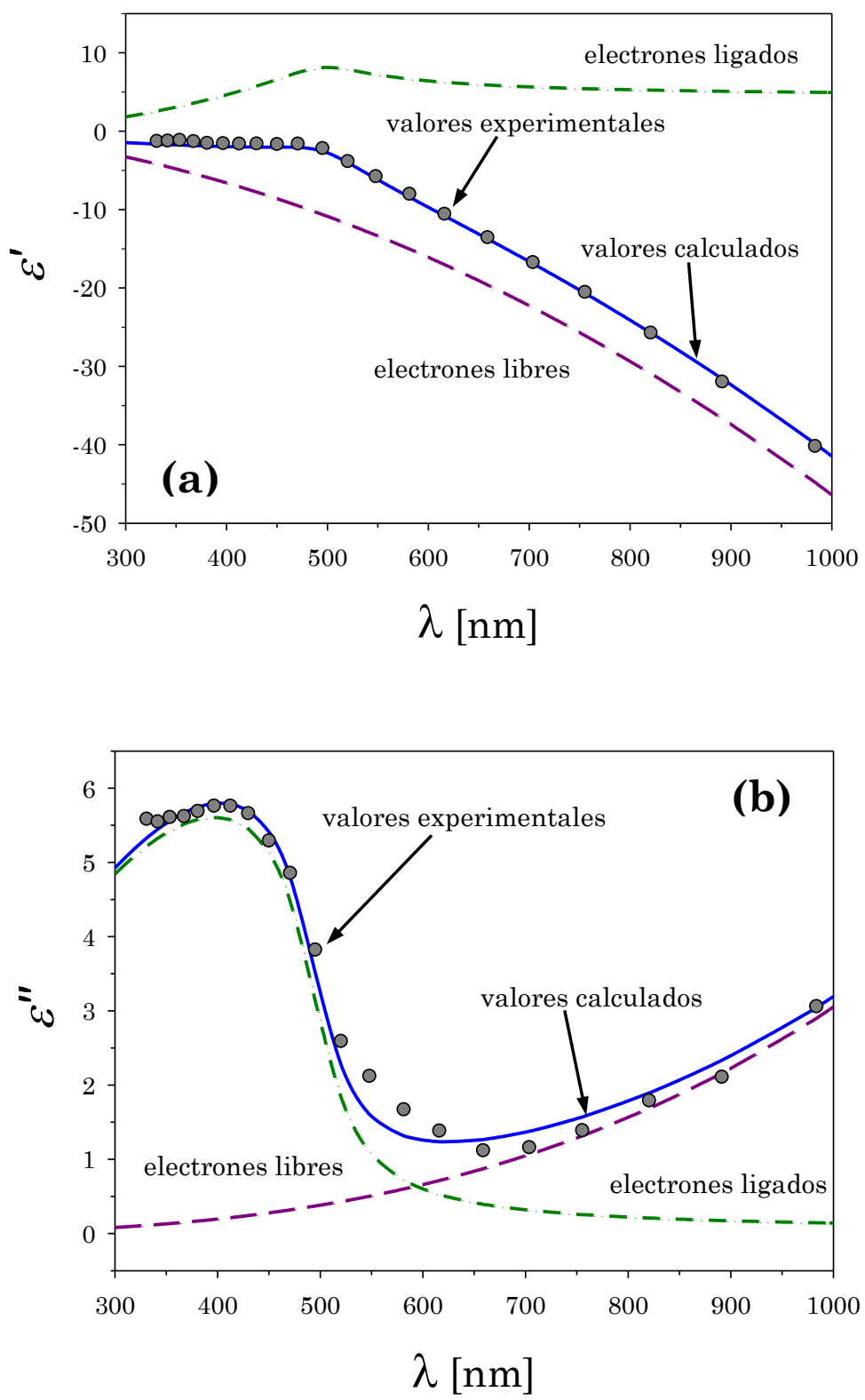

Figura 3.14: Cálculos de las contribuciones de los electrones libres y de los electrones ligados de las transiciones múltiples a la función dieléctrica bulk del oro. La suma de las contribuciones (línea sólida) es comparada con los datos experimentales del oro (círculos) [Johnson and Christy 1972]. 
Los parámetros de ajuste y otros valores relevantes para el oro macroscópico se resumen en la Tabla 3.1. Se puede observar que con estos parámetros las curvas muestran un excelente acuerdo con los datos experimentales sin necesidad de agregar una constante arbitraria.

Tabla 3.1: Parámetros del oro para contribuciones de libres y ligados

\begin{tabular}{|c|c|c|c|}
\hline Parámetros del Au & Símbolos & Valores & Referencias \\
\hline Frecuencia de plasma & $\omega_{p}$ & $13 \times 10^{15} \mathrm{~Hz}$ & $\begin{array}{l}\text { [Granqvist and } \\
\text { Hunderi 1977] }\end{array}$ \\
\hline $\begin{array}{l}\text { Constante de amortiguamiento } \\
\text { para los electrones libres }\end{array}$ & $\gamma_{\text {libres }}$ & $1,1 \times 10^{14} \mathrm{~Hz}$ & $\begin{array}{l}\text { [Johnson and } \\
\text { Christy 1972] }\end{array}$ \\
\hline $\begin{array}{l}\text { Coeficiente de la contribución } \\
\text { del electrón ligado }\end{array}$ & $K_{\text {bulk }}$ & $2,3 \times 10^{24}$ & $\begin{array}{l}\text { [Scaffardi and } \\
\text { Tocho 2006] }\end{array}$ \\
\hline Energía del gap & $E_{g}$ & $2,1 \mathrm{eV}$ & $\begin{array}{l}\text { [Scaffardi and } \\
\text { Tocho 2006] }\end{array}$ \\
\hline Energía de Fermi & $E_{F}$ & $2,5 \mathrm{eV}$ & $\begin{array}{l}\text { [Scaffardi and } \\
\text { Tocho 2006] }\end{array}$ \\
\hline $\begin{array}{l}\text { Constante de amortiguamiento } \\
\text { para los electrones ligados }\end{array}$ & $\gamma_{\text {ligados }}$ & $2,4 \times 10^{14} \mathrm{~Hz}$ & [Inouye et al. 1998] \\
\hline
\end{tabular}

\subsubsection{Corrección de los electrones ligados por tamaño}

Para la contribución de los electrones ligados, no se conoce ninguna expresión funcional que pueda cambiar los parámetros en la ecuación (3.37) y hacerla dependiente del tamaño de la partícula. Algunos autores [Logunov et al. 1997, Boyen et al. 2002, Scaffardi and Tocho 2006] introducen la idea de que la densidad electrónica de estados es diferente para las nanopartículas de distintos tamaños. Este hecho afecta a la expresión (3.37) donde una parte del integrando es la densidad de estados de la banda 
superior. Dado que las partículas pequeñas tienen mayor espaciamiento entre los estados electrónicos, es válido concluir que la densidad de estados será menor para nanopartículas muy pequeñas. Por lo tanto, para tener en cuenta este hecho se propuso [Scaffardi and Tocho 2006], cambiar el factor de proporcionalidad $K_{\text {bulk }}$ en la contribución de los electrones ligados a partir de su valor macroscópico aceptado por $K_{t}=K_{b u l k}\left(1-\exp \left(-a / a_{0}\right)\right)$, donde $a$ es el radio de la partícula y $a_{0}$ es un factor de escala que representa el tamaño para el cual la densidad de estados alcanza el 67\% del valor que correspondería al bulk.

Las Figuras 3.15 (a) y (b) muestran el comportamiento espectral de las partes real e imaginaria de las contribuciones de los electrones ligados del oro para diferentes tamaños nanométricos y subnanométricos, utilizando la ecuación (3.37) con la corrección $K_{t}=K_{\text {bulk }}\left(1-\exp \left(-a / a_{0}\right)\right)$.

Se puede observar que las curvas correspondientes a los tamaños más grandes que $2 \mathrm{~nm}$ se solapan unas con otras. Esto ocurre debido a que la corrección incluida en la expresión de $K_{t}$ se hace despreciable.

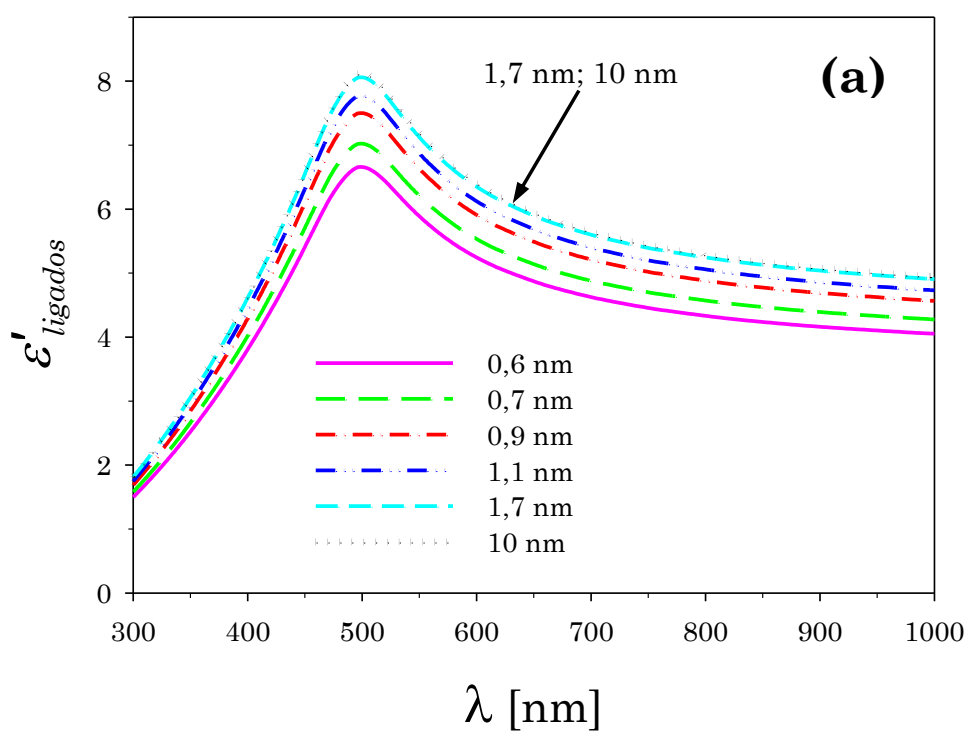




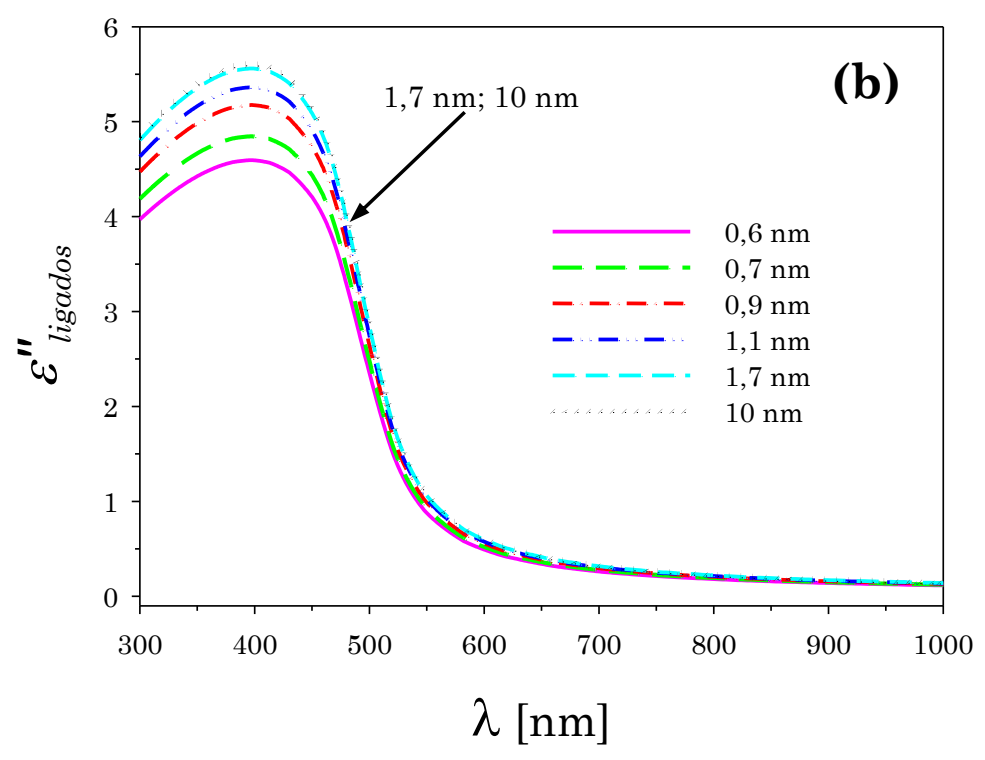

Figure 3.15: Contribución de los electrones ligados a la función dieléctrica del oro [Johnson and Christy 1972], incluyendo la corrección por tamaño. La corrección es despreciable para tamaños cercanos a $1,7 \mathrm{~nm}$.

Las diferentes modificaciones en las partes real e imaginaria de la función dieléctrica que se han descripto anteriormente (derivadas de la contribución de los electrones libres y ligados), se utilizarán en el Capítulo 6 para ajustar los resultados experimentales y de esta manera determinar la distribución de tamaños de partículas de las suspensiones coloidales. 


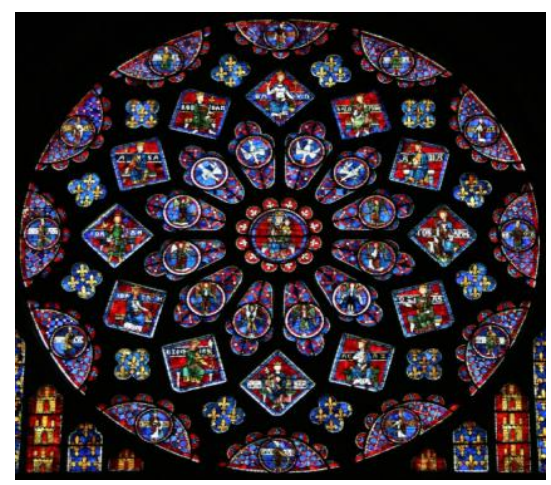

Catedral de Ntra. Sra. de Chartres Chartres, Francia

\section{Capítulo 4}

\section{Propagación de ondas electromagnéticas en interfases planas metal-dieléctrico}

La fundamentación matemática de las ondas superficiales de oscilación de electrones (plasmones superficiales) se estableció a comienzos del siglo XX bajo el contexto de ondas de radio que se propagaban a lo largo de la superficie de un conductor de conductividad finita [Sommerfeld 1899, Zenneck 1907].

Para el caso de luz visible, en 1968 Kretschmann y Raether [Kretschmann and Raether 1968] demostraron la posibilidad de excitar ondas superficiales utilizando un arreglo experimental basado en un prisma sobre el cual se depositó una delgada película metálica con un espesor de unas pocas decenas de nanómetros. El análisis de las características de este dispositivo permitió elaborar una descripción unificada de estos fenómenos introduciendo el concepto de "polaritones plasmones superficiales".

Se aborda en este Capítulo la teoría relacionada con el diseño de un sensor tipo Kretschmann, basado en el principio de resonancia plasmónica. 
Se presenta el método matricial para el cálculo de la reflectividad de una onda de luz monocromática con polarización $p$ y $s$ que interactúa en un sistema de multicapas.

\subsection{Polaritones plasmones superficiales en una interfase plana}

Los polaritones plasmones superficiales son excitaciones electromagnéticas propagantes en una interfase entre un conductor y un dieléctrico, evanescentemente confinadas en la dirección perpendicular hacia ambos medios. Estas ondas superficiales electromagnéticas surgen a partir del acoplamiento del campo electromagnético con las oscilaciones del plasma de electrones en el conductor.

Con el fin de entender las propiedades físicas de los polaritones plasmones superficiales, se comienza por considerar una interfase plana, donde el medio 1 está caracterizado por una función dieléctrica compleja dependiente de la frecuencia, $\varepsilon_{1}(\omega)$, mientras que el medio 2 posee una función dieléctrica $\varepsilon_{2}(\omega)$ que se supone real. La interfase coincide con el plano $\mathrm{Z}=0$ como se observa en la Figura 4.1. Con estas condiciones, el objetivo es encontrar soluciones homogéneas de las ecuaciones de Maxwell que estén localizadas en la interfase.

Las ecuaciones rotacionales de Maxwell combinadas con las relaciones constitutivas, considerando además campos armónicos, permiten escribir una ecuación de onda para la amplitud del campo eléctrico:

$$
\nabla(\nabla \cdot \mathbf{E}(\mathbf{r}, \omega))-\nabla^{2} \mathbf{E}(\mathbf{r}, \omega)-\frac{\omega^{2}}{\mathrm{c}^{2}} \varepsilon(\mathbf{r}, \omega) \mathbf{E}(\mathbf{r}, \omega)=0
$$


con $\varepsilon(\mathbf{r}, \omega)=\varepsilon_{1}(\omega)$ si $\mathrm{Z}<0 \quad$ y $\quad \varepsilon(\mathbf{r}, \omega)=\varepsilon_{2}(\omega)$ si $\mathrm{Z}>0$. Para que la localización de la onda se produzca efectivamente en la interfase, los campos electromagnéticos deben decaer exponencialmente con el aumento de la distancia desde la interfase hacia ambos semi-espacios.

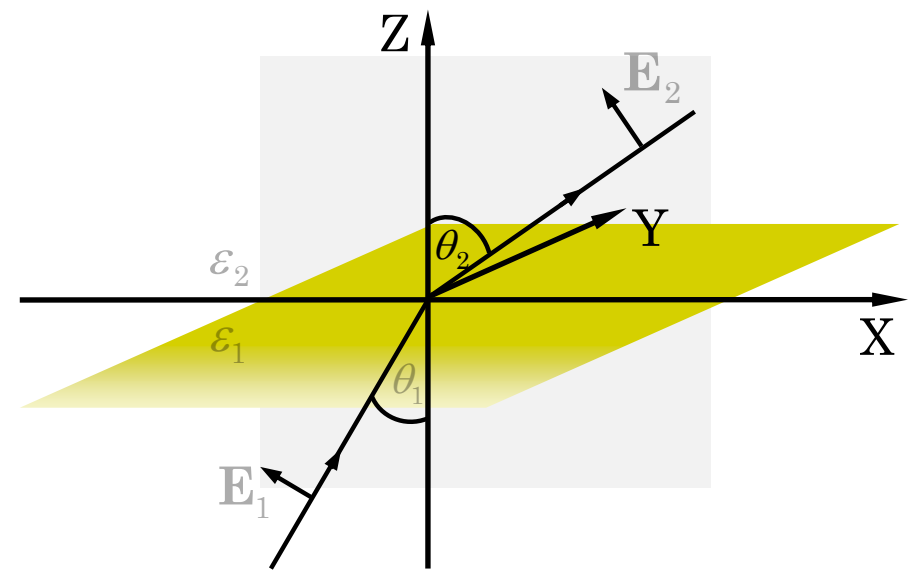

Figura 4.1: Interfase entre dos medios caracterizados por sus funciones dieléctricas $\varepsilon_{1}(\omega)$ y $\varepsilon_{2}(\omega)$, respectivamente. La interfase está definida por $\mathrm{Z}=0$ en un sistema de coordenadas cartesianas. En cada semi-espacio se considera una onda con polarización $p$.

Considerando que las ondas planas tienen polarización $p$ en ambos medios $j=1$ y $j=2$, y el vector de propagación está contenido en el plano $\mathrm{XZ}$, éstas pueden escribirse como:

$$
\mathbf{E}_{j}=\left(\begin{array}{c}
E_{j, x} \\
0 \\
E_{j, z}
\end{array}\right) e^{i \mathrm{k}_{x} x-i \omega t} e^{i \mathrm{k}_{j, z} z}, \quad \quad j=1,2
$$

De acuerdo a las condiciones de contorno en la frontera entre dos medios, el vector de onda paralelo a la interfase se conserva, por lo que se cumplen las siguientes relaciones para las componentes del vector de onda: 


$$
\mathrm{k}_{x}^{2}+\mathrm{k}_{j, z}^{2}=\varepsilon_{j} \mathrm{k}^{2}, \quad j=1,2
$$

Aquí $\mathrm{k}=2 \pi / \lambda$, donde $\lambda$ es la longitud de onda del vacío. Considerando que los campos de desplazamiento no tienen fuentes, es decir $\nabla \cdot \mathbf{D}=0$, se llega a:

$$
\mathrm{k}_{x} E_{j, x}+\mathrm{k}_{j, z} E_{j, z}=0, \quad j=1,2
$$

lo cual permite reescribir la ecuación (4.2) como:

$$
\mathbf{E}_{j}=E_{j, x}\left(\begin{array}{c}
1 \\
0 \\
-\mathrm{k}_{x} / \mathrm{k}_{j, z}
\end{array}\right) e^{i \mathrm{k}_{j, z} z} e^{i \mathrm{k}_{x} x-i \omega t}, \quad j=1,2
$$

Mientras que las expresiones (4.3) y (4.4) imponen condiciones que definen los campos en cada medio, se deben utilizar las condiciones de frontera para igualar estos campos en la interfase. Para ello se requiere la continuidad de la componente paralela de $\mathbf{E}$ y la componente perpendicular de $\mathbf{D}$, lo cual conduce a otro conjunto de ecuaciones:

$$
\begin{gathered}
E_{1, x}-E_{2, x}=0 \\
\varepsilon_{1} E_{1, z}-\varepsilon_{2} E_{2, z}=0
\end{gathered}
$$

Las expresiones (4.4) y (4.6) forman un sistema homogéneo de cuatro ecuaciones para las cuatro componentes desconocidas del campo. La existencia de una solución requiere que el respectivo determinante sea nulo. Esto ocurre para $\mathrm{k}_{x}=0$ (solución trivial, que no describe excitaciones que viajan a lo largo de la interfase), o para: 


$$
\varepsilon_{1} \mathrm{k}_{2, z}-\varepsilon_{2} \mathrm{k}_{1, z}=0
$$

Combinando esta expresión con la ecuación (4.3), se llega a la relación de dispersión, es decir, relación entre el vector de onda a lo largo de la dirección de propagación (eje X contenido en la interfase) y la frecuencia angular $\omega$ :

$$
\mathrm{k}_{x}^{2}=\frac{\varepsilon_{1} \varepsilon_{2}}{\varepsilon_{1}+\varepsilon_{2}} \mathrm{k}^{2}=\frac{\varepsilon_{1} \varepsilon_{2}}{\varepsilon_{1}+\varepsilon_{2}} \frac{\omega^{2}}{\mathrm{c}^{2}}
$$

Para la componente normal del vector de onda se obtiene:

$$
\mathrm{k}_{j, z}^{2}=\frac{\varepsilon_{j}^{2}}{\varepsilon_{1}+\varepsilon_{2}} \mathrm{k}^{2}, \quad j=1,2
$$

A partir de las expresiones (4.8) y (4.9), se pueden discutir las condiciones que se deben cumplir para que exista un modo en la interfase. Por sencillez se asume que las partes imaginarias de las funciones dieléctricas son pequeñas comparadas con las partes reales, para poder despreciarlas. Como el interés está centrado en ondas que se propagan a lo largo de la interfase, se requiere que $\mathrm{k}_{x}$ sea real, entonces en la ecuación (4.8) tanto el producto como la suma de las funciones dieléctricas deben ser ambas positivas o negativas. Además se necesita que las componentes normales del vector de onda sean puramente imaginarias en ambos medios, para dar lugar a las soluciones que decaen exponencialmente, lo cual se puede conseguir sólo si la suma del denominador de la ecuación (4.9) es negativa. De esto se concluye que las condiciones para que exista un modo en la interfase son:

$$
\varepsilon_{1}(\omega) \cdot \varepsilon_{2}(\omega)<0
$$




$$
\varepsilon_{1}(\omega)+\varepsilon_{2}(\omega)<0
$$

lo cual significa que una de las funciones dieléctricas debe ser negativa con un valor absoluto mayor que el de la otra. En el caso de algunos metales nobles como el oro y la plata, tienen una parte real negativa grande y una parte imaginaria pequeña en la función dieléctrica. Por lo tanto, se demuestra que existen modos localizados en una interfase metal-dieléctrico.

Para introducir las pérdidas asociadas al scattering del electrón, se considera que la función dieléctrica del metal es compleja, como se vio en el Capítulo 3. Por lo tanto el número de onda también es un complejo $\mathrm{k}_{x}=\mathrm{k}_{x}^{\prime}+i \mathrm{k}_{x}^{\prime \prime}$, donde la parte real $\left(\mathrm{k}_{x}^{\prime}\right)$ determina la longitud de onda de los polaritones plasmones superficiales, mientras que la parte imaginaria $\left(\mathrm{k}_{x}^{\prime \prime}\right)$ está relacionada con el amortiguamiento de los mismos a medida que se propagan a lo largo de la interfase. Las partes real e imaginaria de $\mathrm{k}_{x}$, se pueden determinar a partir de la ecuación (4.8) suponiendo que la parte imaginaria de $\varepsilon_{1}$ es mucho menor que su parte real:

$$
\begin{gathered}
\mathrm{k}_{x}^{\prime} \approx \sqrt{\frac{\varepsilon_{1}^{\prime} \varepsilon_{2}}{\varepsilon_{1}^{\prime}+\varepsilon_{2}}} \frac{\omega}{\mathrm{c}} \\
\mathrm{k}_{x}^{\prime \prime} \approx \sqrt{\frac{\varepsilon_{1}^{\prime} \varepsilon_{2}}{\varepsilon_{1}^{\prime}+\varepsilon_{2}}} \frac{\varepsilon_{1}^{\prime \prime} \varepsilon_{2}}{2 \varepsilon_{1}^{\prime}\left(\varepsilon_{1}^{\prime}+\varepsilon_{2}\right)} \frac{\omega}{\mathrm{c}}
\end{gathered}
$$

Entonces, la longitud de onda de los polaritones plasmones superficiales (SPP) es:

$$
\lambda_{\mathrm{SPP}}=\frac{2 \pi}{\mathrm{k}_{x}^{\prime}} \approx \sqrt{\frac{\varepsilon_{1}^{\prime}+\varepsilon_{2}}{\varepsilon_{1}^{\prime} \varepsilon_{2}}} \lambda
$$


donde $\lambda$ es la longitud de onda de la luz de excitación en el vacío, como se expresó anteriormente.

La longitud de propagación de los polaritones plasmones superficiales a lo largo de la interfase está determinada por $\mathrm{k}_{x}^{\prime \prime}$ la cual, de acuerdo a la expresión (4.2), es responsable de un amortiguamiento exponencial de la amplitud del campo eléctrico. La longitud de decaimiento $1 / e$ del campo eléctrico en la interfase es $1 / k_{x}^{\prime \prime}$ o $1 /\left(2 k_{x}^{\prime \prime}\right)$ para la intensidad. Como ejemplo, en el caso del oro, que tiene una función dieléctrica compleja dada por $\varepsilon_{1}=-11,6+i 1,2$ para $\lambda=633 \mathrm{~nm}$, se obtiene una longitud de decaimiento de la intensidad de los polaritones plasmones superficiales de $\sim 10 \mu \mathrm{m}$ [Novotny and Hetch 2007].

La longitud de decaimiento de los campos eléctricos de los polaritones plasmones superficiales fuera de la interfase puede determinarse de la ecuación (4.9) para el primer orden en $\left|\varepsilon_{1}^{\prime \prime}\right| /\left|\varepsilon_{1}^{\prime}\right|$ usando la función dieléctrica compleja $\varepsilon_{1}$ como:

$$
\begin{gathered}
\mathrm{k}_{1, z}=\frac{\omega}{\mathrm{c}} \sqrt{\frac{\varepsilon_{1}^{\prime 2}}{\varepsilon_{1}^{\prime}+\varepsilon_{2}}}\left[1+i \frac{\varepsilon_{1}^{\prime \prime}}{2 \varepsilon_{1}^{\prime}}\right] \\
\mathrm{k}_{2, z}=\frac{\omega}{\mathrm{c}} \sqrt{\frac{\varepsilon_{2}^{2}}{\varepsilon_{1}^{\prime}+\varepsilon_{2}}}\left[1-i \frac{\varepsilon_{1}^{\prime \prime}}{2\left(\varepsilon_{1}^{\prime}+\varepsilon_{2}\right)}\right]
\end{gathered}
$$

Considerando los mismos parámetros del oro, la longitud de decaimiento de los campos eléctricos en el metal y en el dieléctrico (aire para nuestro ejemplo) es de $28 \mathrm{~nm}$ y $328 \mathrm{~nm}$, respectivamente [Novotny and Hetch 2007]. 


\subsubsection{Excitación del polaritón plasmón superficial}

Con el objeto de excitar un polaritón plasmón superficial es necesario satisfacer las condiciones de conservación de la energía y de la cantidad de movimiento. Para ello se requiere analizar las relaciones de dispersión de las ondas superficiales, es decir, la relación entre energía en términos de la frecuencia angular $\omega$, y la cantidad de movimiento en términos del vector de onda en la dirección de propagación $\mathrm{k}_{x}$, dada por las ecuaciones (4.8) y (4.12).

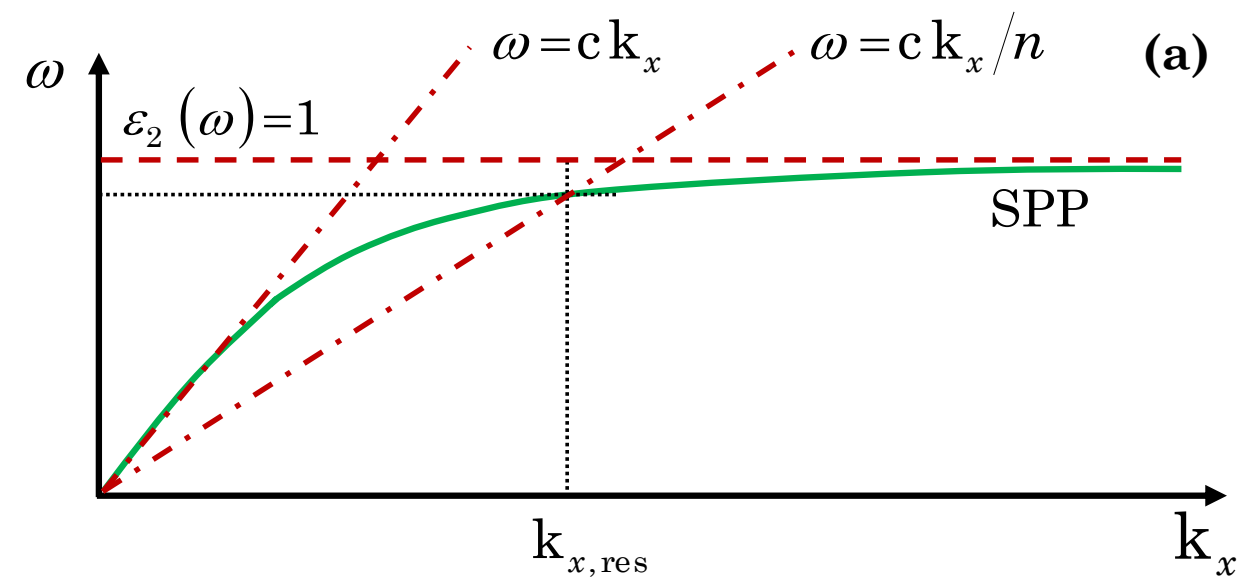

(b)
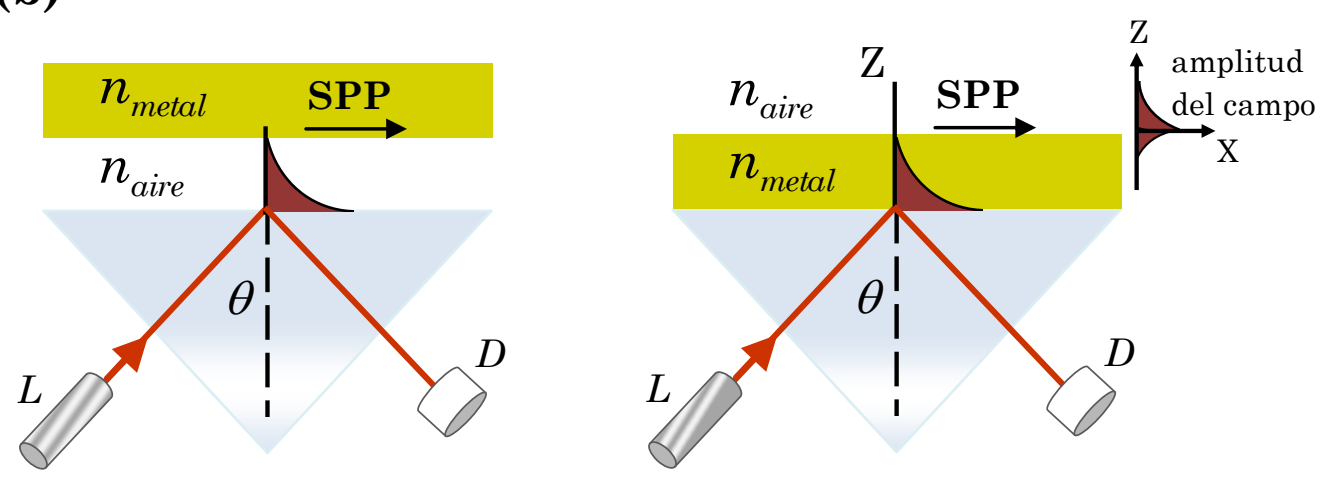

Figura 4.2: (a) Relación de dispersión de los polaritones plasmones superficiales para el caso particular de una interfase oro-aire, (b) arreglo experimental de la configuración de Otto (izquierda) y de Kretschmann (derecha); $L$ : láser y $D$ : detector. 
En la Figura 4.2 (a) se muestra la relación de dispersión de polaritones plasmones superficiales para el caso particular de una interfase oro-aire. La línea sólida representa la relación de dispersión que resulta de una función dieléctrica con una transición interbanda única. Las líneas rectas raya-punto corresponden a ondas planas libres que viajan en aire y en vidrio.

En la Figura 4.2 (b) se observa un esquema del arreglo experimental necesario para llevar a cabo la condición mostrada en la parte (a): en el panel de la izquierda se puede ver la llamada configuración de Otto en la que un pequeño espacio de aire entre el prisma de vidrio y la capa metálica permite acoplar el polaritón plasmón superficial en la interfase metal-aire. En el panel de la derecha se muestra la configuración de Kretschmann en la que la capa metálica se deposita directamente sobre el prisma. Si el espesor de esta capa metálica es suficientemente pequeño, la onda evanescente en el mismo logra acoplar el polaritón plasmón superficial en la interfase metalaire superior.

\subsection{Sistema de multicapas}

Cuando una onda de luz monocromática con polarización $p$ incide sobre una interfase metal-dieléctrico da lugar a una excitación de polaritones plasmones superficiales que se propagan a lo largo de la interfase, causando un mínimo en la intensidad de la luz reflejada por el metal. En este caso, la existencia del acoplamiento de estos modos superficiales puede ser comprendida a través del análisis de la reflectividad.

En un sistema de multicapas, se emplea un método matricial para el cálculo de la reflectividad que permite un tratamiento sistemático de cada capa y resulta fácil de extender al caso de una multicapa (Figura 4.3). 


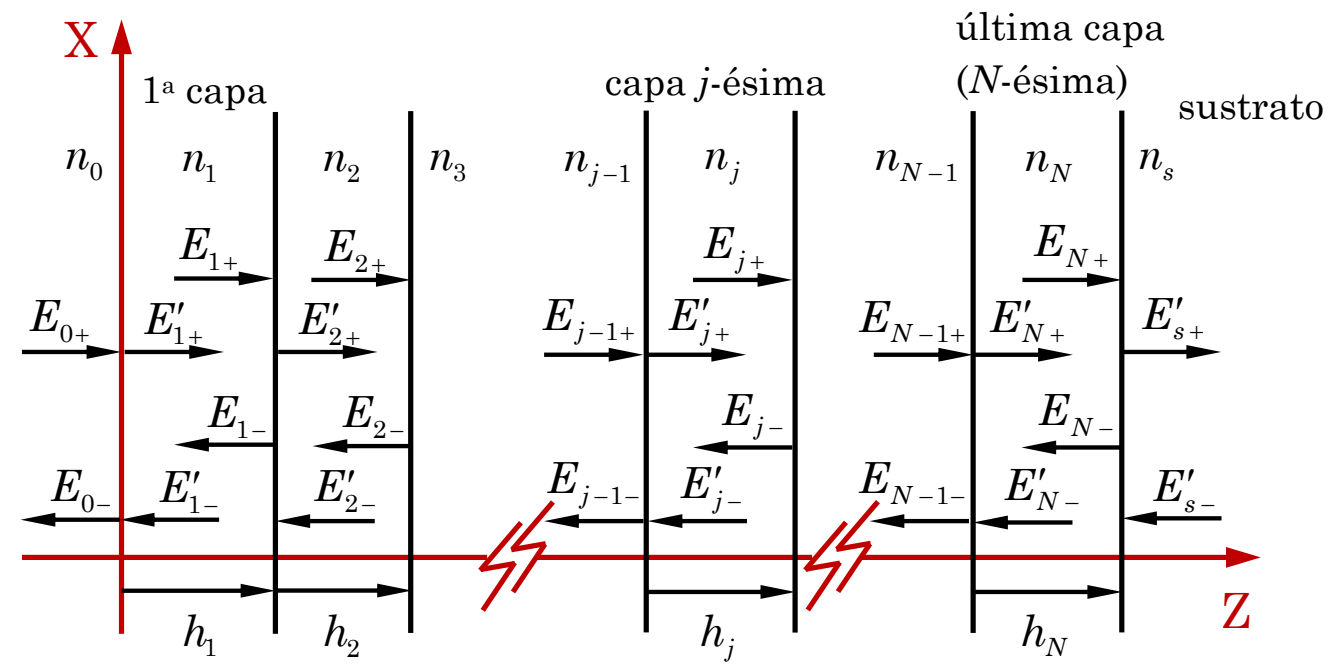

Figura 4.3: Representación esquemática de un sistema de multicapas. Cada capa está caracterizada por su índice de refracción $(n)$ y $h$ es el espesor de cada capa.

Aplicando las condiciones de continuidad en la frontera para el campo eléctrico en el sistema de multicapas (Figura 4.3), se obtiene:

$$
\left[\begin{array}{l}
E_{0+} \\
E_{0-}
\end{array}\right]=\mathcal{M}_{m}\left[\begin{array}{l}
E_{s+}^{\prime} \\
E_{s-}^{\prime}
\end{array}\right]
$$

donde $\mathcal{M}_{m}$ es la matriz característica de la multicapa, que viene dada por:

$$
\mathcal{M}_{m}=\left[\begin{array}{ll}
\mathcal{M}_{11} & \mathcal{M}_{12} \\
\mathcal{M}_{21} & \mathcal{M}_{22}
\end{array}\right]=\mathscr{D}_{0}^{-1}\left(\prod_{j=1}^{N} \mathcal{D}_{j} \boldsymbol{P}_{j} \mathscr{D}_{j}^{-1}\right) \mathscr{D}_{s}
$$

aquí $j$ indica el número de capas del sistema; $\mathscr{D}_{j}$ es la matriz de transmisión de la interfase de las capas y $\boldsymbol{P}_{j}$ es la matriz de propagación dentro de las capas. 
Las matrices de la interfase de las capas para polarización de onda $p$ y $s$ se definen como:

$$
\mathcal{D}_{j}^{p}=\left[\begin{array}{cc}
\cos \theta_{j} & -\cos \theta_{j} \\
n_{j} & n_{j}
\end{array}\right], \quad \mathcal{D}_{j}^{s}=\left[\begin{array}{cc}
1 & 1 \\
-n_{j} \cos \theta_{j} & n_{j} \cos \theta_{j}
\end{array}\right]
$$

donde $\theta_{j}$ es el ángulo de incidencia para la j-ésima capa y $n_{j}$ denota el j-ésimo índice de refracción de la capa.

La matriz de propagación $\boldsymbol{P}_{j}$ dentro de las capas puede ser escrita como:

$$
\boldsymbol{P}_{j}=\left[\begin{array}{cc}
e^{-i \phi_{j}} & 0 \\
0 & e^{i \phi_{j}}
\end{array}\right]
$$

$\operatorname{con} \phi_{j}=\frac{4 \pi n_{j} h_{j} \cos \theta_{j}}{\lambda}$ siendo $\lambda$ la longitud de onda incidente y $h_{j}$ el espesor de la j-ésima capa.

A partir de la ecuación matricial (4.17) puede obtenerse el coeficiente de reflexión de la multicapa para ambas polarizaciones ( $p$ y $s$ ) como:

$$
r=\frac{E_{0-}}{E_{0+}}=\frac{\mathcal{M}_{21}}{\mathcal{M}_{11}}
$$

Finalmente, la reflectividad de la multicapa se puede calcular con el coeficiente de reflexión de la ecuación (4.21):

$$
R=r r^{*}
$$




\subsection{Configuración de Kretschmann}

La excitación de las ondas superficiales con luz visible utilizando un prisma de acoplamiento fue obtenida por primera vez en 1968 por Kretschmann y Raether [Kretschmann and Raether 1968]. Sin embargo la demanda de sensores empleados tanto en la industria como en la ciencia, impone la necesidad de continuar investigando esta técnica.

Este tipo de configuración simple consiste en una o más capas metálicas delgadas depositadas sobre un prisma (Figura 4.4) y se basa en el principio de reflexión total interna.

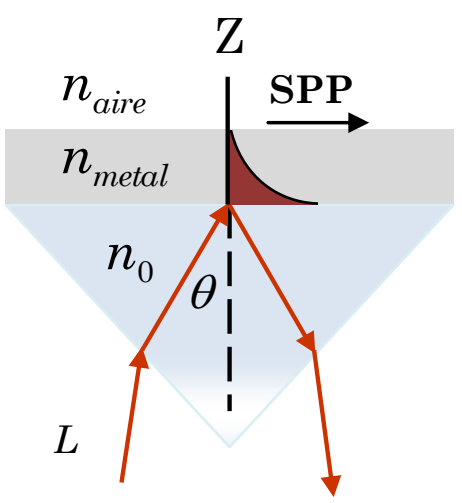

Figura 4.4: Arreglo experimental de la configuración de Kretschmann. L indica un láser, SPP el polaritón plasmón superficial y $\theta$ el ángulo de incidencia.

Cuando se hace incidir un haz de luz monocromática sobre la película metálica desde el prisma, la condición de resonancia para la excitación de los polaritones plasmones superficiales se produce a un cierto ángulo $(\theta)$ mayor que el ángulo crítico para reflexión total interna del haz dentro del prisma. Por lo tanto, la excitación de estos modos plasmónicos se observará como un mínimo en la luz reflejada (Figura 4.5). Como se observa en la 
Figura 4.5 el mínimo en la reflectividad depende del espesor de la capa metálica y del ángulo de incidencia.

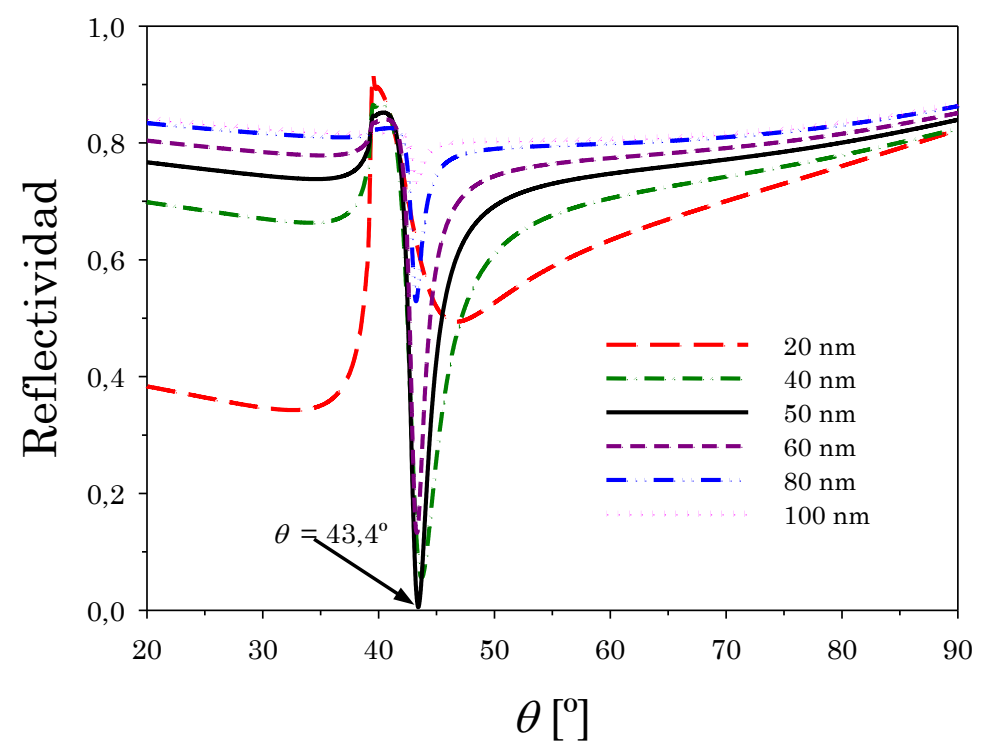

Figura 4.5: Reflectividad del haz de excitación en función del ángulo de incidencia para distintos espesores de oro. La longitud de onda del haz incidente es $632,8 \mathrm{~nm}$ y el índice de refracción complejo del oro a esta longitud de onda es $N=0,2+i 3,31$ ([Shulz 1954], [Shulz and Tangherlini 1954]).

La condición de resonancia asociada a la excitación de los polaritones plasmones superficiales tiene aplicación en varios sensores. Por ejemplo, la posición del mínimo en las curvas de reflectividad puede usarse como un indicador en los cambios del medio. Con este método, la adsorción o eliminación de diferentes materiales en la superficie metálica pueden detectarse con una precisión del orden de una submonocapa. En la Figura 4.6 se muestra el efecto de una capa de $5 \mathrm{~nm}$ de agua sobre una película de oro de $50 \mathrm{~nm}$ en vidrio, observándose un desplazamiento en la curva de resonancia. Si el ángulo de incidencia del haz de excitación se ajusta al mínimo en la curva de reflectividad, el depósito de una cantidad diminuta de material aumenta la señal de $\mathcal{R}$ drásticamente. Esto significa que el rango 
dinámico completo de una medida de la intensidad de ruido mínimo puede usarse para medir el espesor en un rango entre 0 y $5 \mathrm{~nm}$.

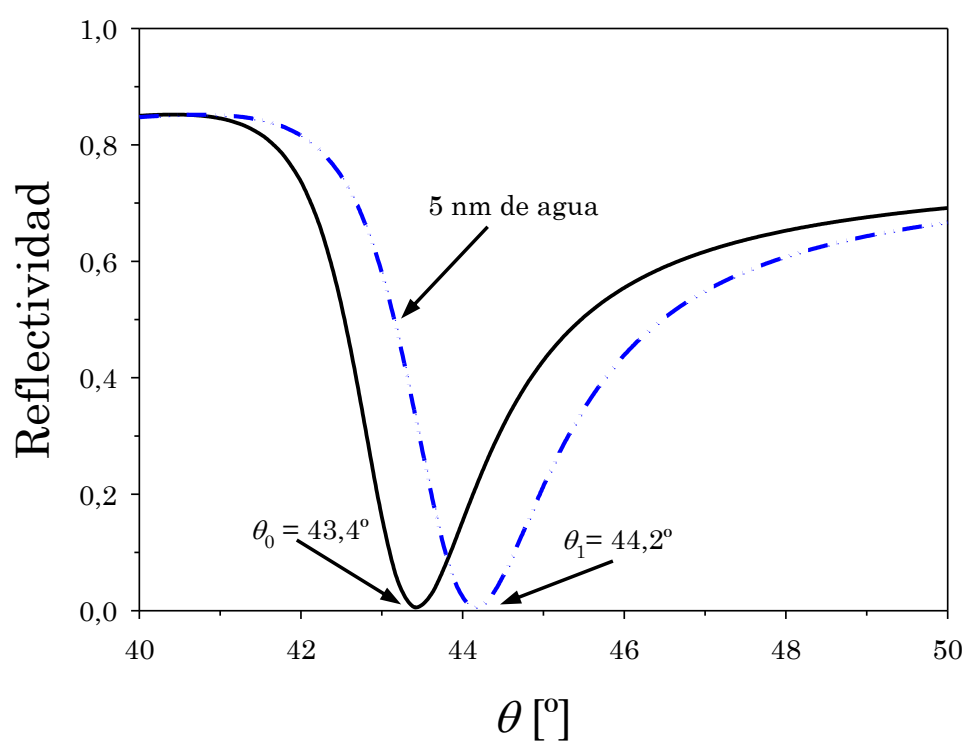

Figura 4.6: Polaritones plasmones superficiales usados en aplicaciones de sensores. Se observa el desplazamiento calculado de la curva de resonancia (curva negra) inducida por una capa de $5 \mathrm{~nm}$ de agua $(n=1,33)$ adsorbida en una película de oro de $50 \mathrm{~nm}$.

La razón de la extrema sensibilidad se debe a que la intensidad de la luz cerca de la superficie metálica está fuertemente reforzada. Como consecuencia, estos sensores son muy atractivos para aplicaciones en ensayos biológicos [Liedberg et al. 1983, Homola et al. 1999]. 


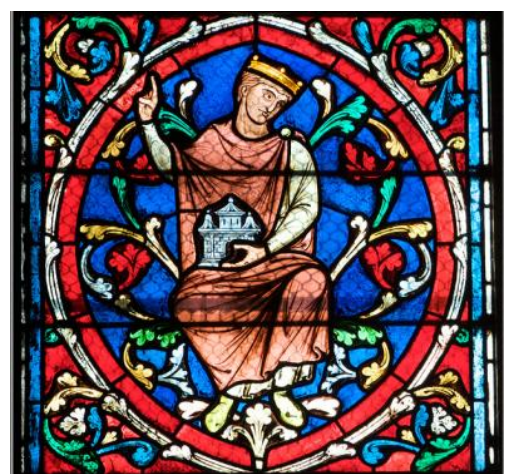

Catedral de Notre Dame París, Francia

\section{Capítulo 5}

\section{Generación de nanopartículas metálicas por ablación láser. Análisis por técnicas espectroscópicas}

La generación de nanopartículas metálicas (como así también de otros materiales y estructuras) se desarrolla desde hace varios años según dos estrategias: inicio por síntesis química de precursores seguida de autoensamblado de los productos para formar estructuras ordenadas de determinada simetría ("bottom-up") o inicio con una pieza macroscópica de material para formar la nanoestructura por actuación de un agente externo que produce maquinado o grabado (“top-down”).

Dentro de la primera estrategia se encuentran los métodos de química húmeda, en los que una sal metálica es reducida, obteniéndose la agregación de las nanopartículas metálicas para formar una suspensión. Si bien este método tiene la capacidad de producir nanopartículas de tamaños menores a $10 \mathrm{~nm}$ y con alto grado de monodispersión, el mismo produce compuestos no deseados (contaminantes) en la suspensión final y que muchas veces son difíciles de extraer sin perjudicar la propia suspensión de nanopartículas. 
Otro método dentro de esta estrategia es la formación de nanopartículas en fase gaseosa en forma de vapores metálicos supersaturados que condensan en nanopartículas al depositarse sobre una superficie de determinado sustrato o codepositarse con vapores atómicos de otro material. Si bien este método de síntesis es uno de los más flexibles (por permitir la generación de casi cualquier material con buena selección de masa de nanopartículas), conlleva la dificultad de la preparación de la fuente generadora de los vapores metálicos con infraestructura electrónica de control fino de temperaturas, cámara de alto vacío y fuente de haz molecular epitaxial.

Dentro de la estrategia "top-down", los métodos tradicionales litográficos son con haz de electrones (Electron Beam Lithography, EBL) y con haz de iones enfocados (Focused Ion Beam, FIB). En EBL, el material se cubre con una delgada película de polímero (comúnmente polimetilmetacrilato, PMMA), que se expone al haz de electrones escribiendo determinado patrón. Luego se expone la resina a un baño químico que disuelve las áreas no expuestas. La resina así tratada se utiliza como máscara para depositar las islas metálicas diseñadas sobre un sustrato. En FIB, se quitan las partes no deseadas de la capa metálica fresando las mismas con un haz de iones de Ga de $30 \mathrm{kV}$ de energía. Si bien los haces de ambas técnicas pueden enfocarse en spots de $5 \mathrm{~nm}$ y tienen una resolución del orden de $20 \mathrm{~nm}$, solo pueden procesar áreas muy pequeñas (del orden de unos pocos micrómetros), además de necesitar una cuidada preparación de la muestra.

En la última década se ha desarrollado una técnica física del tipo "top-down" basada en la ablación de blancos metálicos en líquidos con láseres pulsados de alta potencia, que reconoce sus orígenes en la técnica de deposición de películas delgadas asistida por láseres pulsados (Pulsed Laser Deposition, PLD). Mafuné y colaboradores [Mafuné et al. 2000a, Mafuné et al. 2000b, Mafuné et al. 2002a, Mafuné et al. 2002b, Mafuné et al. 2003] han 
estudiado la fabricación, las características, la estabilidad y el control de tamaño de nanopartículas de plata y oro generadas por ablación con láser de Nd:YAG (10 ns ancho de pulso) en solución acuosa con surfactantes. También se han generado nanopartículas metálicas de $\mathrm{Ti}$, Si y $\mathrm{Cu}$ con la misma técnica utilizando diferentes tipos de láseres pulsados entre $10 \mathrm{y}$ $20 \mathrm{~ns}$ de ancho de pulso [Dolgaev et al. 2002, Kazakevich et al. 2006]. En general, la ablación láser con estos anchos de pulsos tiende a producir partículas esféricas de varias decenas de nanómetros o de formas no esféricas.

Varios autores [Barcikowski et al. 2007a, Barcikowski et al. 2007b, Kabashin and Meunier 2007] han trabajado en la generación de nanopartículas metálicas por ablación láser con pulsos ultracortos (típicamente de 100 fs de ancho de pulso) que tienden a formar nanopartículas más pequeñas (pocas decenas de nanómetros) y, a diferencia de los métodos químicos que generalmente utilizan surfactantes, producen suspensiones limpias. La ablación láser pulsada también ha demostrado ser un método bastante eficaz para efectuar el proceso de fabricación de nanopartículas metálicas en dos etapas: primero generación y luego fragmentación [Besner et al. 2007], obteniendo en ambos casos nanopartículas esféricas.

En este trabajo de Tesis se pondrá en evidencia que las nanopartículas metálicas pueden oxidarse en presencia de medios líquidos, formando por lo general nanopartículas de estructura núcleo-recubrimiento [Schinca et al. 2009], cuyos radios de núcleo y espesores del recubrimiento deben ser determinados para su funcionalización en vista de futuras aplicaciones. La coloración observada de las suspensiones coloidales fabricadas por métodos físicos o químicos, está relacionada tanto al tamaño, la geometría, la estructura, el tipo de metal, la energía y el medio circundante de las partículas, como a su resonancia plasmónica. 
En este Capítulo se muestra el arreglo experimental para la fabricación de nanopartículas de cobre y plata mediante ablación láser de pulsos ultracortos, en diferentes medios líquidos. Luego se analizan las suspensiones coloidales a través de los espectros de extinción experimentales obtenidos con un espectrofotómetro. Estos resultados se complementan con análisis de microscopía AFM, HRTEM y espectroscopía Raman.

\subsection{Fabricación de nanopartículas por ablación láser de pulsos ultracortos}

El esquema experimental utilizado en esta Tesis para generar nanopartículas por ablación láser sobre un blanco sólido se muestra en la Figura 5.1.

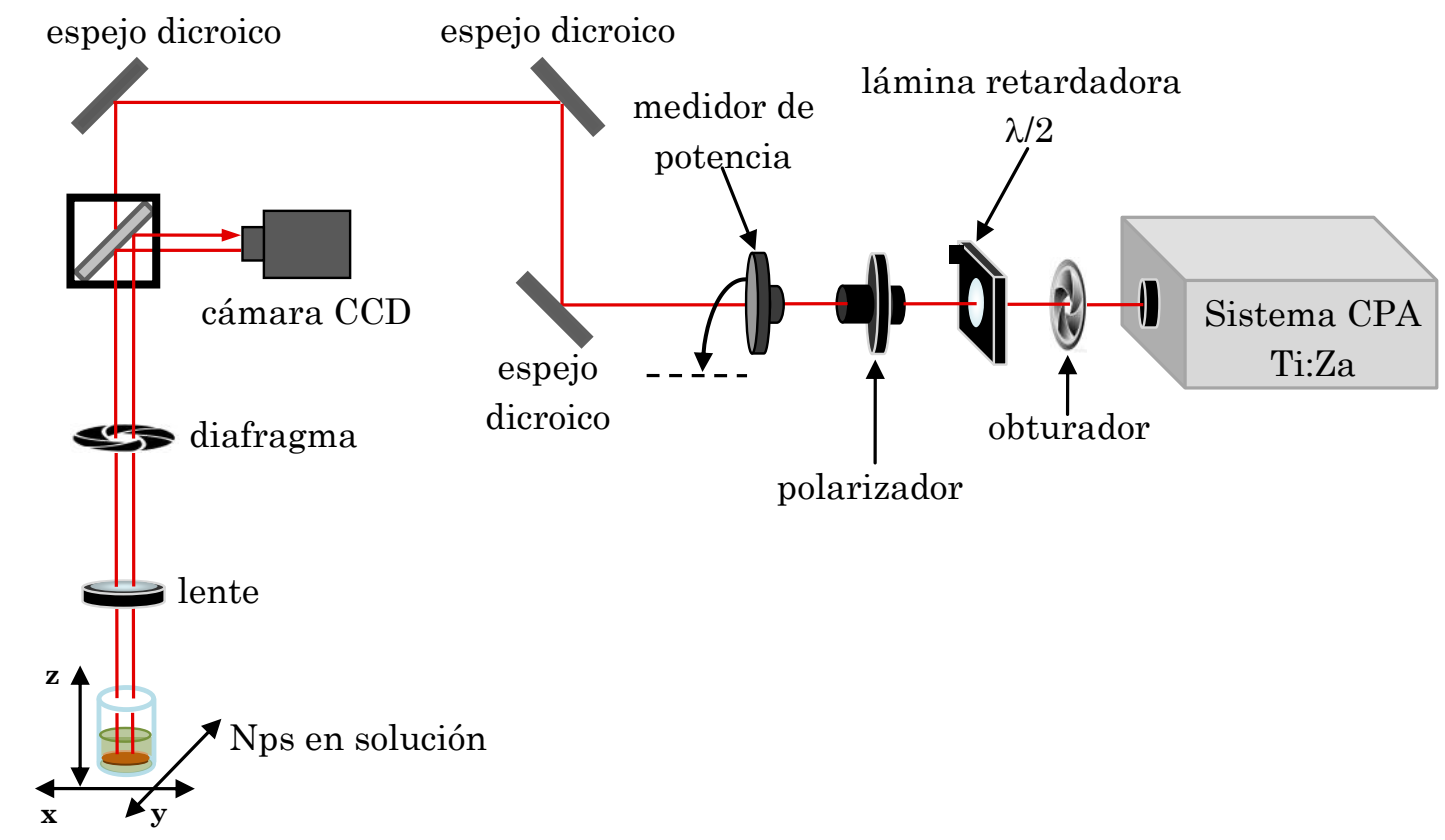

Figura 5.1: Arreglo experimental utilizado para la fabricación de las suspensiones coloidales de $\mathrm{Cu}$ y Ag con un láser de pulsos ultracortos de Ti:Za. 
El sistema amplificador de pulsos de un láser de Ti:Za emite pulsos de $100 \mathrm{fs}$ de ancho con una tasa de repetición de $1 \mathrm{kHz}$ centrada en una longitud de onda de $800 \mathrm{~nm}$, con una energía máxima de salida de $1 \mathrm{~mJ}$ por pulso. Para controlar la energía del haz se utilizó un sistema compuesto por un polarizador y una lámina retardadora de media longitud de onda (Figura 5.2 (a), panel derecho). La fabricación se realizó guiando el haz con un banco de maquinado como se observa en el panel izquierdo de la Figura 5.2 (a). El enfoque del haz del láser se logró con una lente de $5 \mathrm{~cm}$ de distancia focal sobre un disco $(\mathrm{Cu}$ o $\mathrm{Ag})$ de $1 \mathrm{~cm}$ de diámetro y $0,2 \mathrm{~cm}$ de espesor, que fue colocado sobre una plataforma de traslación X-Y-Z con una resolución de paso de $0,5 \mu \mathrm{m}$.
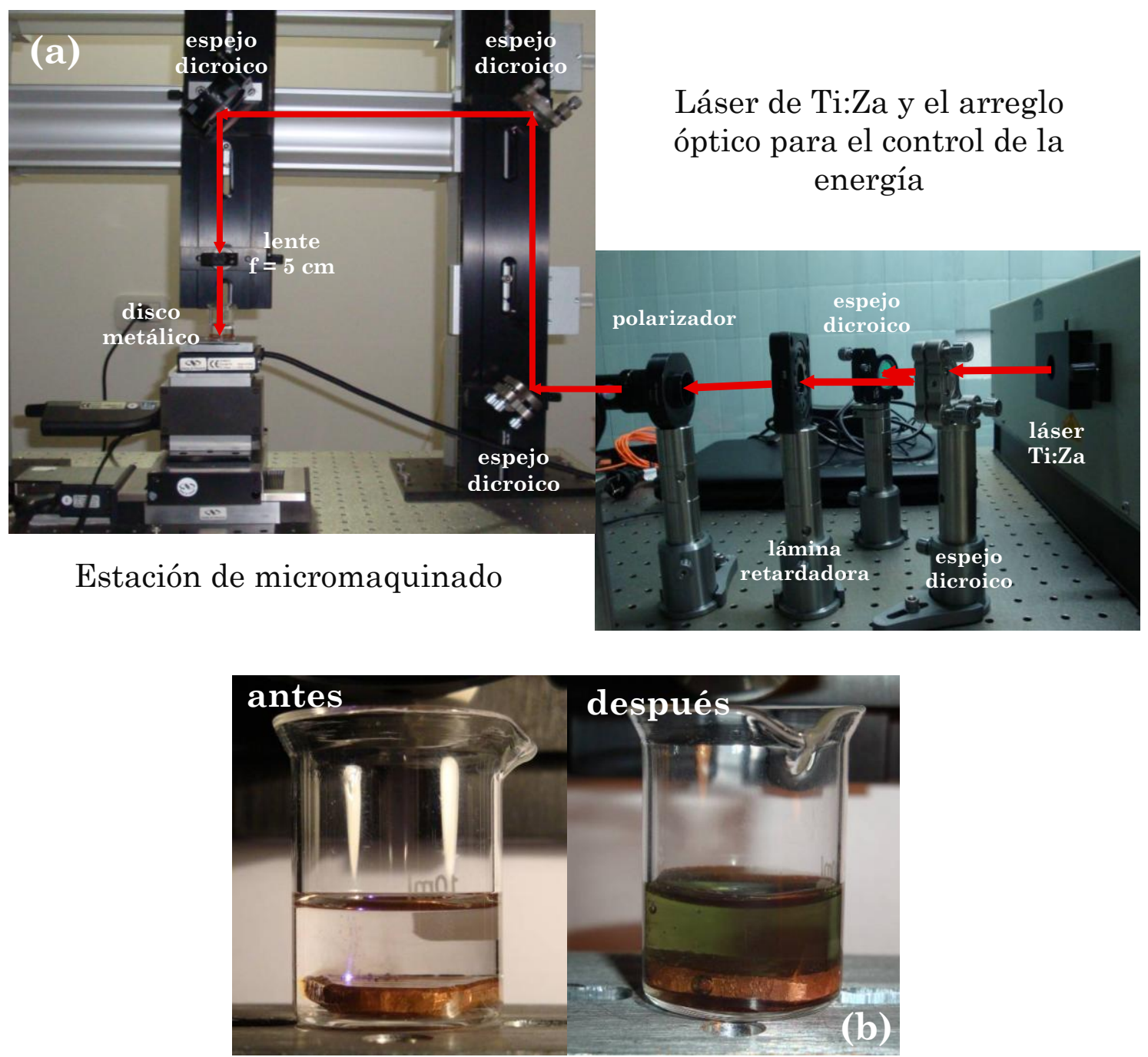

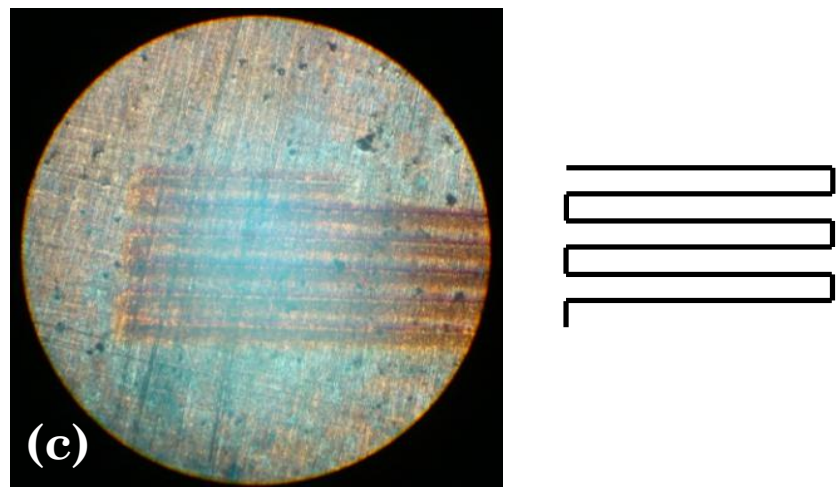

Figura 5.2: Ablación láser de pulsos ultracortos de Ti:Za. (a) Arreglo experimental para la fabricación de nanopartículas metálicas. En el panel derecho se observa el láser de Ti:Za y el arreglo óptico para el control de la energía, mientras que en el panel de la izquierda se muestra la estación de micromaquinado utilizada, con una vista parcial de los desplazadores X-Y-Z. (b) Blanco sólido de cobre en agua antes (panel izquierdo) y después (panel derecho) de la ablación. (c) Trazado lineal continuo que se repite consecutivamente a un cierto espaciado (meandros) ablacionado en el disco metálico.

Luego de haberse fijado cuidadosamente la posición de la muestra en el eje Z para enfocar el haz sobre la superficie del disco, como se muestra en el panel izquierdo de la Figura 5.2 (b), la misma se desplazó en el plano XY realizando un trazado lineal continuo que se repite consecutivamente a un cierto espaciado (meandros), para ablacionar siempre sobre la superficie original. Se obtuvieron segmentos de ablación en un sector de la superficie del disco metálico utilizando una velocidad de traslación de $0,1 \mathrm{~mm} / \mathrm{s}$ (Figura 5.2 (c)). Después de unos segundos de comenzado el proceso de ablación, el líquido presentaba una coloración típica de acuerdo al metal ablacionado y al medio en el que estaba sumergido (Figura 5.2 (b)).

El proceso de fabricación duró 8 minutos aproximadamente, generando suspensiones coloidales de diferentes colores típicos (Figura 5.3), debido a la presencia de un número importante de partículas de cobre o plata en solución. En el caso del cobre en agua, a medida que la energía va aumentando en el rango entre 50 y $500 \mu \mathrm{J}$, la coloración de la suspensión 
coloidal cambia de un verde pálido a un verde más intenso, como se puede ver en el panel izquierdo de la Figura 5.3 (a).
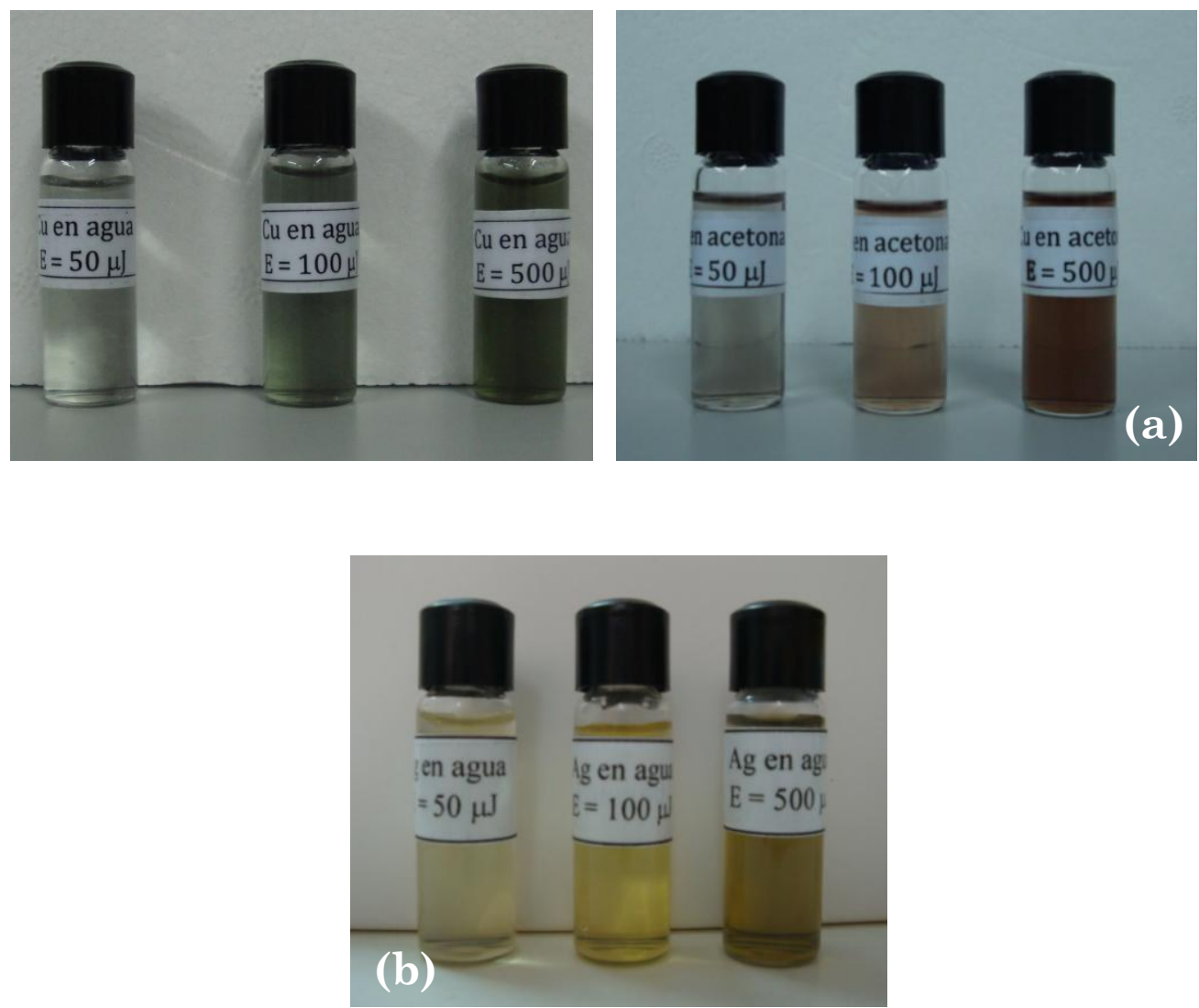

Figura 5.3: (a) Suspensiones coloidales de cobre generadas por ablación láser. En el panel izquierdo se muestran las suspensiones coloidales de cobre en agua a diferentes energías: $50 \mu \mathrm{J}, 100 \mu \mathrm{J}$ y $500 \mu \mathrm{J}$. En el panel de la derecha se observan suspensiones coloidales de cobre en acetona fabricadas a las mismas energías. (b) Suspensiones coloidales de plata en agua a diferentes energías: $50 \mu \mathrm{J}, 100 \mu \mathrm{J}$ y $500 \mu \mathrm{J}$.

En el caso del cobre en acetona, la coloración de la suspensión coloidal cambia desde un rojo pálido hasta un rojo intenso, en función de la energía utilizada, como se observa en el panel derecho de la Figura 5.3 (a). 
En la Figura 5.3 (b) se muestra la suspensión coloidal para el caso de plata en agua donde la coloración cambia desde un amarillo pálido hasta un amarillo intenso, a medida que la energía aumenta.

Para caracterizar el tamaño, la estructura y la configuración de las suspensiones coloidales de cobre o plata en agua y acetona, se realizaron mediciones de extinción óptica en el rango óptico extendido de 200 a $1100 \mathrm{~nm}$ utilizando un espectrofotómetro comercial de doble haz de marca Shimadzu.

\subsection{Espectroscopía de extinción óptica}

Los métodos tradicionales para determinación de tamaños de nanopartículas se basan en microscopía óptica o electrónica, dependiendo del tamaño. Estos métodos tienen, en muchos casos, la desventaja de necesitar de una preparación especial de la muestra y/o además presentar una estadística de muestreo deficiente. La técnica de espectroscopía óptica es más apropiada ya que las mediciones se pueden realizar sin tratamiento especial de la muestra, la adquisición de datos puede ser muy rápida, el análisis puede ser implementado in situ y es un excelente complemento a la microscopía electrónica.

La técnica de extinción espectral puede ser fácilmente implementada usando un espectrofotómetro comercial. Es muy simple y adecuada para determinar el tamaño de partículas en el rango de los micrones y submicrones cuando de partículas dieléctricas se trata [Scaffardi et al. 1996, Garcés Vernier et al. 2000], y en el rango de los nanómetros para partículas metálicas [Scaffardi et al. 2005, Scaffardi and Tocho 2006, Scaffardi et al. 2007, Scaffardi and Tocho 2008, Schinca and Scaffardi 2008, Roldán et al. 
2008, Torchia et al. 2008, Schinca et al. 2009, Videla et al. 2009, Videla et al. 2010, Abraham Ekeroth et al. 2011] en suspensiones líquidas diluidas.

La Figura 5.4 representa una posible configuración genérica de la situación. Cuando el detector se coloca en la dirección de la luz incidente, después de atravesar el conjunto de partículas, se puede medir un flujo de energía por unidad de área que es menor que la que presenta la onda incidente. En el caso más general la extinción, que es la suma de scattering + absorción, presenta contribución de los dos términos, pero como las partículas tienen un tamaño mucho menor que la longitud de onda incidente, el fenómeno de absorción predomina sobre el de scattering, como se ha mencionado en la Sección 2.1.2.

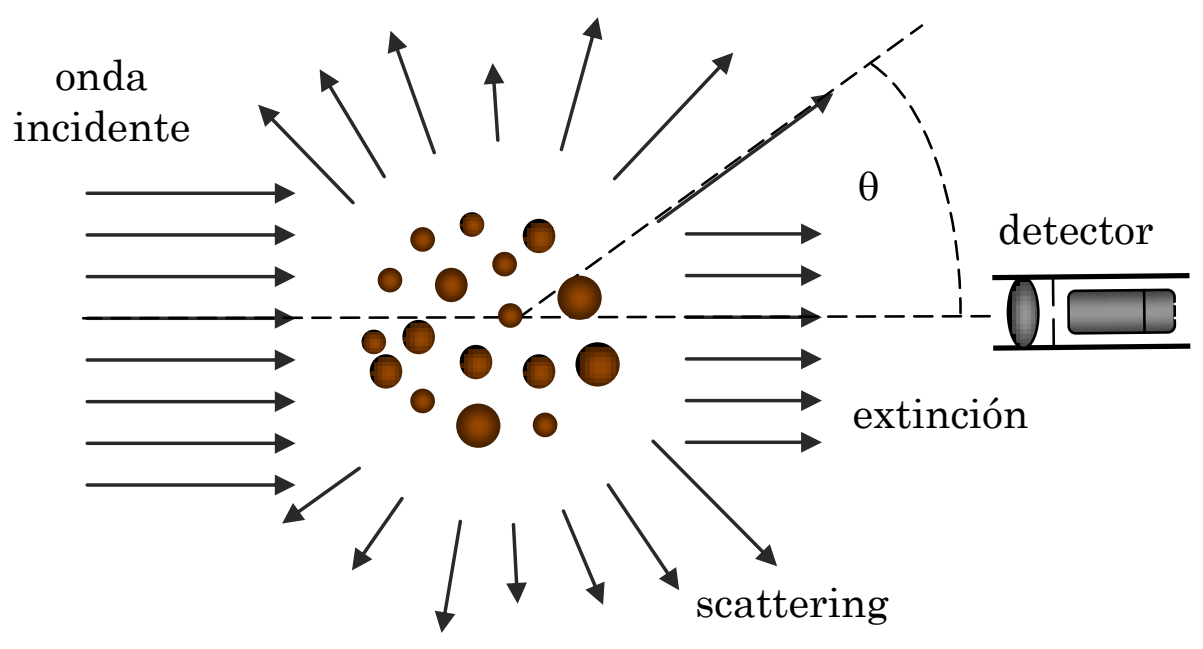

Figura 5.4: Esquema de los procesos de scattering y extinción de un conjunto de nanopartículas metálicas.

Los resultados experimentales serán ajustados por medio de los cálculos de Mie para partículas esféricas, donde es necesario conocer el índice de refracción en función de la longitud de onda, tanto para el material particulado como para el solvente. Para la función dieléctrica del metal 
utilizada en este cálculo de Mie, es necesario incluir las modificaciones de tamaño de la contribución de los electrones libres y ligados descriptas en la Sección 3.3.

Un espectrofotómetro mide la extinción de luz, que es la fracción de la luz removida del haz incidente. Operativamente, la extinción puede ser derivada de la relación dada por la ley de Lambert-Beer:

$$
I_{t}=I_{i} \exp \left(-\beta_{\text {ext }} l\right)
$$

donde $I_{i}$ es la intensidad incidente sobre la muestra, $I_{t}$ es la transmitida, $\beta_{\text {ext }}$ es el coeficiente de atenuación y $l$, la longitud de la muestra. Cuando la dilución de la muestra es tal que la distancia promedio entre partículas en el medio es lo suficientemente grande como para que pueda considerarse el scattering incoherente, es posible relacionar el coeficiente de atenuación de una muestra de $N$ partículas por unidad de volumen, con la sección eficaz de partícula aislada $C_{e x t}$ :

$$
\beta_{\text {ext }}=N C_{\text {ext }}
$$

Los llamados "espectros de extinción" se obtienen realizando un conjunto de mediciones de $I_{t} / I_{i}$ en un intervalo de longitudes de onda determinado. Los resultados pueden compararse con la eficiencia espectral de extinción calculada, $Q_{\text {ext }}(\lambda)$ para un dado índice de refracción del medio y radio de nanopartícula, quien es utilizado como parámetro de ajuste. Esta firma espectral contiene información de la composición química de la muestra así como la distribución de tamaños de las partículas.

Los resultados experimentales pueden ser fuertemente alterados a menos que se preste especial atención para evitar que el scattering 
indeseado ingrese al detector. Un agujero de pequeño diámetro colocado en una posición adecuada entre la muestra y el detector ayudará a mejorar la fiabilidad de las mediciones.

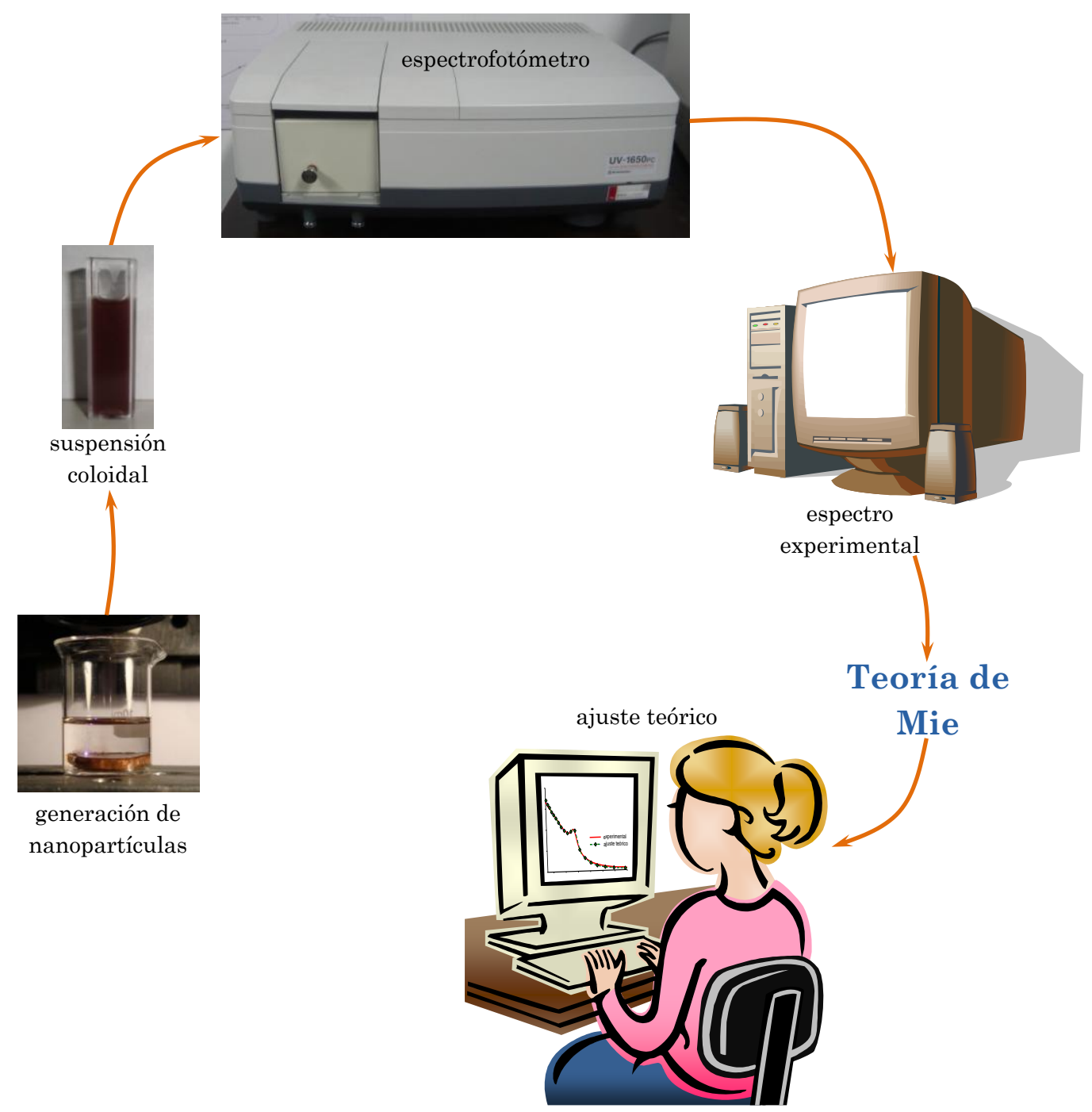

Figura 5.5: Esquema del procedimiento experimental completo de la generación y análisis de los espectros experimentales de las suspensiones coloidales de cobre y plata.

Es interesante mencionar que las partículas metálicas de tamaños muy pequeños presentan un scattering despreciable frente al de absorbancia, de manera que la extinción es prácticamente igual a la 
absorbancia. Por esta razón, la medida de absorbancia obtenida con un espectrofotómetro podrá ser ajustada por teoría de Mie para obtener información sobre la distribución de tamaños de las suspensiones coloidales de metales nobles.

La Figura 5.5 muestra un esquema del procedimiento experimental completo, realizado para la obtención del espectro experimental de las suspensiones coloidales de cobre y plata generadas por ablación láser y su posterior ajuste teórico mediante teoría de Mie.

\subsection{Técnicas complementarias de análisis}

A modo de complementar la técnica de espectroscopía de extinción óptica se utilizó, para la determinación de la composición de nanopartículas tipo core-shell, la técnica de espectroscopía Raman. Como es sabido, el scattering Raman se caracteriza por poseer componentes de frecuencia diferentes a la frecuencia incidente no resonante, cuyas diferencias (“corrimientos Raman") guardan relación directa con transiciones resonantes moleculares de determinado compuesto (en el caso de soluciones) o con excitaciones fonónicas (en sólidos o suspensiones de sólidos en líquidos). Estas transiciones resonantes yacen por lo general en el IR lejano, lo que dificulta su detección por la baja eficiencia de los detectores en esa región del espectro. Como la radiación incidente más comúnmente usada en este tipo de experiencias es un láser en la región del visible, la espectroscopía Raman permite medir las transiciones del IR lejano a esta región del espectro (a través de la medición de los corrimientos Raman), donde los detectores poseen alta eficiencia. En este trabajo de Tesis se utilizó un láser de Nd:YAG doblado en frecuencia $(\lambda=532 \mathrm{~nm})$, que se enfocaba sobre una celda de cuarzo conteniendo la suspensión de 
nanopartículas. La observación se realizó a $90^{\circ}$, a través de una fibra óptica que se conectaba a la entrada de un espectrógrafo con cámara CCD refrigerada con intensificador. La Figura 5.6 muestra una fotografía del dispositivo experimental.

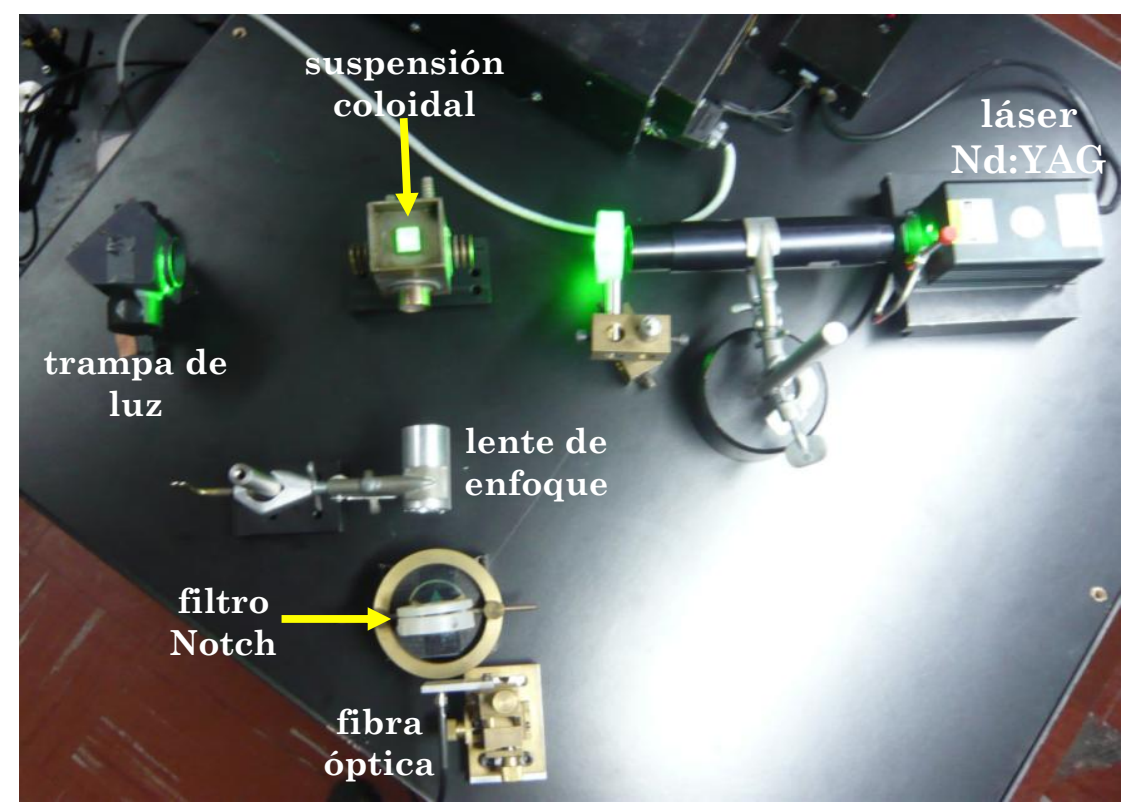

Figura 5.6: Dispositivo experimental para la obtención de espectros Raman de suspensiones coloidales de nanopartículas metálicas.

Para obtener los "corrimientos Raman" $(\Delta v)$ en unidades de $\mathrm{cm}^{-1}$ se utilizó la siguiente expresión:

$$
\Delta v=10^{7}\left[\frac{1}{\lambda_{0}}-\frac{1}{\lambda_{R}}\right]
$$

donde $\lambda_{0}$ es la longitud de onda del láser y $\lambda_{R}$ corresponde a las longitudes de onda de las transiciones resonantes. 
Finalmente, para tener una medición de comparación de tamaño de partícula, las muestras se analizaron usando Microscopía de Fuerza Atómica (AFM). Las suspensiones coloidales obtenidas por ablación láser se diluyeron en una relación 1:100. Una gota de la solución diluida fue puesta sobre la superficie de una mica de alta calidad Ruby Mica Disc (rugosidad media de $0,073 \mathrm{~nm}$, según lo declarado por el fabricante). Las observaciones fueron realizadas sobre la muestra seca con un microscopio AFM NT-MDT Solver Pro (paso mínimo de scanning vertical de 0,006 nm) que trabaja en el modo de semicontacto, utilizando un cantilever triangular NSG20 (frecuencia de resonancia $534 \mathrm{~Hz}$ ) con punta de curvatura de $10 \mathrm{~nm}$ de radio. También se realizaron mediciones de contrastación de tamaño con microscopía electrónica de alta resolución, HRTEM (JEOL 4000EX). 


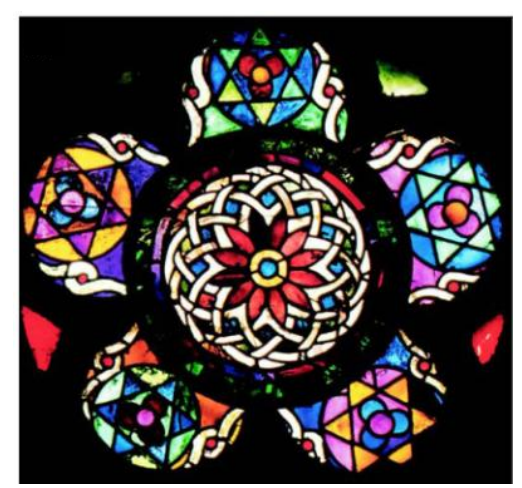

Monasterio de Santes Creus Tarragona, España

\section{Capítulo 6}

\section{Caracterización óptica de nanoestructuras metálicas esféricas}

En los últimos años, ha habido un creciente interés en el estudio de nanoestructuras de metales de transición, debido a su capacidad de reforzar los campos ópticos en la escala nanométrica, lo que las hace interesantes para diversas aplicaciones en varios campos de la ciencia y la tecnología. Algunos ejemplos que se pueden mencionar de la aplicación de estas nanoestructuras son los sensores químicos y biológicos [Cao et al. 2002, Nath and Chilkoti 2002, Riboh et al. 2003, Anker et al. 2008, Stewart 2008, Kneipp et al. 2010], como así también los dispositivos optoelectrónicos y plasmónicos [Alivisatos et al. 1998, Shipway et al. 2000, Xia et al. 2000, Li et al. 2003, Maier et al. 2003, Zou and Schatz 2006], entre otras.

En estas áreas de investigación y desarrollo, es indispensable la caracterización óptica de las nanoestructuras a través del estudio de la función dieléctrica y de los espectros de extinción, y a su vez utilizar esta información para controlar sus propiedades plasmónicas. 
En este Capítulo se analiza teóricamente en forma separada la contribución de los electrones libres y ligados a la función dieléctrica compleja corregida por tamaño para nanopartículas de cobre y plata. Se determinan los parámetros relacionados a la contribución de los electrones ligados para ambos metales de tamaño macroscópico y se realiza un análisis del comportamiento de los espectros de extinción en función de la longitud de onda para distintos tamaños de partículas nanométricas y subnanométricas y diferentes estructuras simples y núcleo-recubrimiento.

Se muestran los ajustes de los espectros de extinción experimentales de las suspensiones coloidales de cobre y plata generadas por ablación láser de pulsos ultracortos, para determinar la composición, estructura, configuración y distribución de tamaños.

\section{Nanopartículas esféricas de cobre y plata}

El estudio del comportamiento de nanopartículas de cobre y plata es un área de investigación activa debido a sus potenciales aplicaciones en química, catálisis, ciencia de los materiales y nanofluidos [Larsen and Noriega 2004, Wang et al. 2004, Patel et al. 2005, Zhu et al. 2005]. La alta conductividad, sección eficaz de extinción, fotosensibilidad y bajo costo del cobre, lo hacen un material prometedor para el desarrollo de dispositivos miniaturizados que puedan integrar características electrónicas, fotónicas y químicas para su utilización en nanosensores biológicos [Jiang et al. 2003, Haes et al. 2004, Moran et al. 2005, Dieringer et al. 2006, Zhao et al. 2006]. También es interesante su capacidad de inserción en matrices poliméricas huésped para aplicaciones ópticas no lineales [Gotoh et al. 2000, Quaranta et al. 2004, Rostovshchikova et al. 2005]. 
En particular las nanopartículas de plata de estructura core-shell resultan interesantes por su capacidad para mejorar las propiedades de conductividad térmica y eléctrica en adhesivos conductores anisotrópicos al ser incorporadas en forma de monocapas [ $[\mathrm{Li}$ et al. 2005] o de nanorecubrimientos de óxido de plata y núcleo de plata en nanocompósitos ("nanocomposites") de silicona [Chatterjee et al. 2002]. En el área de la biología, las nanopartículas de plata cumplen un rol muy importante debido a sus propiedades antivirales y bactericidas, siendo un campo de investigación activo. Investigaciones de los últimos años [Elechiguerra et al. 2005] han mostrado que nanopartículas de plata con recubrimiento, en el rango de 1 a $10 \mathrm{~nm}$, experimentan una interacción dependiente del tamaño con el HIV-1, atacando al virus e inhibiéndolo de combatir a las células huésped.

\subsection{Determinación de parámetros de electrones ligados de $\mathrm{Cu}$ y $\mathrm{Ag}$ en la función dieléctrica}

Para metales nobles, las transiciones de los electrones ligados hacia los niveles de la banda de conducción contribuyen apreciablemente a la función dieléctrica como se ha discutido en la Sección 3.3.4. Pinchuck y coautores [Pinchuck et al. 2004] han analizado la influencia de las transiciones electrónicas interbanda sobre diferentes parámetros relacionados con la función dieléctrica y la resonancia del plasmón (frecuencia, amplitud y ancho medio) en pequeños clusters de metales nobles utilizando la aproximación de Rayleigh. Sin embargo, la influencia de los electrones libres y ligados dependientes del tamaño en la función dieléctrica y en el coeficiente de extinción, no ha sido completamente estudiada para los 
casos de nanopartículas de cobre y plata. Esto se analiza en detalle en la siguiente sección.

\subsubsection{Función dieléctrica de $\mathrm{Cu}$ y Ag dependiente del tamaño}

A partir del modelado teórico de la función dieléctrica compleja de cobre y plata, considerando la corrección por tamaño en las contribuciones de los electrones libres y ligados como se ha desarrollado en el Capítulo 3, fue posible ajustar la función dieléctrica bulk experimental medida por Johnson y Christy [Johnson and Christy 1972] para ambos metales.

El ajuste simultáneo de las partes real e imaginaria de la función dieléctrica macroscópica experimental a partir del cálculo teórico basado en la suma de ambas contribuciones, utilizando las ecuaciones (3.15), (3.21), y (3.37), teniendo en cuenta $a=100 \mathrm{~nm}$ (como fue mencionado en la Sección 3.3.4), ha permitido determinar los valores óptimos para $K_{b u l k}, E_{g}, E_{F} \mathrm{y}$ $\gamma_{\text {ligados }}$ del cobre y la plata.

\section{- Cobre}

La Figura 6.1 muestra por un lado las componentes real (a) e imaginaria (b), de las contribuciones de los electrones libres (ecuación (3.21)) y ligados (ecuación (3.37)) calculadas separadamente. Por otro lado se muestra la suma teórica de ambas contribuciones (línea sólida), que corresponde al mejor ajuste de los valores experimentales de cobre macroscópico (círculos) tomados de Johnson y Christy.

Se observa una excelente concordancia entre los valores calculados teóricamente y los datos experimentales. 

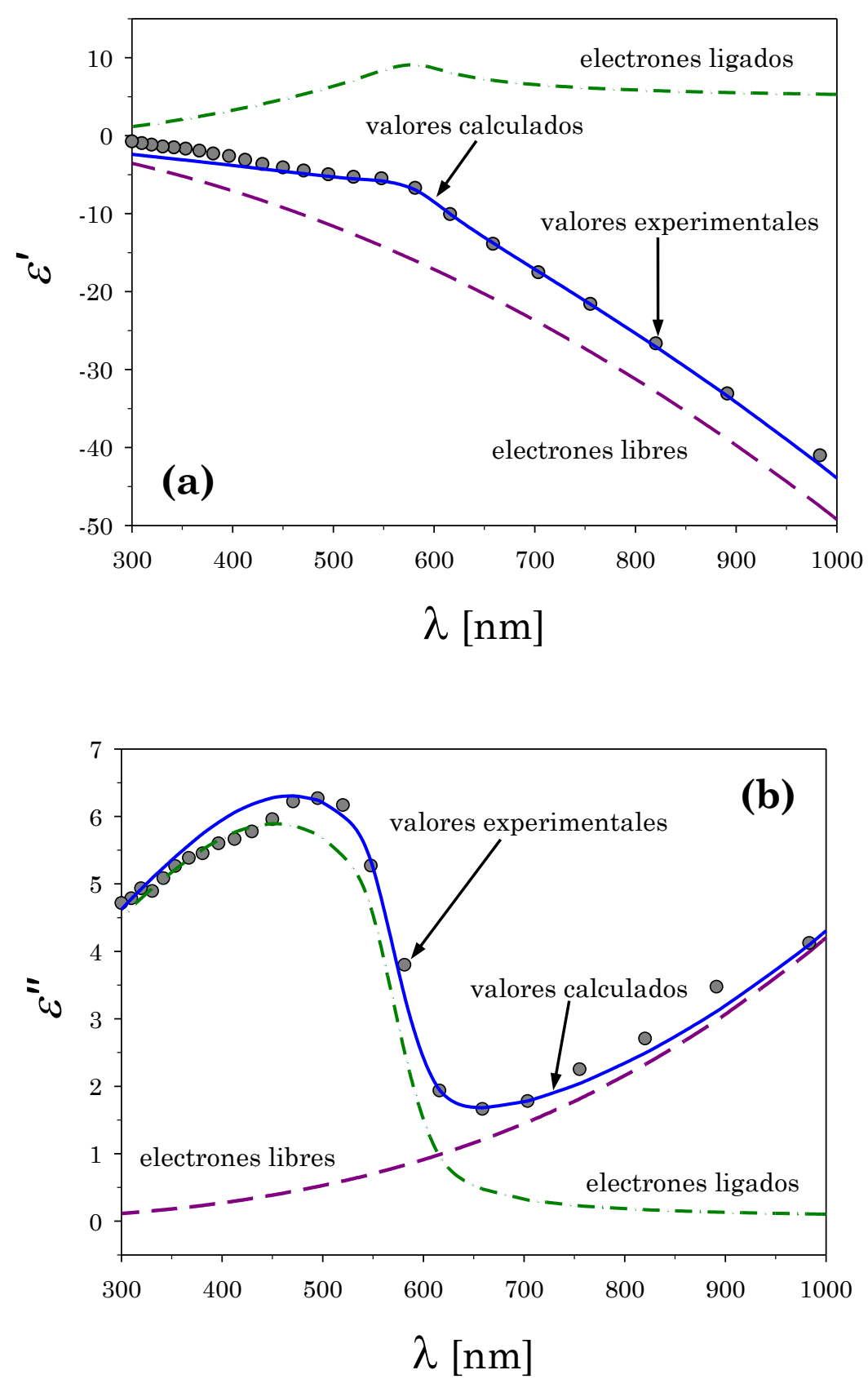

Figura 6.1: Comparación entre los valores experimentales (círculos) y los cálculos teóricos de las partes real (a) e imaginaria (b) de la función dieléctrica bulk del cobre (ecuación (3.15)). Los valores experimentales fueron tomados de Johnson y Christy, mientras que los valores teóricos de las contribuciones de los electrones libres y ligados a la función dieléctrica compleja fueron calculados usando las ecuaciones (3.21) y (3.37), y los parámetros dados en la Tabla 6.1. 
Es interesante observar en la Figura 6.1 (b) que la contribución de los electrones ligados domina sobre la contribución de los electrones libres para longitudes de onda por debajo de $600 \mathrm{~nm}$, mientras que para longitudes de onda por encima de $600 \mathrm{~nm}$ la contribución que empieza a influir es la de los electrones libres. Este comportamiento particular da lugar al aspecto característico de la curva que corresponde a los valores experimentales e influye en los espectros de extinción ópticos de las partículas nanométricas y subnanométricas de cobre en el rango de longitudes de onda cortas.

Los parámetros bibliográficos utilizados para el ajuste y los calculados en este trabajo de Tesis se resumen en la Tabla 6.1.

Tabla 6.1: Parámetros del cobre utilizados y determinados en este trabajo

\begin{tabular}{|c|c|c|c|}
\hline Parámetros del Cu & Símbolos & Valores & Referencias \\
\hline Frecuencia de plasma & $\omega_{p}$ & $13,4 \times 10^{15} \mathrm{~Hz}$ & $\begin{array}{c}\text { [Cai and Shalaev } \\
\text { 2010] }\end{array}$ \\
\hline $\begin{array}{l}\text { Constante de amortiguamiento } \\
\text { para los electrones libres }\end{array}$ & $\gamma_{\text {libres }}$ & $1,45 \times 10^{14} \mathrm{~Hz}$ & $\begin{array}{l}\text { [Johnson and } \\
\text { Christy 1972] }\end{array}$ \\
\hline Velocidad de Fermi & $v_{F}$ & $15,7 \times 10^{14} \mathrm{~nm} / \mathrm{s}$ & $\begin{array}{l}\text { [Kaye and Laby } \\
\text { 1995] }\end{array}$ \\
\hline $\begin{array}{l}\text { Coeficiente de la contribución } \\
\text { del electrón ligado }\end{array}$ & $K_{\text {bulk }}$ & $2 \times 10^{24}$ & $\begin{array}{l}\text { [Santillán et al. } \\
\text { 2012a] }\end{array}$ \\
\hline Energía del gap & $E_{g}$ & $1,95 \mathrm{eV}$ & $\begin{array}{l}\text { [Santillán et al. } \\
\text { 2012a] }\end{array}$ \\
\hline Energía de Fermi & $E_{F}$ & $2,15 \mathrm{eV}$ & $\begin{array}{l}\text { [Santillán et al. } \\
\qquad 2012 \mathrm{a}]\end{array}$ \\
\hline $\begin{array}{l}\text { Constante de amortiguamiento } \\
\text { para los electrones ligados }\end{array}$ & $\gamma_{\text {ligados }}$ & $1,15 \times 10^{14} \mathrm{~Hz}$ & $\begin{array}{l}\text { [Santillán et al. } \\
\text { 2012a] }\end{array}$ \\
\hline
\end{tabular}


Usando el modelo de la función dieléctrica descripto en la Sección 3.3, se han determinado por primera vez los parámetros microscópicos $K_{b u l k} \mathrm{y}$ $\gamma_{\text {ligados }}$ vinculados a la contribución de los electrones ligados [Santillán et al. 2012a, Santillán et al. 2012b]. Con este procedimiento de ajuste espectral, se obtuvieron también los valores de $E_{F}$ y $E_{g}$ que coinciden, dentro de los errores experimentales, con los reportados por otros autores y con otros métodos [Fox 2001]. Esto permite otorgar confiabilidad al método de ajuste de la función dieléctrica macroscópica, como así también a la determinación de los valores de $K_{\text {bulk }} \mathrm{y} \gamma_{\text {ligados }}$ obtenidos por este procedimiento.

Teniendo en cuenta los parámetros $K_{\text {bulk }}, \quad E_{g}, \quad E_{F}$ y $\gamma_{\text {ligados }}$ determinados más arriba, es importante ahora analizar el comportamiento teórico de la contribución de los electrones libres y ligados en función del radio para valores nanométricos y subnanométricos incluyendo las correcciones correspondientes $\gamma_{\text {libres }}^{t}$ y $K_{t}$ mencionadas en la Sección 3.3, para luego estudiar su posterior influencia en los espectros de extinción.

La Figura 6.2 representa el comportamiento de las partes real (a) e imaginaria (b) de la contribución de los electrones libres a la función dieléctrica de cobre, para diferentes radios de partículas, utilizando las expresiones (3.29) y (3.30).

Se puede observar en la Figura 6.2 (a) que la componente real de la contribución de los electrones libres a la función dieléctrica es muy sensible para $a<2 \mathrm{~nm}$. En el rango de 2 a $10 \mathrm{~nm}$ es mucho menos sensible y casi coincidente con la curva correspondiente a bulk para $a=10 \mathrm{~nm}$. Por otra parte, la componente imaginaria representada en la Figura 6.2 (b) muestra un comportamiento límite similar al bulk para $a>20 \mathrm{~nm}$, exhibiendo gran sensibilidad para valores de radios en el rango $0,7 \mathrm{~nm}<a<10 \mathrm{~nm}$ y 
presenta un comportamiento límite inferior para valores que están por debajo de 0,6 $\mathrm{nm}$.
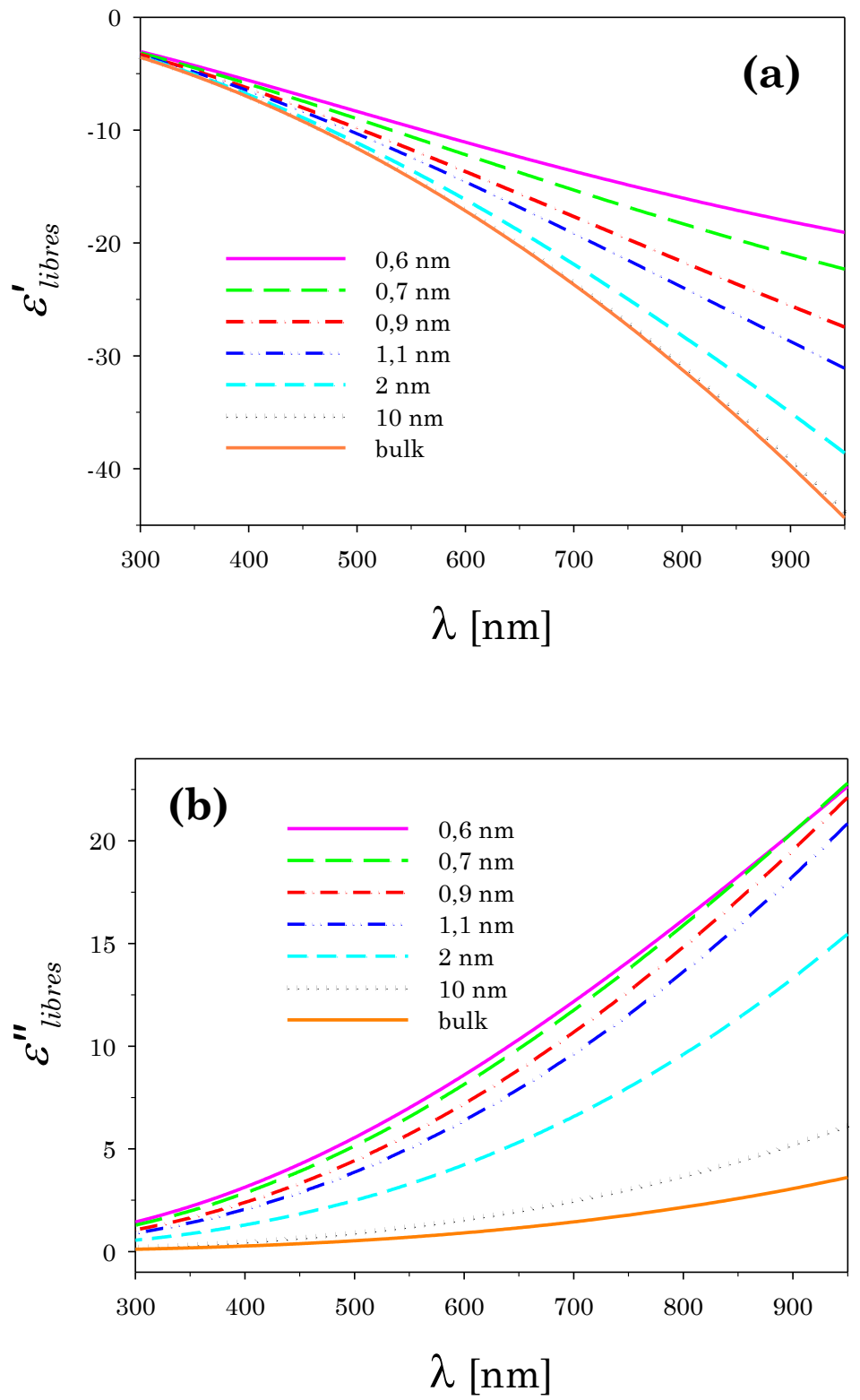

Figura 6.2: Contribución de los electrones libres a la función dieléctrica del cobre en función de la longitud de onda para diferentes radios. (a) Componente real y (b) componente imaginaria, calculadas con las ecuaciones (3.29) y (3.30), respectivamente. 
Las Figuras 6.3 (a) y (b) muestran las partes real e imaginaria de la contribución de los electrones ligados a la función dieléctrica dependiente del tamaño, para diferentes valores de radio utilizando la ecuación (3.37) con la corrección $K_{t}=K_{b u l k}\left(1-\exp \left(-a / a_{0}\right)\right)$ mencionada en la Sección 3.3.4.1.
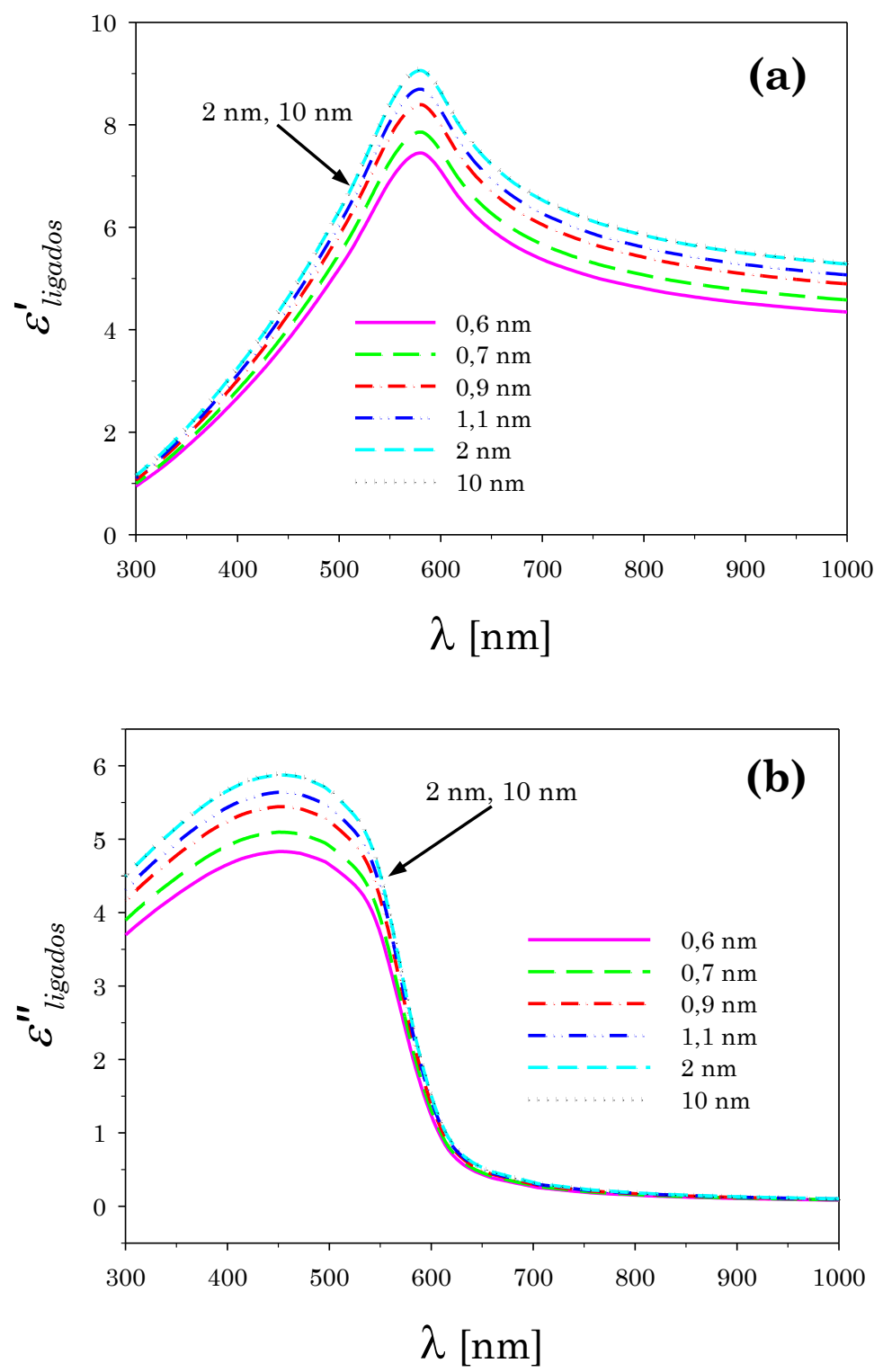

Figura 6.3: Contribución de los electrones ligados a la función dieléctrica del cobre en función de la longitud de onda para diferentes radios. (a) Componente real y (b) componente imaginaria, calculadas con la ecuación (3.37) teniendo en cuenta la corrección por tamaño. 
En la Figura 6.3 (a) se observa que las curvas son muy sensibles para diferentes radios por debajo de $1 \mathrm{~nm}$, pero la corrección por tamaño de los electrones ligados se hace despreciable para $a \geq 2 \mathrm{~nm}$, observándose que las curvas de $2 \mathrm{~nm}$ y $10 \mathrm{~nm}$ aparecen superpuestas a lo largo de todo el rango UV-visible analizado. En la Figura 6.3 (b), la parte imaginaria muestra un comportamiento límite similar con el tamaño, siendo también despreciable la corrección para valores del radio $a \geq 2 \mathrm{~nm}$.

Hasta el momento se han mostrado los comportamientos de las contribuciones de los electrones libres (Figura 6.2) y ligados (Figura 6.3) a la función dieléctrica dependientes del tamaño por separado. En la Figura 6.4 se representan la componente real (a) e imaginaria (b) de la función dieléctrica total (ecuación (3.15)) como función de la longitud de onda para diferentes tamaños de partículas nanométricas y subnanométricas de $\mathrm{Cu}$.

Se observa en la Figura 6.4 (a) que la componente real de la función dieléctrica total de $\mathrm{Cu}$ presenta gran sensibilidad para radios entre 0,6 y $10 \mathrm{~nm}$ por encima de $550 \mathrm{~nm}$ de longitud de onda. Para valores de radio mayores que $10 \mathrm{~nm}$, la función dieléctrica coincide con la correspondiente al bulk.

En la Figura 6.4 (b) se puede ver que la parte imaginaria de la función dieléctrica total corregida por tamaño presenta variaciones significativas para radios comprendidos entre 0,7 y $10 \mathrm{~nm}$ aproximadamente para todo el rango UV-visible analizado.

El comportamiento de la función dieléctrica coincide con el que exhibe el bulk ya para $a=20 \mathrm{~nm}$. Como fue mencionado anteriormente, este modelo de la función dieléctrica presenta para el caso del $\mathrm{Cu}$ un límite inferior para 0,6 $\mathrm{nm}$ de radio, tamaño para el cual la curva comienza a solaparse con la que corresponde a $0,7 \mathrm{~nm}$ para longitudes de onda por encima de $800 \mathrm{~nm}$. 

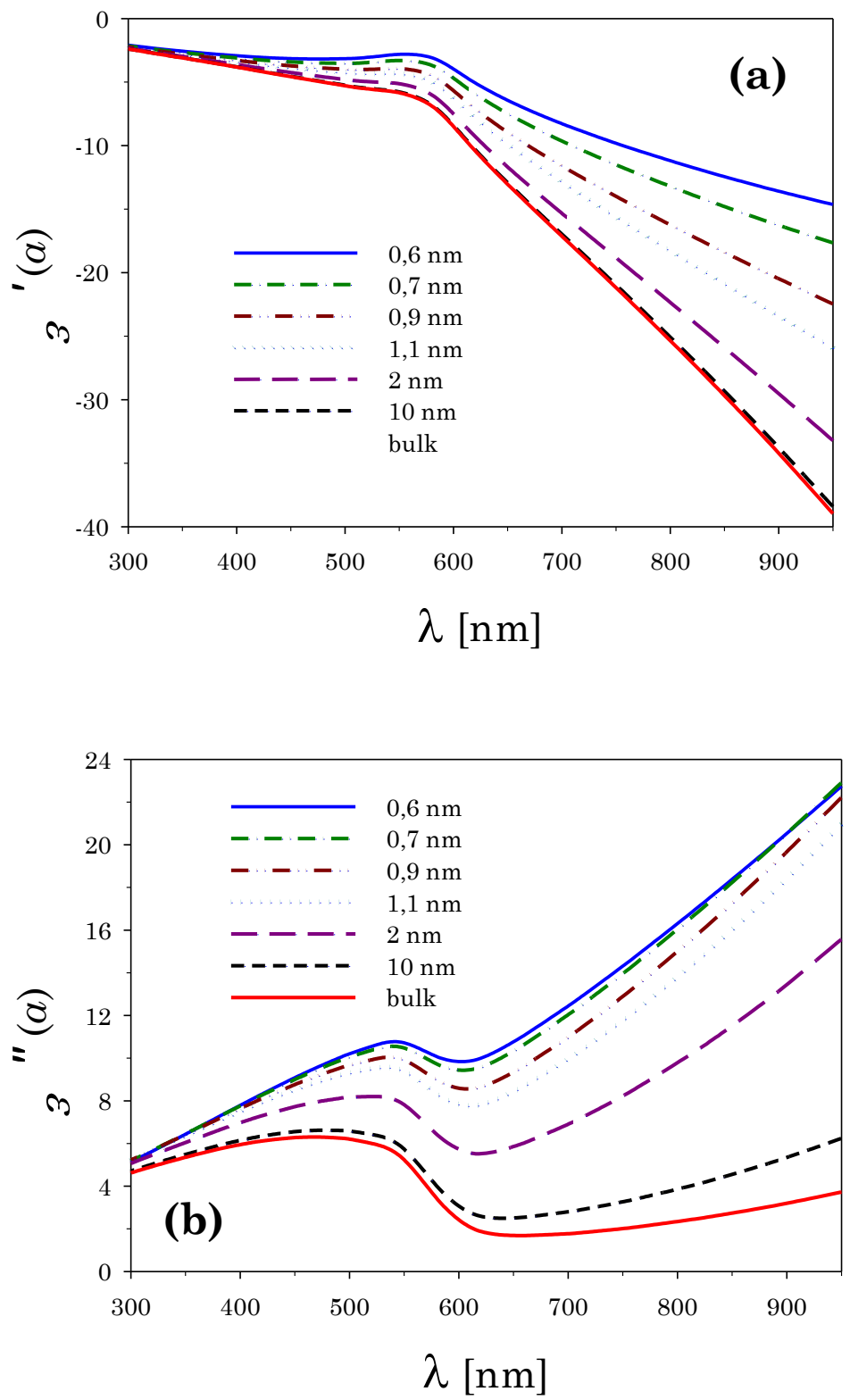

Figura 6.4: Componentes real (a) e imaginaria (b) de la función dieléctrica completa del cobre. La curva roja representa los datos experimentales tomados de Johnson y Christy. Las curvas restantes representan la corrección por tamaño para partículas de 0,6 a $10 \mathrm{~nm}$ de radio.

En esta etapa, una vez conocida la forma de la función dieléctrica compleja bulk del cobre, es importante analizar la influencia que presentan los parámetros $E_{g}, E_{F}, \gamma_{\text {ligados }}$ y $K_{\text {bulk }}$ en el comportamiento de la misma. 
En las Figuras 6.5 a 6.8 se analizan las variaciones producidas en las partes real e imaginaria debido a pequeños cambios de los mencionados parámetros, en el orden indicado en la frase anterior.
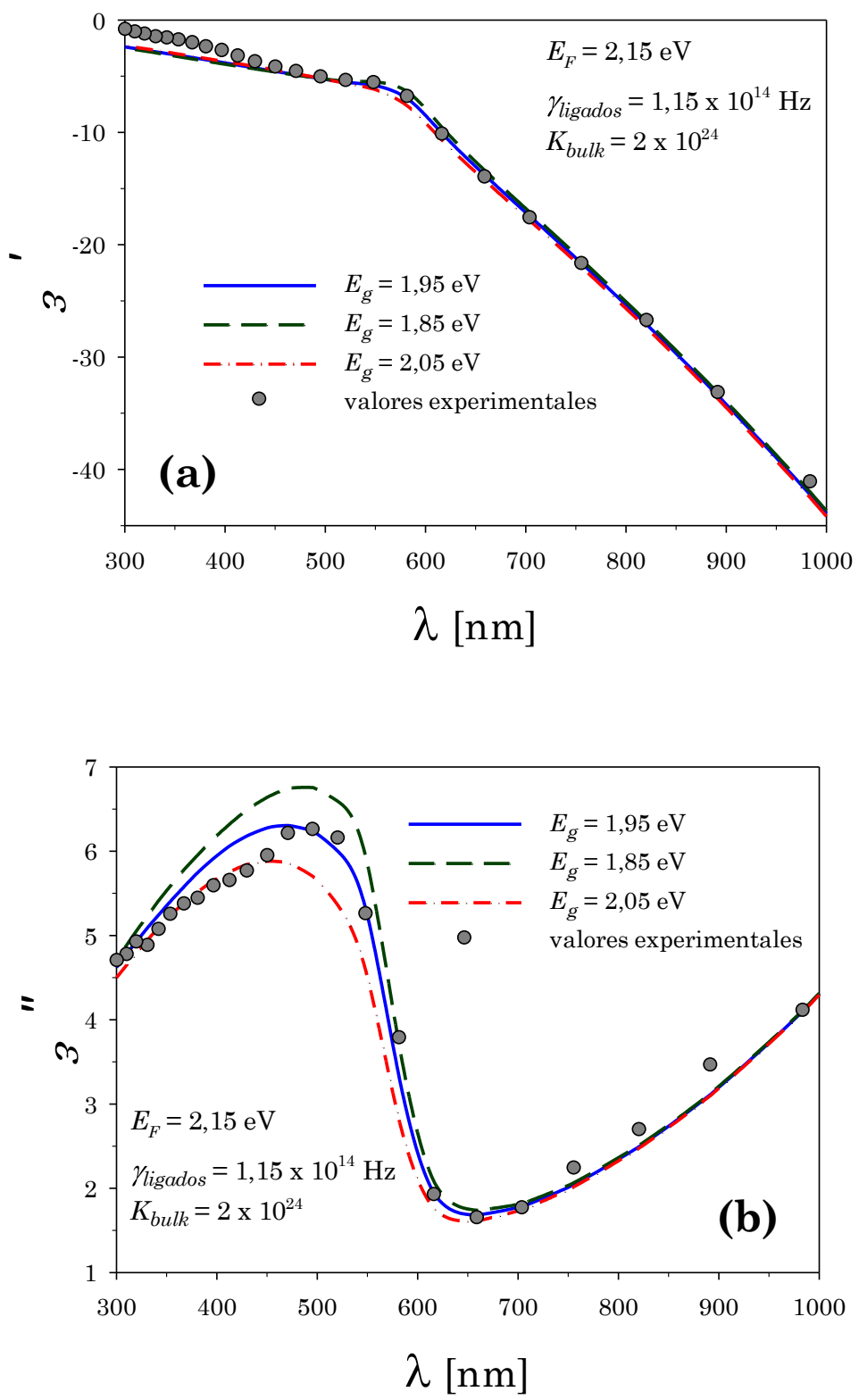

Figura 6.5: Influencia de $E_{g}$ en la función dieléctrica compleja bulk del cobre: (a) componente real y (b) componente imaginaria. La influencia en la parte imaginaria es más notable para longitudes de onda en el rango de 350 a 530 nm (región I). 
La Figura 6.5 muestra la influencia de $E_{g}$ en la función dieléctrica compleja. Aunque la parte real parece no ser tan sensible a los cambios en $E_{g}$, existe una diferencia notable en las curvas calculadas para la parte imaginaria (Figura 6.5 (b)), especialmente en la región de longitudes de onda entre 350 y $530 \mathrm{~nm}$ (región I), correspondiente al comienzo de la transición del gap.

En la Figura 6.6 se representa la influencia de $E_{F}$ en la función dieléctrica compleja bulk del cobre. Se puede notar que para pequeñas variaciones en el valor de $E_{F}$, la parte real (Figura 6.6 (a)) muestra un cambio casi imperceptible entre longitudes de onda de 530 a 700 nm (región II). Sin embargo, los cambios más significativos ocurren en la parte imaginaria (Figura 6.6 (b)) entre 530 y $700 \mathrm{~nm}$, la cual corresponde al borde de la transición. Por encima de $700 \mathrm{~nm}$ (región III) la influencia de los electrones ligados es casi nula, siendo esta región más influenciada por la contribución de los electrones libres.

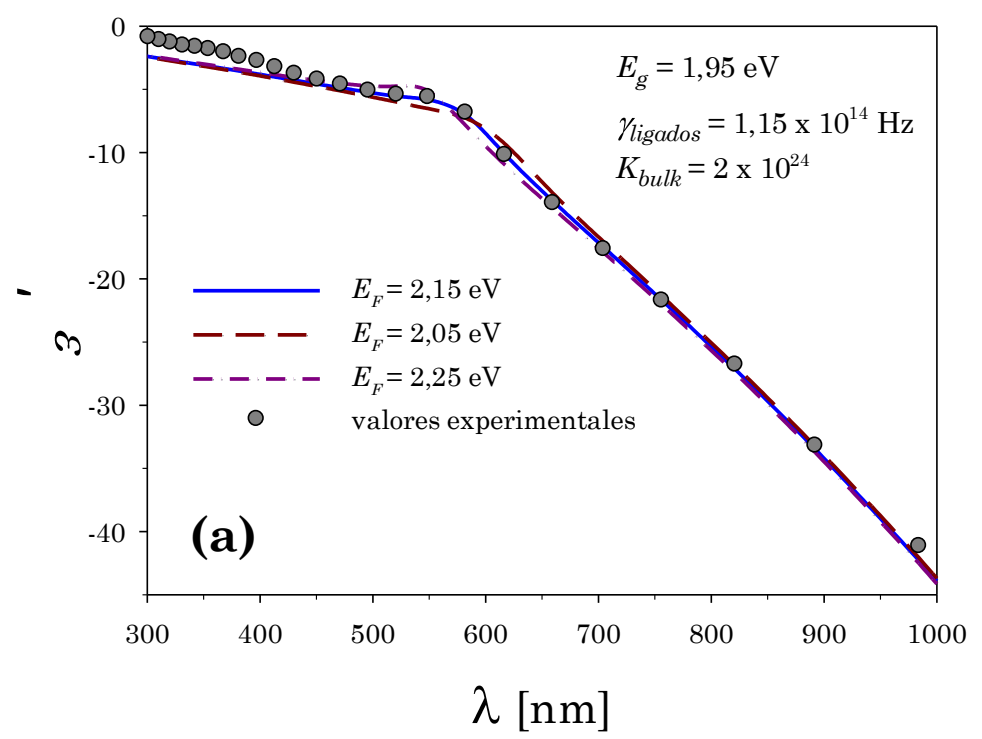




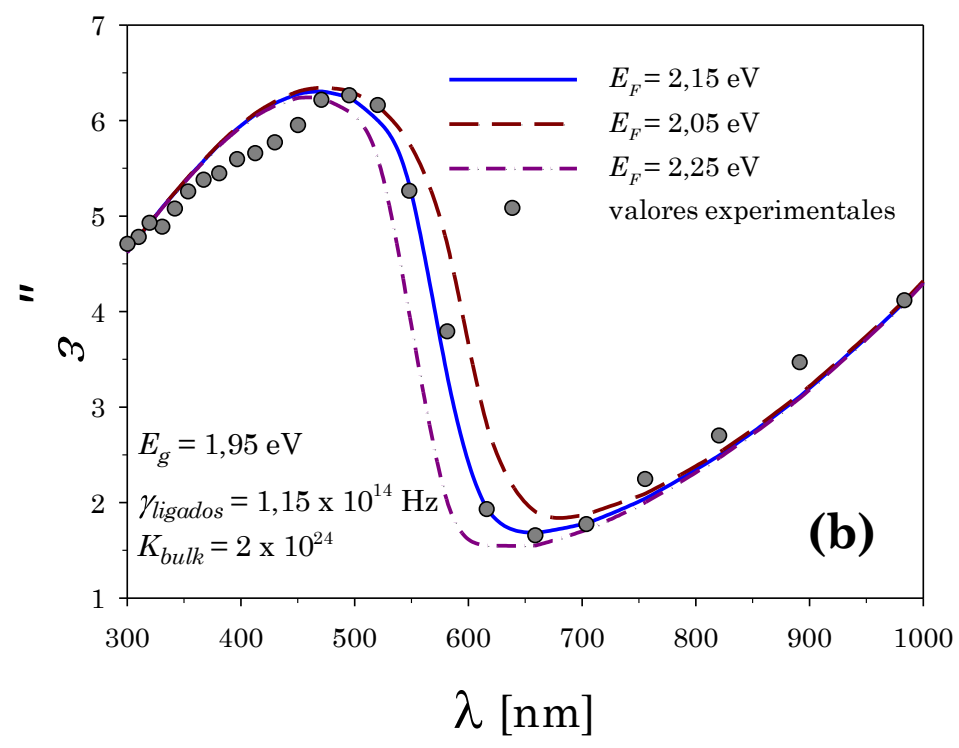

Figura 6.6: Influencia de $E_{F}$ en la función dieléctrica compleja bulk del cobre: (a) parte real y (b) imaginaria. La influencia en la parte imaginaria es más significativa entre 530 y $750 \mathrm{~nm}$ (región II).

La Figura 6.7 muestra la influencia de las pequeñas modificaciones de $\gamma_{\text {ligados }}$ sobre las partes real e imaginaria de la función dieléctrica bulk del cobre.

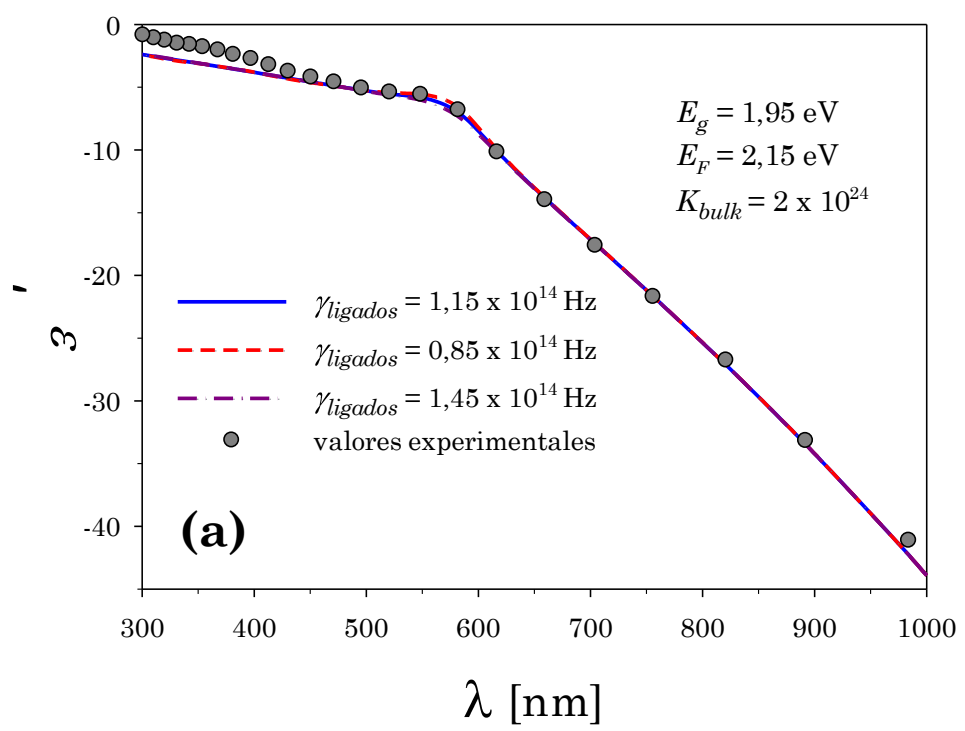




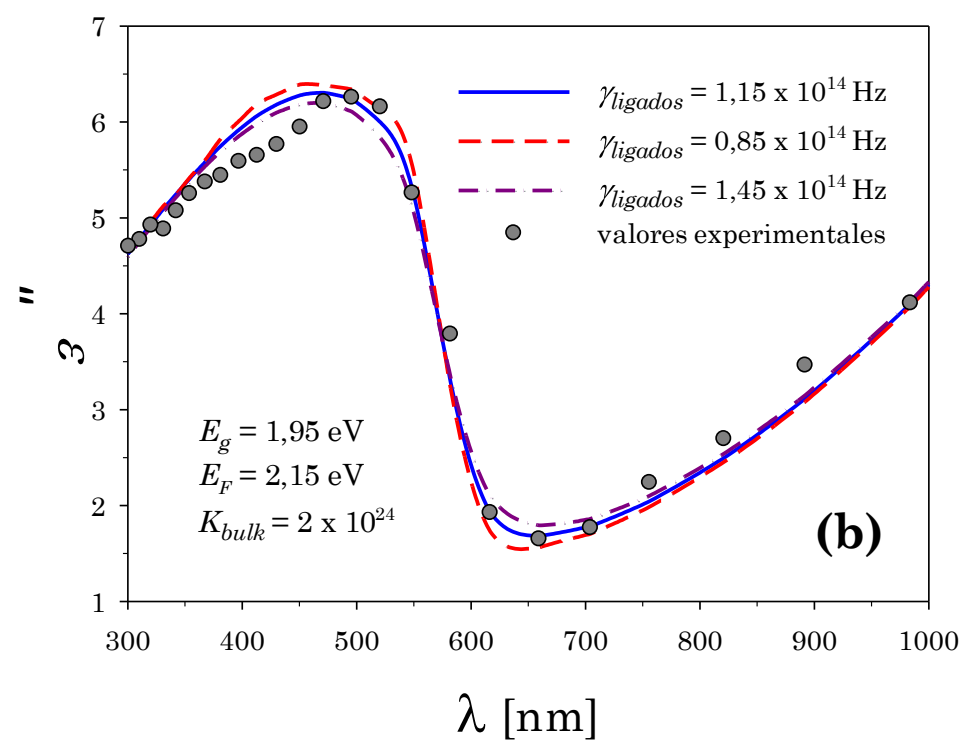

Figura 6.7: Influencia de $\gamma_{\text {ligados }}$ en la función dieléctrica compleja bulk del cobre: (a) parte real y (b) imaginaria. Las zonas que muestran cambios más significativos debido a la influencia de $\gamma_{\text {ligados }}$ afectan principalmente al pico y valle de la curva en la parte imaginaria.

Aquí nuevamente, mientras que la parte real (a) es casi imperceptible a los cambios en $\gamma_{\text {ligados }}$, la parte imaginaria (b) muestra comportamientos notables en las zonas del pico y del valle.

Finalmente, en la Figura 6.8 se representa la influencia de $K_{b u l k}$ en la función dieléctrica compleja bulk del cobre. Se puede observar que para pequeños cambios en el valor de $K_{b u l k}$, la parte imaginaria (Figura 6.8 (b)) presenta variaciones distinguibles por debajo de 530 nm (región I).

Luego del análisis pormenorizado de la influencia que ejercen los parámetros $E_{g}, E_{F}, \gamma_{\text {ligados }}$ y $K_{b u l k}$ sobre la función dieléctrica compleja bulk del cobre, se observa que los cambios en las parte real o imaginaria producidos por cada uno de ellos influye en regiones espectrales diferentes e independientes. Esta característica permite asegurar que la optimización de 
los parámetros antes mencionados para el ajuste de la función dieléctrica sea unívoca.
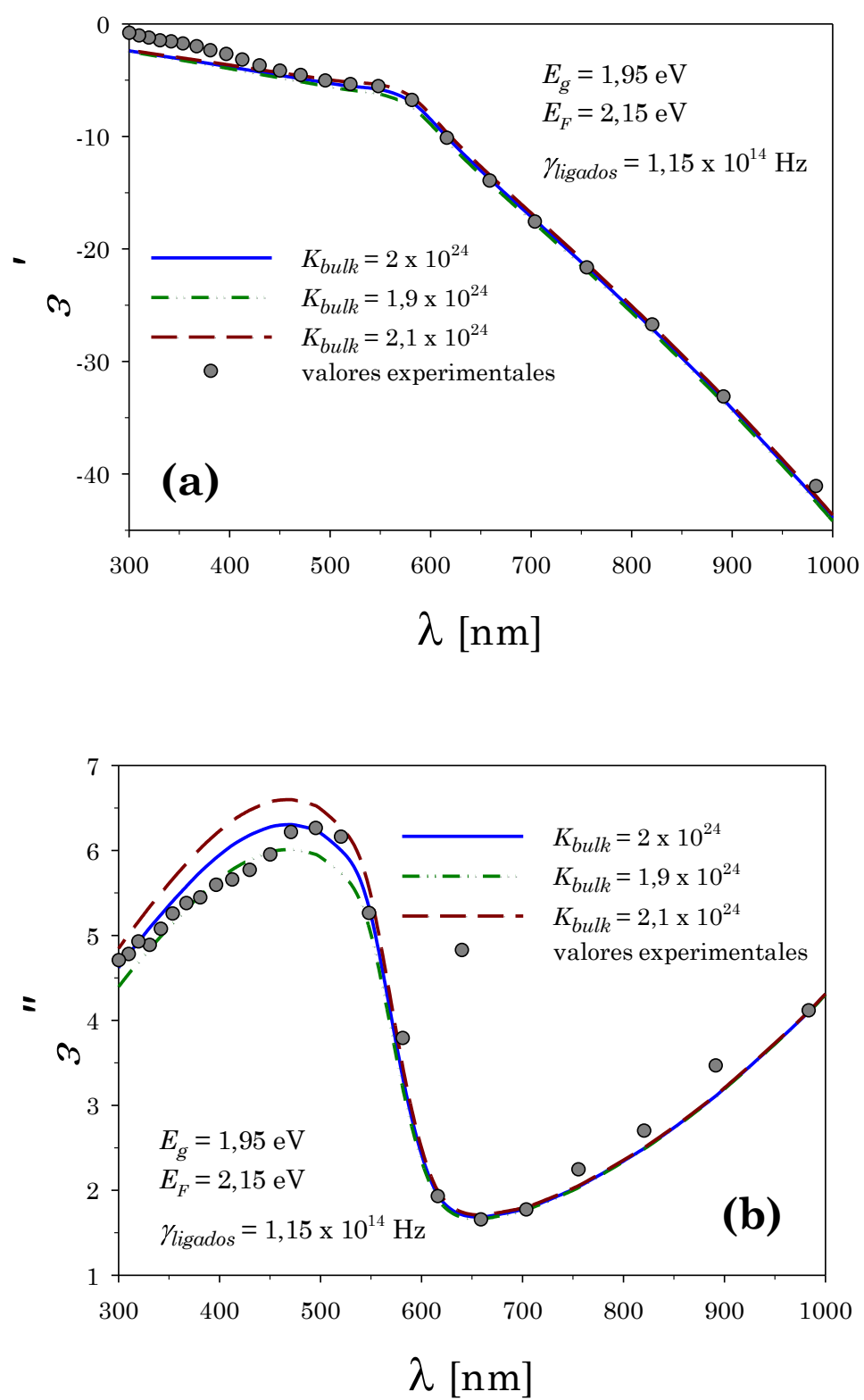

Figura 6.8: Influencia de $K_{b u l k}$ en la función dieléctrica compleja bulk del cobre: (a) parte real y (b) imaginaria. La influencia en la parte imaginaria es más notable entre 300 y $530 \mathrm{~nm}$. 


\section{- Plata}

Similarmente a lo realizado para el caso del cobre, en la Figura 6.9 se muestran por una parte las componentes real (a) e imaginaria (b) de las contribuciones de los electrones libres (ecuación (3.21)) y ligados (ecuación (3.37)) calculadas por separado para el caso de la plata. Asimismo se representa la suma teórica de ambas contribuciones (línea sólida), que corresponde al mejor ajuste de los valores experimentales de plata macroscópica (círculos) tomados de Johnson y Christy. Utilizando como parámetros de ajuste $E_{g}, E_{F}, \gamma_{\text {ligados }}$ y $K_{\text {bulk }}$, fue posible lograr un muy buen acuerdo entre los valores calculados teóricamente y los datos experimentales.

En la Figura 6.9 (b) se observa un comportamiento dominante de la contribución de los electrones ligados sobre la contribución de los electrones libres para longitudes de onda por debajo de $400 \mathrm{~nm}$, mientras que para longitudes de onda por encima de $400 \mathrm{~nm}$ la contribución que comienza a dominar es la de los electrones libres.

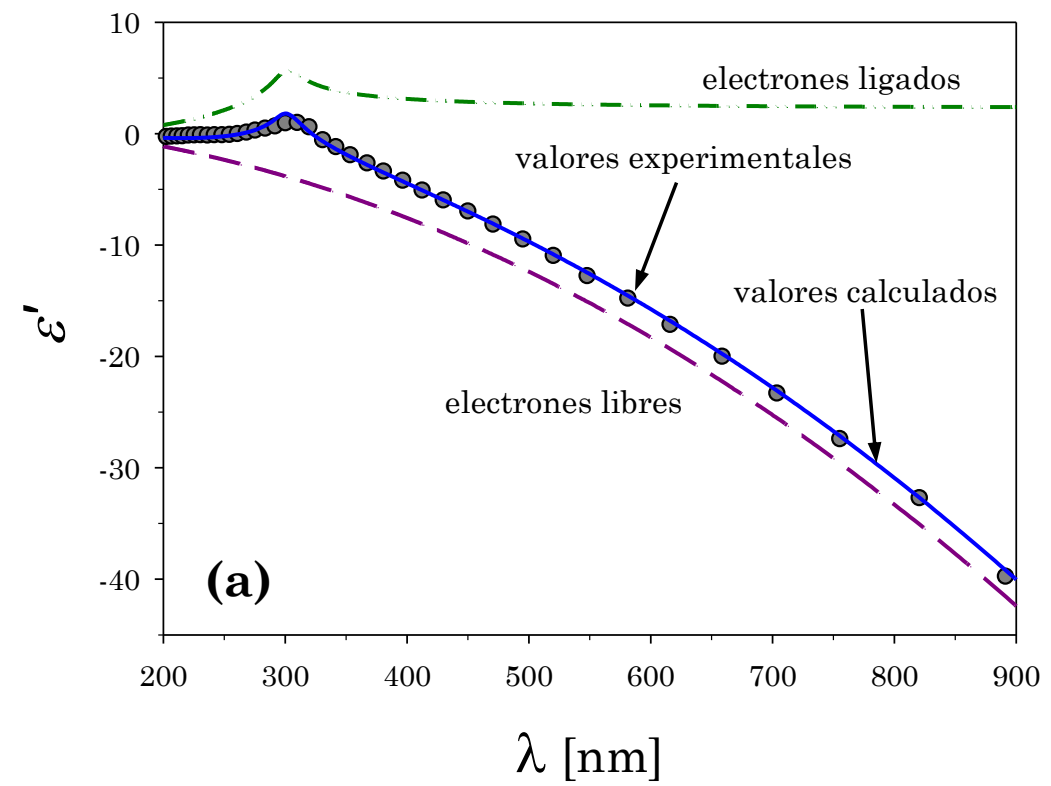




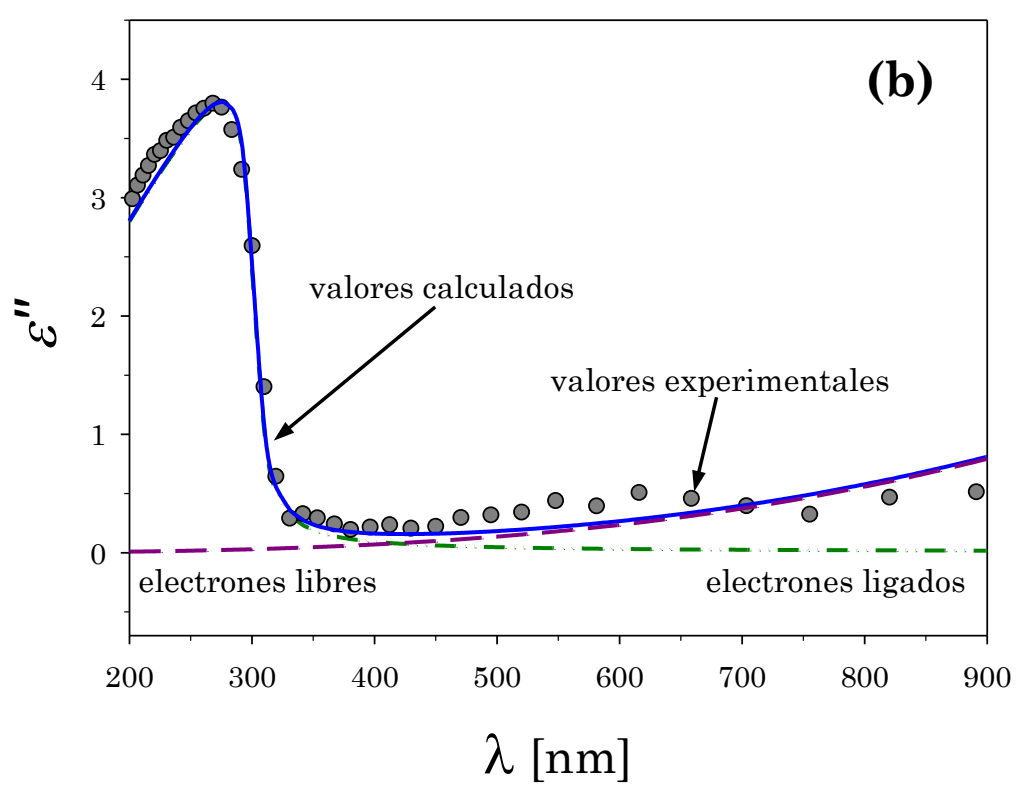

Figura 6.9: Contribuciones de los electrones libres y ligados a la función dieléctrica compleja de plata, calculadas con las ecuaciones (3.21) y (3.37). Los valores teóricos de las partes real (a) e imaginaria (b) de la función dieléctrica bulk de plata (ecuación (3.15)) son comparados con los valores experimentales (círculos) tomados de Johnson y Christy. Los parámetros usados y determinados están dados en la Tabla 6.2.

En la Tabla 6.2 se resumen los parámetros bibliográficos usados para el ajuste y los determinados en este trabajo de Tesis. Utilizando el modelo de la función dieléctrica explicado en la Sección 3.3, se han determinado por primera vez los parámetros microscópicos $K_{\text {bulk }}$ y $\gamma_{\text {ligados }}$ del modelo de electrones ligados [Santillán et al. 2013b]. A través de este procedimiento de ajuste espectral, se obtuvieron también los valores de $E_{F}$ y $E_{g}$ que coinciden, dentro de los errores experimentales, con los informados por otros autores y con otros métodos [Fox 2001], lo cual permite otorgar confiabilidad al método utilizado en esta Tesis, como así también a la determinación de los valores obtenidos de $K_{\text {bulk }}$ y $\gamma_{\text {ligados }}$. 
Tabla 6.2: Parámetros de la plata utilizados y determinados en este trabajo

\begin{tabular}{|c|c|c|c|}
\hline Parámetros de Ag & Símbolos & Valores & Referencias \\
\hline Frecuencia de plasma & $\omega_{p}$ & $13,8 \times 10^{15} \mathrm{~Hz}$ & $\begin{array}{l}\text { [Johnson and } \\
\text { Christy 1972] }\end{array}$ \\
\hline $\begin{array}{l}\text { Constante de amortiguamiento } \\
\text { para los electrones libres }\end{array}$ & $\gamma_{\text {libres }}$ & $2,7 \times 10^{13} \mathrm{~Hz}$ & $\begin{array}{l}\text { [Johnson and } \\
\text { Christy 1972] }\end{array}$ \\
\hline Velocidad de Fermi & $v_{F}$ & $14,1 \times 10^{14} \mathrm{~nm} / \mathrm{s}$ & [Kittel 2007] \\
\hline $\begin{array}{l}\text { Coeficiente de la contribución } \\
\text { del electrón ligado }\end{array}$ & $K_{\text {bulk }}$ & $2 \times 10^{24}$ & $\begin{array}{l}\text { [Santillán et al. } \\
\text { 2013b] }\end{array}$ \\
\hline Energía del gap & $E_{g}$ & $1,91 \mathrm{eV}$ & $\begin{array}{l}\text { [Santillán et al. } \\
\text { 2013b] }\end{array}$ \\
\hline Energía de Fermi & $E_{F}$ & $4,12 \mathrm{eV}$ & $\begin{array}{l}\text { [Santillán et al. } \\
\text { 2013b] }\end{array}$ \\
\hline $\begin{array}{l}\text { Constante de amortiguamiento } \\
\text { para los electrones ligados }\end{array}$ & $\gamma_{\text {ligados }}$ & $1,5 \times 10^{14} \mathrm{~Hz}$ & $\begin{array}{l}\text { [Santillán et al. } \\
\text { 2013b] }\end{array}$ \\
\hline
\end{tabular}

Utilizando los valores de los parámetros $K_{\text {bulk }}, E_{g}, E_{F}$ y $\gamma_{\text {ligados }}$, mostrados en la Tabla 6.2, determinados para el bulk, se realiza un análisis por separado de la contribución de los electrones libres y ligados a la función dieléctrica para los casos de radios nanométricos y subnanométricos. Más adelante se estudiará la influencia de estas funciones dieléctricas dependientes del tamaño en los espectros de extinción.

La Figura 6.10 representa el comportamiento de la componente real (a) e imaginaria (b) de la contribución de los electrones libres a la función dieléctrica de plata, para distintos radios de partículas usando las ecuaciones (3.29) y (3.30). En la Figura 6.10 (a) se puede ver que la 
componente real de los electrones libres es muy sensible para $a<2 \mathrm{~nm}$, como también sucede para el cobre. Mientras que en el rango de 2 a $10 \mathrm{~nm}$ es menos sensible y casi coincidente con la curva que corresponde a bulk para un radio de $10 \mathrm{~nm}$.
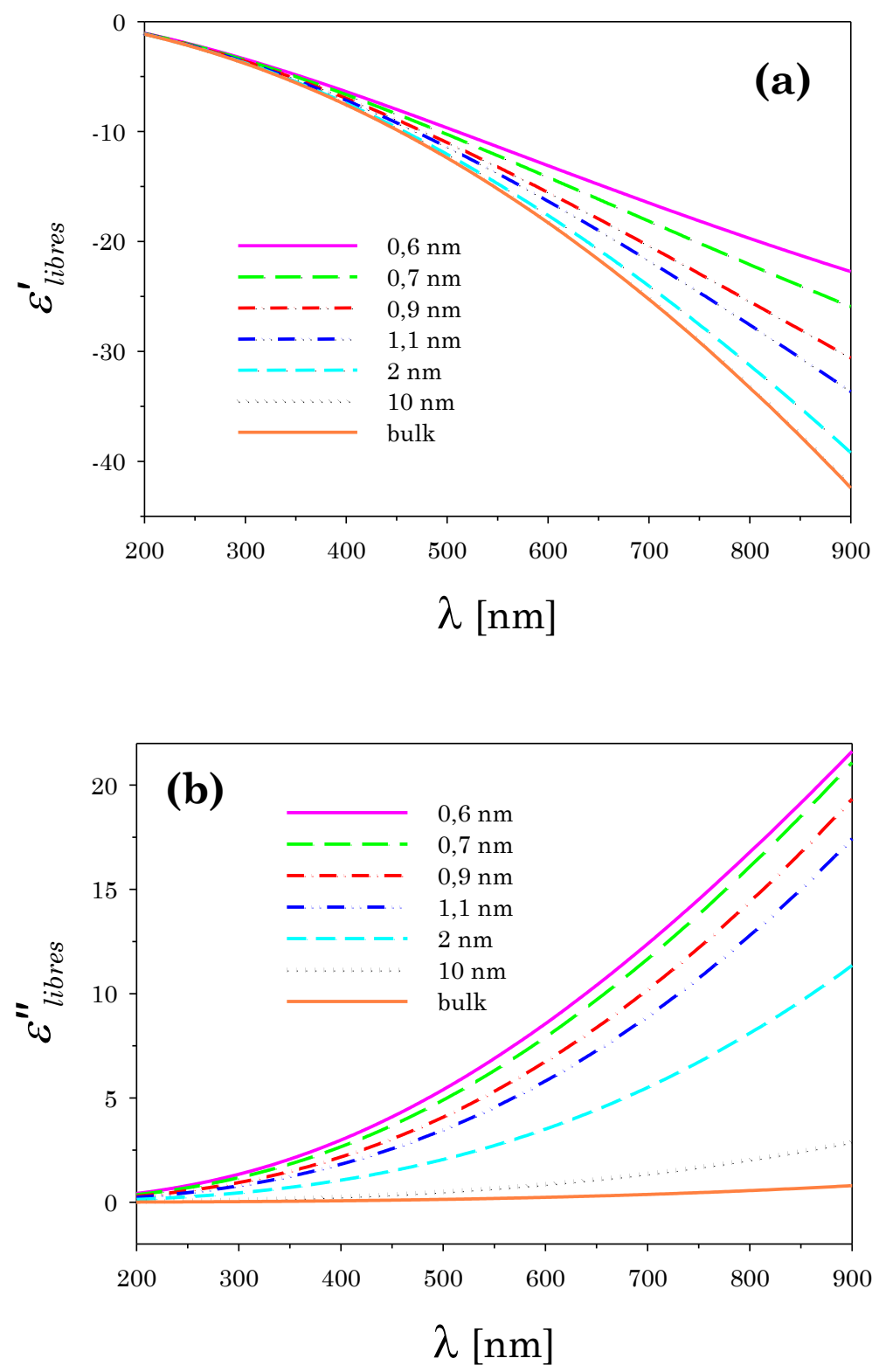

Figura 6.10: Contribución de los electrones libres a la función dieléctrica de plata en función de la longitud de onda para distintos radios. (a) Parte real y (b) imaginaria, calculadas con las ecuaciones (3.29) y (3.30), respectivamente. 
Por otro lado, en la componente imaginaria (Figura 6.10 (b)) se observan grandes variaciones para radios entre 2 y $20 \mathrm{~nm}$, aproximadamente, mientras se aprecia menos sensibilidad al cambio de radio para $a>20 \mathrm{~nm}$ y para $a<0,7 \mathrm{~nm}$.

Las componentes real (a) e imaginaria (b) de la contribución de los electrones ligados a la función dieléctrica dependiente del tamaño están representadas en la Figuras 6.11, para distintos valores de radio usando la expresión (3.37) teniendo en cuenta la corrección por tamaño $K_{t}$.

En la Figura 6.11 (a) se observa que las curvas son muy sensibles para $a<1,5 \mathrm{~nm}$ para longitudes de onda por encima de $300 \mathrm{~nm}$ aproximadamente. La corrección por tamaño de los electrones ligados en la Figura 6.11 (b) se hace muy notable para radios $a<1,5 \mathrm{~nm}$ para longitudes de onda por debajo de $300 \mathrm{~nm}$ aproximadamente, observándose que las curvas que corresponden a radios entre 2 y $10 \mathrm{~nm}$, están superpuestas para longitudes de onda por encima de $300 \mathrm{~nm}$. Resumiendo, la corrección de los electrones ligados tiene mayor influencia por debajo de $2 \mathrm{~nm}$ y en diferentes rangos espectrales, según se trate de la parte real o de la parte imaginaria. Para radios $a \geq 2 \mathrm{~nm}$, esta corrección se hace despreciable.

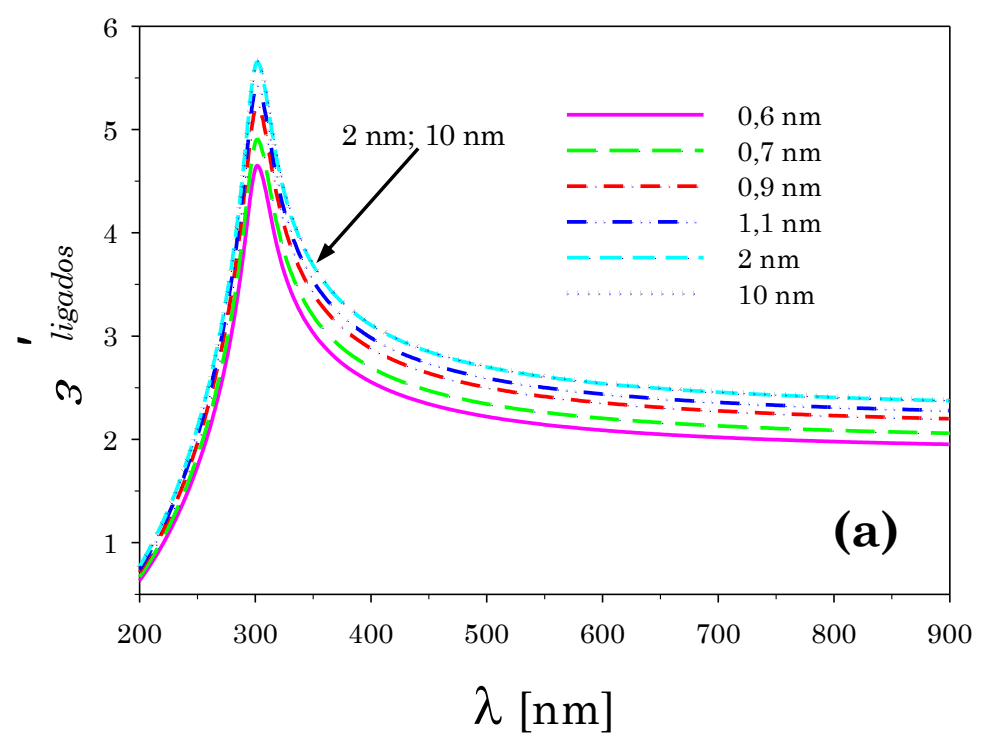




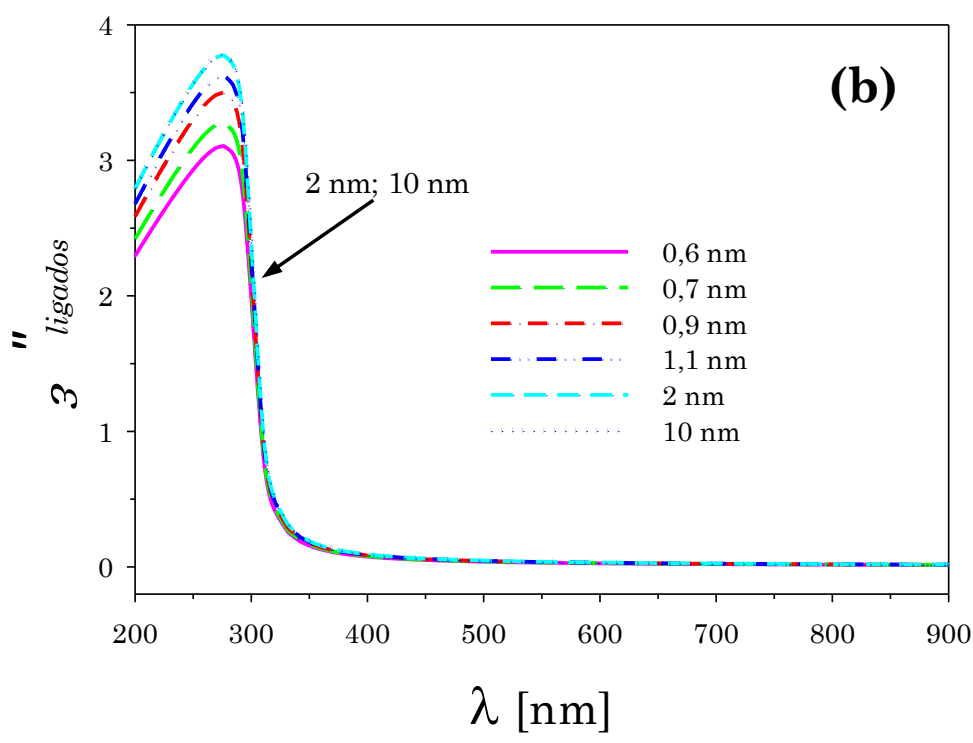

Figura 6.11: Contribución de los electrones ligados a la función dieléctrica de plata en función de la longitud de onda para diferentes radios. (a) Componente real y (b) componente imaginaria, calculadas con la ecuación (3.37) teniendo en cuenta la corrección por tamaño.

La Figura 6.12 muestra la parte real (a) e imaginaria (b) de la función dieléctrica total (ecuación (3.15)) como función de la longitud de onda para distintos tamaños de partículas de Ag.

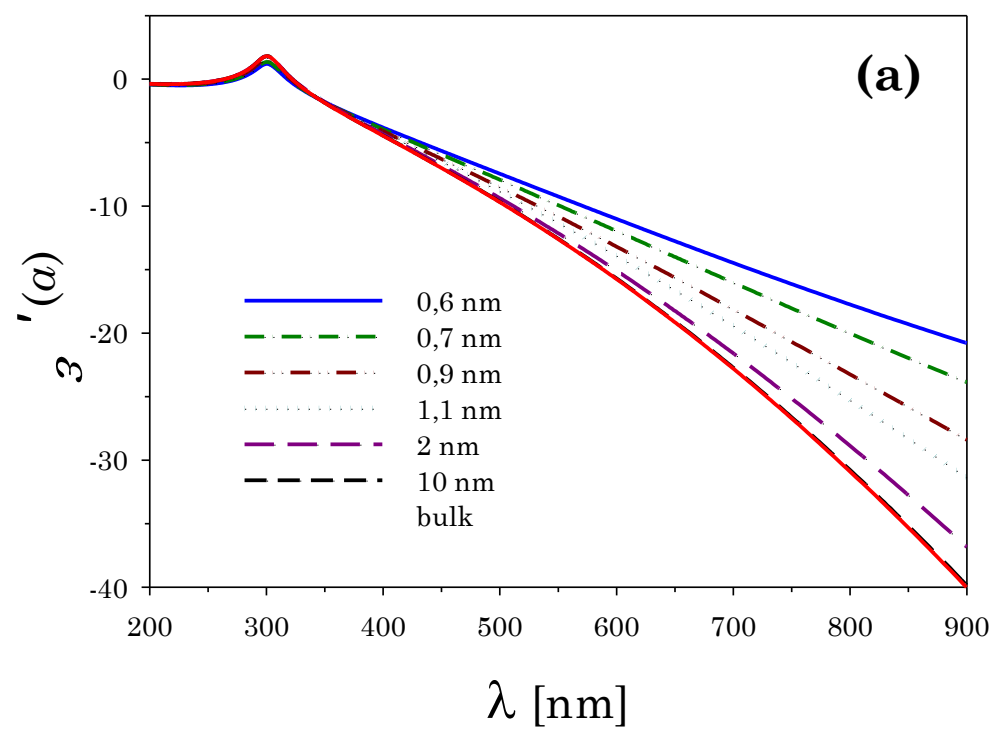




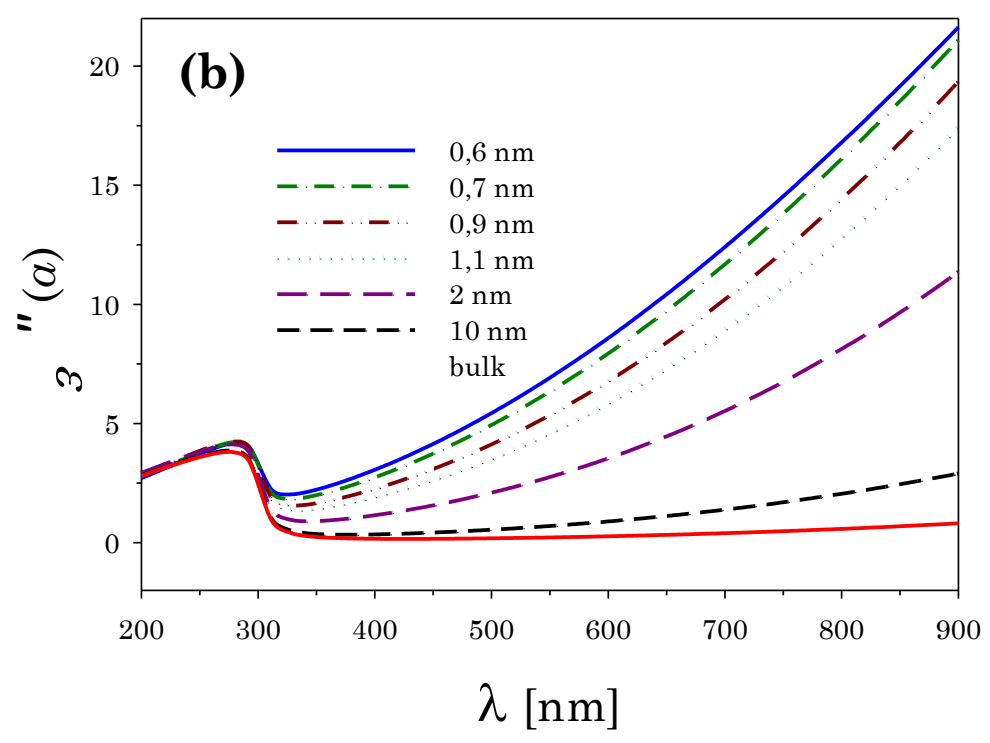

Figura 6.12: (a) Parte real y (b) parte imaginaria de la función dieléctrica completa de plata. La curva roja representa los datos experimentales tomados de Johnson y Christy. Las curvas restantes representan la corrección por tamaño para partículas de 0,6 a $10 \mathrm{~nm}$ de radio.

Se puede ver en la Figura 6.12 (a) que la componente real de la función dieléctrica total presenta importantes variaciones con el tamaño para longitudes de onda $\lambda \geq 350 \mathrm{~nm}$. En la Figura 6.12 (b) se observa una marcada dependencia con el tamaño de la componente imaginaria de la función dieléctrica total para longitudes de onda $\lambda \geq 300 \mathrm{~nm}$.

En las Figuras 6.13 a 6.16 , se representan las variaciones en la función dieléctrica compleja bulk debido a los pequeños cambios en los parámetros: $E_{g}, E_{F}, \gamma_{\text {ligados }}$, y $K_{\text {bulk }}$, respectivamente.

En la Figura 6.13 se muestra la influencia de $E_{g}$ en la función dieléctrica compleja. Si bien la parte real (Figura 6.13 (a)) no es tan sensible a los cambios en $E_{g}$, se aprecia una pequeña diferencia en las curvas calculadas para la parte imaginaria (Figura 6.13 (b)), para longitudes de onda $\lambda \leq 290 \mathrm{~nm}$. 

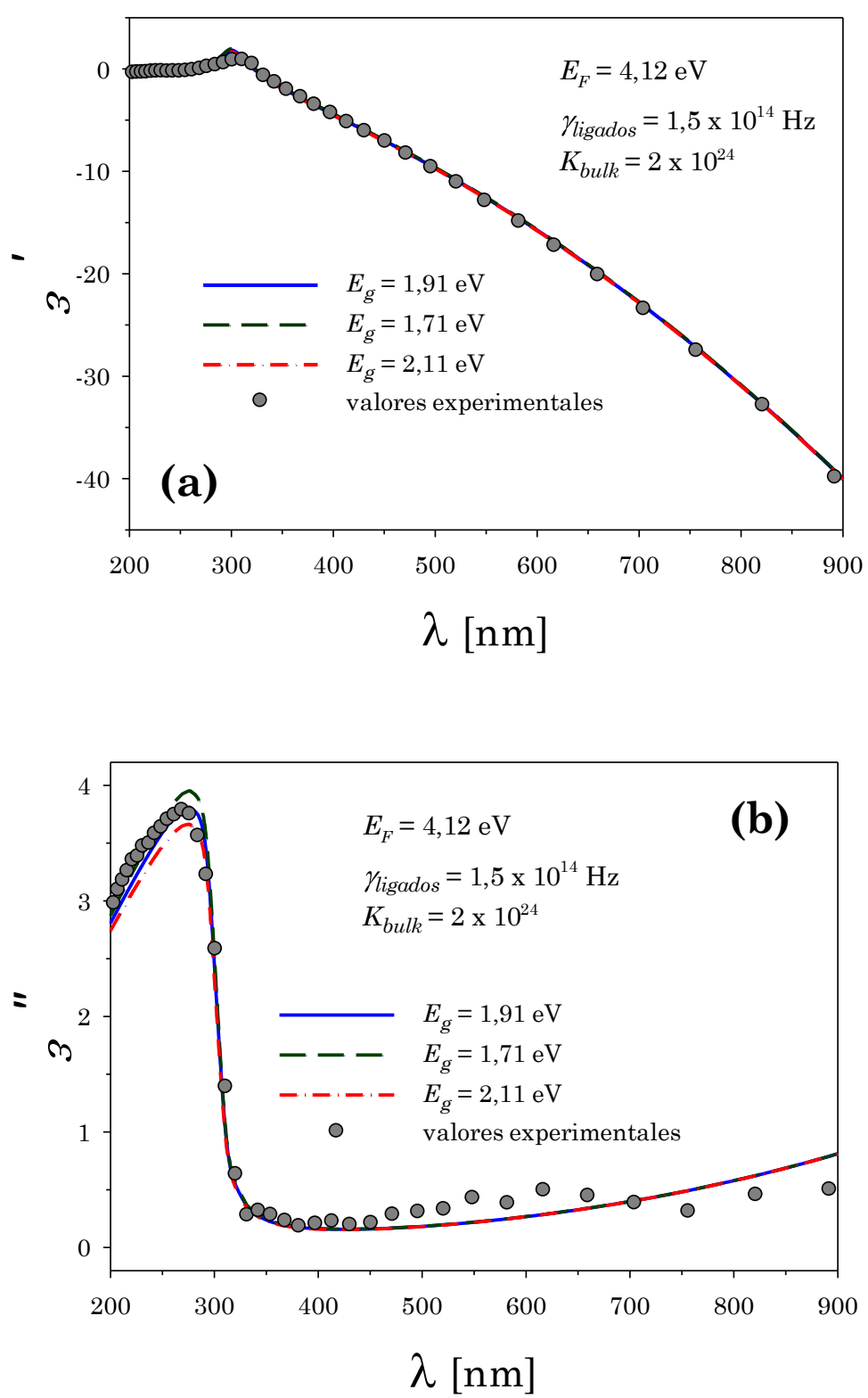

Figura 6.13: Influencia de $E_{g}$ en la función dieléctrica compleja bulk de plata: (a) componente real y (b) componente imaginaria. La influencia en la parte imaginaria es apreciable para longitudes de onda por debajo de $290 \mathrm{~nm}$.

En la Figura 6.14 se observa el comportamiento de $E_{F}$ en la función dieléctrica compleja bulk de plata. Se puede notar que para pequeñas variaciones en el valor de $E_{F}$, la parte real (Figura 6.14 (a)) sigue siendo 
invariable. La parte imaginaria en la Figura 6.14 (b) presenta cambios notorios para longitudes de onda que están entre 270 y $340 \mathrm{~nm}$, siendo este intervalo diferente de aquel en el que la influencia de $E_{g}$ se hace notable.
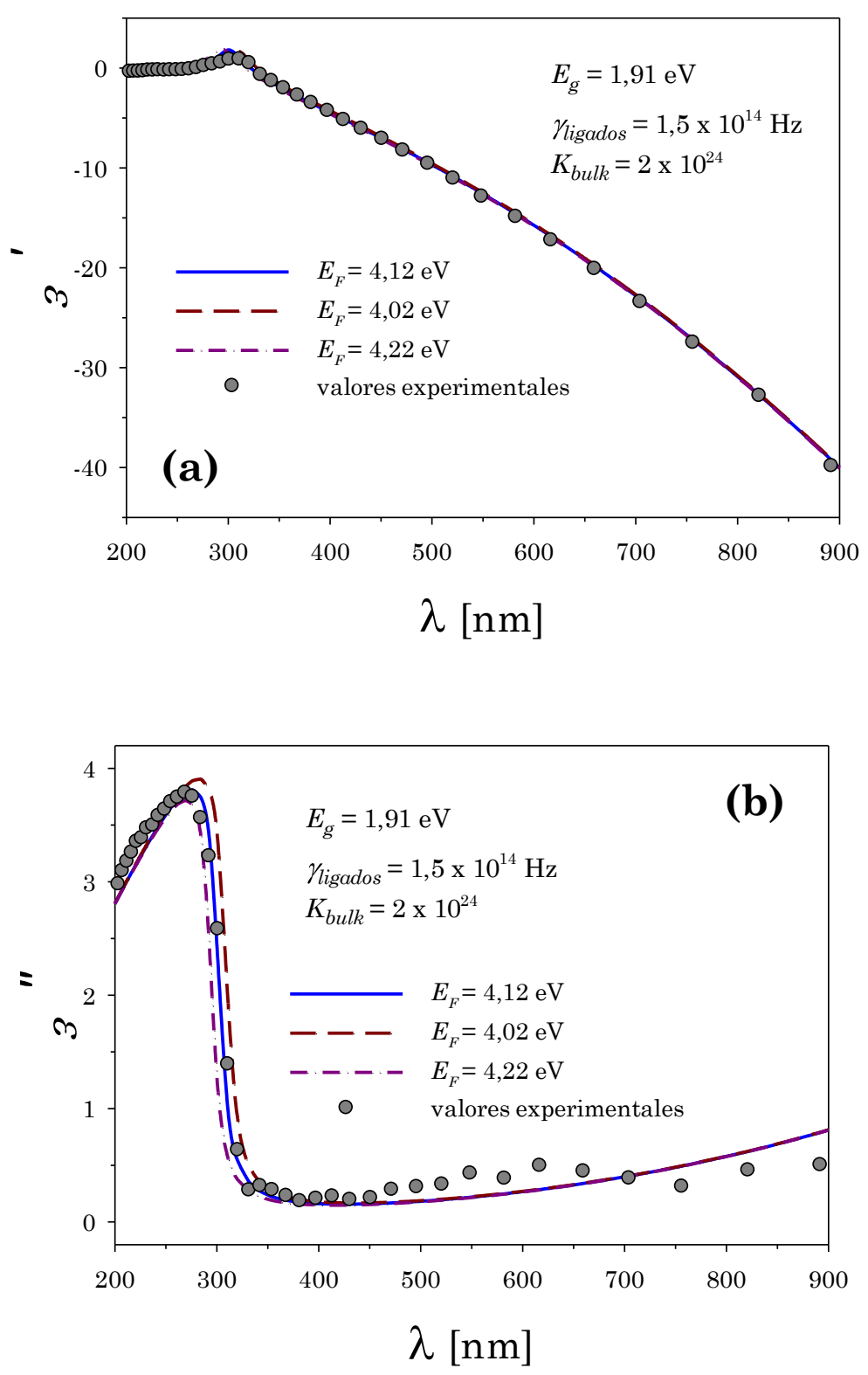

Figura 6.14: Influencia de $E_{F}$ en la función dieléctrica compleja bulk de plata: (a) parte real y (b) imaginaria. La influencia en la parte imaginaria es más notable entre 270 y $340 \mathrm{~nm}$. 
La Figura 6.15 representa la influencia de las pequeñas modificaciones de $\gamma_{\text {ligados }}$ sobre las componentes real (a) e imaginaria (b) de la función dieléctrica bulk de plata.
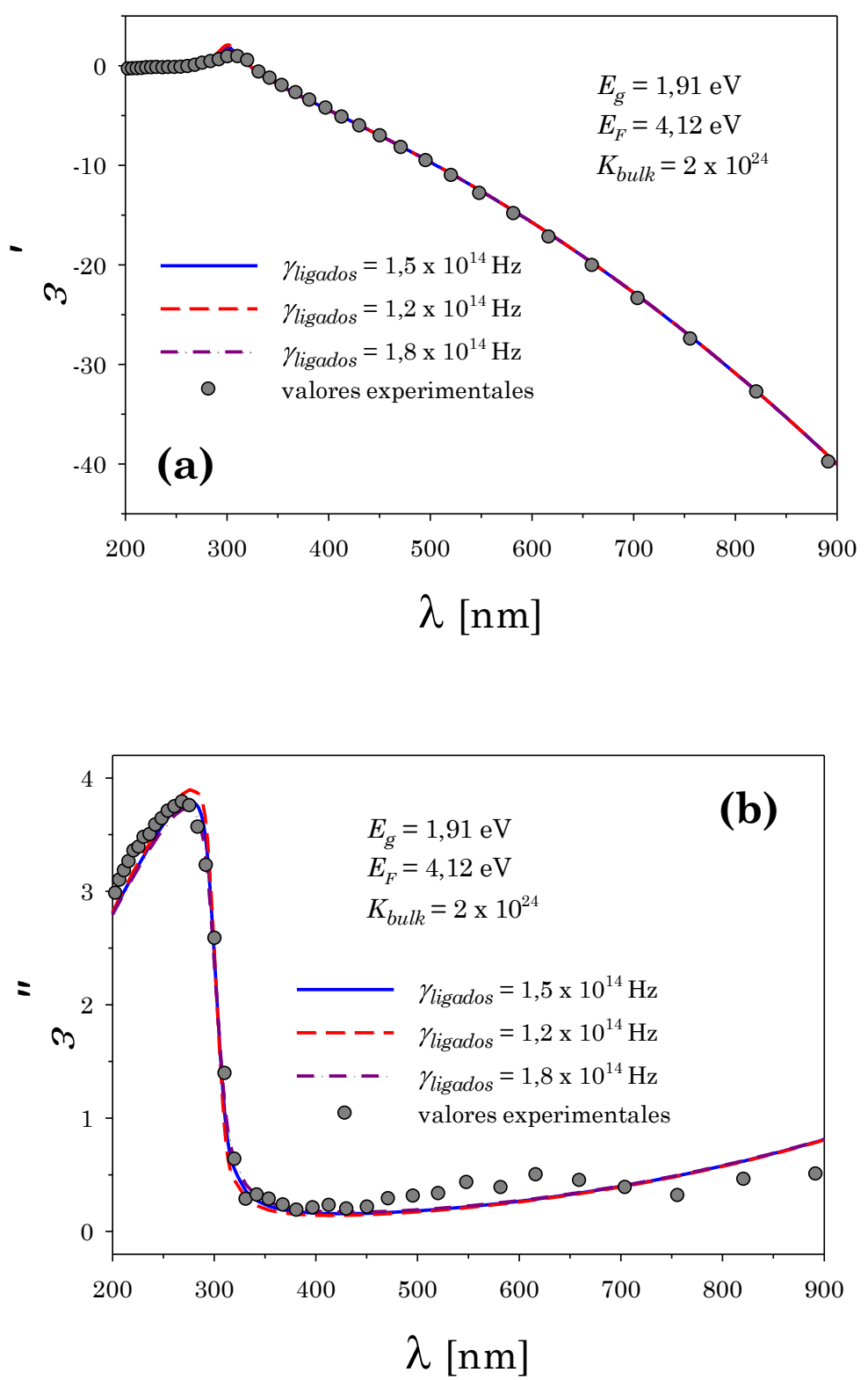

Figura 6.15: Influencia de $\gamma_{\text {ligados }}$ en la función dieléctrica compleja bulk de plata: (a) parte real y (b) imaginaria. Variaciones casi imperceptibles se observan en la parte imaginaria para longitudes de onda de 280 y $340 \mathrm{~nm}$. 
Aquí nuevamente, mientras que en la parte real (Figura 6.15 (a)) no existen cambios, la parte imaginaria (Figura 6.15 (b)) presenta variaciones casi imperceptibles alrededor de 280 y $340 \mathrm{~nm}$ (pico y valle).
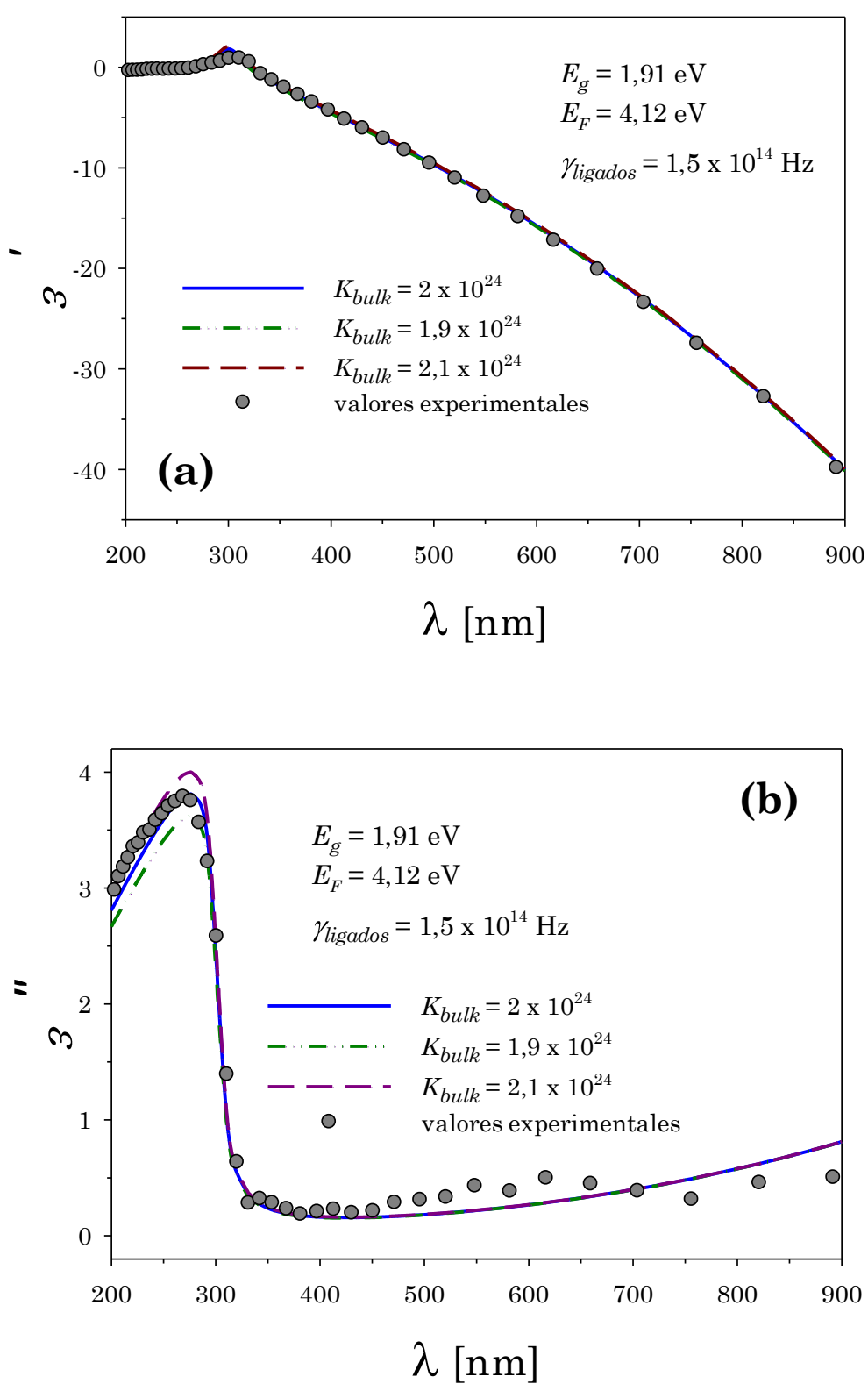

Figura 6.16: Influencia de $K_{\text {bulk }}$ en la función dieléctrica compleja bulk de plata: (a) componente real y (b) imaginaria. La influencia en la parte imaginaria es más notable por debajo de $290 \mathrm{~nm}$. 
Por último, en la Figura 6.16 se muestra la influencia de $K_{b u l k}$ en la función dieléctrica compleja bulk de plata. En este caso se puede observar que para pequeños cambios en el valor de $K_{b u l k}$, la parte imaginaria (Figura 6.16 (b)) tiene cambios visibles por debajo de $290 \mathrm{~nm}$. En la parte real (Figura 6.16 (a)) los cambios no son notables.

Analizando la influencia que los parámetros $K_{b u l k}, E_{g}, E_{F}$ y $\gamma_{\text {ligados }}$ ejercen sobre la función dieléctrica compleja de bulk de plata, fue posible ajustar exitosamente la función dieléctrica experimental tomada de los datos de Johnson y Christy.

\subsubsection{Análisis del comportamiento de los espectros de extinción}

Una vez obtenidos los parámetros relacionados con las contribuciones de los electrones libres y ligados mencionados en las Tablas 6.1 y 6.2 para cobre y plata respectivamente, y luego de analizar el comportamiento de la función dieléctrica con el tamaño para ambos metales en un rango de radios nanométricos y subnanométricos, fue posible, estudiar teóricamente la influencia de aquélla en los espectros de extinción.

El cálculo teórico del coeficiente de extinción de partículas de estructuras simples y núcleo-recubrimiento de diámetros nanométricos y subnanométricos se realizó a través de la aproximación cuasi-estática (ecuación (2.33)). Teniendo en cuenta este análisis, fue posible ajustar los espectros de extinción experimentales de partículas esféricas nanométricas y subnanométricas generadas por ablación láser ultrarrápida de un blanco sólido ( $\mathrm{Cu}$ o Ag) en agua como se ha descripto en el Capítulo 5. 
Dado que durante el proceso de ablación existen condiciones de alta temperatura y presión en el plasma generado en la pluma, los procesos de oxidación ocurren muy rápido sobre las partículas nanométricas y subnanométricas de cobre y plata formadas, y existe una gran probabilidad de que se generen partículas de cobre-óxido de cobre y plata-óxido de plata. Por lo tanto la expresión de la polarizabilidad que se utilizó para ajustar estos espectros corresponde a la de esferas core-shell (ecuación (2.34)). Para los ajustes antes mencionados se ha utilizado el coeficiente de extinción $Q_{\text {ext }}$ que está relacionado con la sección eficaz de extinción a través de la siguiente expresión:

$$
Q_{e x t}=\frac{C_{e x t}}{2 \pi a_{2}^{2}}
$$

\section{- Cobre}

La Figura 6.17 (a) muestra el coeficiente de extinción en función de la longitud de onda de un partícula core-shell de cobre-óxido de cobre $(\mathrm{Cu}$ $\mathrm{Cu}_{2} \mathrm{O}$ ) para un núcleo metálico de radio subnanométrico $a_{1}=0,7 \mathrm{~nm}$ cubierto por una fina capa de $\mathrm{Cu}_{2} \mathrm{O}\left(a_{2}-a_{1}=50 \% a_{1}\right)$ en agua, calculado con y sin corrección por tamaño de los electrones ligados. Los datos de la función dieléctrica experimental del óxido de cobre fueron tomados de Palik [Palik 1985] y se muestran en el recuadro de esta figura. Se puede observar que la posición del pico está alrededor de $650 \mathrm{~nm}$ para ambos espectros, pero las diferencias son más perceptibles para longitudes de onda inferiores a $650 \mathrm{~nm}$, donde la influencia de la parte imaginaria de los electrones ligados es más importante. La corrección de los electrones ligados suaviza el contraste $\left(I_{\text {máx }}-I_{\text {min }}\right) / I_{\text {máx }}$ en el rango mencionado, mientras que para longitudes de onda grandes los espectros coinciden. 

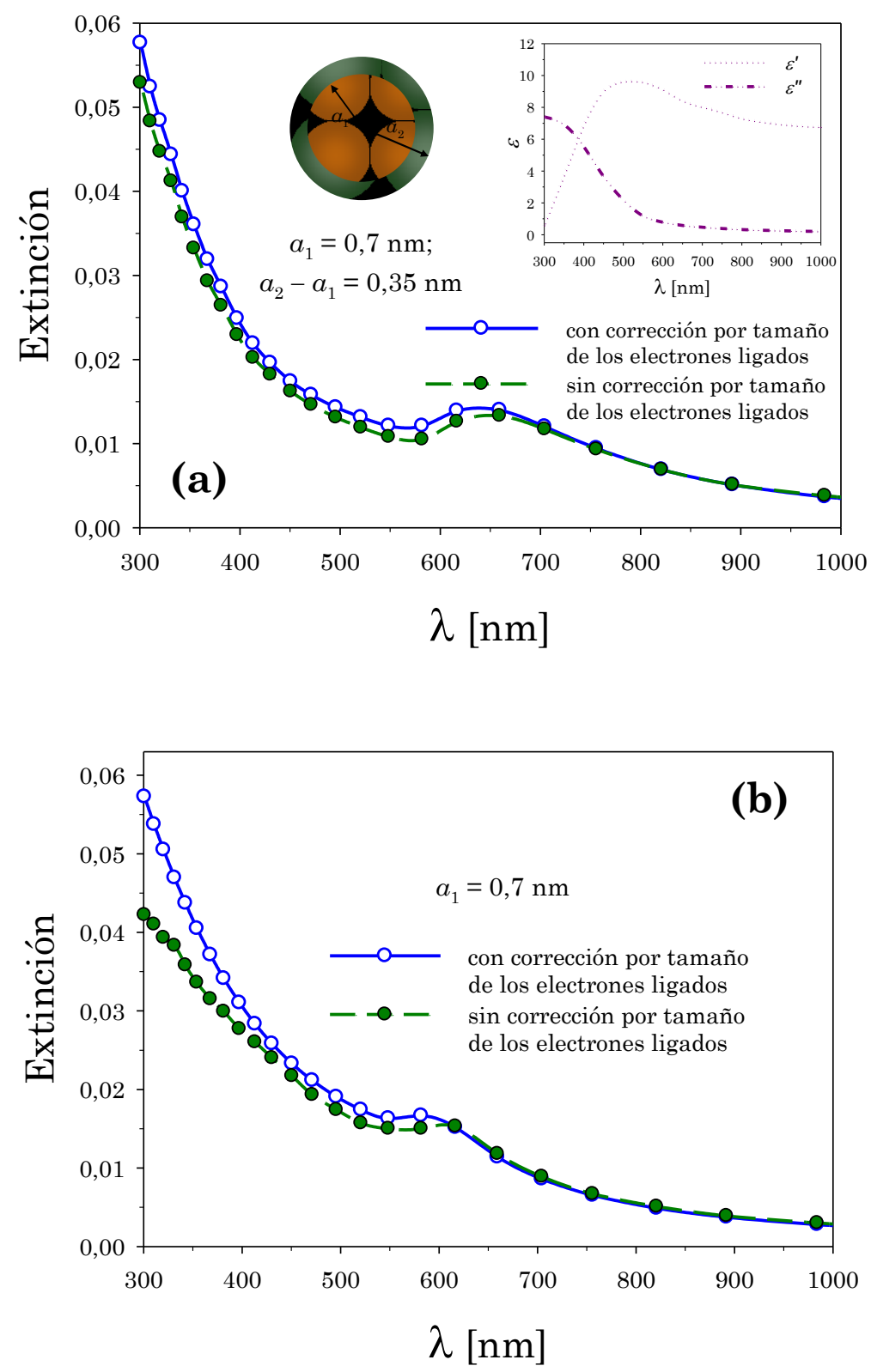

Figura 6.17: Coeficiente de extinción para una nanopartícula de $\mathrm{Cu}$ de estructura (a) core-shell y (b) simple, con y sin corrección por tamaño de los electrones ligados. La diferencia en el espectro es más notable para longitudes de onda por debajo del pico del plasmón, donde la influencia de los electrones ligados es más importante.

En la Figura 6.17 (b) se muestra el coeficiente de extinción de una partícula subnanométrica de cobre de estructura simple, que es un caso especial de partículas core-shell donde $a_{2}=a_{1}$. Aquí, las diferencias entre 
los espectros son más evidentes para longitudes de onda por debajo de $600 \mathrm{~nm}$, y la resonancia del plasmón está desplazada hacia $650 \mathrm{~nm}$ (con shell de óxido) con respecto al pequeño pico ubicado en $600 \mathrm{~nm}$ sin shell.

Después de analizar el comportamiento del coeficiente de extinción para diferentes estructuras y tamaños de partículas nanométricas y subnanoméricas, se aplicaron los cálculos anteriores para ajustar espectros de extinción experimentales correspondientes a las suspensiones coloidales obtenidas previamente por ablación láser.

La Figura 6.18 muestra el mejor ajuste del espectro de extinción experimental normalizado correspondiente a una suspensión coloidal de nanopartículas de $\mathrm{Cu}-\mathrm{Cu}_{2} \mathrm{O}$ generadas en agua por ablación láser de pulsos de femtosegundo con una energía por pulso de $500 \mu \mathrm{J}$.

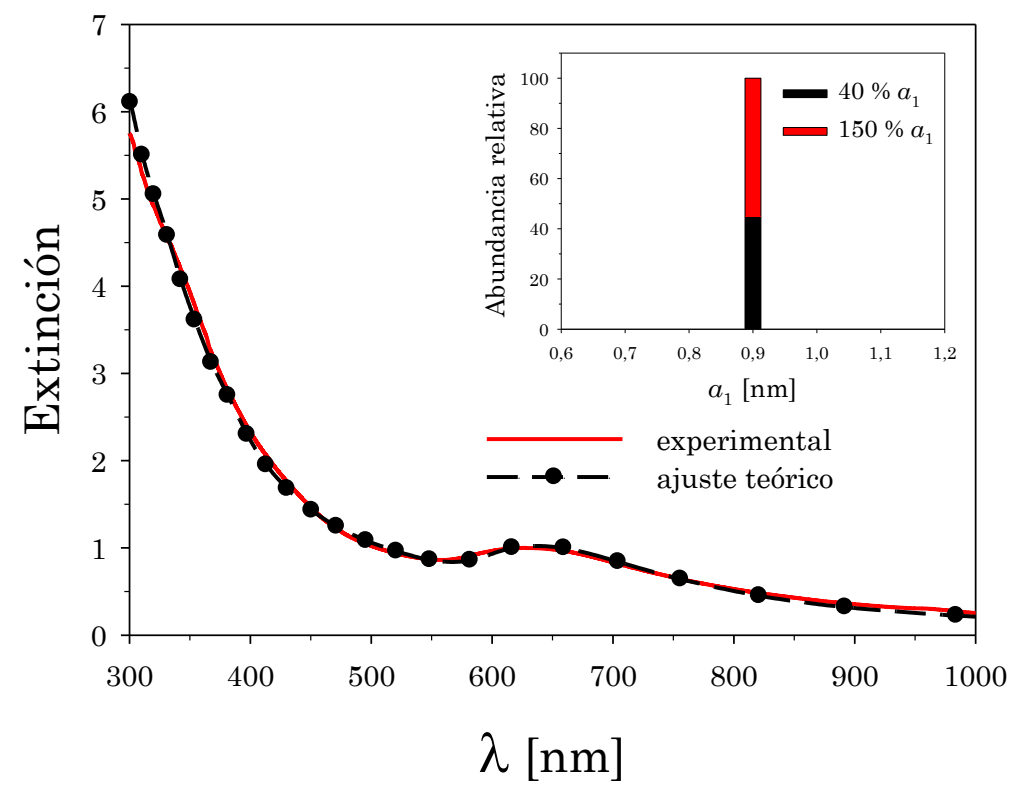

Figura 6.18: La línea roja corresponde al espectro de extinción experimental normalizado de nanopartículas de $\mathrm{Cu}-\mathrm{Cu}_{2} \mathrm{O}$ fabricadas en agua por ablación láser con una energía por pulso de $500 \mu \mathrm{J}$. La línea discontinua con círculos muestra el mejor ajuste calculado teóricamente con una contribución bimodal de nanopartículas de $\left.\mathrm{Cu}-\mathrm{Cu}_{2} \mathrm{O}: 1\right) \quad a_{1}=0,9 \mathrm{~nm}$ y $a_{2}-a_{1}=0,36 \mathrm{~nm}$ y 2$) a_{1}=0,9 \mathrm{~nm}$ y $a_{2}-a_{1}=1,35 \mathrm{~nm}$. La abundancia para la primera contribución es 0,45 y para la segunda es 0,55 [Santillán et al. 2012a, Santillán et al. 2012b]. 
Este ajuste se logró teniendo en cuenta una combinación lineal de dos tamaños de nanopartículas core-shell: una con $0,9 \mathrm{~nm}$ de radio del núcleo y $40 \% a_{1}$ de espesor del shell y otra del mismo radio del núcleo y un $150 \% a_{1}$ de espesor del shell. Los coeficientes de esta combinación, 0,45 y 0,55 respectivamente, dan la abundancia relativa óptima de las especies.

Los cálculos teóricos para núcleos de radios subnanométricos están en excelente acuerdo con los resultados experimentales obtenidos de suspensiones coloidales de tipo core-shell.

Es interesante señalar también que ninguna combinación de partículas nanométricas y subnanométricas de estructura simple podría ajustar todo el espectro. Este hecho se puede ver en la Figura 6.19 donde se representan los espectros normalizados de cada especie junto con el espectro experimental para su comparación.

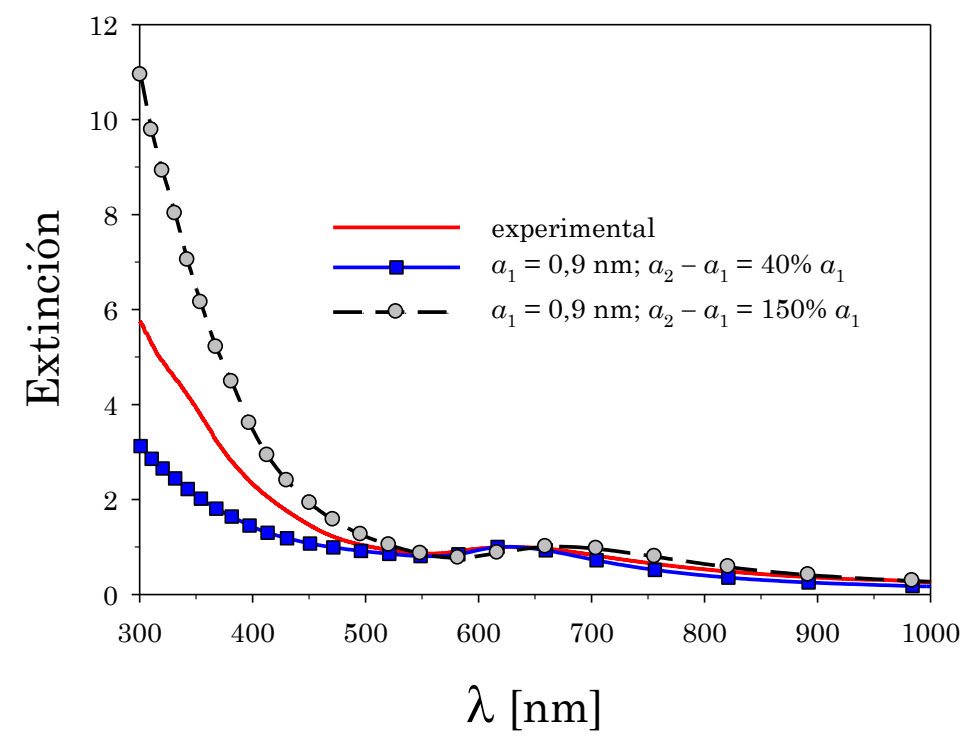

Figura 6.19: La línea roja corresponde al espectro de extinción experimental normalizado de nanopartículas de $\mathrm{Cu}-\mathrm{Cu}_{2} \mathrm{O}$ fabricadas en agua por ablación láser con una energía por pulso de $500 \mu \mathrm{J}$. La línea azul con cuadrados es el espectro de extinción calculado que corresponde a $a_{1}=0,9 \mathrm{~nm}$ y $a_{2}-a_{1}=0,36 \mathrm{~nm}$. La línea discontinua con círculos es el espectro de extinción calculado que corresponde a $a_{1}=0,9 \mathrm{~nm}$ y $a_{2}-a_{1}=1,35 \mathrm{~nm}$. 
Se puede observar que la curva que corresponde a una nanopartícula de $a_{1}=0,9 \mathrm{~nm}$ y $40 \%$ de espesor del shell ajusta correctamente la posición del pico del plasmón, pero existe un serio desacuerdo para longitudes de onda más cortas que $550 \mathrm{~nm}$. Mientras que la curva que corresponde a $150 \%$ de espesor del shell tiene su posición del pico del plasmón desplazado hacia el IR en $60 \mathrm{~nm}$ aproximadamente con respecto a la curva experimental, este cambio puede ser fácilmente medible con un espectrofotómetro comercial y no ajusta correctamente el espectro en el rango de longitudes de onda cortas.

Para analizar la sensibilidad del procedimiento de ajuste, la Figura 6.20 muestra el comportamiento de los espectros de extinción teóricos comparados con el experimental para diferentes valores de radios del núcleo y espesores del recubrimiento.

En la Figura 6.20 (a) se muestran los espectros correspondientes a la misma distribución óptima de espesores del shell que la utilizada en la Figura 6.18, pero para diferentes radios del núcleo. Se observa que, si bien los radios del núcleo son sólo $0,1 \mathrm{~nm}$ por encima y por debajo del valor óptimo $(0,9 \mathrm{~nm})$, existe una diferencia pequeña pero medible entre los espectros teóricos y experimental.

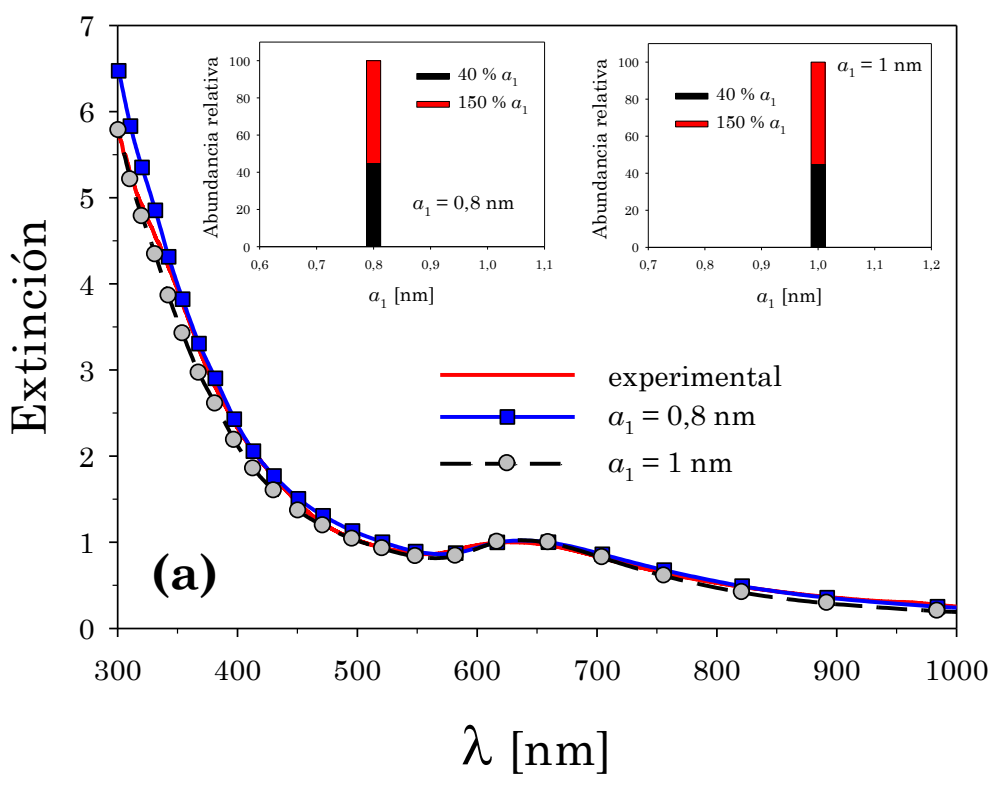




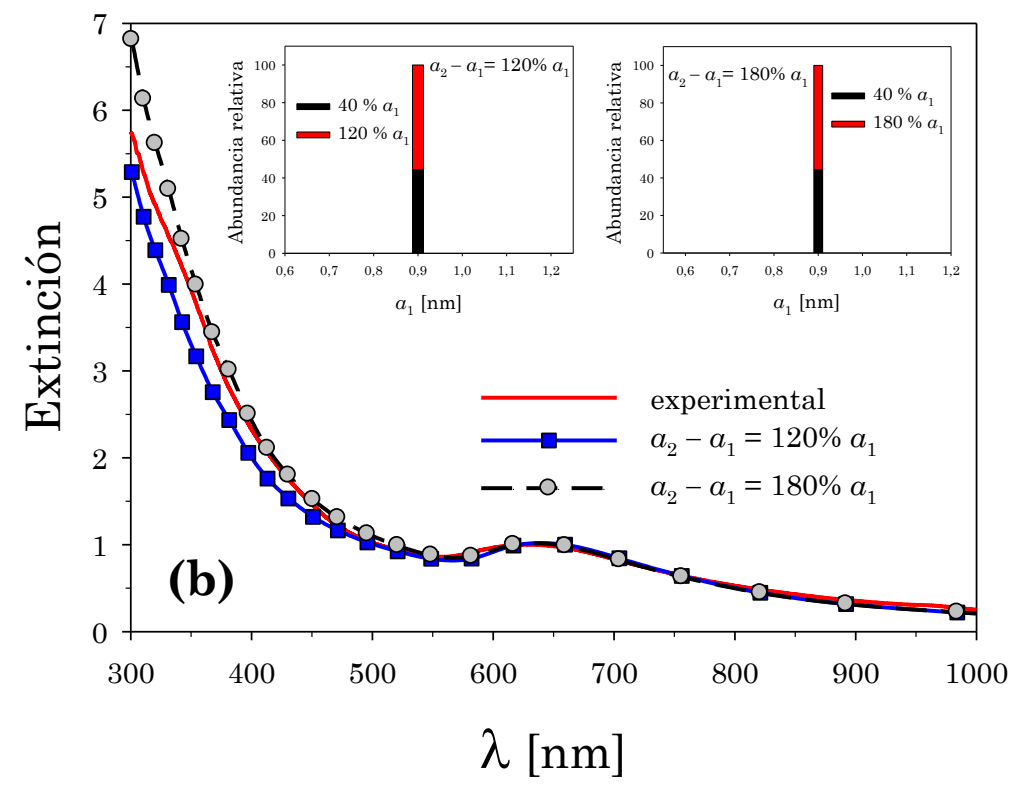

Figura 6.20: Espectros de extinción experimental y calculados, ambos normalizados, para: (a) diferentes radios del núcleo para la distribución de espesores óptima y (b) diferentes distribuciones de espesores del shell para el radio del núcleo óptimo.

En la Figura 6.20 (b) se representan los espectros correspondientes al mismo radio del núcleo óptimo pero con diferentes distribuciones en el espesor del shell. Manteniendo fijo el espesor de 40\% $a_{1}$ y haciendo variar el espesor más grande alrededor del valor óptimo de 150\% $a_{1}$ (entre 120\% $a_{1}$ y $180 \% a_{1}$ ), es posible observar que los espectros calculados muestran diferencias claras por debajo de $500 \mathrm{~nm}$, observándose una considerable sensibilidad en la respuesta de la extinción que hace posible que el ajuste final del espectro experimental se pueda lograr sólo con una única combinación tanto del radio del núcleo como del espesor del shell. Resumiendo, cambios en el radio del núcleo mayores que $\pm 0,1 \mathrm{~nm}$ o en el espesor del recubrimiento mayores que $\pm 20 \%$ hacen imposible el ajuste de la curva experimental para longitudes de onda más cortas que 500 nm. Este comportamiento demuestra la buena sensibilidad del procedimiento de ajuste. 
Luego de analizar el comportamiento de los espectros de extinción teóricos de estructura core-shell de cobre frente a variaciones de tamaño del núcleo y espesor del shell, es importante mencionar que este metal posee dos estados de oxidación que, dependiendo de la valencia con la que actúe, puede dar lugar al óxido cuproso $\left(\mathrm{Cu}_{2} \mathrm{O}\right)$ o al óxido cúprico $(\mathrm{CuO})$ alrededor del núcleo de $\mathrm{Cu}$. A continuación se analiza la influencia que el último tipo de óxido presenta en el espectro UV-visible, y se lo compara con el comportamiento que presenta el óxido $\mathrm{Cu}_{2} \mathrm{O}$.

La Figura 6.21 muestra el espectro experimental normalizado correspondiente a la suspensión coloidal generada con $500 \mu \mathrm{J}$ de energía por pulso junto con el mejor ajuste calculado con la estructura $\mathrm{Cu}-\mathrm{Cu}_{2} \mathrm{O}$ y un espectro calculado con la misma distribución de núcleos y espesores para una estructura de $\mathrm{Cu}-\mathrm{CuO}$.

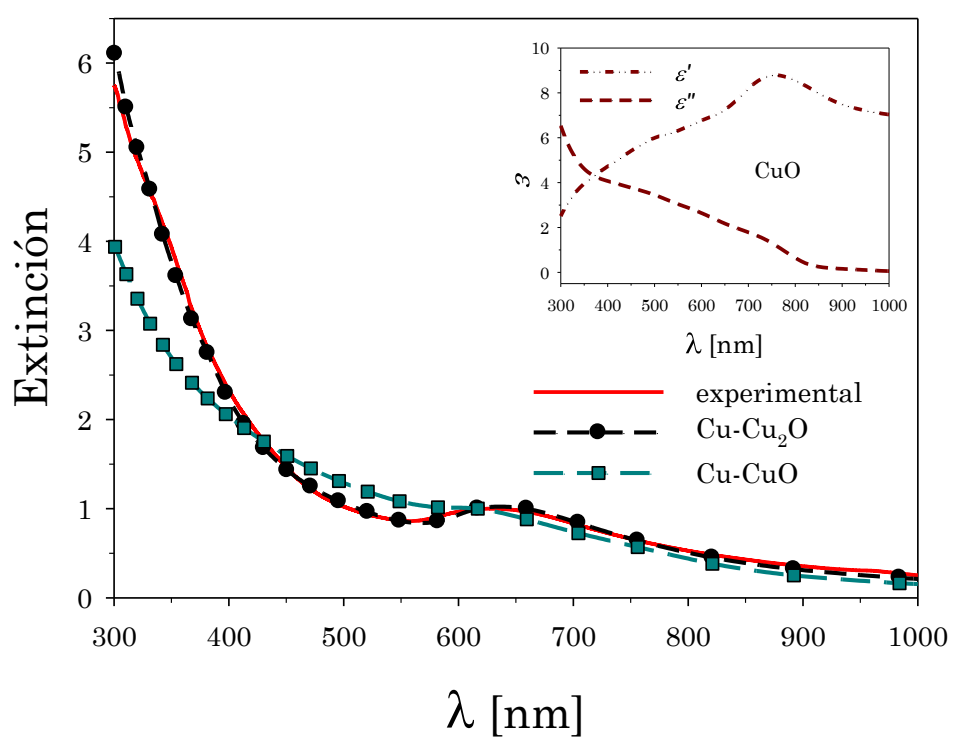

Figura 6.21: Espectros de extinción experimental y calculados, ambos normalizados, para estructuras de $\mathrm{Cu}-\mathrm{Cu}_{2} \mathrm{O}$ y $\mathrm{Cu}-\mathrm{CuO}$, utilizando la distribución de núcleo y espesores óptima. 
Se puede observar que el espectro de extinción teórico correspondiente a la estructura que contiene $\mathrm{CuO}$ como shell, presenta una resonancia plasmónica casi imperceptible (contraste nulo) y desplazada hacia el azul en aproximadamente $20 \mathrm{~nm}$ respecto a la estructura de $\mathrm{Cu}-\mathrm{Cu}_{2} \mathrm{O}$. Asimismo, se observa que la pendiente de la curva a $\lambda \leq 615 \mathrm{~nm}$ es menos pronunciada que la que presenta el espectro experimental. Este comportamiento se debe a las diferentes características espectrales de las componentes real e imaginaria del $\mathrm{CuO}$ (recuadro de la Figura 6.21), comparadas con las que presenta el $\mathrm{Cu}_{2} \mathrm{O}$ (recuadro de la Figura 6.17 (a)).

Para comparar los resultados de la determinación de tamaños obtenidos con el método de espectroscopía de extinción óptica, se realizó un análisis de microscopía de la misma muestra coloidal. La Figura 6.22 (a) muestra, como ejemplo, una imagen AFM de la muestra coloidal generada en agua usando ablación láser con una energía de $500 \mu \mathrm{J}$ por pulso. Las partículas individuales fueron resueltas con claridad por imágenes de AFM de un área de escaneo de 1,2 $\mu \mathrm{m}$ x 1,2 $\mu \mathrm{m}$ utilizando muestras de baja concentración. Se conoce que el radio de curvatura de la punta limita la resolución lateral, pero las mediciones de la altura tienen mayor resolución y se puede obtener el tamaño de nanopartículas con mayor precisión. Los perfiles de la altura de la línea 1 y la línea 2 en el panel superior de la Figura 6.22 (a) se muestran en el panel inferior. El escaneo de la primera línea en el rango de $0,9 \mu \mathrm{m}<x<1,1 \mu \mathrm{m}$ incluye una sola nanopartícula de 4,5 $\mathrm{nm}$ de altura (diámetro). Este tamaño coincide con los resultados obtenidos mediante espectroscopía de extinción óptica de la misma muestra, considerando una partícula core-shell de $\mathrm{Cu}-\mathrm{Cu}_{2} \mathrm{O}$ con un radio del núcleo de $0,9 \mathrm{~nm}$ y un espesor del shell de 1,35 nm. Por otra parte, la línea 2 de escaneo contiene una nanopartícula de 2,2 $\mathrm{nm}$ de altura, la cual muestra un buen acuerdo con el otro tamaño presente en la suspensión coloidal de $\mathrm{Cu}$ $\mathrm{Cu}_{2} \mathrm{O}$ de la Figura 6.18 y deriva del ajuste de su espectro de extinción. Para 
destacar la fiabilidad de estos resultados, las Figuras 6.22 (b) y (c) muestran la imagen y el perfil de la rugosidad del sustrato de mica sobre el cual se depositó la suspensión coloidal. La rugosidad promedio del sustrato de mica es de 0,07 nm (Figura 6.22 (c)) casi dos órdenes de magnitud menor que el tamaño de nanopartículas observadas, lo que garantiza una relación de contraste muy bueno entre las partículas y el fondo. Finalmente, la Figura 6.22 (d) muestra una imagen HRTEM de la misma suspensión coloidal, donde se pueden distinguir nanopartículas de estructura core-shell de $\mathrm{Cu}-\mathrm{Cu}_{2} \mathrm{O}$. El radio exterior de las nanopartículas se encuentra entre 1,5 y 2,5 $\mathrm{nm}$ estando de acuerdo con los valores determinados por microscopía AFM y espectroscopía de extinción óptica.

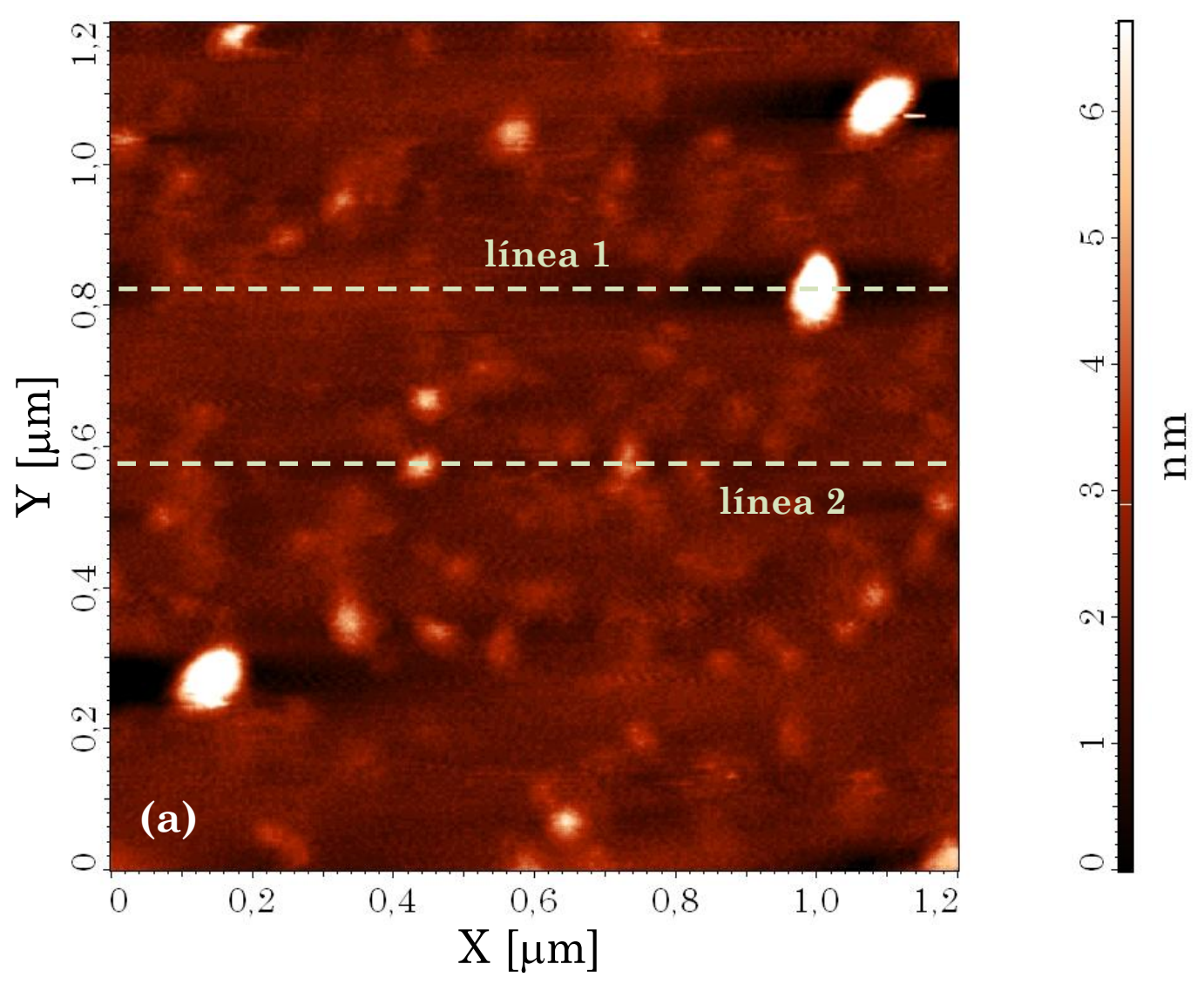



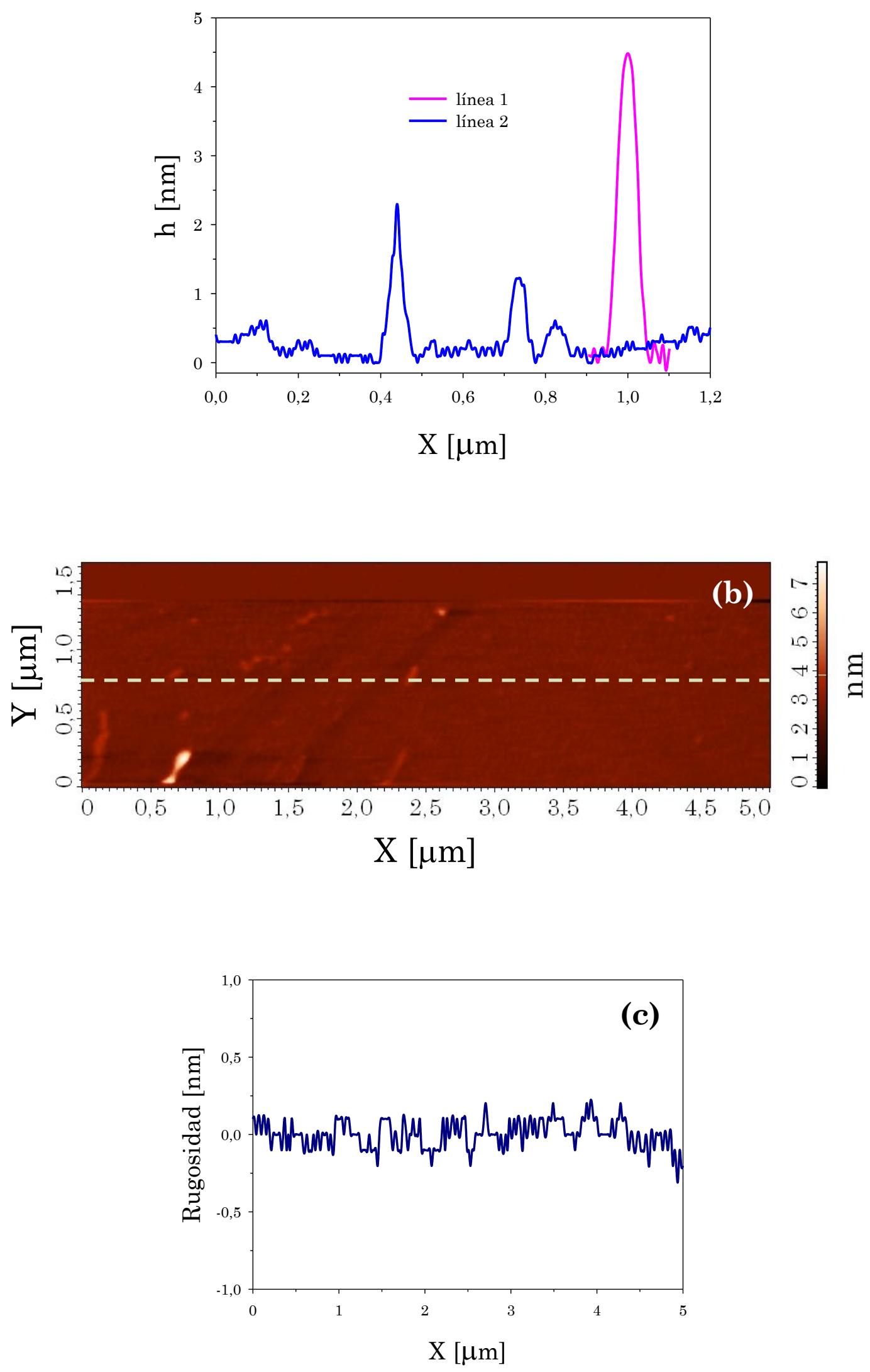


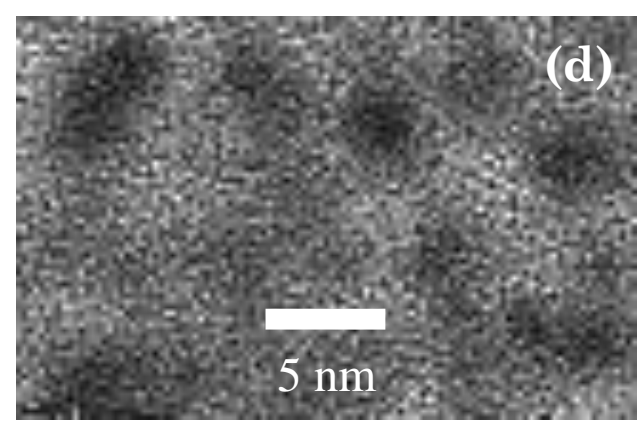

Figura 6.22: (a) En el panel superior se muestra la imagen AFM de la suspensión coloidal diluida obtenida por ablación láser con una energía de $500 \mu \mathrm{J}$ por pulso en agua. En el panel inferior se observa la altura (diámetro) de las partículas subnanométricas versus la posición X para las líneas 1 y 2 marcadas en la imagen del AFM. Observe el único pico en la línea 1 y dos picos de nanopartículas individuales en la línea 2. (b) Imagen AFM del sustrato de mica, (c) perfil de la medida de rugosidad del sustrato de mica que muestra un valor medio de 0,07 $\mathrm{nm}$ y (d) imagen HRTEM de nanopartículas de $\mathrm{Cu}-\mathrm{Cu}_{2} \mathrm{O}$. Los radios externos observados están en buen acuerdo con los valores obtenidos por la imagen de AFM y la espectroscopía de extinción [Santillán et al. 2012b].

Los resultados arrojados por espectroscopía de extinción óptica son consistentes con los obtenidos a partir de técnicas estándar tales como HRTEM y AFM. Además, la estadística de medición para espectroscopía de extinción óptica es muy grande, con valores típicos que van desde $10^{12}$ hasta $10^{14}$ nanopartículas, lo cual mejora la confiabilidad de las mediciones.

\section{- Plata}

Luego de haber realizado el estudio de la función dieléctrica de plata, teniendo en cuenta las contribuciones de electrones libres y ligados, se analiza el comportamiento teórico del coeficiente de extinción para diferentes estructuras y tamaños de partículas nanométricas y subnanoméricas.

En la Figura 6.23 (a) se muestra el coeficiente de extinción de una partícula core-shell de plata-óxido de plata $\left(\mathrm{Ag}_{2} \mathrm{O}\right)$ para un núcleo metálico 
de radio subnanométrico $a_{1}=0,7 \mathrm{~nm}$ cubierto por una delgada capa de $\mathrm{Ag}_{2} \mathrm{O}$ de $50 \% a_{1}$ de espesor, en medio acuoso, calculado con y sin corrección por tamaño de los electrones ligados. Los datos de la función dieléctrica experimental del óxido de plata fueron tomados de Qiu y coautores [Qiu et al. 2005] y se muestran en el recuadro de esta figura. Se observa que la posición del pico está alrededor de $485 \mathrm{~nm}$ para ambos espectros, pero las diferencias son más notorias para $\lambda \leq 520 \mathrm{~nm}$, donde la influencia de la parte imaginaria de los electrones ligados es más importante, mientras que para $\lambda \geq 520 \mathrm{~nm}$ los espectros son coincidentes.

En la Figura 6.23 (b) se representa el coeficiente de extinción de una partícula subnanométrica de plata de estructura simple $\left(a_{2}=a_{1}\right)$. Aquí, existe una discrepancia más evidente entre los espectros: la resonancia del plasmón de la partícula con corrección de los electrones ligados se encuentra en $375 \mathrm{~nm}$, mientras que para el cálculo sin corrección está ubicado en $385 \mathrm{~nm}$. Por otro lado, la resonancia del plasmón está desplazada hacia $485 \mathrm{~nm}$ (con shell de óxido) con respecto al pronunciado pico ubicado en $375 \mathrm{~nm}$ sin shell, cuando se realiza el cálculo con la corrección de los electrones ligados.

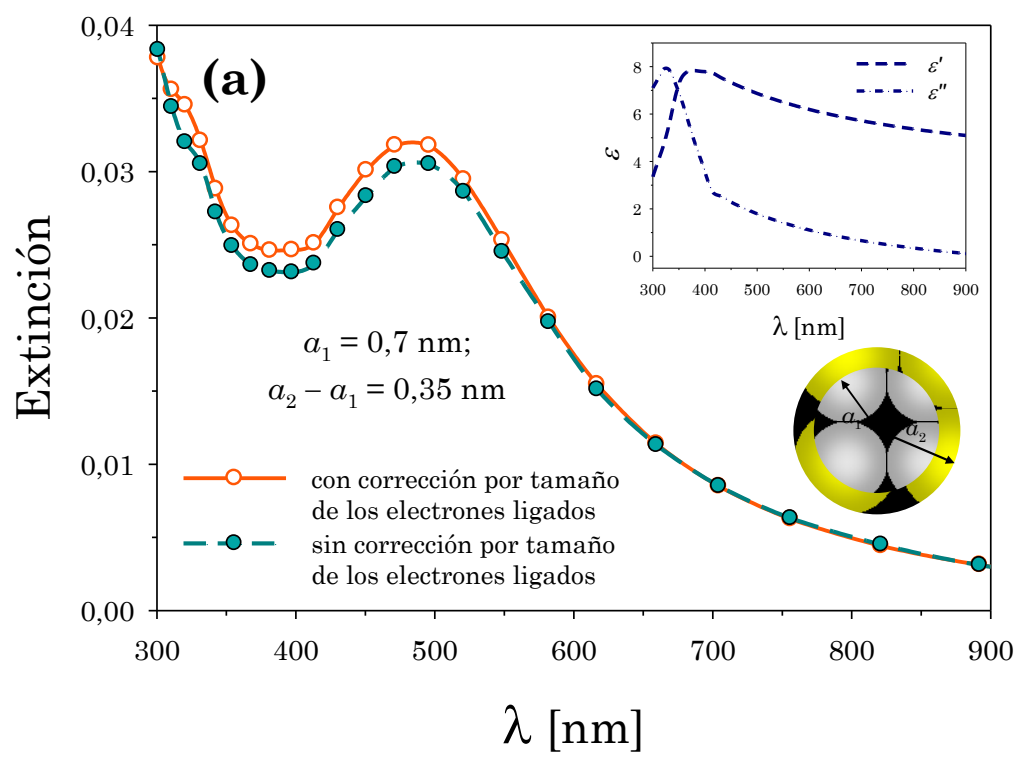




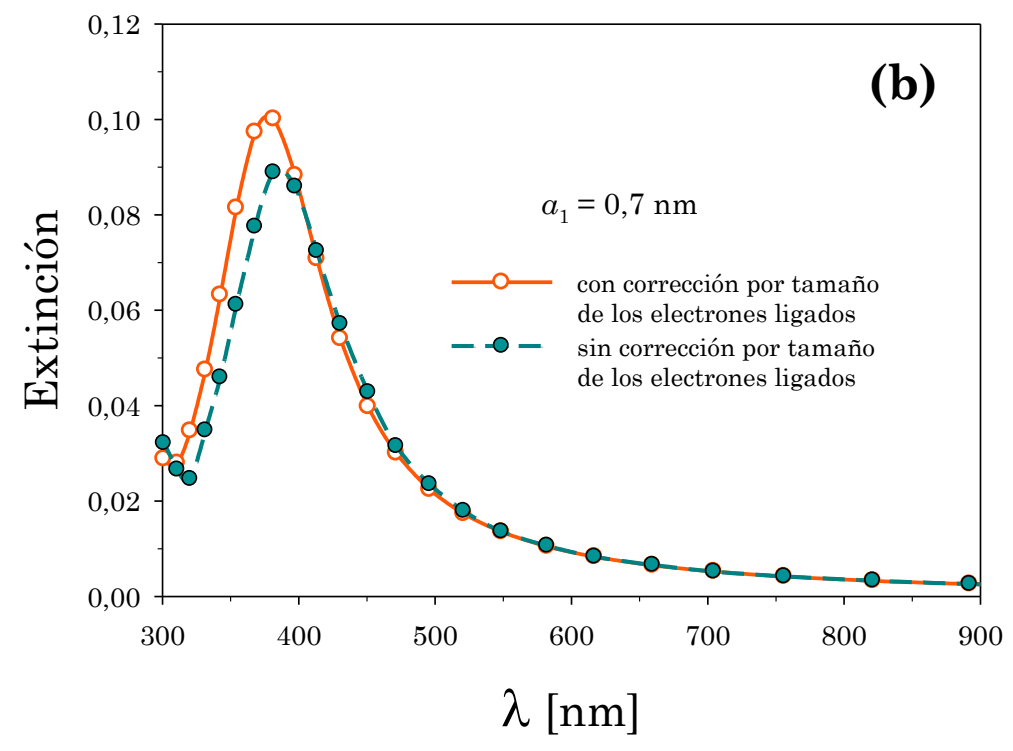

Figura 6.23: Coeficiente de extinción para una nanopartícula de Ag de estructura (a) core-shell y (b) simple, con y sin corrección por tamaño de los electrones ligados.

Para el ajuste completo del espectro de extinción experimental, especialmente en el rango de longitudes de onda largas, fue necesario considerar la inclusión de nanopartículas huecas de plata. Este tipo de nanopartículas huecas de estructura core-shell ha sido reportado por otros investigadores en distintos metales. Por ejemplo, Desarkar y coautores [Desarkar et al. 2013], fabricaron nanopartículas huecas de $\mathrm{Zn}-\mathrm{ZnO}$ por ablación láser en medios líquidos. Ben Moshe y Markovich [Ben Moshe and Markovich 2011], produjeron coloides de nanopartículas huecas de Ag con una rápida reducción química de nanopartículas de óxido de plata cubiertas con glutatión, mientras que Selvakannan y Sastry [Selvakannan and Sastry 2005], generaron nanopartículas huecas de oro cubiertas con platino a través de una reacción de transmetalización entre nanopartículas de Ag hidrofobizadas con cloroaurato hidrofobizado e iones de cloroplatinato hidrofobizado en cloroformo. 
Teniendo en cuenta estas consideraciones, fue posible analizar teóricamente el comportamiento de los espectros de extinción frente a la inclusión de nanopartículas huecas de Ag. La Figura 6.24 muestra el coeficiente de extinción para una nanopartícula de aire-Ag inmersa en agua, con un radio de $1 \mathrm{~nm}$ cubierto por una capa subnanométrica de $\mathrm{Ag}$ ( $\left.a_{2}-a_{1}=0,6 \mathrm{~nm}\right)$, calculado con y sin corrección por tamaño de los electrones ligados. Se observa que la curva calculada con la corrección por tamaño presenta mayor contraste entre el pico y el valle respecto a la curva calculada sin la corrección por tamaño de los electrones ligados.

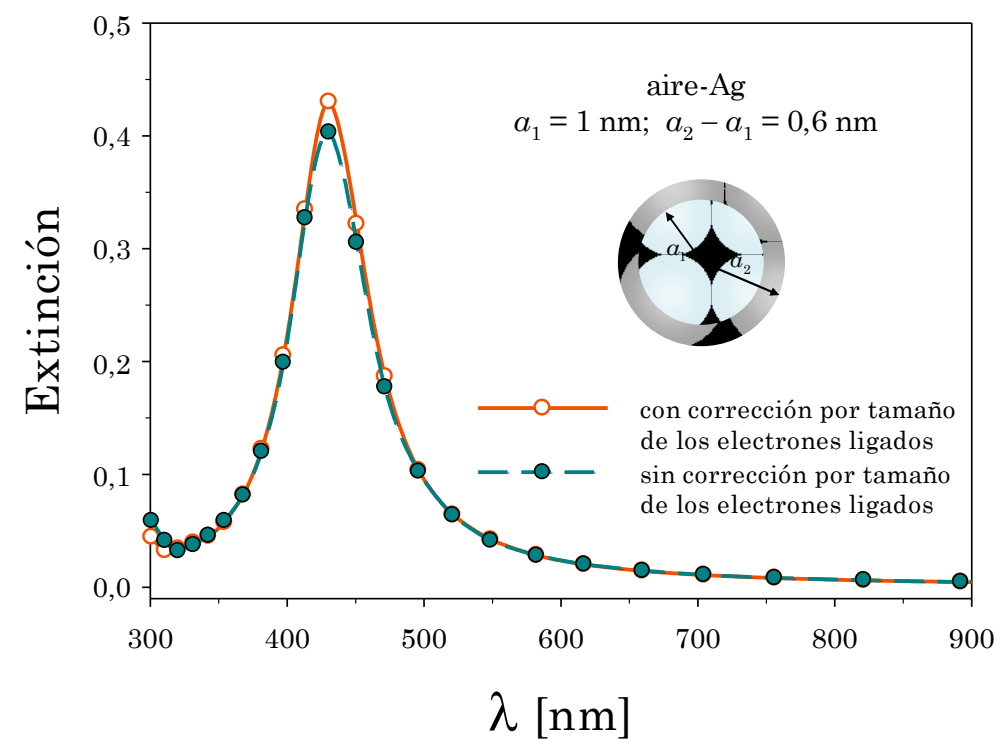

Figura 6.24: Espectros de extinción para una nanopartícula core-shell de aire-Ag con y sin corrección por tamaño de los electrones ligados.

Una vez analizado el comportamiento del coeficiente de extinción para diferentes estructuras, especies ( $\mathrm{Ag}, \mathrm{Ag}-\mathrm{Ag}_{2} \mathrm{O}$ o aire-Ag) y tamaños de partículas esféricas, se ajustaron los espectros de extinción experimentales correspondientes a las suspensiones coloidales de Ag obtenidas previamente por ablación láser. 
La Figura 6.25 muestra el mejor ajuste del espectro de extinción experimental normalizado correspondiente a una suspensión coloidal generada en agua por ablación láser de pulsos de femtosegundo con una energía por pulso de $500 \mu \mathrm{J}$.

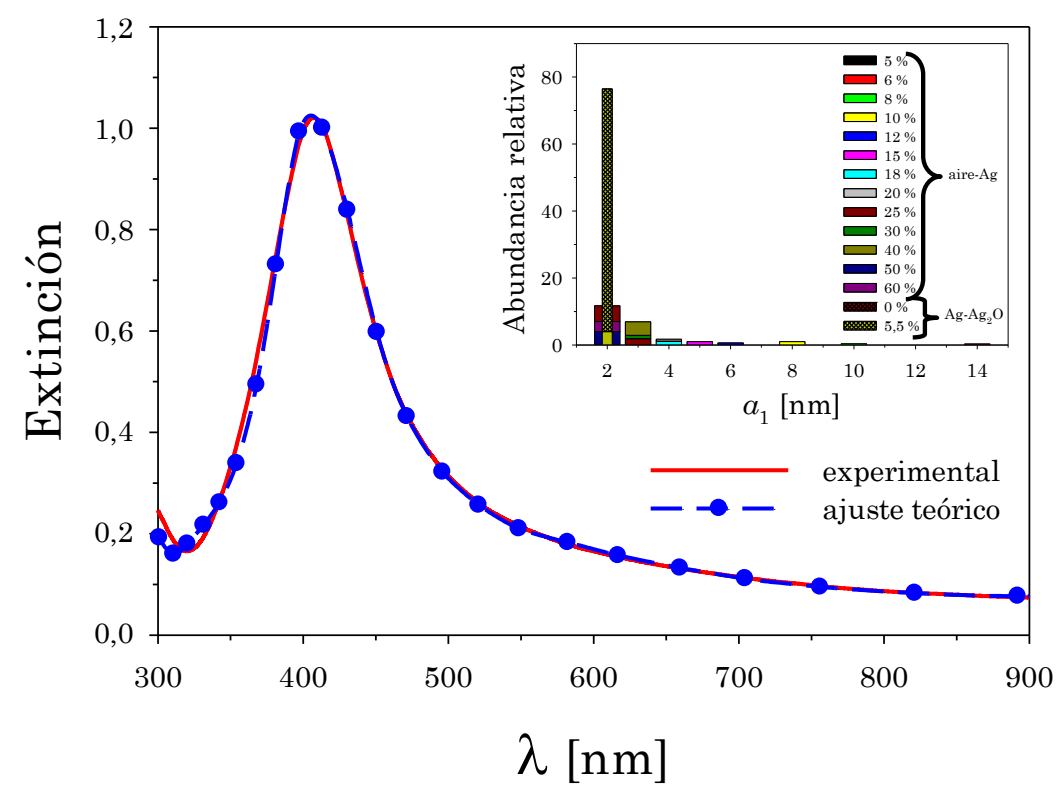

Figura 6.25: La línea roja corresponde al espectro de extinción experimental normalizado de nanopartículas de $\mathrm{Ag}-\mathrm{Ag}_{2} \mathrm{O}$ fabricadas en agua por ablación láser con una energía por pulso de $500 \mu \mathrm{J}$. La línea discontinua con círculos muestra el mejor ajuste calculado teóricamente con una contribución de nanopartículas de $\mathrm{Ag}, \mathrm{Ag}-\mathrm{Ag}_{2} \mathrm{O}$ y aire-Ag. El recuadro muestra la distribución de tamaño óptimo de radios de núcleos y espesores de recubrimiento que ajusta el espectro experimental [Santillán et al. 2013b].

El ajuste se obtuvo teniendo en cuenta dos tipos de estructuras: simple y core-shell. El primero consiste en nanopartículas de Ag, mientras que el segundo contiene dos especies de estructuras core-shell: nanopartículas de $\mathrm{Ag}-\mathrm{Ag}_{2} \mathrm{O}$ y aire-Ag. Considerando este tipo de estructuras, la distribución óptima que ajusta el espectro experimental tiene un radio del núcleo dominante de $2 \mathrm{~nm}$ y un espesor del recubrimiento de 5,5\% $a_{1}$ con una abundancia relativa de $72 \%$, de nanopartículas de $\mathrm{Ag}-\mathrm{Ag}_{2} \mathrm{O}$. Existe 
también una importante contribución de nanopartículas de aire-Ag con radios del núcleo de $2 \mathrm{~nm}$ y diferentes espesores del recubrimiento cuya abundancia relativa es de $12 \%$, y otro $12 \%$ de abundancia relativa de la misma especie de nanopartículas de diferentes radios y espesores del recubrimiento. Por último, existe una menor contribución de nanopartículas de Ag de estructura simple de $2 \mathrm{~nm}$ de radio con una abundancia relativa de 4\%. En el recuadro de esta figura se observa la abundancia relativa de los diferentes radios del núcleo con la distribución de espesores del recubrimiento para cada radio que corresponde al ajuste óptimo del espectro completo.

El ajuste teórico obtenido considerando la combinación de dos estructuras y especies de nanopartículas están en muy buen acuerdo con el resultado experimental obtenido para la suspensión coloidal.

Es interesante señalar también que ninguna combinación de partículas nanométricas y subnanométricas de estructura simple o coreshell podría ajustar por sí misma todo el espectro.

A modo de comparación de los resultados de tamaños obtenidos a través del método de espectroscopía de extinción óptica, se realizaron análisis de microscopía AFM y HRTEM de la misma muestra coloidal, como también se hizo para el cobre.

La Figura 6.26 (a) muestra, a modo de ejemplo, una imagen AFM de la muestra coloidal generada en agua.

Las partículas individuales fueron resueltas con claridad por imágenes de AFM de un área de escaneo de $7 \mu \mathrm{m}$ x $7 \mu \mathrm{m}$ utilizando muestras de baja concentración. Los perfiles de altura de las once líneas trazadas en la imagen AFM (Figura 6.26 (a)) se muestran en la Figura 6.26 (b). El escaneo de las líneas 1 a 11 incluye los siguientes tamaños de nanopartículas: $9,2 \mathrm{~nm}, 9,4 \mathrm{~nm}, 31,9 \mathrm{~nm}, 4,7 \mathrm{~nm}, 5 \mathrm{~nm}, 6 \mathrm{~nm}, 13,4 \mathrm{~nm}$, 
5,6 nm, 10,8 nm, 7,6 nm y 7,2 nm, respectivamente. Estas alturas están de acuerdo con la distribución de los radios externos determinados a partir del ajuste del espectro de extinción de la muestra. Para estos valores pequeños de tamaño, es importante tener en cuenta que la rugosidad de sustrato de mica (representado por el perfil de línea de base) en el que se depositó una gota de la muestra es más que un orden de magnitud menor que la partícula registrada más pequeña, lo que garantiza una muy buena señal-ruido en el perfil de las nanopartículas.

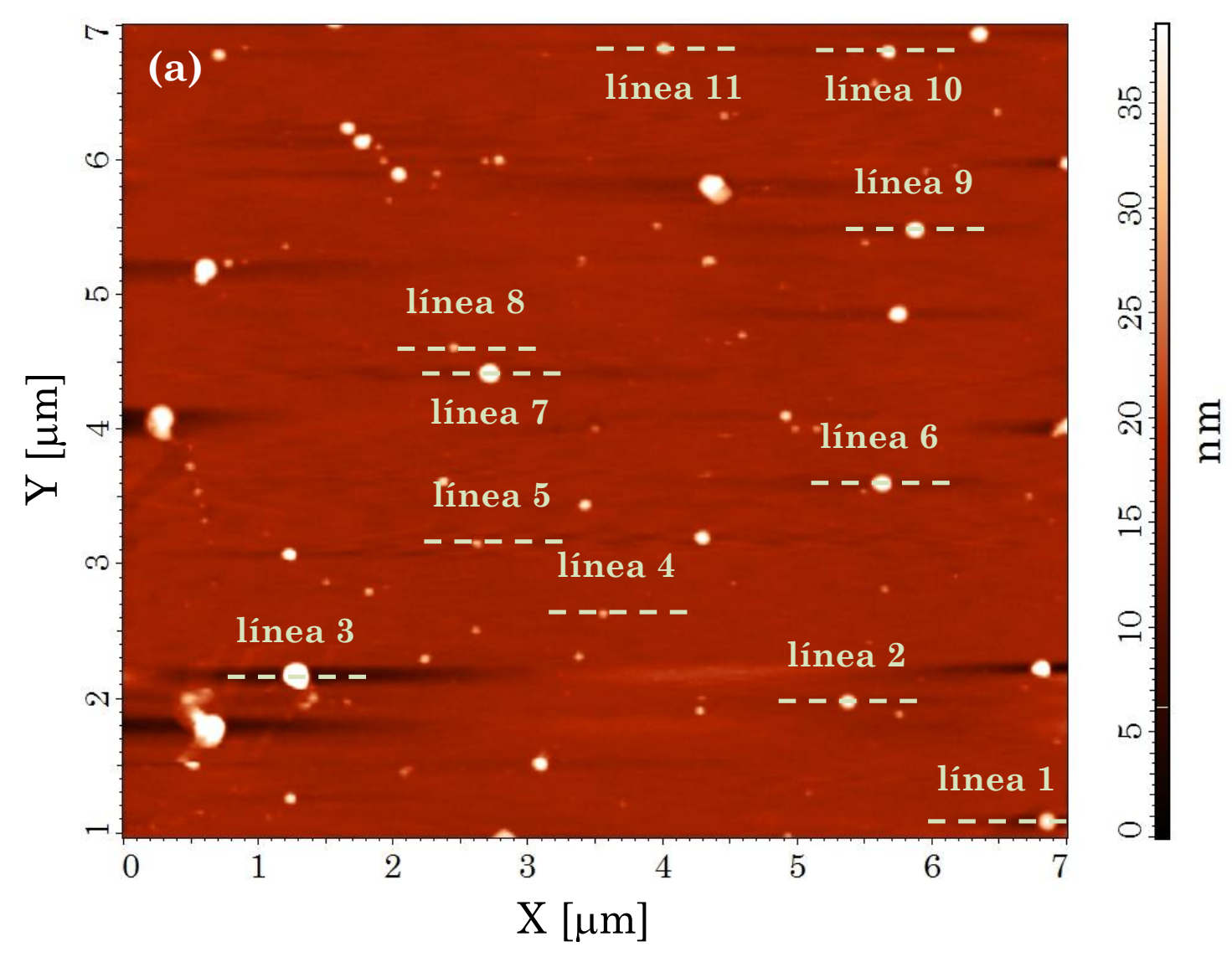



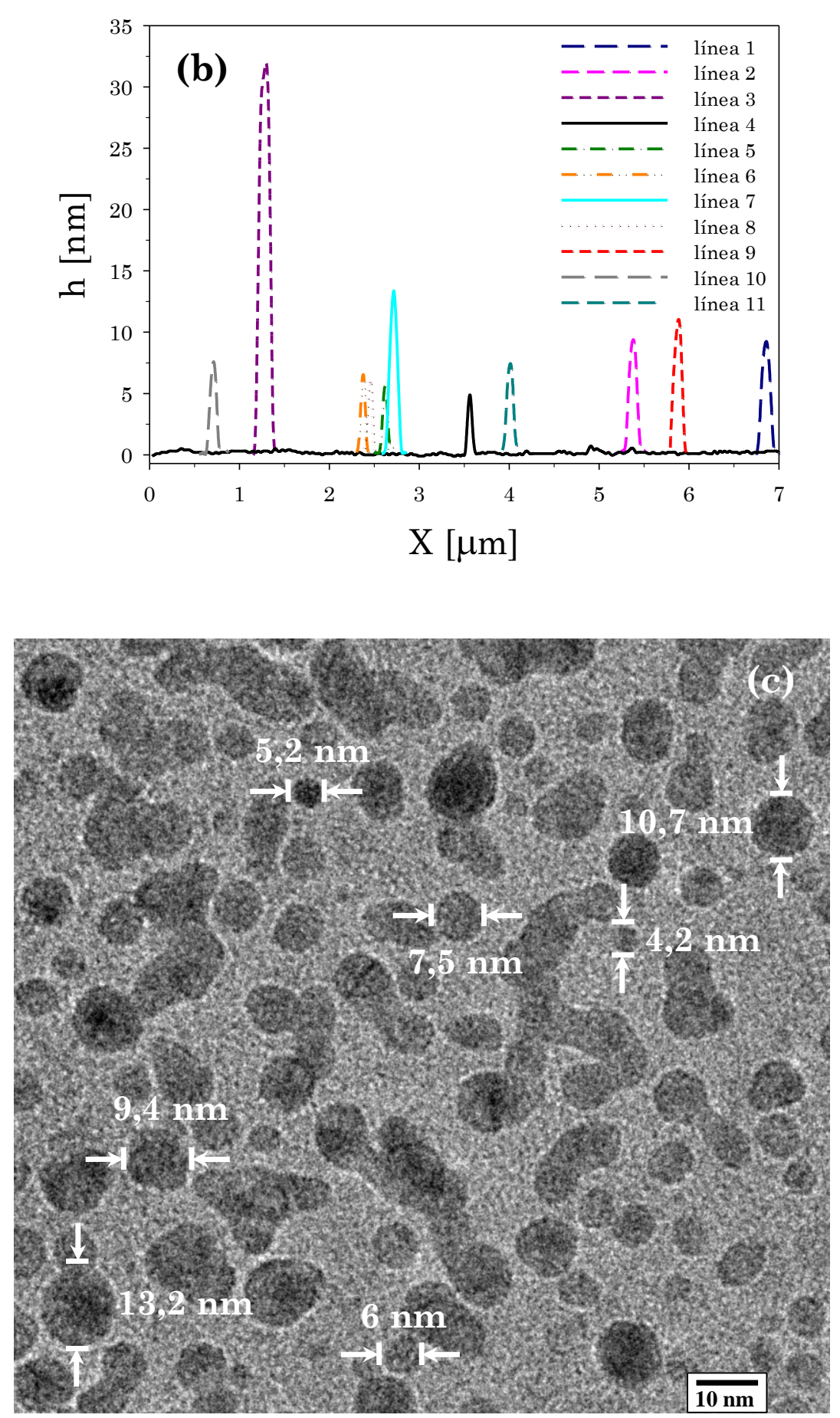

Figura 6.26: (a) Imagen AFM de la suspensión coloidal diluida de Ag generada en agua con una energía de $500 \mu \mathrm{J}$ por pulso. (b) Altura (diámetro) de las nanopartículas versus la posición X para las once líneas marcadas en la imagen del AFM. (c) Imagen HRTEM de la misma suspensión coloidal [Santillán et al. 2013b]. 
En la Figura 6.26 (c) se observa una imagen HRTEM de las nanopartículas core-shell coloidales de plata. La dilución de la muestra en agua fue suficiente para permitir la observación de partículas aisladas. Se puede ver que las nanopartículas son esféricas al igual que en la imagen de AFM de la Figura 6.26 (a), y en algunos casos, debido al método de preparación de la muestra, se puede notar efectos no deseados de coalescencia. Los tamaños típicos observados son consistentes con los resultados proporcionados por espectroscopía de extinción y microscopía AFM.

\subsection{Determinación de tamaños y estructuras de partículas nanométricas y subnanométricas}

Las propiedades ópticas tales como la extinción y la resonancia del plasmón son altamente dependientes del tamaño. En particular, resulta de interés estudiar las propiedades que presentan las partículas cuyos radios están por debajo de $10 \mathrm{~nm}$ debido a que manifiestan propiedades de funcionalización compatibles con marcadores para estudios biológicos. Estas propiedades también son dependientes del medio circundante y sobre todo del posible recubrimiento crecido alrededor de la partícula durante el proceso de fabricación (estructura core-shell). Estos procesos de fabricación pueden ser métodos de química húmeda o métodos físicos tales como la ablación láser de un blanco sólido en líquido o sputtering $\mathrm{RF}$, como se mencionó en el Capítulo 5.

En todos los casos, es necesario determinar el tamaño y la estructura de las nanopartículas fabricadas para poder determinar sus posibles aplicaciones. 
En tal sentido, esta Sección presenta el análisis de la estructura, la configuración (composición del núcleo y del recubrimiento) y el tamaño de las diferentes especies de partículas nanométricas y subnanométricas obtenidas por ablación láser de cobre en diferentes líquidos.

\subsubsection{Sobre la formación de óxido de cobre en nanopartículas}

Durante los últimos años, ha habido mucha investigación acerca de "nanocomposites" de Cu-óxido de Cu [Yin et al. 2005, Pike et al. 2006, Tilaki et al. 2007, Amikura et al. 2008, Pedersen et al. 2008, Ghodselahi and Vesaghi 2011, Kawasaki et al. 2011, Liu et al. 2011, Nath and Khare 2011] que cubren diferentes aspectos, como los métodos de fabricación, las propiedades ópticas y el análisis estructural. Desde un punto de vista general, se puede señalar que los mecanismos responsables de la formación de nanopartículas de $\mathrm{Cu}$-óxido de $\mathrm{Cu}$ no están del todo conocidos, y pueden variar debido a las diferentes condiciones en las que fueron fabricadas. Yin y coautores [Yin et al. 2005] desarrollaron una técnica de formación de coloides de $\mathrm{Cu}$ por descomposición térmica de alta temperatura de acetato de cobre (I), que fue oxidado gradualmente para formar partículas estables de $\mathrm{Cu}_{2} \mathrm{O}$ de $6 \mathrm{~nm}$ aproximadamente. Sin embargo, algunos de sus resultados de absorción óptica muestran una resonancia alrededor de $610 \mathrm{~nm}$, que fue asignado tentativamente por los autores a un posible pico del plasmón de nanopartículas de $\mathrm{Cu}-\mathrm{Cu}_{2} \mathrm{O}$. Pike y colaboradores [Pike et al. 2006] utilizaron difracción de rayos X (XRD, X-Ray Diffraction) con resolución temporal para estudiar un experimento de reducción aplicando una rampa de temperatura donde muestran que, mientras se reduce $\mathrm{CuO}$ directamente a $\mathrm{Cu}$ en tamaño macroscópico, en la nanoescala esta reducción se realiza en dos pasos, primero se produce la reducción al $\mathrm{Cu}_{2} \mathrm{O}$ estable y luego al $\mathrm{Cu}$ a 
medida que aumenta la temperatura. A partir de sus experimentos sugieren que debería haber un tamaño crítico de partícula por debajo del cual se produce esta reducción secuencial. Pedersen y coautores [Pedersen et al. 2008] han generado nanopartículas de $\mathrm{Cu}$ utilizando sputtering con magnetrón $\mathrm{DC}$ de un blanco de $\mathrm{Cu}$ de alta pureza sobre un portaobjeto de vidrio en una atmósfera libre de oxígeno. Después de una exposición al aire a $160^{\circ} \mathrm{C}$, obtuvieron nanopartículas de $\mathrm{Cu}$ de $3 \mathrm{~nm}$ de diámetro, y los espectros de absorción tomados para diferentes tiempos mostraron una reducción del pico del plasmón de $\mathrm{Cu}$ y un refuerzo de las bandas del UV por debajo de $350 \mathrm{~nm}$, compatible con la formación de nanopartículas de $\mathrm{Cu}-\mathrm{Cu}_{2} \mathrm{O}$. Además, la oxidación de pequeñas nanopartículas de $\mathrm{Cu}$ produjo diferentes tipos de óxido dependiendo del rango de temperaturas en el que se genera el proceso. Una técnica de fabricación de nanopartículas de $\mathrm{Cu}$ utilizada por muchos autores es la ablación láser pulsada de un blanco sólido en líquidos [Tilaki et al. 2007, Amikura et al. 2008, Nath and Khare 2011, Liu et al. 2011], descripta en el Capítulo 5. En estos casos, se utilizó la longitud de onda fundamental o la segunda armónica de un láser de Nd:YAG de $10 \mathrm{~ns}$ de duración por pulso. Tilaki y colaboradores [Tilaki et al. 2007] generaron con este mismo método nanopartículas de $\mathrm{Cu}$ en agua y acetona con tamaños alrededor de $30 \mathrm{~nm}$ y $3 \mathrm{~nm}$, respectivamente. Los espectros de extinción óptica obtenidos a diferentes tiempos después de la fabricación muestran una resonancia del plasmón de $\mathrm{Cu}$ alrededor de $600 \mathrm{~nm}$, aunque es algo diferente de la resonancia típica del $\mathrm{Cu}$ de $590 \mathrm{~nm}$ obtenida a partir de los cálculos de Mie. Para explicar esta diferencia, los autores proponen, entre otras razones, la oxidación del coloide de cobre debido a la reacción con el oxígeno disuelto en el agua, aunque no existe ningún intento de ajuste de los espectros experimentales. Amikura y coautores [Amikura et al. 2008] y Nath y Khare [Nath and Khare 2011] utilizaron la segunda armónica de un láser de Nd:YAG de $10 \mathrm{~ns}$ de duración por pulso para la ablación de un blanco de cobre en agua. Los primeros 
producen partículas de óxido cuproso $\left(\mathrm{Cu}_{2} \mathrm{O}\right)$ de varios tamaños y morfologías, aunque en todos los casos los espectros UV-visible muestran invariablemente un pico en $650 \mathrm{~nm}$, que los autores asignaron tentativamente al plasmón del $\mathrm{Cu}$ y finalmente a nanopartículas esféricas de $\mathrm{Cu}$-óxido de $\mathrm{Cu}$. Los últimos, investigaron la influencia de las condiciones de enfoque (fluencia) en el tamaño y las características del óxido de cobre de los coloides resultantes. Basados en los resultados de espectroscopía de extinción óptica, como en espectroscopía Raman de los coloides, llegaron a concluir que se generan nanopartículas de $\mathrm{Cu}$-óxido de $\mathrm{Cu}$, tanto con óxido cúprico $(\mathrm{CuO})$ como con óxido cuproso $\left(\mathrm{Cu}_{2} \mathrm{O}\right)$, logrando tamaños menores a $200 \mathrm{~nm}$ en condiciones de máximo enfoque e inferiores a $10 \mathrm{~nm}$ en condiciones de desenfoque, respectivamente.

Recientemente, Liu y coautores [Liu et al. 2011] utilizaron la longitud de onda fundamental de un láser de Nd:YAG para producir nanopartículas de $\mathrm{Cu}_{2} \mathrm{O}$ de $30 \mathrm{~nm}$ de diámetro por ablación láser de una placa de cobre electrolítico inmerso en PVP. El análisis de los patrones de XRD y de difracción de electrones mostró que las partículas de $\mathrm{Cu}$ secadas a temperatura ambiente se oxidan completamente a $\mathrm{Cu}_{2} \mathrm{O}$.

Los mecanismos que subyacen a la formación de nanopartículas de cobre por ablación láser pulsada de un blanco sólido de cobre en líquidos son muy complejos. Los procesos de oxidación de nanopartículas de $\mathrm{Cu}$ son complicados debido a que el cobre tiene dos estados de oxidación $\left(\mathrm{Cu}^{+1}\right.$ y $\mathrm{Cu}^{+2}$ ) que forman óxidos estables con diferentes barreras en la energía de activación. Las condiciones de extrema presión y temperatura en el volumen focal durante el proceso de ablación láser y su rápida variación en el tiempo hacen que la explicación acerca de la formación del óxido de cobre sea una tarea difícil. Tilaki y coautores [Tilaki et al. 2007] mencionan el rol de la interacción del plasma generado en la pluma-nanopartícula en el mecanismo de crecimiento de nanopartículas por procesos de nucleación, así como la 
oxidación de coloidales de $\mathrm{Cu}$ debido a la reacción con el oxígeno disuelto en el agua. Amikura y colaboradores [Amikura et al. 2008] apoyan la idea compartida también por Pedersen y coautores [Pedersen et al. 2008]. Kabashin y coautores [Kabashin and Meunier 2006] hacen notar que el mecanismo de formación incluye diversas reacciones en un ambiente plasma-líquido de alta temperatura y alta presión fuera del equilibrio, que culminan con la formación de nanopartículas en las burbujas de cavitación generadas en el breakdown láser. Los clusters de $\mathrm{Cu}$ se forman después de la expansión adiabática (y enfriamiento) luego de que el plasma generado en la pluma reacciona con el solvente (radicales oxidrilos o átomos de oxígeno) para producir óxidos de cobre. Nath y Khare [Nath and Khare 2011] describen la formación de nanopartículas de óxido de $\mathrm{Cu}$ en un régimen de nanosegundos de ablación láser pulsada de alta potencia para diferentes condiciones de enfoque. Similarmente al trabajo de Kabashin y coautores, argumentan que la onda de choque que resulta del breakdown del láser en el medio genera condiciones de alta presión y temperatura en la interfase líquido-plasma, que se convierte en una zona de reacción química activa mejorando la unión de las especies de cobre con las moléculas de agua ionizada para formar nanopartículas de óxido de $\mathrm{Cu}$. Las condiciones de extrema presión y temperatura encontradas en la interfase blanco sólidolíquido dominan el tamaño y la fase estructural de las nanopartículas sintetizadas. Los resultados de los espectros Raman indican que para nanosegundos en el régimen de baja fluencia es más probable que se generen nanopartículas de $\mathrm{Cu}_{2} \mathrm{O}$ en lugar de nanopartículas de $\mathrm{CuO}$. Además, a partir de los espectros de absorción UV-visible concluyen que también se forman estructuras core-shell $\mathrm{Cu}-\mathrm{Cu}_{2} \mathrm{O}$ durante el proceso de ablación. 


\subsubsection{Influencia del shell de $\mathrm{Cu}_{2} \mathrm{O}$ en los espectros de extinción}

Considerando los trabajos citados anteriormente, fue apropiado analizar las características de los espectros de extinción óptica de pequeñas nanopartículas esféricas de estructura core-shell de $\mathrm{Cu}-\mathrm{Cu}_{2} \mathrm{O}$.

A partir de las ecuaciones (6.1) y (2.34), fue posible calcular el coeficiente de extinción para partículas nanométricas y subnanométricas de cobre con y sin recubrimiento de $\mathrm{Cu}_{2} \mathrm{O}$. En la Figura 6.27 (a) se comparan los espectros de extinción normalizados de dos tipos de estructuras: esfera simple y esfera core-shell, donde el radio del núcleo de ambas partículas es $0,7 \mathrm{~nm}$ y el espesor del recubrimiento $\left(\mathrm{Cu}_{2} \mathrm{O}\right)$ de la partícula core-shell es $0,35 \mathrm{~nm}$ (50\% del radio del núcleo). Se puede observar que existe un desplazamiento hacia el rojo considerable de la posición del pico (alrededor de $70 \mathrm{~nm}$ en este caso) y un aumento de la pendiente para longitudes de onda por debajo de $600 \mathrm{~nm}$ en el espectro de extinción cuando se considera el recubrimiento de óxido.

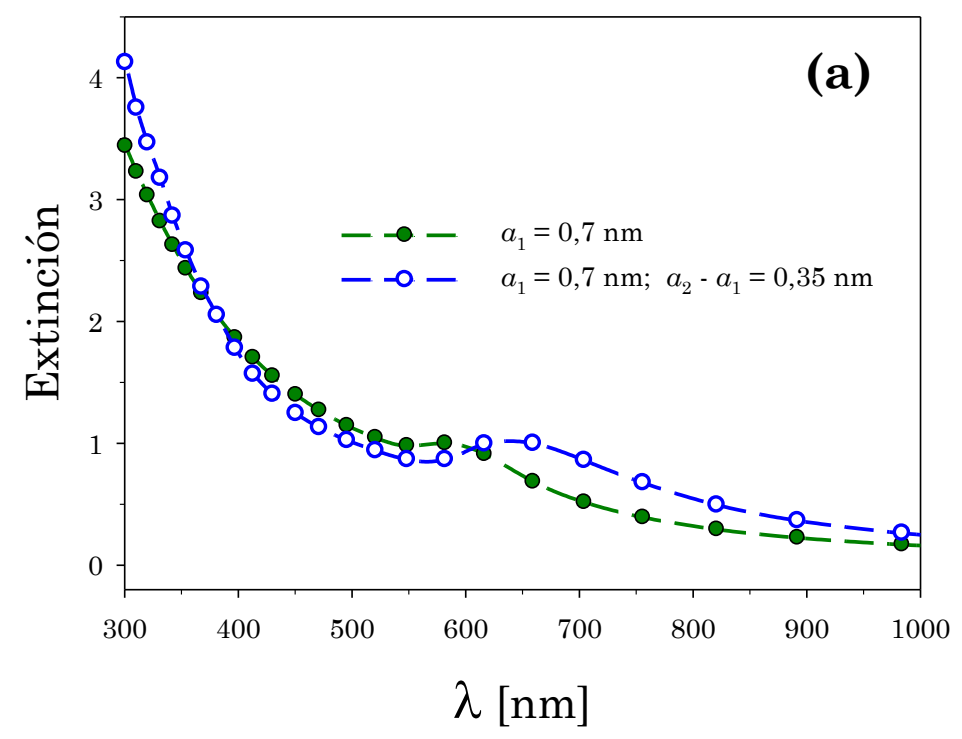




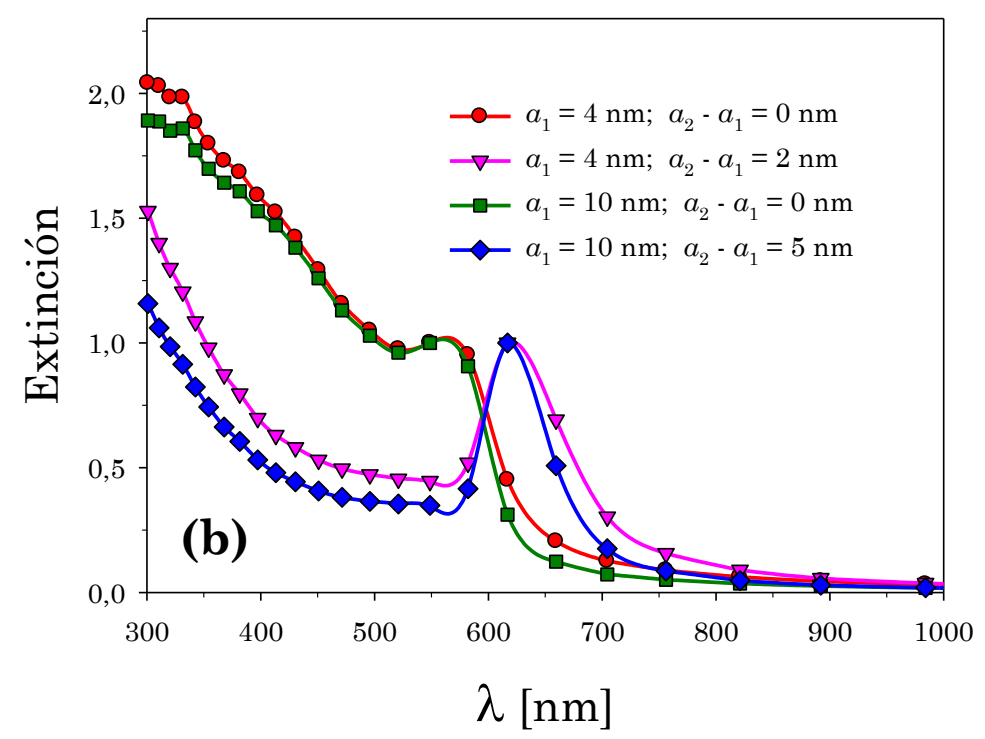

Figura 6.27: Espectros de extinción óptica calculados, ambos normalizados, para nanopartículas de $\mathrm{Cu}$ de estructura simple comparados con nanopartículas de estructura core-shell con el mismo radio del núcleo y $50 \%$ de espesor de $\mathrm{Cu}_{2} \mathrm{O}$ : (a) subnanométrica y (b) nanométrica. El shell produce un desplazamiento medible del pico del plasmón.

La Figura 6.27 (b) muestra un comportamiento similar para nanopartículas de cobre de $4 \mathrm{~nm}$ y $10 \mathrm{~nm}$ de radio, con un espesor de $\mathrm{Cu}_{2} \mathrm{O}$ de $0 \%$ (núcleo desnudo) y $50 \% a_{1}$ (núcleo-recubrimiento). En este caso el shell también produce un desplazamiento notable hacia el rojo junto con una definición más aguda del pico. Además, la cola de longitudes de onda cortas de nanopartículas core-shell tiene una forma muy diferente en comparación con las nanopartículas de núcleo desnudo.

\subsubsection{Ajuste de los espectros de extinción de las suspensiones coloidales}

Habiendo realizado un análisis del comportamiento de la posición del pico del plasmón y la forma del espectro completo en función del tamaño del núcleo y espesor del shell, se pudieron caracterizar los espectros de extinción 
de las suspensiones coloidales fabricadas por ablación de un blanco sólido de $\mathrm{Cu}$ en agua y acetona.

Los espectros experimentales de las suspensiones coloidales de cobre fabricadas mediante ablación láser de pulsos ultracortos en diferentes medios líquidos y diferentes energías de pulso se muestran en las siguientes figuras. Todos los espectros experimentales fueron tomados 5 minutos después de la fabricación y se normalizaron a la unidad en la resonancia del pico del plasmón a efectos de comparación. Las características generales de los espectros (posición del pico y las colas a longitudes de onda cortas y largas) son muy similares a los obtenidos por Tilaki y coautores [Tilaki et al. 2007], a pesar de que los autores nunca intentaron un ajuste completo del espectro.

En las Figuras 6.28 (a) y (b) se representan los espectros de extinción experimental obtenidos a diferentes energías de pulso de fs entre $500 \mu \mathrm{J}$ y $50 \mu \mathrm{J}$ en acetona y agua, respectivamente.

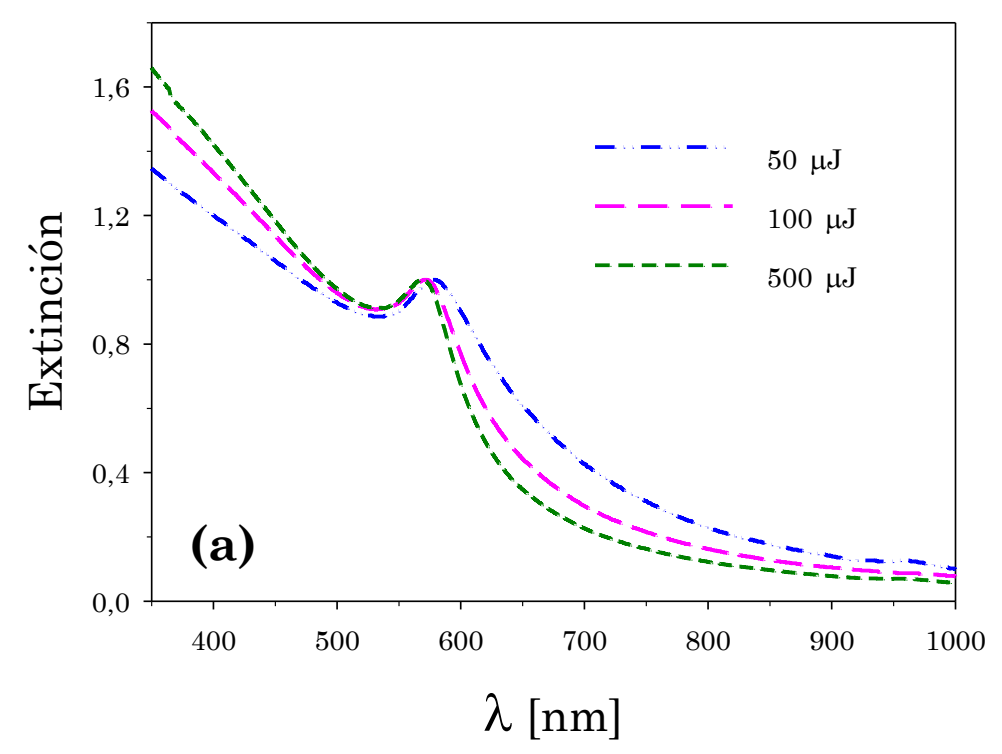




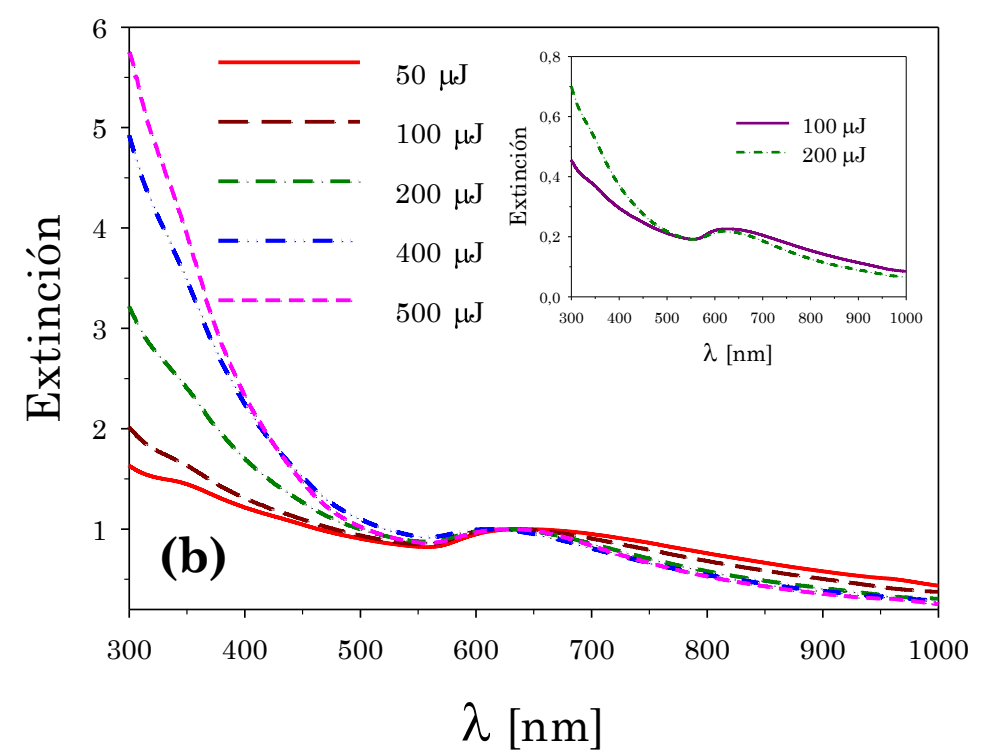

Figura 6.28: Espectros de extinción experimentales de suspensiones coloidales de cobre fabricadas por ablación láser de pulsos de fs a diferentes energías y diferentes medios: (a) en acetona y (b) en agua. El recuadro muestra un detalle de los espectros correspondientes a dos energías típicas.

Es interesante notar que estos espectros normalizados tienen formas diferentes a medida que la energía pasa de valores altos a valores más bajos y la longitud de onda del pico del plasmón está desplazada hacia el rojo para energías bajas (Figura 6.28 (a)). La diferencia en la forma de los espectros es más evidente para el caso del agua (Figura 6.28 (b)), particularmente en la cola de longitudes de onda cortas de 300 a $500 \mathrm{~nm}$. El recuadro muestra por separado los espectros correspondientes a $100 \mu \mathrm{J}$ y $200 \mu \mathrm{J}$, donde se puede observar no sólo la diferencia en la pendiente de la cola de longitudes de onda cortas, sino también un leve desplazamiento hacia el rojo del pico del plasmón. Más adelante se discutirá el comportamiento de las curvas del recuadro.

La Figura 6.29 muestra las curvas experimentales (línea sólida roja) y los ajustes teóricos normalizados (rombo y línea discontinua verde) que corresponden a las suspensiones coloidales de cobre en acetona fabricadas 
con (a) $500 \mu \mathrm{J}$, (b) $100 \mu \mathrm{J} \mathrm{y} \mathrm{(c)} 50 \mu \mathrm{J}$ de energía por pulso, respectivamente. El ajuste de las curvas experimentales se realizó usando las expresiones (6.1) y (2.34), considerando las diferentes estructuras esféricas con dos tipos de configuraciones de núcleo y recubrimiento y una distribución de tamaños y espesores del recubrimiento. El recuadro en cada panel muestra la abundancia relativa de los diferentes radios del núcleo con la distribución de espesores del recubrimiento para cada radio que corresponde al ajuste óptimo del espectro completo. Aquí se debe señalar que los histogramas de la distribución que corresponde al ajuste de los espectros experimentales son el resultado de un proceso iterativo que cambia secuencialmente diferentes parámetros, tales como el radio del núcleo, el espesor del recubrimiento y la configuración core-shell. Cada parámetro modifica una característica independiente de los espectros (pico y ancho del plasmón y la pendiente a longitudes de onda cortas y largas), de manera que el conjunto óptimo es único en el sentido de que no existe otro conjunto de parámetros que ajusten correctamente al espectro completo.

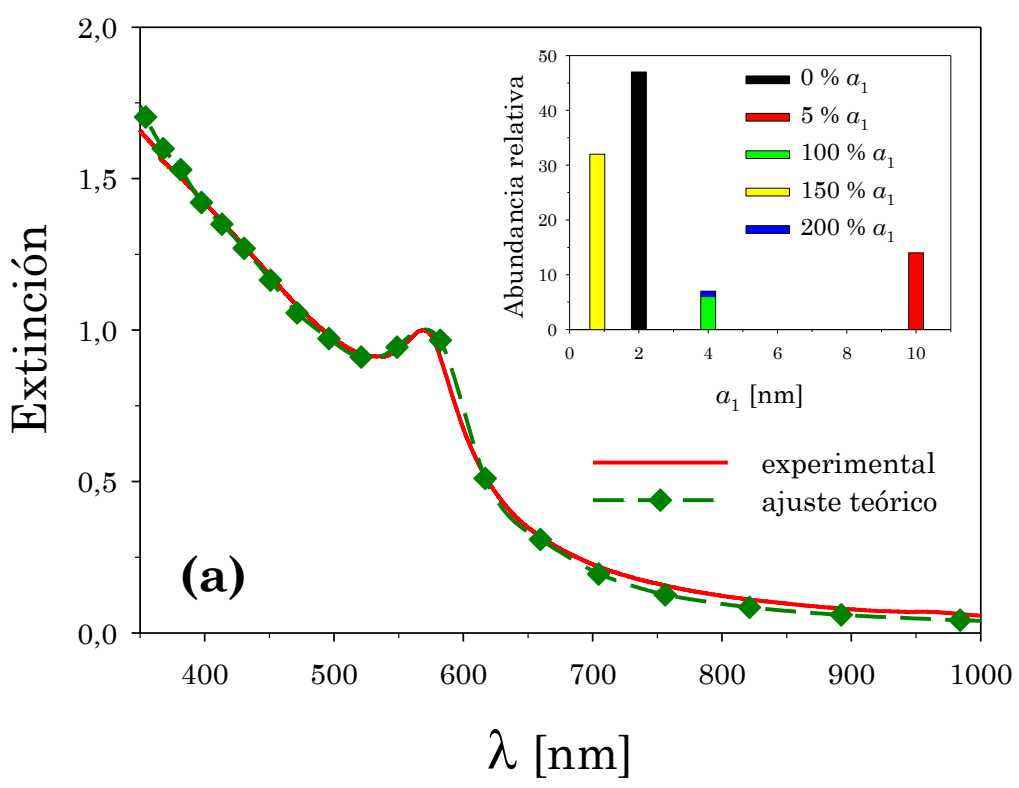



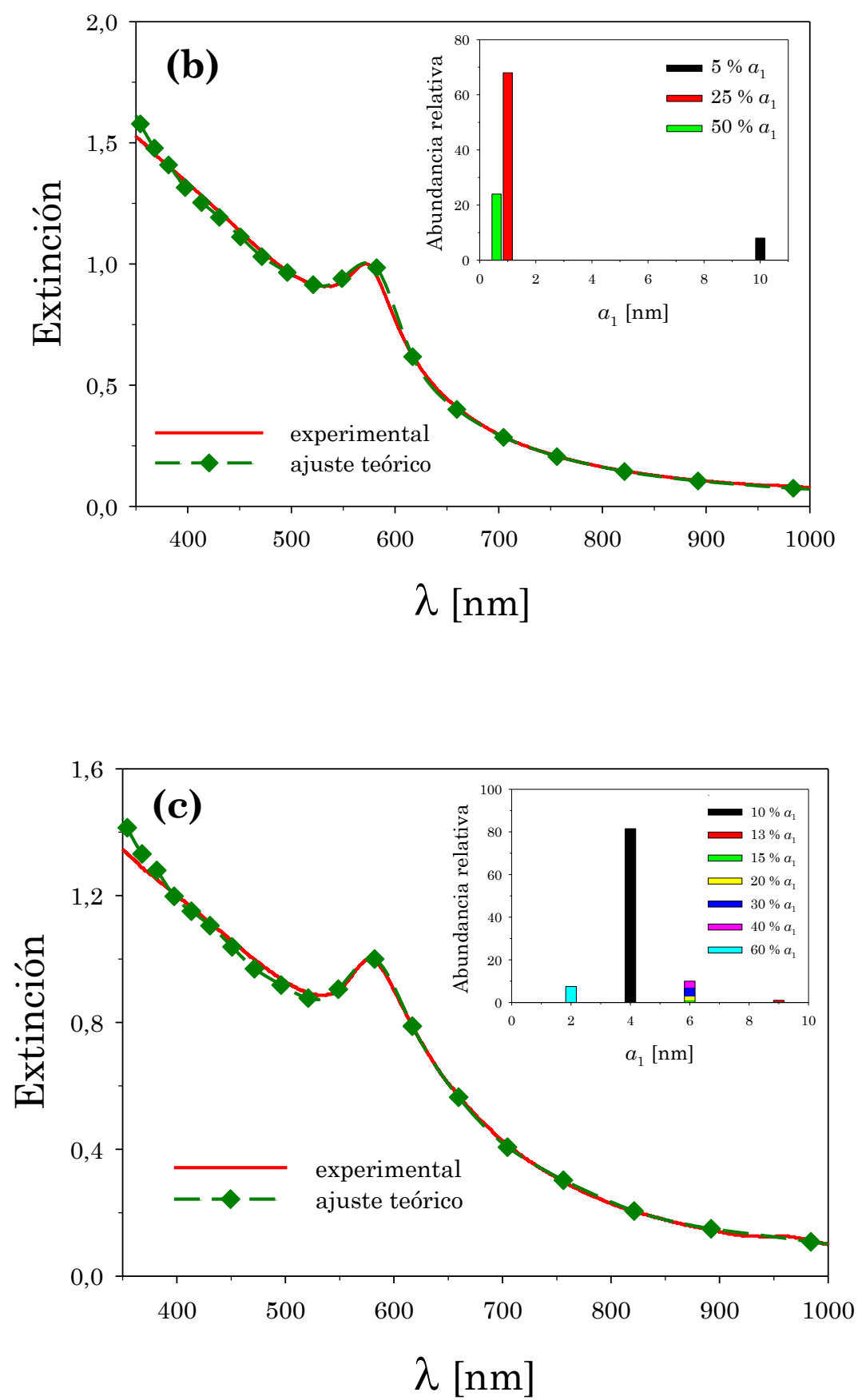

Figura 6.29: Espectros experimentales y ajustes teóricos normalizados de suspensiones coloidales en acetona: energía por pulso (a) $\mathrm{E}=500 \mu \mathrm{J}$, (b) $\mathrm{E}=100 \mu \mathrm{J}$ y (c) $\mathrm{E}=50 \mu \mathrm{J}$. En cada panel, el recuadro muestra la distribución de tamaño óptima de los radios del núcleo y espesores del shell que se ajusta al espectro experimental. El ajuste en el panel (a) se basa en una combinación de estructuras simples $(\mathrm{Cu})$ y core-shell $\left(\mathrm{Cu}-\mathrm{Cu}_{2} \mathrm{O}\right)$. En el panel (b) el ajuste se centra solamente en una estructura de $\mathrm{Cu}-\mathrm{Cu}_{2} \mathrm{O}$, mientras que el ajuste en el panel (c) se basa en una combinación de configuraciones de $\mathrm{Cu}-\mathrm{Cu}_{2} \mathrm{O}$ y $\mathrm{Cu}_{2} \mathrm{O}-\mathrm{Cu}$. 
Para la Figura 6.29 (a) la distribución de tamaño óptimo tiene un radio dominante de $\mathrm{Cu}$ de $2 \mathrm{~nm}$ e incluye una abundancia de $7 \%$ y $14 \%$ para radios de 4 y $10 \mathrm{~nm}$, respectivamente, de nanopartículas de $\mathrm{Cu}-\mathrm{Cu}_{2} \mathrm{O}$. Existe también una importante contribución de partículas de radio subnanométrico de $0,8 \mathrm{~nm}$ de la misma configuración cuya abundancia es de $32 \%$. Es importante destacar el hecho de que los radios del núcleo y espesores del recubrimiento que aparecen en el histograma representado en el recuadro indican una distribución bimodal compuesta por pequeños radios en el rango de 1 a $4 \mathrm{~nm}$ y una más grande centrada en $10 \mathrm{~nm}$.

El ajuste del espectro en la Figura 6.29 (b) muestra también una distribución bimodal, con nanopartículas de $\mathrm{Cu}-\mathrm{Cu}_{2} \mathrm{O}$ de radios nanométricos y subnanométricos. El primero tiene una abundancia de $68 \%$ de nanopartículas de $1 \mathrm{~nm}$ de radio del núcleo con 0,25 $\mathrm{nm}$ de espesor de $\mathrm{Cu}_{2} \mathrm{O}$ y $24 \%$ de abundancia de partículas subnanométricas de $0,6 \mathrm{~nm}$ de radio y $0,3 \mathrm{~nm}$ de espesor de $\mathrm{Cu}_{2} \mathrm{O}$, mientras que el segundo tiene un pequeño aporte de porcentaje de partículas de $10 \mathrm{~nm}$ de radio y $0,5 \mathrm{~nm}$ de espesor de $\mathrm{Cu}_{2} \mathrm{O}$. A pesar de que esta última abundancia es pequeña en comparación con las anteriores, es importante incluirla ya que las partículas grandes tienen mayor espectro de extinción que las pequeñas y se ajusta mejor a la cola de longitudes de onda grandes del espectro experimental. El espectro experimental de $50 \mu \mathrm{J}$ de energía se muestra en la Figura 6.29 (c) y merece especial atención. El ajuste teórico correspondiente sólo puede obtenerse considerando dos configuraciones de tipo core-shell: un $81 \%$ de nanopartículas de $\mathrm{Cu}-\mathrm{Cu}_{2} \mathrm{O}$ con un radio del núcleo de $4 \mathrm{~nm}$ y el $19 \%$ restante corresponde a nanopartículas de $\mathrm{Cu}_{2} \mathrm{O}-\mathrm{Cu}$ con un radio del núcleo diferente. La inclusión de esta última configuración permitió obtener un ajuste adecuado de la cola de longitudes de onda largas. Es preciso señalar que era imposible ajustar todo el espectro con un solo tipo de configuración core-shell. Esta característica se encontrará de nuevo al describir los espectros de extinción en agua. 
Para el caso de las suspensiones coloidales fabricadas en agua, se tendrá en cuenta el espectro experimental ajustado en la Figura 6.18 que corresponde a una energía de $500 \mu \mathrm{J}$, para la discusión de resultados. La Figura 6.30 (a) muestra la extinción espectral experimental (línea sólida roja) y el ajuste teórico (círculos y línea discontinua negra) que corresponde a una suspensión coloidal de nanopartículas en agua fabricadas con $100 \mu \mathrm{J}$ de energía por pulso. En la Figura 6.30 (b) se puede ver que el espectro experimental muestra tres picos característicos en el UV en el rango de 300 a $360 \mathrm{~nm}$, que corresponden a las transiciones de Brillouin del $\mathrm{Cu}_{2} \mathrm{O}$, por un lado, mientras que por el otro se observa un pico ensanchado alrededor de $650 \mathrm{~nm}$ correspondiente a la presencia del plasmón de una nanoestructura de cobre, de acuerdo con los espectros analizados por los autores Nath y Khare [Nath and Khare 2011].

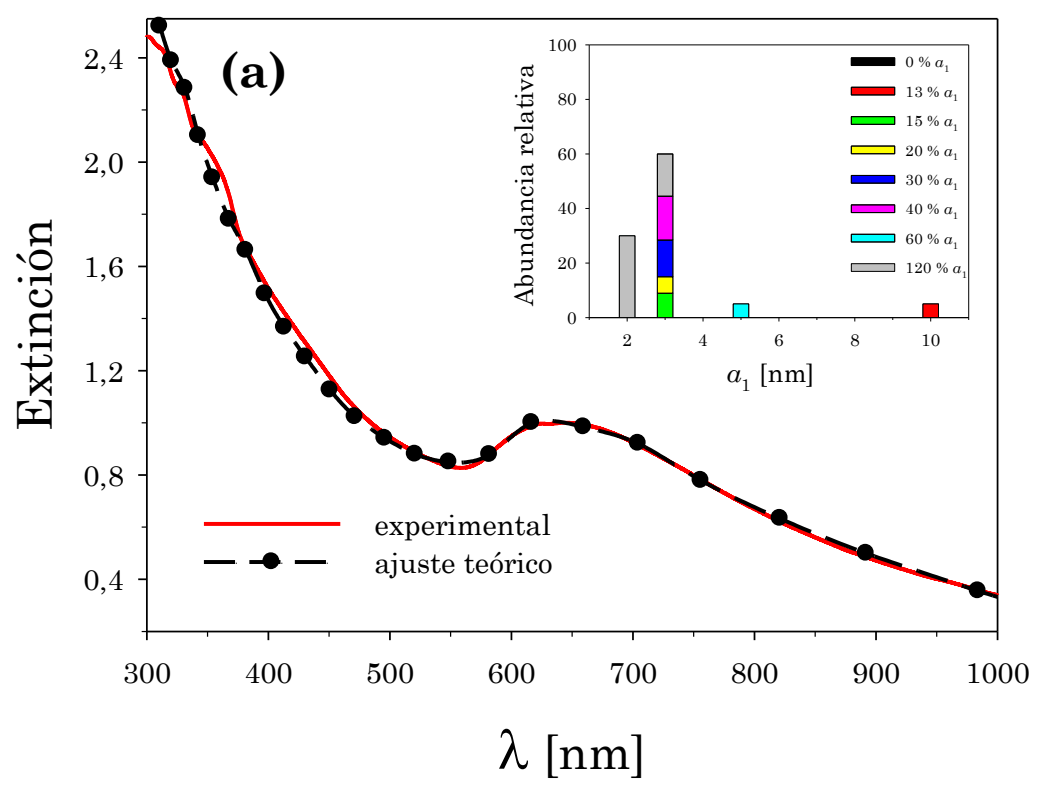




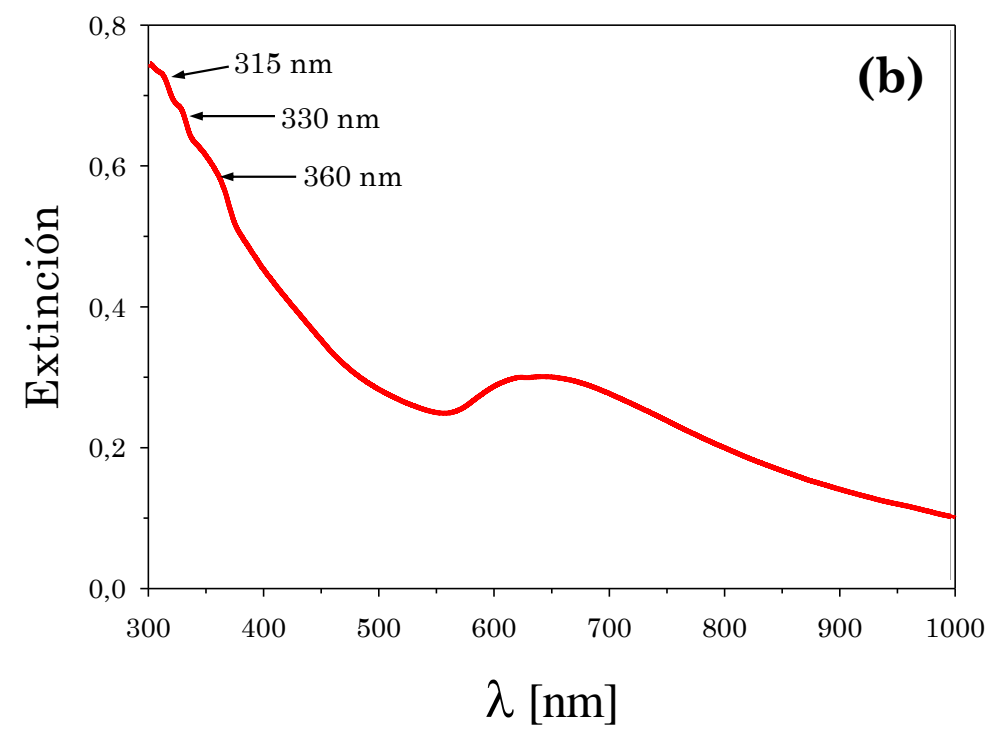

Figura 6.30: (a) Espectro experimental normalizado de una suspensión coloidal fabricada en agua con $100 \mu \mathrm{J}$ de energía por pulso. El ajuste teórico se obtuvo con nanopartículas de $\mathrm{Cu}_{2} \mathrm{O}-\mathrm{Cu}$ y $\mathrm{Cu}_{2} \mathrm{O}$. El recuadro muestra la distribución de tamaño óptimo de los radios del núcleo y espesor del shell que mejor se ajusta al espectro experimental. (b) Espectro experimental de la misma muestra donde se señalan los picos correspondientes a las transiciones de Brillouin del $\mathrm{Cu}_{2} \mathrm{O}$.

El ajuste del espectro experimental en la Figura 6.18 muestra que la suspensión coloidal fabricada con $500 \mu \mathrm{J}$ de energía contiene partículas de $\mathrm{Cu}-\mathrm{Cu}_{2} \mathrm{O}$ de radio subnanométrico, principalmente de $0,9 \mathrm{~nm}$ con dos espesores de óxido distintos de 40\% y 150\% del radio del núcleo, como se detalló en la Sección 6.1.2. Esta distribución angosta (de manera similar a la correspondiente para acetona con $500 \mu \mathrm{J}$ de energía por pulso), se puede interpretar en términos de las características particulares del proceso de fabricación a estas altas energías en el que las partículas son fabricadas y fragmentadas secuencialmente por absorción lineal.

El espectro obtenido con $100 \mu \mathrm{J}$ de energía en la Figura 6.30 también requiere atención especial. Durante el trabajo experimental, se observó que para una disminución secuencial de la energía, la cola de longitudes de onda cortas de los espectros disminuye su pendiente y el contraste se incrementa 
ligeramente. Para este caso, no fue posible encontrar un conjunto de parámetros que pudieran ajustar el espectro completo considerando solamente la configuración de $\mathrm{Cu}-\mathrm{Cu}_{2} \mathrm{O}$ utilizada para el agua a alta energía. Recordando los resultados obtenidos por Pike y coautores [Pike et al. 2006] mencionado anteriormente, las partículas de óxido de cobre pueden reducir a $\mathrm{Cu}$ cuando la temperatura aumenta. La temperatura a la que se produce esta transición de fase es dependiente del tamaño. Para partículas pequeñas (menos de $10 \mathrm{~nm}$ ) y temperaturas en el intervalo de 400 a $500{ }^{\circ} \mathrm{C}$, se forma una configuración de $\mathrm{Cu}_{2} \mathrm{O}-\mathrm{Cu}$. Para mayores temperaturas (superiores a $500{ }^{\circ} \mathrm{C}$ ) esta estructura reduce a $\mathrm{Cu}$ metálico. Dado que durante la ablación láser de femtosegundos estas temperaturas son de fácil acceso en el plasma generado en la pluma [König et al. 2005] y en la interfase plasma-líquido [Nath and Khare 2011] durante un intervalo de tiempo compatible con la cinética de oxidación rápida en la nanoescala (alrededor de $100 \mathrm{~ns}$ ), es posible sostener que, en virtud de ciertas condiciones experimentales, se generan en la suspensión coloidal nanopartículas con la configuración de $\mathrm{Cu}_{2} \mathrm{O}-\mathrm{Cu}$. Un núcleo de $\mathrm{Cu}_{2} \mathrm{O}$ cubierto por un shell de $\mathrm{Cu}$ metálico tiene un espectro de extinción diferente con respecto a la configuración inversa, lo cual se muestra en la Figura 6.31, donde se consideran los espectros de extinción calculados para diferentes especies $\left(\mathrm{Cu} \circ \mathrm{Cu}_{2} \mathrm{O}\right)$ en el núcleo de la estructura core-shell. Se puede observar que la configuración de $\mathrm{Cu}-\mathrm{Cu}_{2} \mathrm{O}$ para un núcleo de $3 \mathrm{~nm}$ de radio y $50 \%$ de espesor de $\mathrm{Cu}_{2} \mathrm{O}$ presenta la posición del pico cerca de $620 \mathrm{~nm}$, mientras que para una configuración de $\mathrm{Cu}_{2} \mathrm{O}-\mathrm{Cu}$ el pico del plasmón está centrado en $660 \mathrm{~nm}$ aproximadamente, mostrando un desplazamiento de $40 \mathrm{~nm}$ hacia el rojo. Además, la configuración anterior presenta una mayor pendiente en la cola de longitudes de onda cortas del espectro, en comparación con este último. 


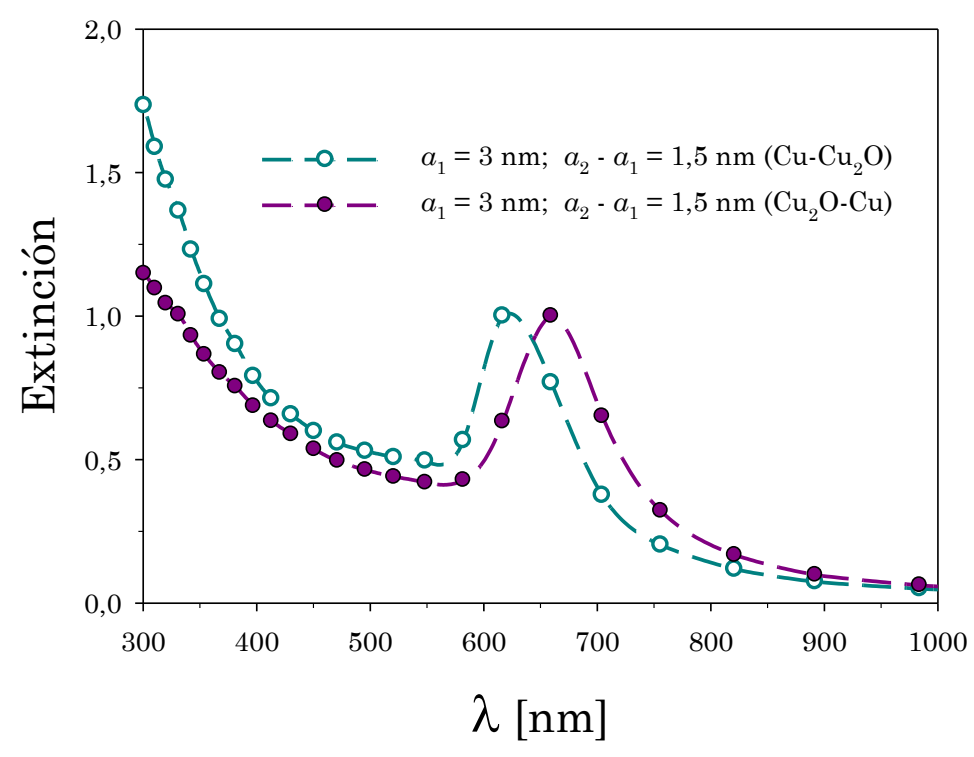

Figura 6.31: Espectros de extinción calculados para dos configuraciones core-shell diferentes: $\mathrm{Cu}-\mathrm{Cu}_{2} \mathrm{O}$ y $\mathrm{Cu}_{2} \mathrm{O}-\mathrm{Cu}$ con $3 \mathrm{~nm}$ de radio del núcleo y $50 \%$ de shell. El pico del plasmón de la segunda configuración muestra un desplazamiento hacia el rojo de $40 \mathrm{~nm}$ aproximadamente con respecto a la posición del plasmón de la primera, así como una pendiente menos pronunciada.

El corrimiento al rojo observado para la configuración que tiene shell de $\mathrm{Cu}$ metálico está de acuerdo con los resultados obtenidos por otros investigadores en las características plasmónicas de nanopartículas de núcleo dieléctrico-recubrimiento metal noble [Hirsch et al. 2003a, Tam et al. 2004]. Volviendo a la Figura 6.30 (a), el mejor ajuste se obtuvo con dos estructuras: una de ellas corresponde a nanopartículas de $\mathrm{Cu}_{2} \mathrm{O}-\mathrm{Cu}$ con un radio del núcleo dominante de $3 \mathrm{~nm}(60 \%)$ y diferentes espesores del shell, $30 \%$ de nanopartículas de $2 \mathrm{~nm}$ de radio del núcleo y una pequeña abundancia (menos de 5\%) de $5 \mathrm{~nm}$ de radio del núcleo. La otra estructura corresponde a nanopartículas de núcleo desnudo de $\mathrm{Cu}_{2} \mathrm{O}$ con un radio de $10 \mathrm{~nm}$. El cambio en la conformación de la estructura core-shell y la ampliación de la distribución de tamaños es más notable cuando disminuye la energía del láser. En este trabajo experimental, existe aparentemente un umbral de energía de $150 \mu \mathrm{J}$ aproximadamente en agua y $70 \mu \mathrm{J}$ en acetona 
debajo de la cual la configuración de $\mathrm{Cu}_{2} \mathrm{O}-\mathrm{Cu}$ domina por sobre la otra. Los resultados sobre la formación de nanopartículas con un núcleo de $\mathrm{Cu}_{2} \mathrm{O}$ generadas por ablación láser están en total acuerdo con los obtenidos por Liu y coautores [Liu et al. 2011], que trabajaron con un láser de pulsos de ns y una solución de PVP. La formación de una capa de cobre metálico alrededor de las nanopartículas de $\mathrm{Cu}_{2} \mathrm{O}$ se puede explicar en base a los resultados de otros autores [Amoruso et al. 2005, Gojo et al. 2005] acerca de la generación de nanopartículas metálicas en solución. Después de que un pulso láser de fs incide sobre la muestra, se produce en el plasma de una interfase sólido-líquido un aumento de presión y temperatura. La expansión adiabática que sigue este caso conduce a un enfriamiento rápido del plasma generado en la pluma y por lo tanto a la formación de clusters. Las nanopartículas se forman después de un retardo de tiempo de 5 a $30 \mu$ s aproximadamente [Amoruso et al. 2005], tiempo en el que la temperatura ha caído por debajo de $1000 \mathrm{~K}$ aproximadamente [Gojo et al. 2005]. Con la extinción del plasma, las nanopartículas formadas pueden interactuar con el oxígeno generado en el solvente por el pulso del láser, a partir de una reacción de oxidación que conduce a la componente de $\mathrm{Cu}_{2} \mathrm{O}$ de las nanopartículas core-shell. Esta oxidación puede ser completa o incompleta, principalmente en función de la concentración local de oxígeno atómico. Si la oxidación es completa, las nanopartículas de $\mathrm{Cu}_{2} \mathrm{O}$ generadas podrían alcanzar el siguiente pulso del láser, lo que aumentará su temperatura y producirá una reacción de reducción [Pike et al. 2006] que conduce a la formación de un recubrimiento de $\mathrm{Cu}$ metálico alrededor de las nanopartículas de $\mathrm{Cu}_{2} \mathrm{O}$.

Para comparar los resultados de la determinación de la composición de las partículas nanométricas y subnanométricas obtenidos con el método de espectroscopía de extinción óptica, se realizó un análisis de 
espectroscopía Raman de la suspensión coloidal generada con $500 \mu \mathrm{J}$ de energía por pulso en agua (Figura 6.18).

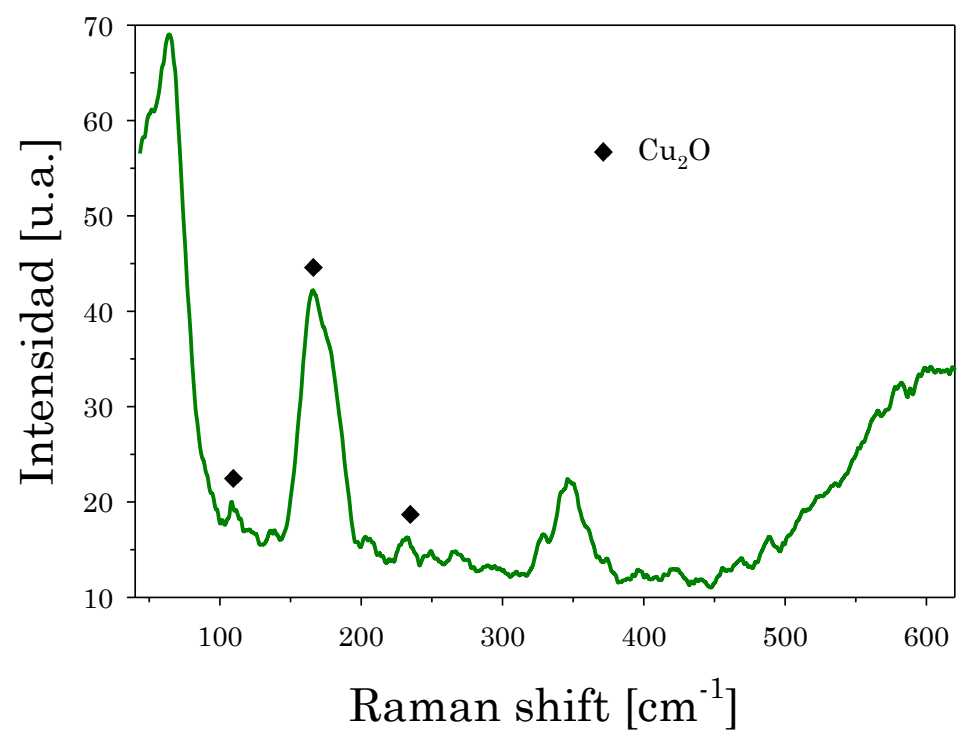

Figura 6.32: Espectro Raman experimental correspondiente a la suspensión coloidal generada en agua con $500 \mu \mathrm{J}$ de energía por pulso. Se observan tres picos característicos del $\mathrm{Cu}_{2} \mathrm{O}$ a $108 \mathrm{~cm}^{-1}, 165 \mathrm{~cm}^{-1}$ y $232 \mathrm{~cm}^{-1}$.

La Figura 6.32 muestra un espectro Raman obtenido experimentalmente como se describió en la Sección 5.3. En el espectro se observan tres picos característicos (rombos) del $\mathrm{Cu}_{2} \mathrm{O}$ que corresponden a los valores de $108 \mathrm{~cm}^{-1}\left(\Gamma_{12}\right), 165 \mathrm{~cm}^{-1}\left(\Gamma_{12}^{(1)}\right)$ y $232 \mathrm{~cm}^{-1}\left(\Gamma_{12}^{(-)}\right)$, los cuales están en muy buen acuerdo con los resultados obtenidos por Nath y Khare.

A fin de contrastar los resultados de la determinación de tamaños obtenidos a través del método de espectroscopía de extinción óptica, se realizó un análisis de microscopía de la muestra coloidal generada en agua usando ablación láser con una energía de $100 \mu \mathrm{J}$ por pulso (Figuras 6.30 (a)). 
La Figura 6.33 (a) muestra, a modo de ejemplo, una imagen AFM de la muestra coloidal generada en agua. Las partículas individuales se resolvieron con claridad por imágenes de AFM de un área de escaneo de $40 \mu \mathrm{m}$ x $40 \mu \mathrm{m}$ utilizando muestras de baja concentración.

Los perfiles de la altura de las líneas 1 a 6 de la Figura 6.33 (a) se representan en la Figura 6.33 (b). El escaneo de la primera línea en el rango de $8,3 \mu \mathrm{m}<x<10,8 \mu \mathrm{m}$ incluye una nanopartícula de $6,3 \mathrm{~nm}$ de altura. Este tamaño concuerda con los resultados obtenidos mediante espectroscopía de extinción óptica de la misma muestra, considerando una nanopartícula core-shell de $\mathrm{Cu}_{2} \mathrm{O}-\mathrm{Cu}$ con un radio del núcleo de $2 \mathrm{~nm}$ y un espesor del shell de 1,2 $\mathrm{nm}$, obteniéndose un radio externo de 3,2 $\mathrm{nm}$. La línea 2 de escaneo en el rango de $0 \mu \mathrm{m}<x<40 \mu \mathrm{m}$ contiene una nanopartícula de 6,8 nm de altura, la cual muestra un buen acuerdo con otro tamaño presente en la suspensión coloidal de $\mathrm{Cu}_{2} \mathrm{O}-\mathrm{Cu}$ de la Figura 6.30 (a), cuyo radio del núcleo es de $3 \mathrm{~nm}$ y el espesor del shell corresponde a un $13 \%$ de $a_{1}$ llegando a un radio externo de $3,4 \mathrm{~nm}$. En la línea 3 en el rango de $32 \mu \mathrm{m}<x<34 \mu \mathrm{m}$ se encuentra una nanopartícula de 7,3 $\mathrm{nm}$ de altura y concuerda con una partícula que corresponde al ajuste teórico de $a_{1}=3 \mathrm{~nm} \quad$ y $a_{2}=3,6 \mathrm{~nm}$. La línea 4 de escaneo en el rango de $15 \mu \mathrm{m}<x<17,5 \mu \mathrm{m}$ incluye una partícula de la solución coloidal de $7 \mathrm{~nm}$ de altura, la cual está en buen acuerdo con una nanopartícula de $a_{1}=3 \mathrm{~nm}$ y $a_{2}=3,45 \mathrm{~nm}$. En la línea 5 en el rango de $8,4 \mu \mathrm{m}<x<10,8 \mu \mathrm{m}$ se observó una nanopartícula cuya altura es de 13,4 nm, que se aproxima al valor de otro tamaño necesario para el ajuste teórico con un radio del núcleo de $3 \mathrm{~nm}$ y un espesor del shell del 120\% de $a_{1}$ obteniendo un radio externo de 6,6 nm. Por último, la línea 6 en el rango de 27,4 $\mu \mathrm{m}<x<29,6 \mu \mathrm{m}$ muestra una nanopartícula de 7,6 $\mathrm{nm}$ de altura, la cual se aproxima a un radio del núcleo de $3 \mathrm{~nm}$ con un espesor del shell de $0,9 \mathrm{~nm}$. 

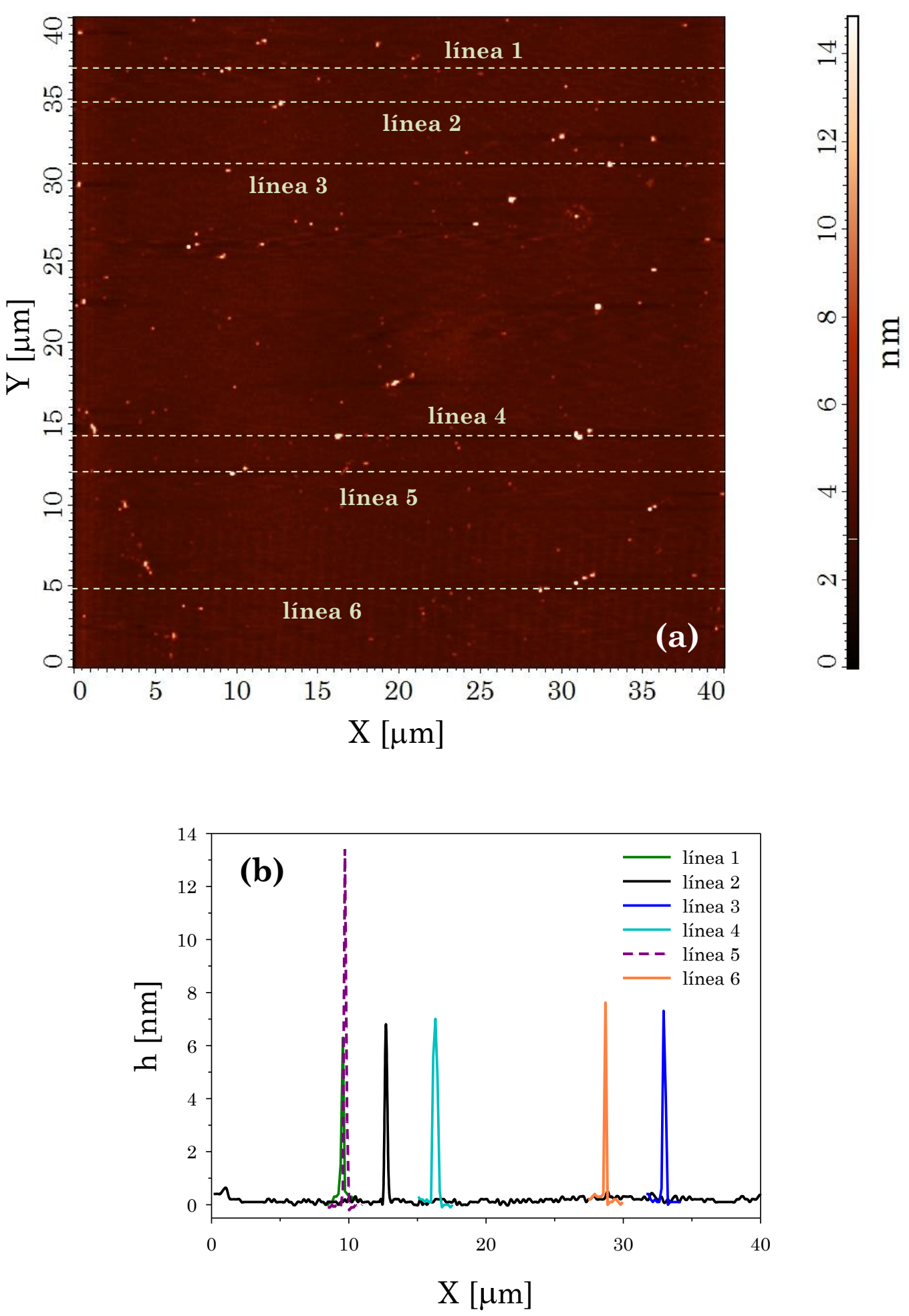

Figura 6.33: (a) Imagen AFM de la suspensión coloidal diluida obtenida por ablación láser con una energía de $100 \mu \mathrm{J}$ por pulso en agua. (b) Altura (diámetro) de las partículas nanométricas versus la posición X para las líneas 1 a 6 marcadas en la imagen del AFM. 
Estos resultados afirman nuevamente el hecho de que la espectroscopía de extinción óptica es una técnica muy sensible al tamaño de partículas nanométricas y subnanométricas de estructura simple y coreshell de las suspensiones coloidales. Los resultados arrojados por espectroscopía de extinción óptica son consistentes con los obtenidos a partir de técnicas estándar tales como HRTEM y AFM. 


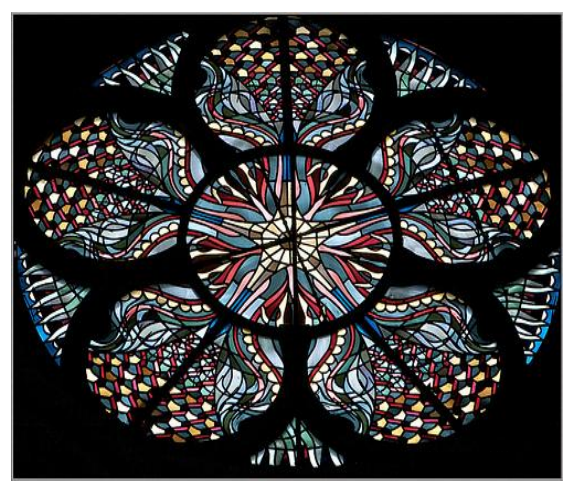

Catedral de Colonia

\section{Capítulo 7}

Colonia, Alemania

\section{Aplicaciones al sensado}

Las potenciales aplicaciones de los sensores basados en resonancia plasmónica son muy amplias sobre todo en áreas como la química y la biología, donde son utilizados para la detección de especies químicas en medios acuosos [Chau et al. 2006], detección de gases [Kim et al. 2007], pequeños cambios de índice de refracción [Homola 2008], velocidades de reacción [Ramakrishnan et al. 2005], entre otras. Estas aplicaciones son de alto interés no solo en el ámbito científico sino también en el industrial.

En el área biológica, existe una tendencia general a miniaturizar las arquitecturas de sensores con el fin de facilitar la detección simultánea de un gran número de antígenos diferentes en un único biochip, motivo por el cual las nanopartículas y los films delgados metálicos son las nanoestructuras con mayor crecimiento diseñadas para este desafío en los últimos años.

Numerosos trabajos desarrollados hasta el momento han mostrado la conveniencia de utilizar nanopartículas metálicas de tamaños inferiores a $20 \mathrm{~nm}$ de radio, debido a su mayor afinidad para la adsorción de moléculas biológicas sobre su superficie. A veces es necesario que un dieléctrico específico (anticuerpo en el caso de aplicaciones biológicas) recubra el núcleo 
de una nanopartícula metálica o que forme el núcleo de una estructura coreshell (dieléctrico-metal) [Hirsch et al. 2003a, Prodan et al. 2003], para lograr una sintonía adecuada de la resonancia del plasmón.

La detección de pequeñas cantidades de moléculas de proteínas adsorbidas en la superficie de nanopartículas de plata a través del conteo de las mismas en forma aislada en base a imágenes de microscopía de campo oscuro ha sido reportada por Schultz y colaboradores [Schultz et al. 2000].

En este Capítulo se presentan las aplicaciones al diseño de dos tipos de sensores plasmónicos: uno de ellos, de partícula aislada de plata, y el otro de películas delgadas de plata basado en la configuración de Kretschmann. En ambos casos el sensado de oxígeno se realiza a través de la medición del espesor de óxido de plata crecido sobre la nanoestructura de plata.

En el primer caso, se analiza el crecimiento del óxido de plata alrededor de una partícula aislada de plata a través del comportamiento de determinados parámetros plasmónicos característicos del espectro de extinción óptico de la partícula, permitiendo establecer un simple protocolo para determinar el tamaño del radio del núcleo y el espesor del recubrimiento de óxido.

Respecto al segundo caso, se utiliza la configuración de Kretschmann (descripta en la Sección 4.3), para determinar el espesor del óxido de plata crecido sobre una película delgada de plata, mediante el análisis de la reflectividad de la multicapa de plata-óxido de plata para polarizaciones de onda $p$ y $s$. 


\subsection{Partícula aislada de plata-óxido de plata}

Para evitar posibles perturbaciones en la resonancia del plasmón debido a la interacción conjunta de las nanopartículas en una suspensión coloidal, en los últimos años, muchos trabajos han analizado las medidas de la extinción de partícula aislada. Estos se basan generalmente en espectros de scattering utilizando microscopía de campo oscuro [Novo et al. 2008] o de absorción empleando una nueva técnica llamada espectroscopía de modulación espacial [Billaud et al. 2007, Muskens et al. 2008, Baida et al. 2009], en ambos casos de partícula única. Estas aplicaciones están orientadas al desarrollo de nanosensores en diferentes campos, tales como fotocatálisis [Grady et al. 2004], adsorción química en la nanoescala [McFarland and Van Duyne 2003] y sensado de compuestos biológicos [Prodan et al. 2003, Anker et al. 2008].

Las propiedades ópticas de nanopartículas aisladas tipo core-shell, por lo general, presentan una combinación de las características de ambos materiales del núcleo y del recubrimiento, muchas veces en una manera distintiva. Algunos trabajos estudian partículas core-shell formadas por un núcleo metálico y un óxido de otro metal como recubrimiento [Baida et al. 2009]. Sin embargo, existen pocos trabajos de nanopartículas core-shell con un núcleo metálico y su propio óxido como recubrimiento. Valverde-Aguilar y coautores [Valverde-Aguilar et al. 2008] estudiaron la formación de partículas metálicas de plata en el rango de tamaños nanométricos en una matriz de $\mathrm{SiO}_{2}$ por reducción de $\mathrm{AgNO}_{3}$. Las imágenes TEM muestran una distribución aleatoria de nanopartículas de plata exhibiendo un recubrimiento de óxido de plata alrededor de ellas. El espectro de extinción experimental de las nanopartículas presenta una banda de absorción situada en $438 \mathrm{~nm}$. Ghilane y coautores [Ghilane et al. 2007] analizaron la formación de un recubrimiento de $\mathrm{Ag}_{2} \mathrm{O}$ en un núcleo de $\mathrm{Ag}$ usando un 
tratamiento térmico de nanopartículas de $\mathrm{Ag}$ a temperaturas entre $200 \mathrm{y}$ $360{ }^{\circ} \mathrm{C}$ en fase gaseosa y en solvente orgánico. Los autores caracterizaron las nanopartículas antes formadas por voltametría cíclica. Los diámetros típicos eran menores que $30 \mathrm{~nm}$. Karavanskii y coautores [Karavanskii et al. 2004] proponen la formación de un recubrimiento de óxido de plata alrededor de nanopartículas de plata para explicar las propiedades ópticas no lineales de suspensiones coloidales obtenidas por ablación láser de un blanco sólido en agua. En algunos de estos trabajos, los espectros de partícula aislada son ajustados usando la aproximación electrostática de la teoría de Mie [Valverde-Aguilar et al. 2008], o simplemente un ajuste Lorentziano [Novo et al. 2009]. Estos trabajos conllevan una creciente necesidad al desarrollo de diferentes métodos para caracterizar el tamaño del núcleo y espesor del recubrimiento de las nanopartículas.

\subsubsection{Sensor de partícula aislada de Ag-óxido de Ag}

Debido a la gran necesidad de caracterizar tamaños de nanopartículas metálicas, se desarrolló un método paramétrico para la determinación del radio del núcleo y espesor del recubrimiento de pequeñas nanopartículas core-shell de plata-óxido de plata, basado en espectroscopía de extinción óptica de partícula aislada.

El método se basó en el estudio de la relación entre la longitud de onda del pico del plasmón, el ancho total a altura media (FWHM, Full Width at Half Maximum), y el contraste, definido como $\left(I_{\text {máx }}-I_{\text {mín }}\right) / I_{\text {máx }}$, de los espectros de extinción en función del radio del núcleo y espesor del recubrimiento.

Usando la aproximación electrostática de la teoría de Mie (ecuación (2.33)) junto con la expresión de la polarizabilidad para partículas de 
estructura core-shell (ecuación (2.34)), se calcularon los espectros de extinción de partículas aisladas de $\mathrm{Ag}-\mathrm{Ag}_{2} \mathrm{O}$ para $a_{1} \leq 20 \mathrm{~nm}$ y diferentes espesores de $\mathrm{Ag}_{2} \mathrm{O}$.

Los espectros de extinción de nanopartículas aisladas de estructura core-shell son muy sensibles a pequeños cambios en el espesor del recubrimiento como se puede observar en la Figura 7.1 (a), donde se representan espectros de extinción óptica calculados para nanopartículas de estructura simple con un radio de 3 y $7 \mathrm{~nm}$, y de estructura núcleorecubrimiento con el mismo tamaño de radio y un espesor del recubrimiento de $10 \% a_{1}$. Los datos del índice de refracción del $\mathrm{Ag}_{2} \mathrm{O}$ fueron tomados de Qiu y coautores [Qiu et al. 2005] y están representados en la Figura 7.1 (b).

Aunque el espectro de extinción de $7 \mathrm{~nm}$ es en realidad más grande que el de $3 \mathrm{~nm}$, cada espectro ha sido normalizado en su máximo para propósitos de comparación. Se pueden notar tres aspectos: 1) la longitud de onda de los picos del plasmón se desplaza hacia el rojo con pequeños incrementos de espesores de $\mathrm{Ag}_{2} \mathrm{O}$ y parece ser casi independiente del radio del núcleo, 2) el contraste (donde $I_{\min }$ se toma alrededor de $\lambda_{\min }=312 \mathrm{~nm}$ ) se incrementa con radios del núcleo y espesores del recubrimiento y 3) el FWHM de las curvas de resonancia disminuye a medida que aumenta el radio del núcleo, mientras que incrementa en función del espesor del óxido. Este último comportamiento del FWHM se debe a la existencia de una parte imaginaria no despreciable en la función dieléctrica del óxido de plata (shell) (relacionado con la pérdida de energía [Kreibig and Vollmer 1995, Bohren and Huffman 1998]), que produce una ampliación y una reducción de la altura de la resonancia del plasmón a medida que aumenta el espesor del óxido. El comportamiento general de estas relaciones puede ser representado si la longitud de onda del pico del plasmón, el contraste $(C)$ y el FWHM se grafican en función del radio del núcleo y espesor del recubrimiento. 

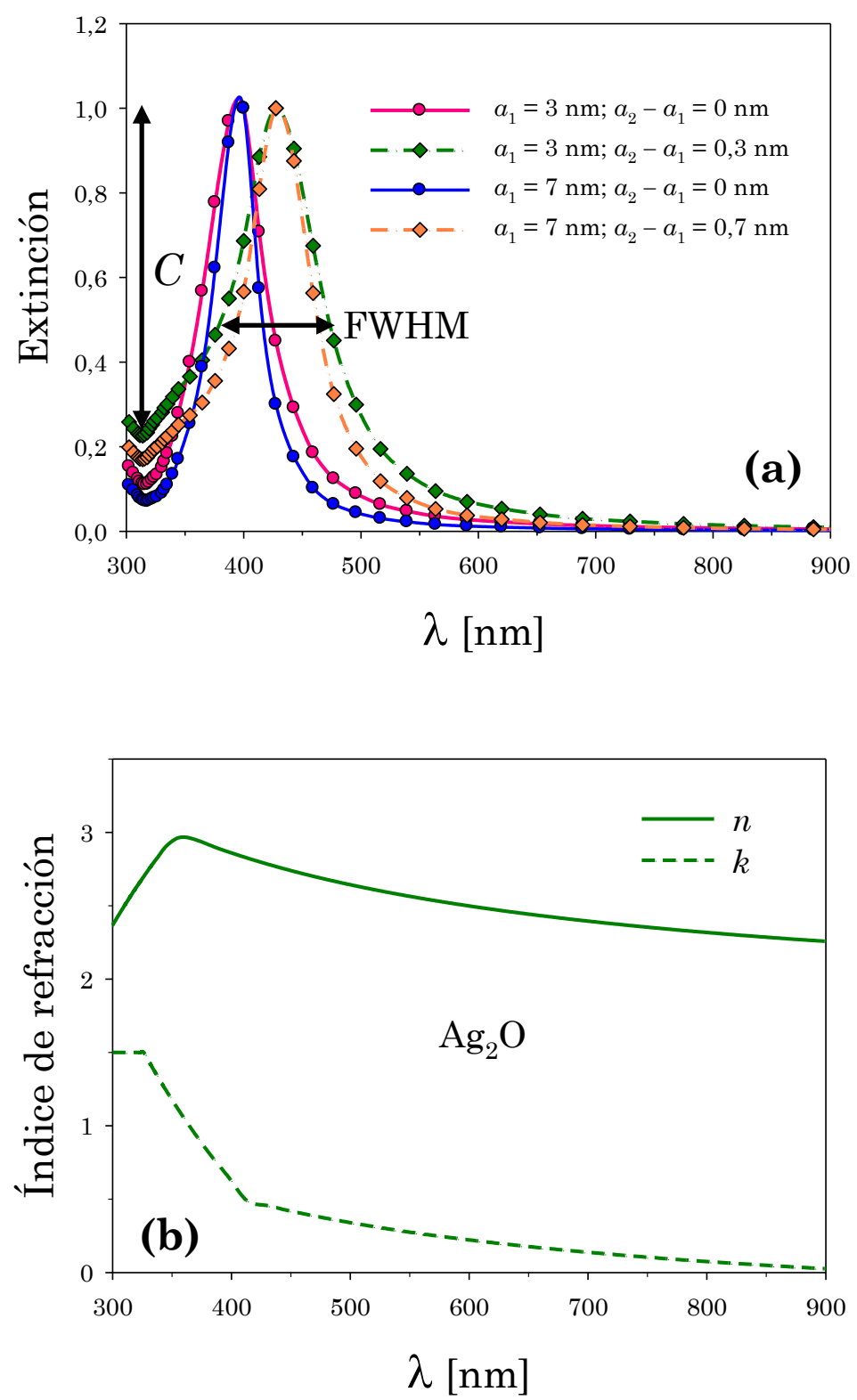

Figura 7.1: (a) Coeficiente de extinción normalizado de nanopartículas de plata con y sin recubrimiento de $\mathrm{Ag}_{2} \mathrm{O}$ calculado para diferentes radios y espesores. (b) Índice de refracción de $\mathrm{Ag}_{2} \mathrm{O}$ utilizado para los cálculos teóricos.

La Figura 7.2 muestra gráficos 3D de estas relaciones para valores discretos de las últimas variables usando agua $(n=1,33)$ como medio circundante. Se puede observar que, mientras el pico del plasmón parece tener una fuerte dependencia con espesores del recubrimiento y una débil 
dependencia con radios del núcleo (Figura 7.2 (a)), el contraste y el FWHM dependen de ambas variables simultáneamente (Figura 7.2 (b) y (c), respectivamente).
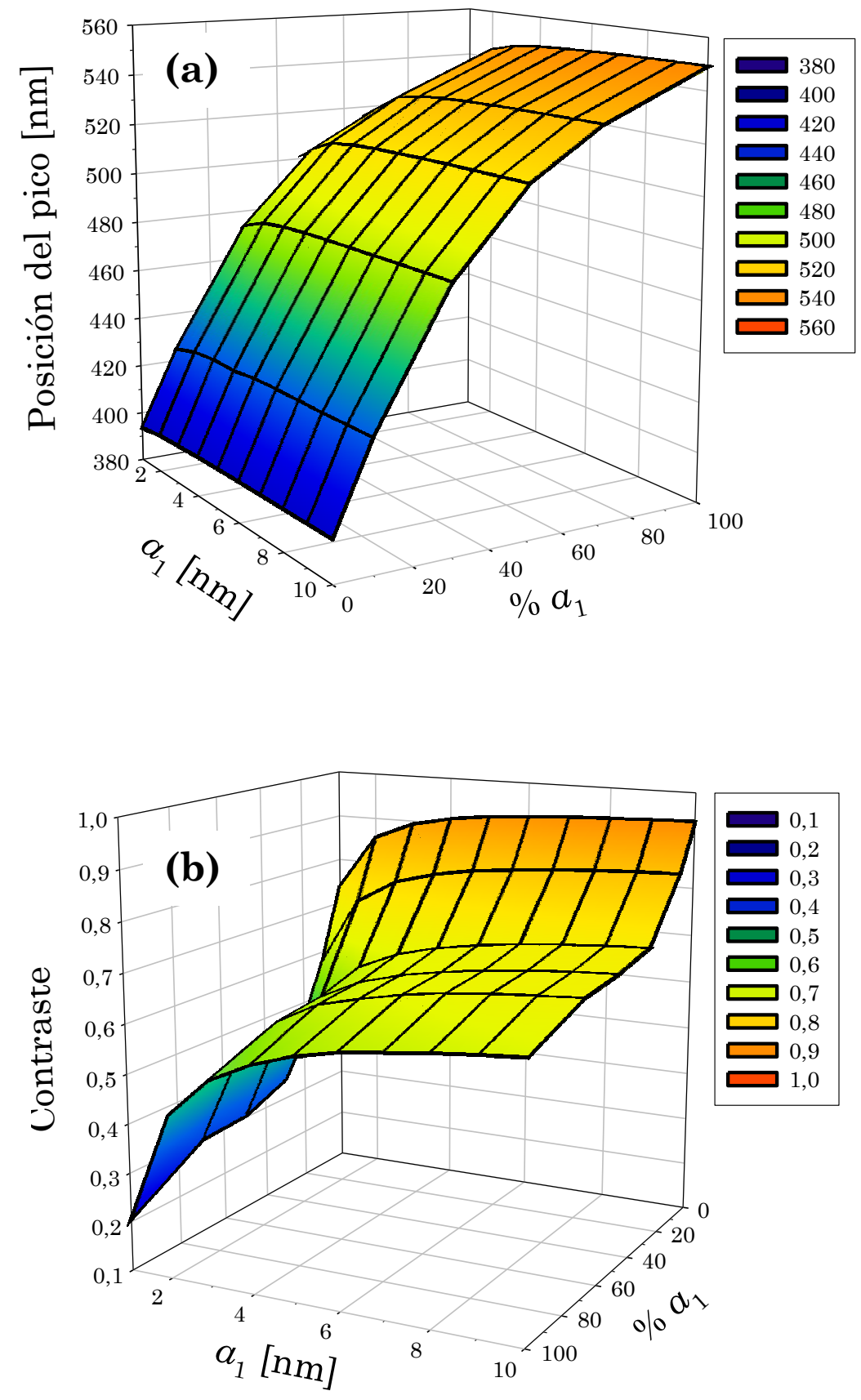


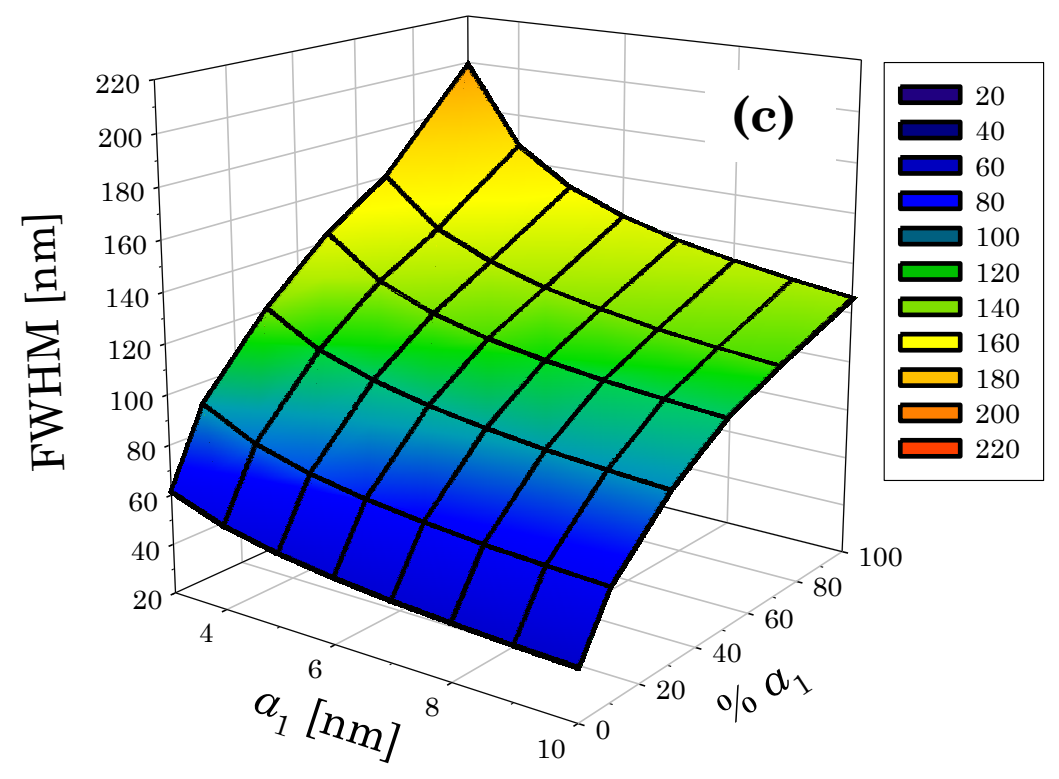

Figura 7.2: (a) Longitud de onda del pico del plasmón, (b) contraste y (c) FWHM en función del radio del núcleo y espesor del recubrimiento para nanopartículas de $\mathrm{Ag}-\mathrm{Ag}_{2} \mathrm{O}$ en agua.

Esta doble dependencia se debe al hecho de que el índice de refracción de $\mathrm{Ag}_{2} \mathrm{O}$ (Figura 7.1 (b)), tiene un término imaginario que representa la pequeña absorción de este material en función de la longitud de onda [Qiu et al. 2005]. Esta característica sugiere que puede llevarse a cabo un estudio más detallado del comportamiento de cada uno de estos parámetros con el radio del núcleo y el espesor del recubrimiento para determinar los tamaños de las nanopartículas de una manera simple y rápida.

De manera inversa, estos cálculos pueden ayudar a diseñar una nanopartícula especifica de $\mathrm{Ag}-\mathrm{Ag}_{2} \mathrm{O}$, de tal forma que su pico del plasmón este centrado a una determinada longitud de onda, usando solo información óptica. A su vez, el espesor del recubrimiento puede estar relacionado con el contenido de oxígeno en los medios que rodea la nanopartícula. 

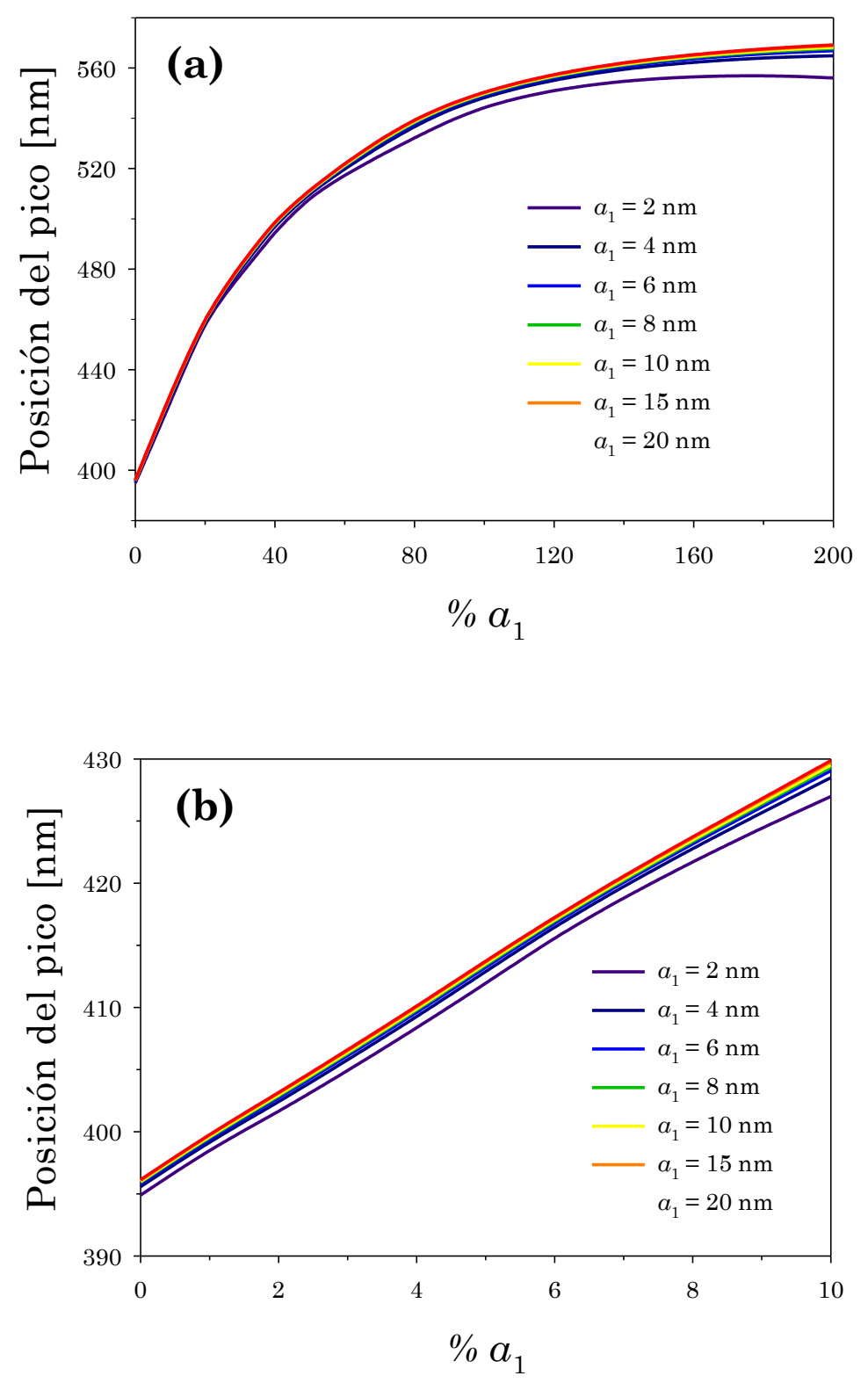

Figura 7.3: Posición del pico del plasmón de nanopartículas esféricas de $\mathrm{Ag}-\mathrm{Ag}_{2} \mathrm{O}$ en función del espesor del shell con el radio del núcleo como parámetro: (a) rango completo del espesor del shell que va de 0 a $200 \% a_{1}$ y (b) rango reducido del espesor del shell que va de 0 a $10 \% a_{1}$.

La Figura 7.3 (a) muestra el comportamiento de la posición del pico en función del espesor del recubrimiento luego de un análisis minucioso para 
diferentes radios del núcleo de plata (2 a $20 \mathrm{~nm}$ ) y espesores del recubrimiento que van de 0 a $200 \% a_{1}$.

Se puede notar que todas las curvas correspondientes a los radios del núcleo entre 2 y $20 \mathrm{~nm}$ son muy cercanas entre sí, casi independientes del tamaño del núcleo. Dos regiones pueden ser claramente distinguidas: una casi lineal, hasta un espesor del $20 \% a_{1}$, y una no lineal por encima de este mismo valor de porcentaje, mostrando un comportamiento de saturación para espesores muy grandes. Pequeñas variaciones del espesor del recubrimiento producen cambios medibles en la posición del pico, excepto en el régimen de saturación. Este resultado coincide con uno similar obtenido por Evanoff y coautores [Evanoff et al. 2004] al estudiar nanopartículas de $\mathrm{Ag}$ - $\mathrm{SiO}_{2}$, donde la longitud de onda de la resonancia del plasmón aumenta constantemente con el espesor del shell hasta aproximadamente la mitad del radio, después del cual la relación disminuye y el desplazamiento alcanza la saturación para una distancia aproximada al valor del radio de las nanopartículas. Este comportamiento está relacionado a la extensión espacial del campo electromagnético asociado con la oscilación de la densidad de carga coherente (plasmón) [Evanoff et al. 2004]. Para el caso del $\mathrm{Ag}_{2} \mathrm{O}$, la región lineal muestra una pendiente empinada $(70 \mathrm{~nm}$ de corrimiento / $6 \mathrm{~nm}$ de espesor) en comparación con una capa dieléctrica de $\mathrm{SiO}_{2}(25 \mathrm{~nm}$ de corrimiento / $20 \mathrm{~nm}$ de espesor) obtenida por Evanoff y coautores. Por lo tanto, el análisis de la posición del pico del plasmón permitió determinar un estrecho intervalo de porcentajes de espesores del recubrimiento, independientemente del radio del núcleo. En la Figura 7.3 (b) se representa una ampliación detallada de la región lineal para el rango de 0 a $10 \% a_{1}$ de espesor del recubrimiento. Esta dependencia casi lineal de la posición del pico del plasmón con el espesor del recubrimiento puede ser la base para detectar el espesor del óxido de plata, que está relacionado con la 
cantidad de oxígeno presente en la vecindad inmediata de la partícula, como se mostrará más adelante.

La Figura 7.4 muestra el comportamiento del contraste $C$ en función del radio del núcleo en el intervalo de 1 a $20 \mathrm{~nm}$, dejando el espesor como un parámetro.
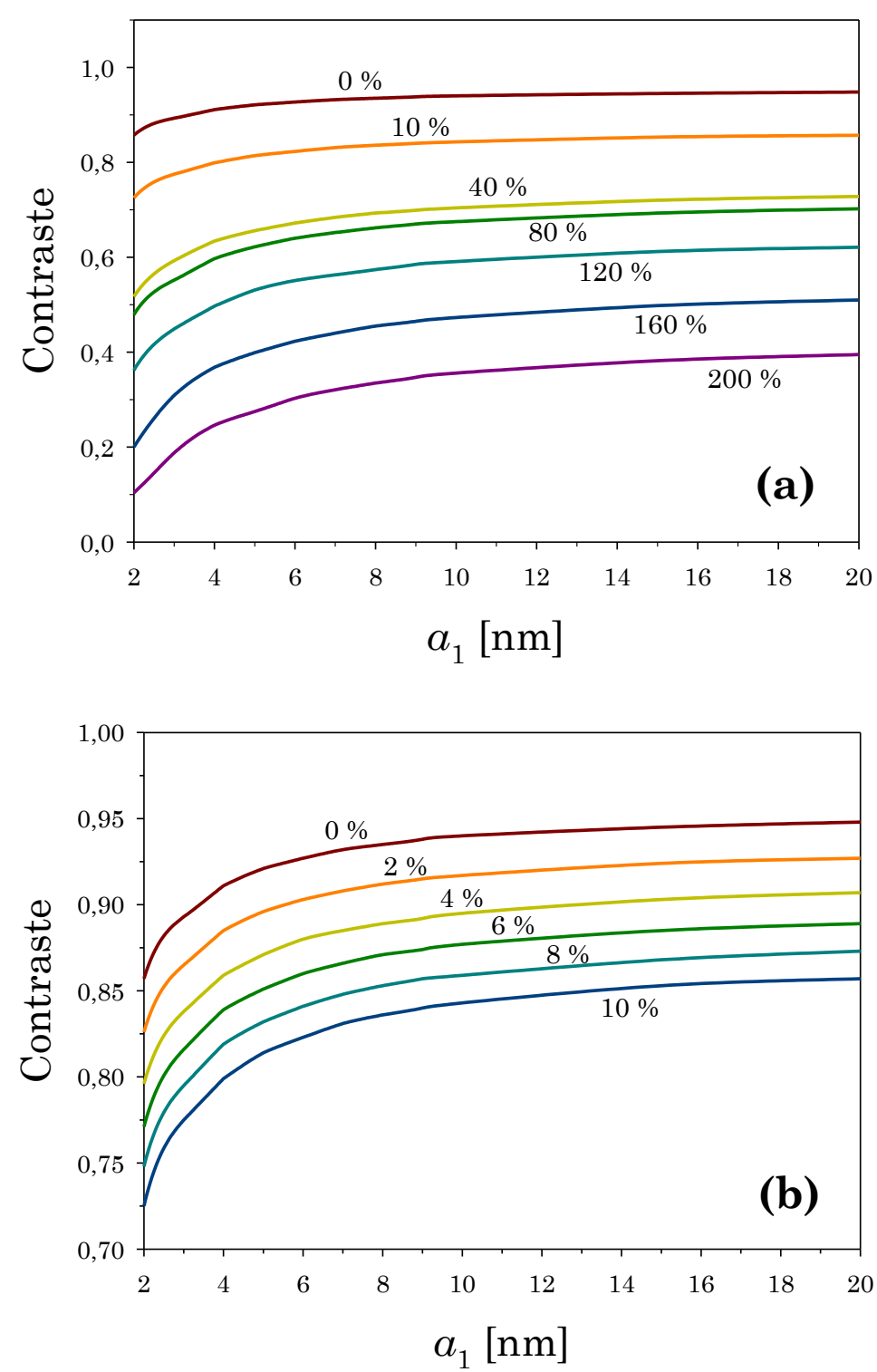

Figura 7.4: Contraste del espectro de extinción de nanopartículas esféricas de $\mathrm{Ag}-\mathrm{Ag}_{2} \mathrm{O}$ en función del radio del núcleo con el espesor del recubrimiento como parámetro: (a) rango completo del espesor del shell que va de 0 a $200 \% \quad a_{1}$ y (b) rango reducido del espesor del shell que va de 0 a $10 \% a_{1}$. 
De la Figura 7.4 (a) se puede ver que el contraste es una función monótonamente creciente del radio del núcleo para el rango de espesores completo ( 0 a $200 \% a_{1}$ ), mientras que su valor disminuye a medida que aumenta el espesor. La Figura 7.4 (b) muestra el contraste en un rango de espesor reducido ( 0 a $10 \% a_{1}$ ), donde es posible observar cambios notables para radios por debajo de $10 \mathrm{~nm}$. Para radios mayores que este valor, el contraste tiende a un valor aproximadamente constante para cada espesor del recubrimiento. Por lo tanto, el análisis del contraste en los espectros de extinción permitió caracterizar el radio desnudo, una vez conocido el espesor.

Finalmente, la Figura 7.5 (a) muestra el comportamiento del FWHM en función del radio del núcleo $a_{1}$ para diferentes espesores del recubrimiento entre 0\% para una nanopartícula de núcleo desnudo y $100 \%$ $\left(a_{2}=2 a_{1}\right)$. Se puede notar que, para un determinado valor de $a_{1}$, el FWHM aumenta monótonamente con el espesor del recubrimiento. Por otra parte, se puede observar que, para un dado espesor del recubrimiento, el FWHM disminuye al aumentar el radio del núcleo.

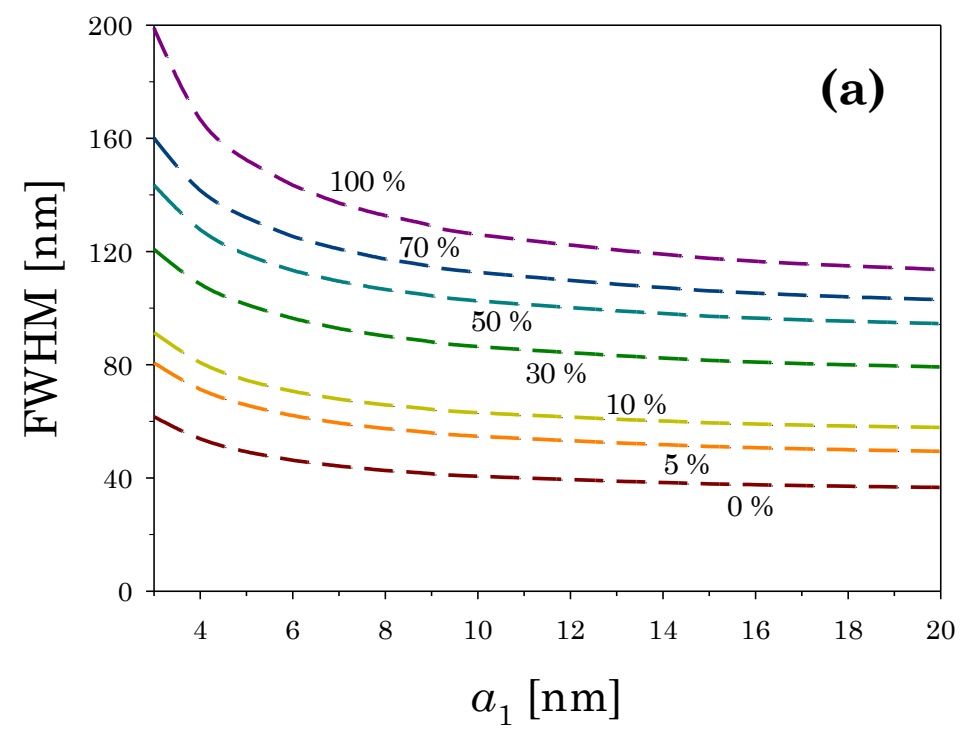




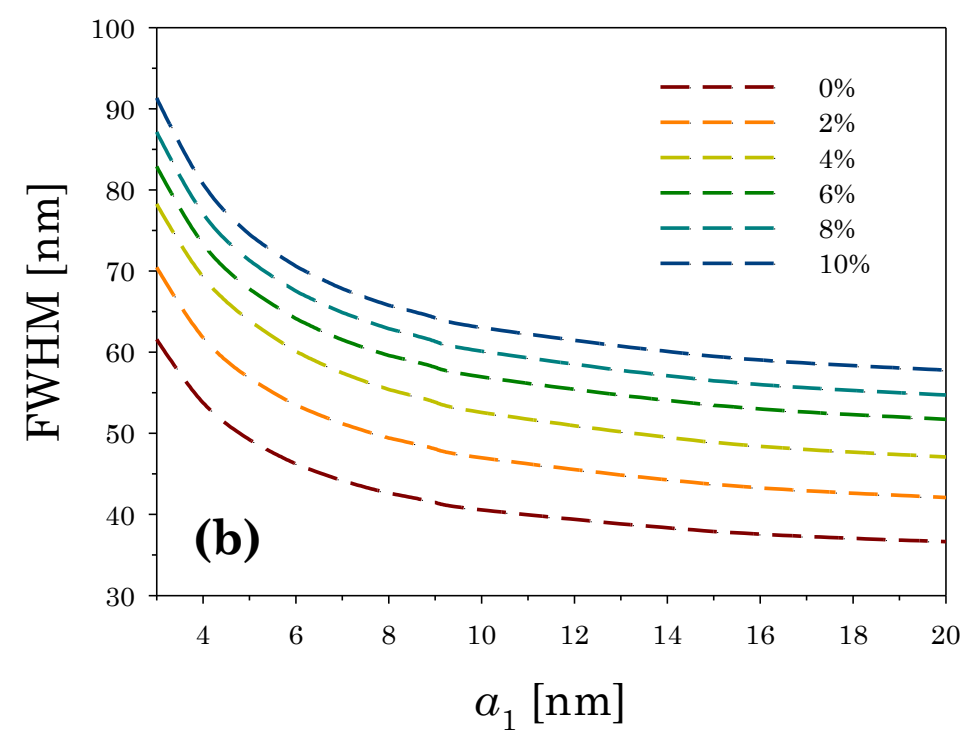

Figura 7.5: FWHM del espectro de extinción de nanopartículas esféricas de $\mathrm{Ag}-\mathrm{Ag}_{2} \mathrm{O}$ en función del radio del núcleo con el espesor del recubrimiento como parámetro: (a) rango completo del espesor del shell que va de 0 a $200 \% a_{1}$ y (b) rango reducido del espesor del shell que va de 0 a $10 \% a_{1}$.

En la Figura 7.5 (b) se presenta de una manera más detallada el comportamiento del FWHM con radios del núcleo para un rango de espesores del 0 a $10 \% a_{1}$.

\subsubsection{Diseño y aplicación del método paramétrico}

En base a las características de los espectros de extinción óptica que se mostraron en las figuras anteriores, se ha desarrollado un procedimiento paso a paso calibrado para medir el espesor del recubrimiento de óxido en una nanopartícula aislada [Santillán et al. 2011], que se puede resumir de la siguiente manera: 
1- Medir la longitud de onda de la resonancia (posición del pico del plasmón) de los espectros de extinción experimentales.

2- Con este valor, determinar el espesor del recubrimiento de óxido (en unidades de porcentaje del radio del núcleo) de las curvas de la Figura 7.3.

3- Medir el contraste $C$ (o el FWHM, lo que se pueda medir con una precisión razonable) del espectro de extinción experimental.

4- Con este valor, determinar el radio del núcleo por la intersección entre la ordenada del contraste (o del FWHM) y la curva del porcentaje de recubrimiento correspondiente utilizando la Figura 7.4 (o la Figura 7.5, respectivamente).

Como una aplicación a este método paramétrico, se analizaron los datos de extinción experimental de una nanopartícula aislada de plata tomados de Billaud y colaboradores [Billaud et al. 2007], quienes utilizaron el método de espectroscopía de modulación espacial para registrar el espectro de absorción en diferentes medios. El espectro corresponde a una nanopartícula de un diámetro medio de $40 \mathrm{~nm}$ obtenida por spin coating sobre alúmina porosa.

Debido a que las nanopartículas de plata pueden haber reaccionado con el oxígeno en la solución dispersada por spin coating o en el medio de inclusión, formándose una capa delgada de óxido de plata alrededor del núcleo de plata, el ajuste del espectro experimental se realizó considerando que la nanopartícula posee una estructura core-shell de $\mathrm{Ag}-\mathrm{Ag}_{2} \mathrm{O}$.

La posición del pico del plasmón del espectro de extinción experimental (representado por círculos en la Figura 7.6) se encuentra en $411 \mathrm{~nm}$. Analizando las curvas mostradas en la Figura 7.3 (b), se puede concluir que el porcentaje del espesor óptimo de $\mathrm{Ag}_{2} \mathrm{O}$ es de $4 \% \pm 0,2 \%$ de 
$a_{1}$ aproximadamente. Siguiendo el procedimiento paso a paso descripto anteriormente, a partir del FWHM del espectro experimental $(\Delta \lambda=46,5 \mathrm{~nm})$, se puede deducir que el radio del núcleo $a_{1}$ está alrededor de $21 \mathrm{~nm} \pm 2 \mathrm{~nm}$. Para verificar la precisión del método, se realizó un ajuste del espectro completo en los puntos experimentales.

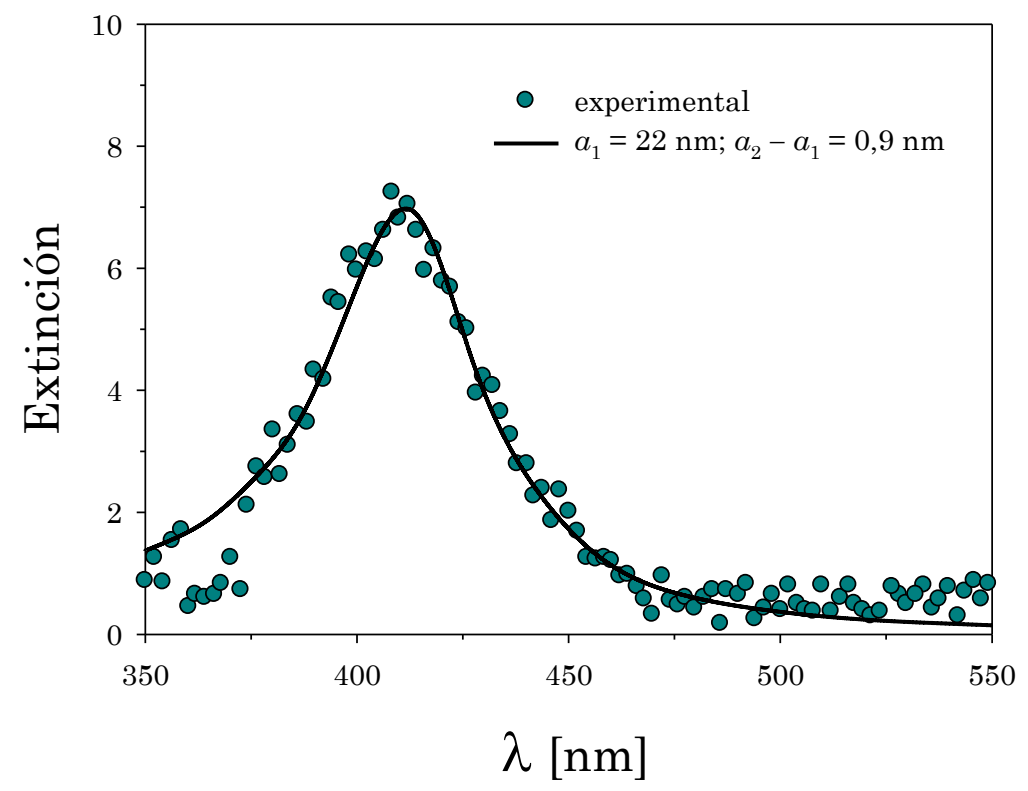

Figura 7.6: Espectro de extinción teórico (línea sólida) de nanopartícula aislada de $\mathrm{Ag}-\mathrm{Ag}_{2} \mathrm{O}$. El mejor ajuste de los puntos experimentales (círculos) corresponde a una nanopartícula core-shell con $a_{1}=22 \mathrm{~nm}$ y $a_{2}=22,9 \mathrm{~nm}$ [Santillán et al. 2011].

La Figura 7.6 muestra también el mejor ajuste (línea sólida) de los datos experimentales considerando una nanopartícula de $\mathrm{Ag}-\mathrm{Ag}_{2} \mathrm{O}$ con $a_{1}=22 \mathrm{~nm}$ y $a_{2}=22,9 \mathrm{~nm}$, usando datos experimentales de la función dieléctrica bulk de plata y considerando un medio con índice de refracción similar al utilizado en [Billaud et al. 2007] ( $n=1,33)$. Existe un excelente acuerdo entre los puntos experimentales y la curva de ajuste, obteniéndose valores del radio del núcleo y del espesor del recubrimiento aproximados a los determinados por el método paramétrico. 
Por comparación, en la Figura 7.7 (a) se muestra un espectro de extinción calculado (línea discontinua) de una nanopartícula de núcleo desnudo de Ag con el mismo radio del núcleo y en el mismo medio, además de los datos experimentales y el mejor ajuste.
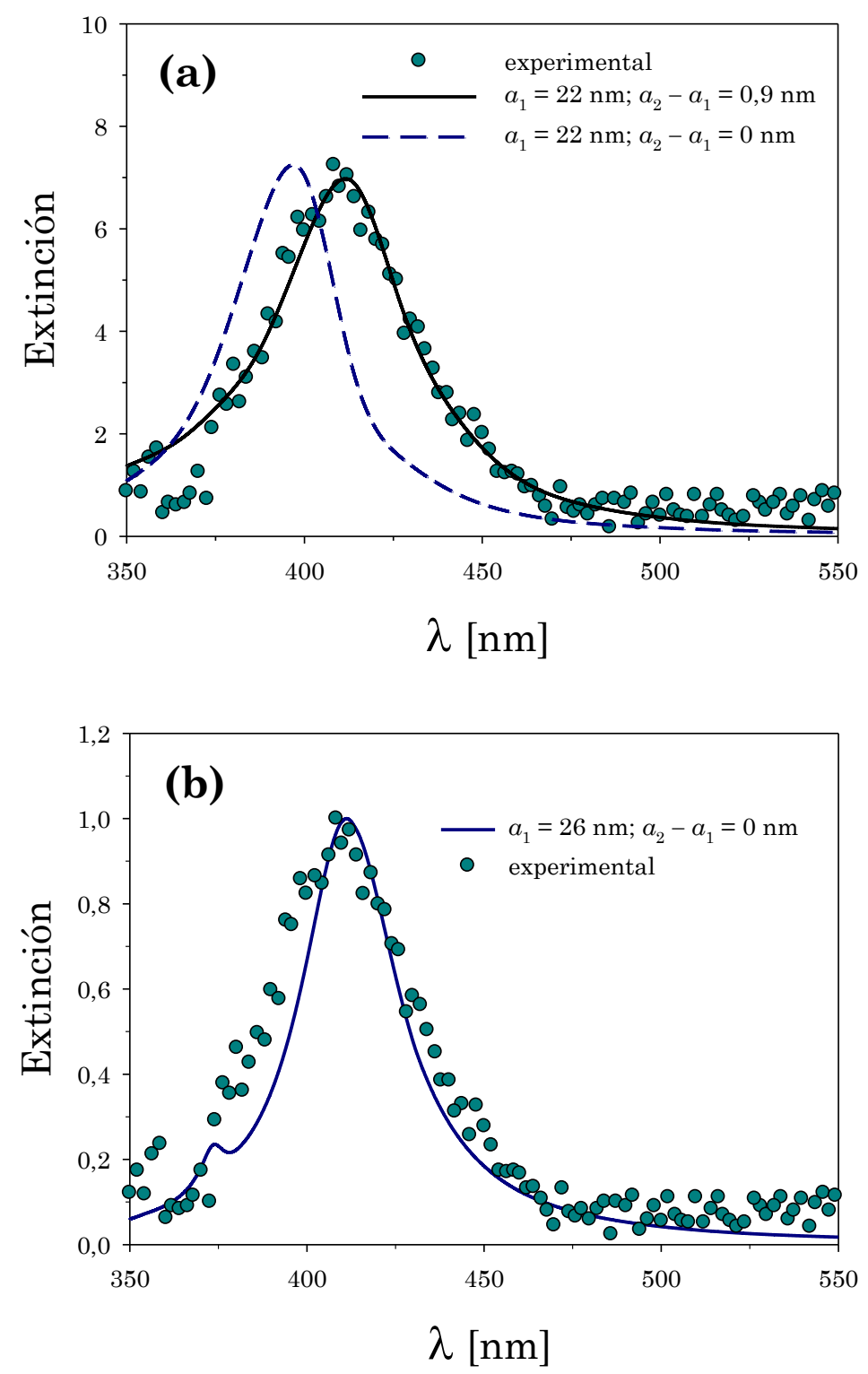

Figura 7.7: (a) Comparación de un espectro de extinción teórico (línea discontinua) de nanopartícula aislada de Ag de estructura simple de $a_{1}=22 \mathrm{~nm}$ con el mejor ajuste (línea sólida) y los datos experimentales (círculos). (b) Cálculos de Mie de una nanopartícula de Ag de estructura simple con $a_{1}=26 \mathrm{~nm}$ que coincide con la longitud de onda del pico del plasmón del espectro de extinción experimental, pero no reproduce el FWHM. 
Se puede ver que existe una discrepancia tanto en la longitud de onda del pico del plasmón como en el FWHM. La posición del pico de la nanopartícula de núcleo desnudo está desplazada unos $20 \mathrm{~nm}$ hacia el azul. Link y El-Sayed [Link and El-Sayed 2000] afirman que la aproximación electrostática puede utilizarse de manera confiable para tamaños de radio alrededor de $20 \mathrm{~nm}$ aproximadamente. El posible error introducido en el cálculo utilizando esta aproximación para un radio de $22 \mathrm{~nm}$ está incluido en el intervalo de 1 a $2 \%$.

Para apoyar los resultados del mejor ajuste, se representa en la Figura 7.7 (b) el espectro teórico normalizado de una nanopartícula aislada de Ag de estructura simple con $a_{1}=26 \mathrm{~nm}$, utilizando el cálculo de Mie (ya que para este tamaño, la aproximación electrostática no es válida) para un índice de refracción del medio de 1,33. Aunque la longitud de onda del pico se reproduce, el FWHM no coincide con los datos experimentales. Además, se pueden observar diferencias notables en el espectro teórico debido a la contribución cuadrupolar cerca de $375 \mathrm{~nm}$. Por lo tanto, se puede concluir que nanopartículas de estructura simple de este tamaño de radio no se ajustan a los espectros experimentales. Sin embargo, una nanopartícula de núcleo de plata y recubrimiento de óxido de plata puede reproducir un mejor ajuste, considerando un radio del núcleo y un espesor del recubrimiento apropiados.

\subsection{Películas nanométricas de $\mathrm{Ag}$ y $\mathrm{Ag}_{2} \mathrm{O}$}

Existe una necesidad constante de medición de espesores de películas nanométricas para una amplia variedad de aplicaciones industriales y científicas principalmente por sus propiedades ópticas y eléctricas [Smith et 
al. 1997, Hou et al. 1998, Libardi and Grieneisen 1998, Fuji et al. 2000, Peyser et al. 2001, Fujimaki et al. 2006].

Las películas delgadas de óxido de plata han sido utilizadas en la industria fotográfica y también para aplicaciones eléctricas (electrodos de baterías). En el área de la optoelectrónica, es un componente importante en la superficie del fotocátodo tipo S-1 para fotomultiplicadores de respuesta rápida en el visible-IR cercano [Hou et al. 1998], así como en el área de almacenamiento de datos magneto-ópticos [Smith et al. 1997], scattering Raman reforzado por efecto de superficie (SERS, Surface-Enhanced Raman Scattering) en dispositivos plasmónicos y super resolución (super-RENS, super-Resolution Near-Field Structure), con técnicas de campo cercano en estructuras plasmónicas utilizadas para resolver señales pequeñas producidas por partículas de tamaño más pequeño que el límite de difracción [Fuji et al. 2000, Peyser et al. 2001, Fujimaki et al. 2006]. Hay muchos trabajos que tratan el estudio del crecimiento de óxido de plata en diferentes substratos bajo diferentes condiciones experimentales [Libardi and Grieneisen 1998, Gao et al. 2004, Sahm et al. 2004, Qiu et al. 2005]. Recientemente, Raju y coautores [Raju et al. 2009] estudiaron las propiedades estructurales, eléctricas y ópticas de películas de óxido de plata crecidas a varias presiones de oxígeno. Los cambios en estas propiedades dependen de los tamaños de grano de $\mathrm{Ag}_{2} \mathrm{O}$ que, a su vez, están relacionadas al proceso de deposición. Cuando el óxido de plata es crecido sobre una delgada película de plata mediante la exposición de una superficie metálica libre en un flujo de $\mathrm{O}_{2}$ [Gao et al. 2004, Sahm et al. 2004, Qiu et al. 2005] o en un plasma rico en oxígeno [Libardi and Grieneisen 1998], el espesor del óxido de plata aumenta a expensas de una disminución del espesor de la plata. El proceso de oxidación progresivo produce cambios notables en las propiedades de toda la estructura plata-óxido de plata. Para aplicaciones donde la oxidación es una consecuencia directa de la presencia de oxígeno libre en medios líquidos o gaseosos, o cuando cierto espesor de óxido debe ser 
obtenido para un uso específico, es importante tener una medición directa del espesor de la capa de óxido de plata durante el proceso de crecimiento. Tradicionalmente, esto se ha llevado a cabo utilizando transmitividad de luz blanca, basado en la transparencia de la capa de $\mathrm{Ag}_{2} \mathrm{O}$ en la región visible [Gao et al. 2004, Qiu et al. 2005], o con elipsometría espectroscópica [Pettersson and Snyder 1995], que permite determinar el espesor final y las constantes ópticas.

En esta Tesis se analizaron las características de las respuestas plasmónicas de películas metálicas delgadas de plata depositadas sobre sustratos dieléctricos transparentes para desarrollar una técnica precisa y de bajo costo para una medición confiable y directa del espesor in situ.

La resonancia del polaritón plasmón superficial (SPPR, Surface Plasmon Polariton Resonance) es una técnica versátil capaz de monitorear procesos químicos, físicos y biológicos en las inmediaciones de interfaces metálicas [de Brujin et al. 1991, Rahn and Hallock 1995, Homola et al. 1999]. La excitación óptica de SPPR en una película metálica, cuando una luz monocromática de onda polarizada $p$ alcanza la interfase vidrio-metal a un cierto ángulo mayor que el ángulo de reflexión interna total (TIR, Total Internal Reflection), depende de una combinación del dieléctrico, del metal y del medio que la rodea. Una de las estructuras más simples en la que se puede obtener esta excitación es la configuración de Kretschmann, descripta en la Sección 4.3.

Aunque el haz de onda polarizada $s$ no puede excitar el SPP [Novotny and Hecht 2007], se puede utilizar para lograr modos propagantes en guías de onda plana, en lo que se conoce como acoplamiento del modo de guía de onda óptica (OWMC, Optical Waveguide Mode Coupling). Cuando se excita un modo en la guía de onda por una onda incidente oblicua, parte de la energía se extrae de ella, por lo que la onda reflejada en la interfase tendrá un mínimo en la intensidad para un dado ángulo de incidencia. 
Los sensores tipo Kretschmann son conocidos como dispositivos de sensado de multicapas nanométricas. En este sentido, la investigación de esta Tesis en esta área estuvo centrada en dos objetivos: en primer lugar, el diseño de un sensor de oxígeno basado en un dispositivo Kretschmann a partir de la determinación del espesor de $\mathrm{Ag}_{2} \mathrm{O}$ y, en segundo lugar, el desarrollo de un protocolo de medidas para determinar el espesor de óxido de plata, cuando una película de plata de espesor inicial conocido se convierte gradualmente en óxido de plata por la exposición a una atmósfera controlada rica en oxígeno.

Las características particulares de las curvas de reflectividad de la estructura de multicapas $\left(\mathrm{Ag}-\mathrm{Ag}_{2} \mathrm{O}\right)$ fueron estudiadas tanto para polarización de onda $p$ como para $s$ en función del ángulo de incidencia y del espesor de la capa.

\subsubsection{Diseño de un sensor de oxígeno basado en un dispositivo Kretschmann}

En la Figura 7.8 (a) se muestra un posible arreglo experimental típico para sensar la variación del espesor de una capa de $\mathrm{Ag}_{2} \mathrm{O}$ de unas decenas de nanómetros por encima de una película de plata usando las técnicas de SPPR y OWMC, cuando el índice de refracción complejo de plata y óxido de plata son conocidos. Un haz láser de He-Ne incide sobre un sensor tipo Kretschmann montado en un goniómetro después de pasar a través de un modulador mecánico (chopper), que le imprime una frecuencia de referencia que se aplica a un amplificador lock-in (LIA, Lock-In Amplifier). La señal reflejada es detectada por un fotodetector (fotomultiplicador o fotodiodo) cuya salida alimenta la entrada de la señal del LIA. La salida del LIA entra al canal A del divisor de señales A/B. Una fracción del haz incidente se muestrea con un divisor de haz y un fotodiodo que a su vez alimenta el canal 
B del divisor de señales para tener en cuenta las posibles fluctuaciones lentas en la intensidad del láser. Una PC controla el goniómetro y registra la señal de CC que proviene del divisor de señales. En la Figura 7.8 (b) se representa en detalle la posible configuración tipo Kretschmann diseñada específicamente para detectar la formación de la capa de óxido de plata de espesor $t$ crecida sobre una película de plata delgada de espesor $d$ que se encuentra depositada sobre un prisma cuyo material es, por ejemplo, BK7. La multicapa $\mathrm{Ag}-\mathrm{Ag}_{2} \mathrm{O}$ puede estar contenida en una cámara de reacción donde fluye una mezcla de oxígeno y un gas inerte para varias concentraciones relativas y velocidades de oxígeno.

Se ha utilizado un prisma cuyo material es BK7 como parte del sensor de configuración tipo Kretschmann. Cuando se produce la oxidación aplicando el procedimiento estándar de circulación de oxígeno sobre la muestra [Libardi and Grieneisen 1998, Gao et al. 2004, Qiu et al. 2005], el espesor del óxido de plata $t$ crece a expensas de una disminución del espesor de la capa de plata $d$.

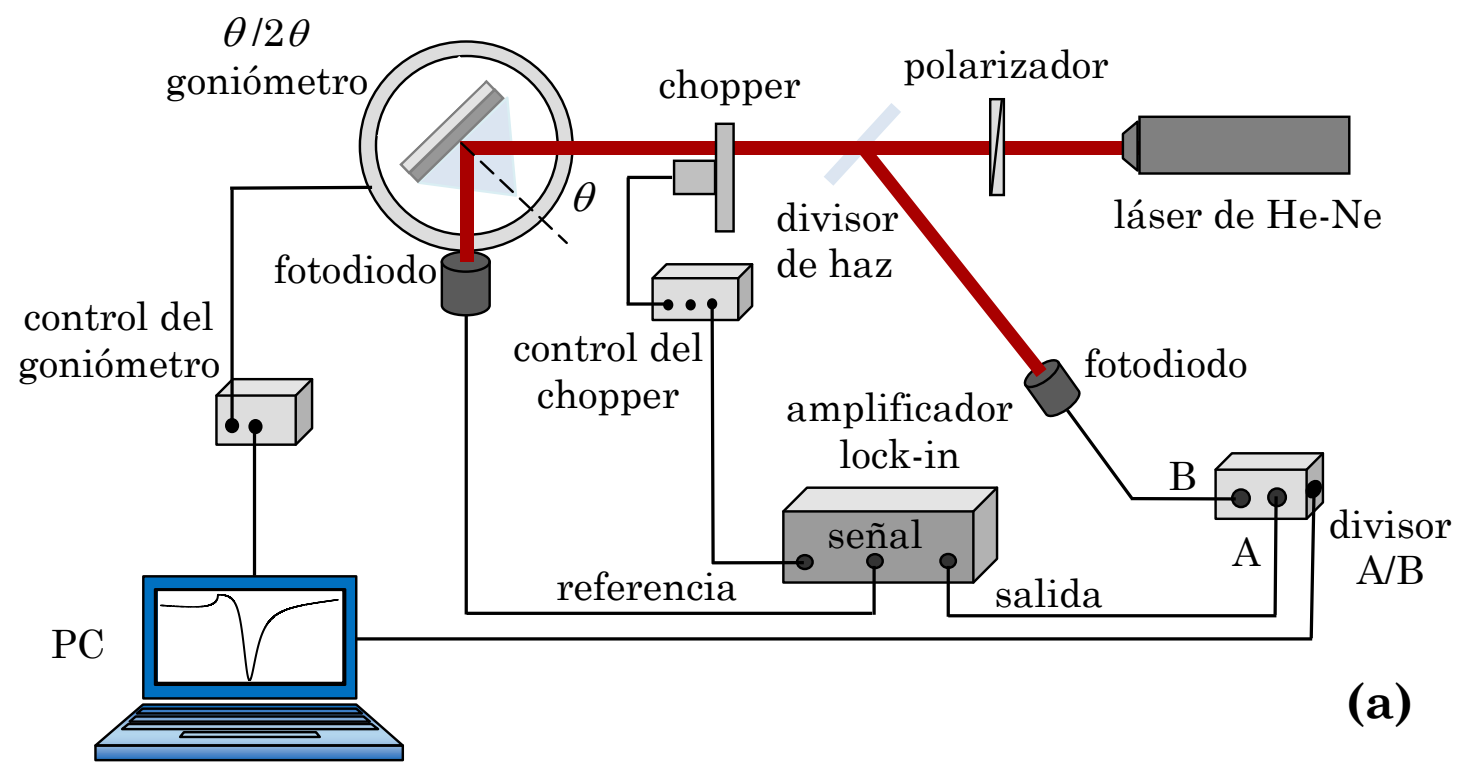




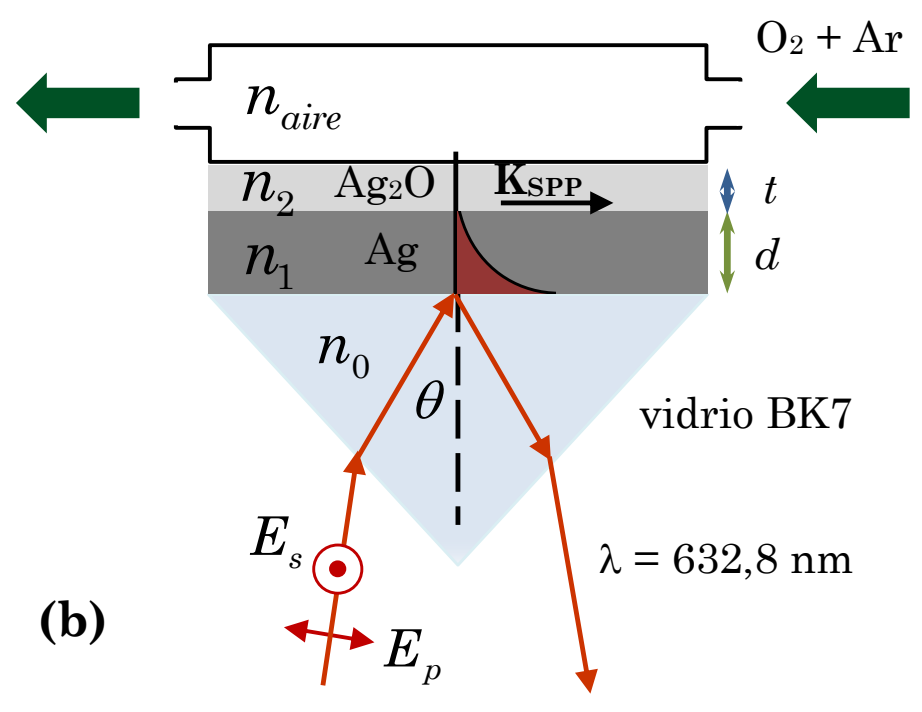

Figura 7.8: (a) Arreglo experimental propuesto para sensar el crecimiento del espesor del óxido de plata sobre una película de plata usando SPPR y OWMC; (b) detalle de la configuración de Kretschmann.

El par de espesores $t$ - $d$ que optimiza el ajuste de los datos experimentales tomados de Libardi y Grieneisen [Libardi and Grieneisen 1998] están representados con puntos en la Figura 7.9, junto con un ajuste correspondiente a una regresión lineal. El módulo de la pendiente de esta regresión es 0,67, arrojando un valor final para el espesor de $\mathrm{Ag}_{2} \mathrm{O}$ (cuando no existen restos de Ag) cerca de 1,5 veces más grande que el espesor inicial de $\mathrm{Ag}$, de acuerdo con los resultados obtenidos por Schmidt y coautores [Schmidt et al. 1996].

Dado que el mecanismo de conversión de plata en óxido de plata al exponer el metal a una atmósfera de oxígeno está gobernado por la reacción:

$$
4 \mathrm{Ag}+\mathrm{O}_{2} \rightarrow 2 \mathrm{Ag}_{2} \mathrm{O}
$$

la relación lineal de la Figura 7.9 se puede utilizar para calibrar la cantidad de $\mathrm{O}_{2}$ a partir de la determinación del espesor de $\mathrm{Ag}_{2} \mathrm{O}$. Un valor inicial de 
$45 \mathrm{~nm}$ para el espesor de la capa de plata permitió determinar el espesor del óxido de plata en el rango de 0 a $67 \mathrm{~nm}$ durante el proceso de oxidación. Las técnicas de crecimiento de películas de óxido de plata permiten un control preciso del espesor del metal del orden de $1 \mathrm{~nm}$ o menos [Libardi and Grieneisen 1998].

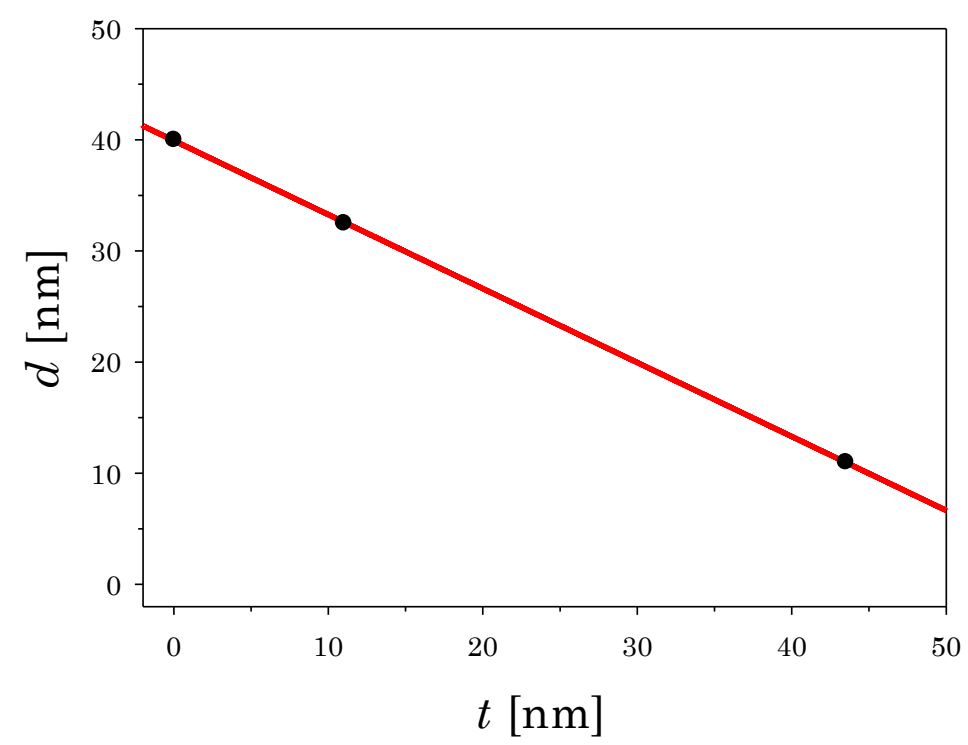

Figura 7.9: Relación entre el espesor de plata $(d)$ y el espesor del óxido de plata $(t)$. Los círculos representan los datos tomados de [Libardi and Grieneisen 1998]. La línea sólida es un ajuste lineal de estos datos.

Para sensar la tasa de crecimiento de la película de óxido de plata $\mathrm{Ag}_{2} \mathrm{O}$, se han analizado diferentes parámetros característicos de las curvas de reflectividad del sistema multicapa de plata-óxido de plata tanto para polarización de onda $p$ (a través del estudio SPPR) como $s$ (a través de OWMC).

El formalismo utilizado para analizar el comportamiento de la reflectividad está relacionando a la matriz característica de un sistema de multicapas para ambas polarizaciones, como se ha descripto en la Sección 
4.2. Para este caso especial, el sistema está formado por dos capas (plata y óxido de plata) depositadas sobre un prisma de vidrio, siendo el vidrio y el aire medios semi-infinitos, como se puede ver en la Figura 7.8 (b).

\subsubsection{Análisis de reflectividad para polarizaciones $p$ y $s$}

Las curvas de SPPR y OWMC en función del ángulo de incidencia han sido calculadas de acuerdo con el formalismo de las ecuaciones (4.18) a (4.22) de la Sección 4.2.

La Figura 7.10 muestra las curvas de reflectancia para polarización de onda $p$ correspondientes a diferentes espesores de óxido de plata, teniendo en cuenta la regresión lineal de la Figura 7.9. Los datos utilizados para los cálculos fueron: $\lambda=632,8 \mathrm{~nm}$, el índice de refracción del BK7 a esta longitud de onda es $n_{0}=1,515$ [Schott]. Los valores experimentales del índice de refracción de $\mathrm{Ag}\left(n_{1}\right)$ y $\mathrm{Ag}_{2} \mathrm{O}\left(n_{2}\right)$ se tomaron de Johnson y Christy y Pettersson y Snyder [Pettersson and Snyder 1995], respectivamente, mientras que el índice de refracción del aire se tomó como $n_{\text {aire }}=1$.

Se puede observar que la reflexión total interna ocurre para un $\theta_{\mathrm{TIR}}=39,4^{\circ}$, según el índice de refracción del prisma de BK7 y el aire como medio circundante. La primera curva de la izquierda corresponde a la capa de plata sin oxidar de $45 \mathrm{~nm}$ de espesor, mostrando un mínimo en la reflectividad a $\theta_{0}=41,8^{\circ}$. Cuando una capa de óxido de plata comienza a crecer, se puede ver un desplazamiento del mínimo hacia ángulos más grandes. De los cálculos de reflectividad se puede notar que los pequeños espesores de óxido de plata producen un notable corrimiento del mínimo $\left(\theta^{\prime}\right)$ con respecto a $\theta_{0}$ como así también un ensanchamiento del FWHM. Además, para ángulos más pequeños que el ángulo de TIR, la reflectividad 
disminuye a medida que la capa de óxido de plata aumenta. En particular, la reflectividad ( $h$ ) a un ángulo fijo de $25^{\circ}$ que se muestra en la Figura 7.10 está relacionada a los cambios mencionados. Si el espesor de la capa de plata permaneciera constante, no se observarían cambios en la reflectividad h.

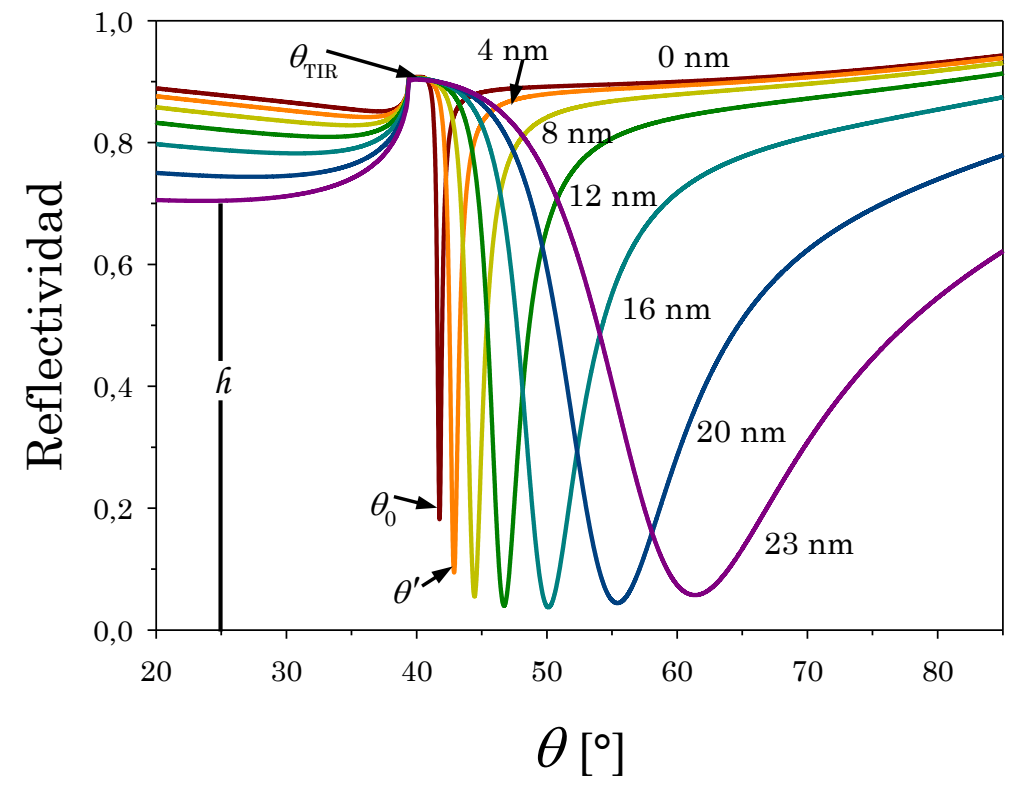

Figura 7.10: Reflectividad de onda polarizada $p$ calculada en función del ángulo de incidencia para diferentes espesores de $\mathrm{Ag}_{2} \mathrm{O}$ crecidos sobre una película de plata de $d=45 \mathrm{~nm}$. El mínimo de la reflectividad está indicado por $\theta^{\prime}$.

El ángulo del mínimo en la reflectividad $\theta^{\prime}$ tiene un desplazamiento no lineal cuando aumenta el espesor del óxido de plata $t$. La Figura 7.11 representa $\theta^{\prime}$ para ondas polarizadas $p$ en función de $t$. Los puntos calculados se pueden ajustar por una función exponencial (línea sólida) que relaciona unívocamente el ángulo correspondiente al mínimo de reflectividad en función de $t$ en un rango de 0 a $30 \mathrm{~nm}$. La incerteza teórica en la determinación de $t$ disminuye para espesores crecientes. Las barras de 
error calculadas, asumiendo que el paso del goniómetro es $0,1^{\circ}$, son del tamaño de los puntos azules o menores cuando $t$ aumenta.

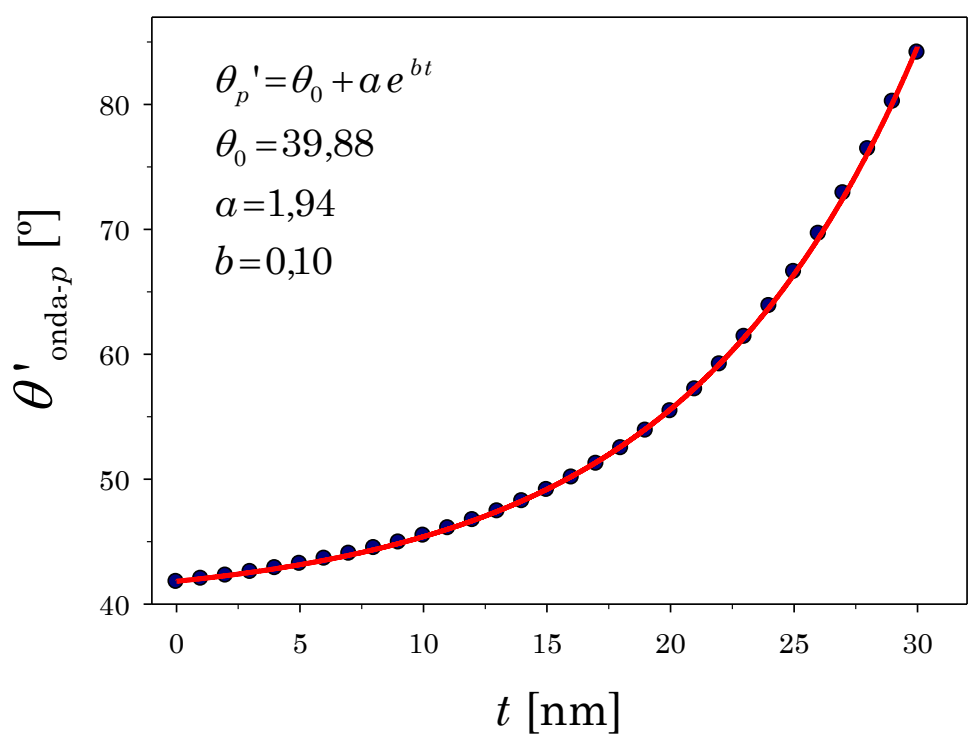

Figura 7.11: $\theta^{\prime}$ para una onda polarizada $p$ en función del espesor del óxido de plata $t$. La línea sólida representa la curva de ajuste exponencial en el rango de espesores de 0 a $30 \mathrm{~nm}$.

Observando nuevamente la Figura 7.10, se puede ver que existe una diferencia apreciable en la reflectividad cuando el espesor del óxido de plata aumenta si el ángulo de observación se mantiene fijo en $\theta_{0}=41,8^{\circ}$ (correspondiente a plata sin oxidar). La Figura 7.12 muestra el comportamiento de la reflectividad diferencial $R \equiv R\left(\theta_{0}, t\right)-R\left(\theta_{0}, 0\right)$ en función de $t$. Se puede notar que la reflectividad diferencial satura para espesores más grandes que $5 \mathrm{~nm}$. Sin embargo, es un parámetro muy sensible para espesores menores a este valor, sugiriendo que puede ser efectivamente usado para determinar espesores de monocapas de óxido en este rango. En el recuadro de la Figura 7.12 se puede ver una ampliación de la reflectividad para $0 \mathrm{~nm}<t<5 \mathrm{~nm}$ junto con la correspondiente curva de 
ajuste y las barras de error calculadas asumiendo una incerteza en la medida experimental de $R$, de $\Delta R=0,03$. La alta sensibilidad de las medidas de reflectividad en este rango resulta de la combinación de dos características: el desplazamiento notable de la resonancia del polaritón plasmón superficial para pequeños espesores del óxido junto con la agudeza del mínimo de plata, como se puede ver en la Figura 7.10.

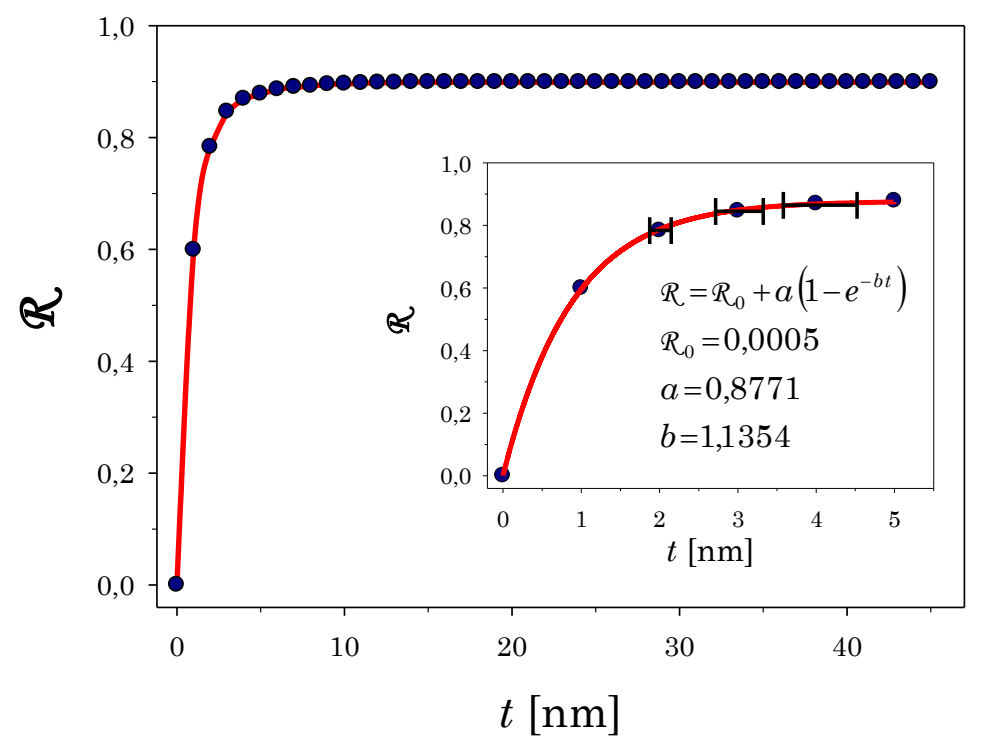

Figura 7.12: Reflectividad diferencial $R$ calculada en función del espesor del óxido de plata $t$ para una onda polarizada $p$. En el recuadro se muestra una ampliación de la curva en el rango de espesores de óxido de 0 a $5 \mathrm{~nm}$.

El otro parámetro característico de las curvas de reflectividad es el FWHM para polarización de onda $p$. Como puede verse en la Figura 7.10, el FWHM aumenta de forma monótona cuando $t$ aumenta. La Figura 7.13 muestra el FWHM de las curvas de reflectividad representadas en función del espesor del óxido de plata, junto con las barras de error para algunos valores de $t$, asumiendo una incerteza para FWHM de $0,2^{\circ}$. Este parámetro sólo puede ser definido adecuadamente para $t \leq 23 \mathrm{~nm}$, ya que para 
espesores mayores las curvas de reflectividad carecen de la simetría necesaria para definir el FWHM. El FWHM tiene una sensibilidad mayor para $t>5 \mathrm{~nm}$. Esta característica ofrece información complementaria para la reflectividad diferencial $\mathbb{R}$ que, según lo indicado arriba, muestra más sensibilidad para espesores menores que $5 \mathrm{~nm}$.

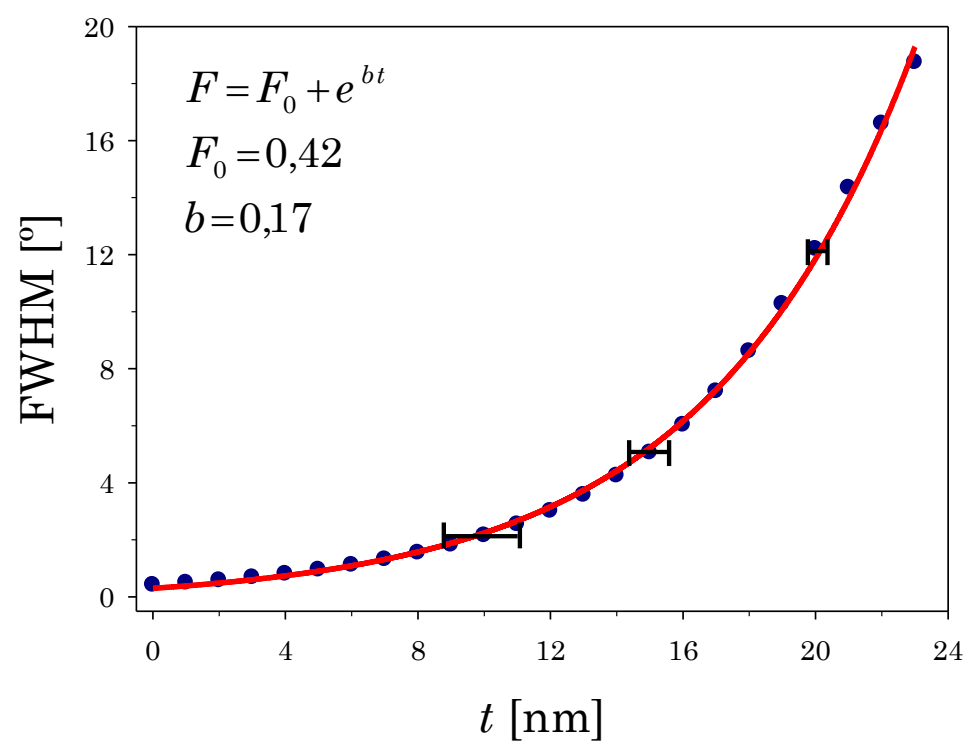

Figura 7.13: FWHM (indicado como $F$ en el recuadro de la ecuación) de la curva de reflectividad calculada para una onda polarizada $p$ para diferentes espesores del óxido de plata $t$ crecido sobre una película de plata.

Como se indica en la descripción de las curvas en la Figura 7.10, otro parámetro de interés para la caracterización de espesores del óxido de plata es la reflectividad para ángulos más pequeños que el ángulo crítico TIR ( $h$ ). Dado que $t$ aumenta a expensas de la disminución de $d$, en una relación dada por la Figura 7.9, $h$ varía con el espesor de la capa de $\mathrm{Ag}_{2} \mathrm{O}$. Por inspección de las curvas en la Figura 7.10, se puede ver que, para $\theta=25^{\circ}$ la reflectividad barre un rango apropiado de valores, que se pueden medir con 
una suficiente precisión. Esta reflectividad $h$ para $25^{\circ}$ y polarización de onda $p$ se grafica en función de $t$ en la Figura 7.14.

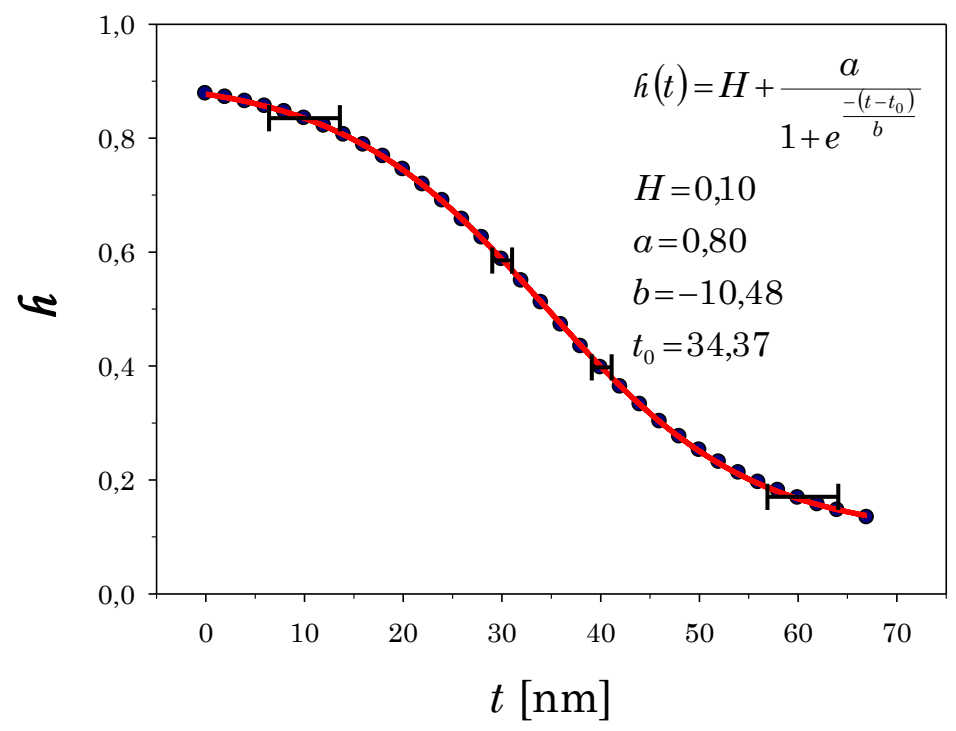

Figura 7.14: Valores calculados (círculos) y ajuste lineal (línea sólida) de la reflectividad $h$ para un ángulo de incidencia de $25^{\circ}$, para una onda polarizada $p$ en función del espesor de $\mathrm{Ag}_{2} \mathrm{O}$.

Los puntos correspondientes a los valores calculados, se pueden ajustar por una curva tipo sigmoidea. Aunque esta curva es una función monótona del espesor del óxido $t$ en el rango completo de 0 a $67 \mathrm{~nm}$, la mayor sensibilidad, está dada por la pendiente más grande, para la región central $(20 \mathrm{~nm}<t<45 \mathrm{~nm})$. Para aclarar este punto, las barras de error para ciertos valores de $t$ son calculadas asumiendo una incerteza en $h$ de 0,03 .

Para espesores de $\mathrm{Ag}_{2} \mathrm{O}$ más grande que $45 \mathrm{~nm}$, el análisis para ondas con polarización $p$ falla, ya que el ángulo del mínimo del plasmón $\left(\theta^{\prime}\right)$ cae más allá del rango medible $\left(90^{\circ}\right)$, el FWHM no puede definirse, la reflectividad diferencial $R$ tiene un valor constante independiente del 
espesor y $h$ tiene muy poca sensibilidad. En este caso, es interesante explorar la reflectividad para un haz de polarización de onda $s$ en la configuración de Kretschmann.

La Figura 7.15 muestra la reflectividad en función del ángulo de incidencia para varios espesores de $\mathrm{Ag}_{2} \mathrm{O}$. Para el rango de $42 \mathrm{~nm}<t<55 \mathrm{~nm}$ (Figura 7.15 (a)), se puede observar que, a medida que el espesor del óxido de plata aumenta, los mínimos se hacen más amplios y se desplazan hacia ángulos mayores más allá del ángulo de TIR. Este mínimo se debe al acoplamiento de un modo guiado en la capa de óxido, que actúa como una guía de onda óptica con pérdidas. El modo guiado acoplado toma energía del haz incidente, generando así un mínimo en las curvas de reflectancia. La posición angular del mínimo $\theta^{\prime}$ es una función monótonamente creciente de $t$, aunque más allá de $55 \mathrm{~nm} \theta^{\prime}$ cae fuera del rango de ángulos medibles.

La Figura 7.15 (b) representa las curvas de reflectividad en el rango de $56 \mathrm{~nm}<t<67 \mathrm{~nm}$. En este último caso, no se observan mínimos y decae con pendiente negativa a partir del ángulo TIR.

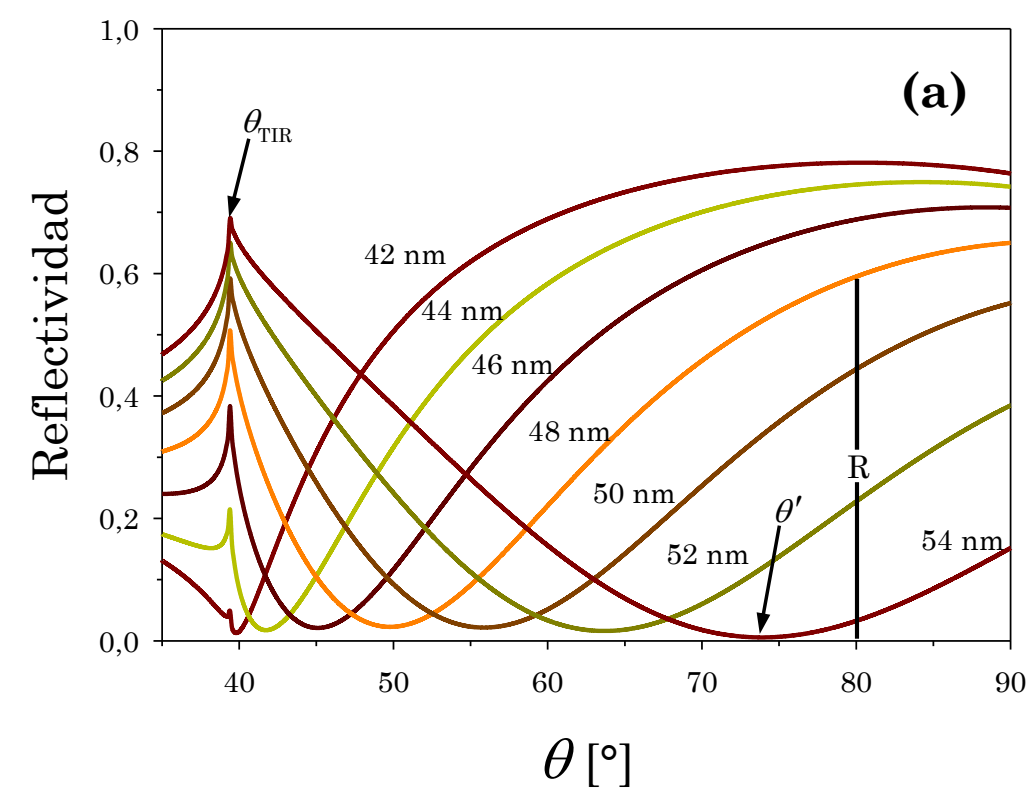




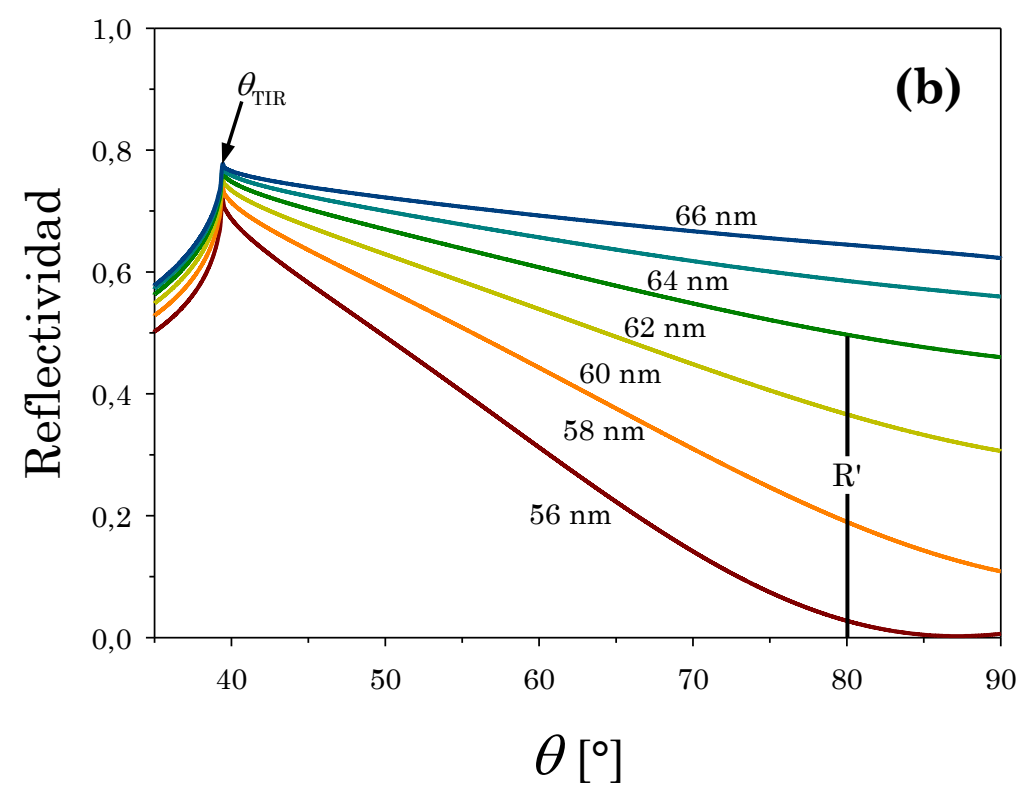

Figura 7.15: (a) Curvas de reflectividad para una onda polarizada $s$ en función del ángulo de incidencia para diferentes espesores de $\mathrm{Ag}_{2} \mathrm{O}$ en el rango de 42 a $55 \mathrm{~nm}$; (b) curvas de reflectividad para una onda polarizada $s$ con pendiente negativa para diferentes espesores de $\mathrm{Ag}_{2} \mathrm{O}$ en el rango de 56 a $67 \mathrm{~nm}$.

Teniendo en cuenta las consideraciones anteriores, la Figura 7.16 grafica $\theta^{\prime}$ en función de $t$ en el rango de $42 \mathrm{~nm}<t<55 \mathrm{~nm}$.

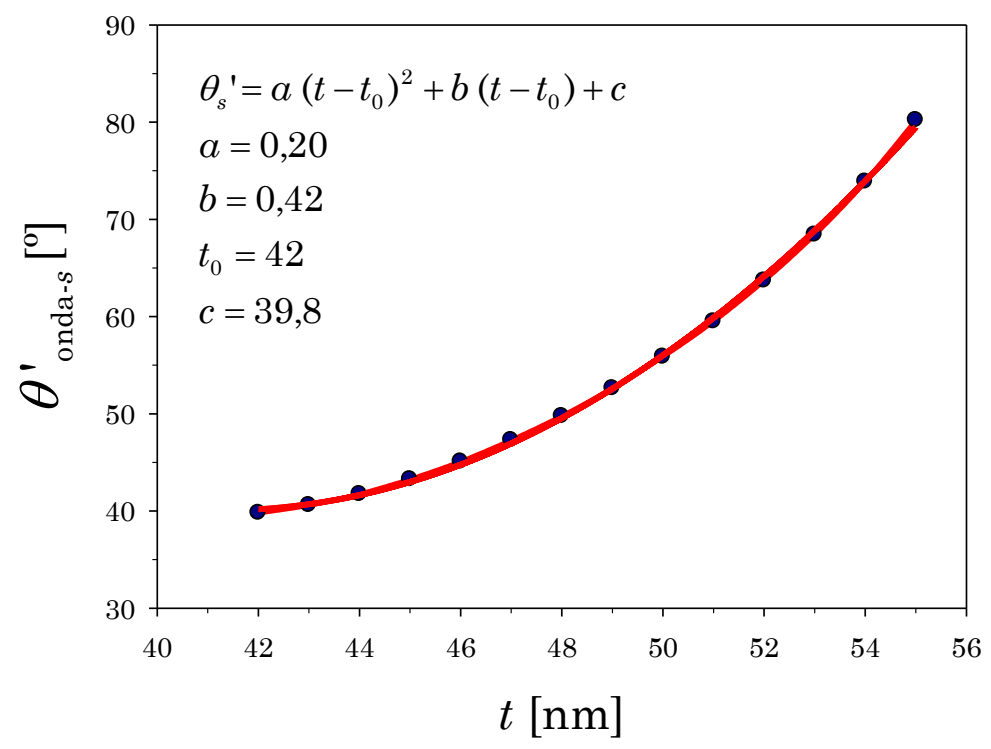

Figura 7.16: Mínimo de la reflectividad ( $\theta^{\prime}$ ) en función de espesores de $\mathrm{Ag}_{2} \mathrm{O}$ para una onda polarizada $s$. Los círculos corresponden a los valores calculados y la línea sólida a un ajuste cuadrático. 
En este caso, los puntos calculados se pueden ajustar por una curva cuadrática con los coeficientes que figuran en el recuadro, siendo $t_{0}$ el valor del espesor inicial para este rango y $c$ el ángulo mínimo de la reflectividad. La incerteza en la determinación de espesores va desde 0,25 a $0,02 \mathrm{~nm}$ (nominal), cuando $t$ se extiende de 42 a $55 \mathrm{~nm}$, respectivamente. En este rango de espesores, un parámetro alternativo para la determinación de $t$ es la reflectividad en un ángulo fijo más allá del ángulo de TIR. Como se puede observar en la Figura 7.15, para un ángulo de $80^{\circ}$, las curvas de reflectividad cubren un amplio rango de valores.

La Figura 7.17 muestra valores de reflectividad calculados a $80^{\circ}$ para polarización de onda $s$ en función de $t$ en el rango de $42 \mathrm{~nm}<t<55 \mathrm{~nm}$.

Se puede observar que, en el rango $56 \mathrm{~nm}<t<67 \mathrm{~nm}$, las curvas de reflectividad tienen una pendiente negativa, mientras que las del rango de $42 \mathrm{~nm}<t<55 \mathrm{~nm}$ tienen una pendiente positiva en $\theta=80^{\circ}$.

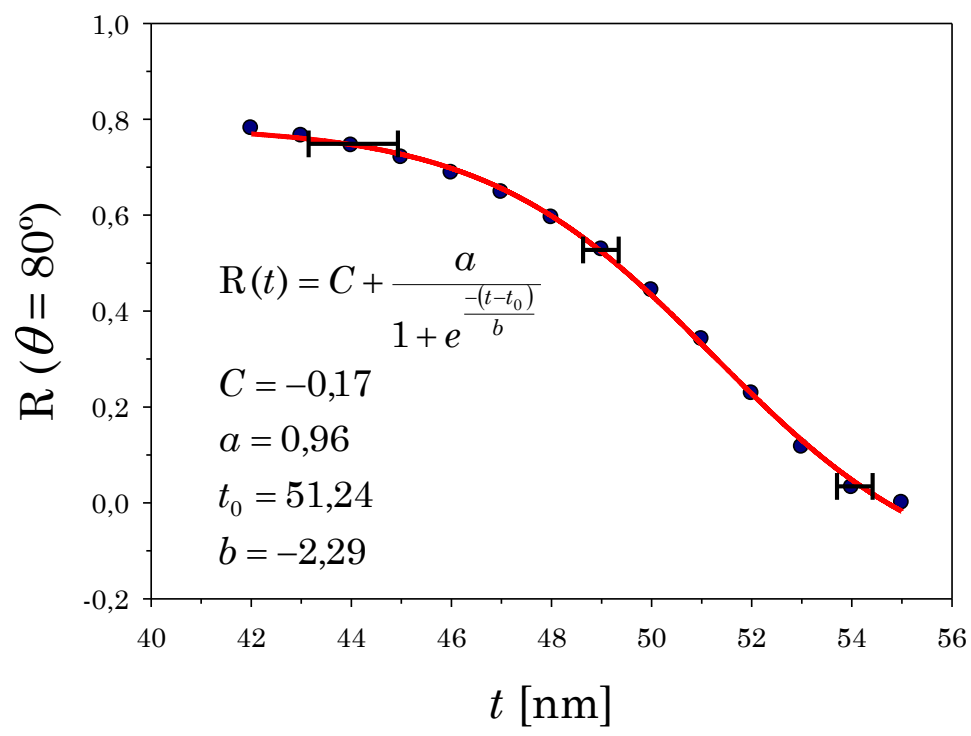

Figura 7.17: Reflectividad para $\theta=80^{\circ}$ en función de espesores de $\mathrm{Ag}_{2} \mathrm{O}$ para una onda polarizada $s$. Las barras de error se calcularon considerando una incerteza en la medida de la reflectividad de 0,03 . 
La Figura 7.18 representa los valores de las curvas de reflectividad R' con pendiente negativa en $80^{\circ}$ en función de $t$, junto con un ajuste tipo sigmoidea.

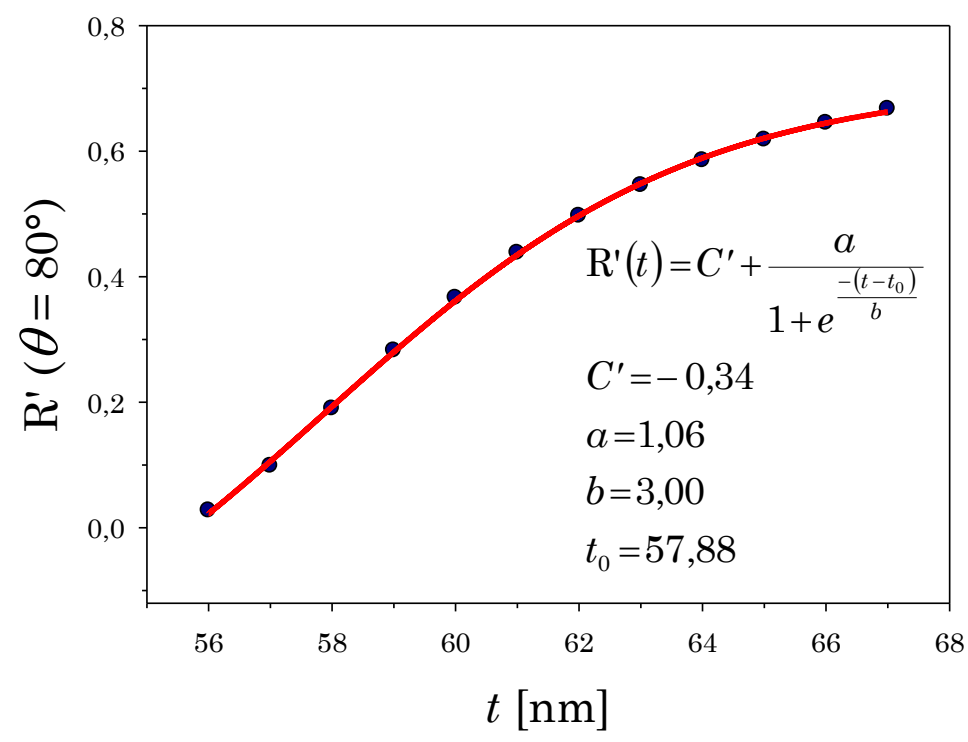

Figura 7.18: Reflectividad $R^{\prime}$ (con pendiente negativa) para $\theta=80^{\circ}$ en función de espesores de $\mathrm{Ag}_{2} \mathrm{O}$ para una onda polarizada $s$.

La medida experimental de la reflectividad a $80^{\circ}$ y su pendiente se pueden utilizar para calcular el espesor de óxido $t$ en el intervalo de $56 \mathrm{~nm}<t<67 \mathrm{~nm}$.

\subsubsection{Procedimiento híbrido de reflectancia SPPR- OWMC para la determinación de espesores de $\mathrm{Ag}_{2} \mathrm{O}$}

Teniendo en cuenta las características de las curvas de reflectividad tanto para ondas de polarización $p$ como $s$ estudiadas en la sección anterior, se puede establecer un procedimiento híbrido de reflectancia SPPR-OWMC 
para medir espesores de óxido, siguiendo el arreglo esquemático de la configuración de la Figura 7.8:

1) Se configura el prisma de Krestshmann con una película de plata inicial de $45 \mathrm{~nm}$ en un goniómetro con una precisión de al menos $0,1^{\circ}$.

2) Antes del crecimiento de cualquier óxido de plata, se ajusta el polarizador a un estado de onda $p$ y se determina la posición angular del mínimo del plasmón $\left(41,8^{\circ}\right)$ para la película inicial de plata.

3) Cuando comienza el proceso de oxidación, se mide la reflectividad diferencial $R$ a un ángulo fijo de $41,8^{\circ}$. De la ecuación del recuadro de la Figura $7.12, R=5 \times 10^{-4}+0,88\left(1-e^{-1,13 t}\right)$, se puede determinar el espesor del óxido en el rango de $0 \mathrm{~nm}<t<5 \mathrm{~nm}$. Alternativamente, se puede utilizar la medida de la posición angular del mínimo de la reflectividad $\theta_{p}{ }^{\prime}=39,88+1,94 e^{0,1 t}$ aunque con menor precisión.

4) Cuando la reflectividad diferencial satura, se mide el mínimo $\theta^{\prime}$ para una onda polarizada $p$ y se determina el espesor $t$ para el intervalo de 5 a $30 \mathrm{~nm}$ usando la ecuación $\theta_{p}{ }^{\prime}=39,88+1,94 e^{0,1 t}$ (Figura 7.11). Alternativamente en el rango de 5 a $25 \mathrm{~nm}$, se puede utilizar el análisis del FWHM, aunque los ángulos deben ser completamente explorados en el rango de $0^{\circ}$ a $90^{\circ}$ para calcular FWHM, en este caso se debe utilizar la ecuación $F=0,42 e^{0,17 t}$.

5) En el rango de $20 \mathrm{~nm}<t<45 \mathrm{~nm}$, es posible evaluar la reflectividad para una onda polarizada $p$ a $25^{\circ}$ ( $h$ en la Figura 
7.14) y determinar el espesor del óxido de plata a través de la ecuación $h=0,1+0,8 /\left[1+e^{(t-34,37) / 10,48}\right]$.

6) Para el rango de $42 \mathrm{~nm}<t<55 \mathrm{~nm}$, se cambia el polarizador a la posición de polarización de onda $s$, se mide el mínimo de reflectividad $\theta^{\prime}$ y determina el espesor del óxido de plata a través de la ecuación $\theta_{s}{ }^{\prime}=0,2(t-42)^{2}+0,42(t-42)+39,8$ (Figura 7.16). Alternativamente, se puede medir la reflectividad $\mathrm{R}$ a $80^{\circ}$ con pendiente positiva y obtener el valor del espesor del óxido usando $\mathrm{R}(t)=-0,17+0,96 /\left[1+e^{(t-51,24) / 2,29}\right]$ (Figura 7.17).

7) Finalmente, para medir el intervalo de $56 \mathrm{~nm}<t<67 \mathrm{~nm}$, es posible evaluar la reflectividad $R^{\prime}$ a $80^{\circ}$ para polarización de onda $s$ con pendiente negativa. Los espesores se pueden determinar utilizando la ecuación $\mathrm{R}^{\prime}(t)=-0,34+1,06 /\left[1+e^{-(t-57,88) / 3}\right]$ (Figura 7.18).

La Tabla 7.1 resume los parámetros apropiados medibles usados para determinar el espesor del óxido en cada rango, junto con las ecuaciones de ajuste que relacionan los diferentes parámetros con el espesor $t$. Es importante señalar que la interrogación angular completa de polarización de onda $p$ y el ajuste completo de la curva de reflectividad es el método tradicional en la configuración de Kretschmann. En este trabajo de Tesis se muestra un camino alternativo para usar características específicas de las curvas de onda $p$ y $s$ a ciertos ángulos fijos sin la necesidad de la interrogación angular completa, lo que permite determinar el espesor de $\mathrm{Ag}_{2} \mathrm{O}$ durante su crecimiento por la exposición de una película de $\mathrm{Ag}$ a un flujo de oxígeno. Basado en este enfoque, es posible determinar espesores de $\mathrm{Ag}_{2} \mathrm{O}$ en el rango de 0 a $45 \mathrm{~nm}$ utilizando la medición de reflectividad para 
polarización de onda $p$. Del mismo modo, la reflectividad de onda $s$ en un determinado ángulo permite extender el rango de espesores hasta $70 \mathrm{~nm}$.

Tabla 7.1: Parámetros medibles para determinar espesores de $\mathrm{Ag}_{2} \mathrm{O}$

\begin{tabular}{|c|c|c|}
\hline $\begin{array}{c}\text { Espesor de } \mathrm{Ag}_{2} \mathrm{O} \\
t[\mathrm{~nm}]\end{array}$ & Parámetros m & dibles \\
\hline 0 a 5 & $\begin{array}{c}\mathcal{R}(\text { onda } p) \\
R=5 \times 10^{-4}+0,88\left(1-e^{-1,13 t}\right)\end{array}$ & $\begin{array}{c}\theta_{\min }(\text { onda } p) \\
\theta_{p}{ }^{\prime}=39,88+1,94 e^{0,1 t}\end{array}$ \\
\hline 5 a 30 & $\theta_{\min }($ onda $p) \quad \theta_{p}{ }^{\prime}=$ & $9,88+1,94 e^{0,1 t}$ \\
\hline 0 a 25 & FWHM (onda $p$ ) $I$ & $=0,42 e^{0,17 t}$ \\
\hline 20 a 45 & $h\left(\right.$ a $25^{\circ}$, onda $\left.p\right) \quad h=0,1+$ & $0,8 /\left[1+e^{(t-34,37) / 10,48}\right]$ \\
\hline \multirow{2}{*}{42 a 55} & \multicolumn{2}{|c|}{$\theta_{\min }($ onda $s)$} \\
\hline & $\begin{array}{l}\mathrm{R} \text { (a } 80^{\circ} \text {, onda } s, \text { pend } \\
\mathrm{R}(t)=-0,17+0,96\end{array}$ & $\begin{array}{l}\text { ente positiva) } \\
\left.1+e^{(t-51,24) / 2,29}\right]\end{array}$ \\
\hline 55 a 67 & $\begin{array}{l}\mathrm{R}^{\prime} \text { (a } 80^{\circ} \text {, onda } s \text {, pend } \\
\mathrm{R}^{\prime}(t)=-0,34+1,06\end{array}$ & $\begin{array}{l}\text { ente negativa) } \\
{\left[1+e^{-(t-57,88) / 3}\right]}\end{array}$ \\
\hline
\end{tabular}




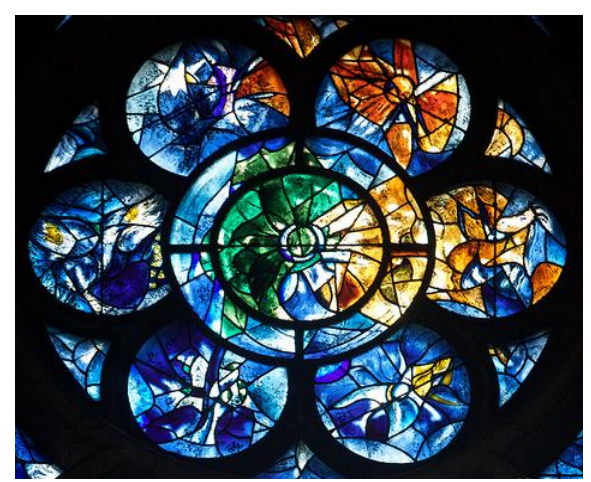

Catedral de Ntra. Sra. de Reims Marne, Francia

\section{Capítulo 8}

\section{Conclusiones}

La plasmónica explora las propiedades y características de las resonancias ópticas que presentan estructuras nanométricas empleadas en diferentes campos que son del dominio de la ingeniería y de la física aplicada. En este contexto esta Tesis ha estudiado las resonancias plasmónicas de dos tipos de estructuras: nanoesferas con y sin recubrimiento por un lado, y películas delgadas de espesor nanométrico por el otro, desde el punto de vista de sus propiedades ópticas en el campo lejano.

Se ha combinado un aspecto de desarrollo experimental y otro relacionado con el modelado teórico de las propiedades ópticas de nanoestructuras esféricas simples y núcleo-recubrimiento con el fin de complementar ambos, reforzando y enriqueciendo la interpretación de los resultados obtenidos. Por un lado, el desarrollo experimental estuvo relacionado con la generación de partículas nanométricas y subnanométricas por ablación láser de blancos sólidos de cobre y plata con pulsos ultracortos. Asimismo se obtuvieron espectros de extinción óptica y Raman a partir de las suspensiones coloidales generadas. Por otro lado, el aspecto teórico abarcó el modelado de la función dieléctrica compleja para 
partículas de diferentes radios nanométricos y subnanométricos para su utilización en el cálculo de los correspondientes coeficientes de extinción, ambos en función de la longitud de onda.

Todos estos estudios generales dieron lugar a avances tanto en aspectos básicos como aplicados.

Como conclusiones generales, luego del análisis de las propiedades ópticas de nanoestructuras metálicas de cobre y plata, esta Tesis ha permitido el desarrollo original de los siguientes aspectos:

- En primer lugar, se ha modelado la función dieléctrica compleja para cobre y plata, realizando la corrección por tamaño en las contribuciones de los electrones libres y ligados, logrando obtener una expresión de la función dieléctrica total dependiente del tamaño para partículas nanométricas y subnanométricas. La modificación realizada a la contribución de los electrones libres, en ambas partes real e imaginaria de la función dieléctrica, mostró para el cobre y la plata, una mayor sensibilidad para $a<2 \mathrm{~nm}$ y un comportamiento casi coincidente con la curva correspondiente a bulk para $a=10 \mathrm{~nm}$ en la parte real. Por otra parte, la componente imaginaria mostró una tendencia límite similar al bulk para $a>20 \mathrm{~nm}$, exhibiendo gran sensibilidad para valores de radios en el rango $0,7 \mathrm{~nm}<a<10 \mathrm{~nm}$ y presentando un comportamiento límite inferior para valores que están por debajo de $0,6 \mathrm{~nm}$.

La contribución de los electrones ligados en las transiciones desde la banda $d$ hasta la banda de conducción fue modelada usando una expresión que tiene en cuenta todas las posibles transiciones interbandas. Para partículas subnanométricas, se incluyó en la función dieléctrica de los electrones ligados un factor dependiente del 
tamaño que representa el mayor espaciamiento entre los niveles electrónicos. Esta corrección, en ambas partes real e imaginaria, es importante para tamaños subnanométricos con un radio menor a $2 \mathrm{~nm}$, por encima del cual la corrección se hace despreciable tanto para el cobre como para la plata.

- A través del ajuste de los valores experimentales tomados de Johnson y Christy y utilizando el modelo de la función dieléctrica antes mencionado, se determinaron los parámetros correspondientes a la contribución de los electrones ligados de la función dieléctrica para cobre y plata macroscópicos. Estos valores, expresados en las Tablas $6.1 \quad$ y $6.2, \quad$ son: $\quad K_{b u l k}=2 \times 10^{24}, \quad E_{g}=1,95 \mathrm{eV}, \quad E_{F}=2,15 \mathrm{eV} \quad$ y $\gamma_{\text {ligados }}=1,15 \times 10^{14} \mathrm{~Hz}$ para el cobre, y $K_{\text {bulk }}=2 \times 10^{24}, E_{g}=1,91 \mathrm{eV}$, $E_{F}=4,12 \mathrm{eV}$ y $\gamma_{\text {ligados }}=1,5 \times 10^{14} \mathrm{~Hz}$ para la plata.

- Uno de los aspectos experimentales estuvo centrado en la fabricación de partículas nanométricas y subnanométricas metálicas y de óxido metálico de diferentes estructuras, a través de ablación con láser de pulsos ultracortos sobre discos metálicos de cobre y plata en distintos líquidos y a diferentes energías. Para el caso del cobre, a través de espectroscopía Raman y en base al análisis de los mecanismos de formación de los distintos tipos de óxidos, se encontró un umbral de energía (dependiente del medio líquido) que separaba diferentes composiciones de las suspensiones coloidales: una con mayor predominancia en la formación de una configuración $\mathrm{Cu}-\mathrm{Cu}_{2} \mathrm{O}$ y otra en la que mayormente predominaba la configuración $\mathrm{Cu}_{2} \mathrm{O}-\mathrm{Cu}$. El umbral de $150 \mu \mathrm{J}$ marca el valor de la energía por debajo del cual coexisten ambas especies para el caso del agua, mientras que para el caso de acetona el valor umbral corresponde a $70 \mu \mathrm{J}$. 
- A partir de la teoría de Mie se analizó teóricamente el comportamiento del coeficiente de extinción en función de la longitud de onda de estructuras simples y núcleo-recubrimiento nanométricas y subnanométricas de cobre y plata para diferentes radios de núcleo y espesores del recubrimiento.

- Se aplicó el modelado de la función dieléctrica compleja dependiente del tamaño en la expresión del coeficiente de extinción para caracterizar las estructuras simples y núcleo-recubrimiento de diferentes especies, tipos de configuración y distribución de tamaños de núcleo y espesores del recubrimiento de partículas presentes en las suspensiones coloidales generadas, a partir del ajuste de los espectros de extinción experimentales, mediante teoría de Mie. La técnica de espectroscopía de extinción óptica demuestra ser una técnica muy eficaz, confiable y con una muy buena estadística de muestreo. Los resultados obtenidos en esta Tesis fueron comparados con los obtenidos por microscopía HRTEM y AFM.

- Para el diseño de un sensor plasmónico de oxígeno, a partir de nanopartículas aisladas de plata-óxido de plata, se analizaron teóricamente el comportamiento de ciertos parámetros plasmónicos característicos de los espectros de extinción como el pico del plasmón, el FWHM y el contraste en función del radio del núcleo y espesor del recubrimiento.

En base a los estudios anteriores se estableció un procedimiento secuencial rápido y fácil, para medir el espesor de $\mathrm{Ag}_{2} \mathrm{O}$ crecido alrededor de una nanopartícula de Ag y consecuentemente determinar la presencia de oxígeno. La importancia de este método se encuentra en la posibilidad de monitorear el crecimiento de la capa de 
óxido de plata alrededor de pequeñas nanopartículas esféricas de plata en tiempo real.

- La resonancia del polaritón plasmón superficial permitió analizar y caracterizar propiedades ópticas de películas metálicas ultradelgadas. Se estudió teóricamente el comportamiento de la reflectancia en función del ángulo de incidencia y del estado de polarización $p$ y $s$ de una onda de luz monocromática incidente en un sistema multicapas tipo Kretschmann de plata-óxido de plata. Además se analizó el comportamiento de parámetros como el mínimo de la reflectancia, el ancho total a altura media y la reflectancia a determinados ángulos para ambas polarizaciones, que se ajustaron por funciones continuas apropiadas definidas para rangos específicos de espesores.

- El conocimiento de estas funciones continuas para cada uno de estos rangos permitió establecer un protocolo de medición de reflectancia híbrido de Resonancia del Polaritón Plasmón Superficial Acoplamiento del Modo de Guía de Onda Óptica para determinar y controlar in situ el espesor de la capa de óxido durante el proceso de crecimiento. También se pudo diseñar un sensor de oxígeno a través de la determinación del espesor de una capa de óxido de plata crecida sobre una lámina de plata bajo la presencia de oxígeno.

\section{Tareas futuras}

Se prevé continuar y profundizar las líneas de investigación llevadas a cabo hasta el momento e incorporar nuevas tareas. A continuación se mencionan los objetivos específicos planteados para el desarrollo de la beca postdoctoral solicitada: 
I. Fabricación y fragmentación de nanopartículas de metales nobles, de transición y aleaciones en estructuras simples y núcleo-recubrimiento por ablación láser ultrarrápida en diferentes medios líquidos. Estudio de los procesos involucrados.

II. Implementación de la técnica de "pump and probe" para el estudio de los procesos ultrarrápidos de formación de nanopartículas en líquidos y los procesos de relajación electrónica.

III. Estudio de la función dieléctrica de nanopartículas de diferentes metales considerando la dependencia con el tamaño de la contribución de los electrones libres y ligados. Cálculo del coeficiente de extinción para diferentes tipos de estructuras (simples y núcleo-recubrimiento) y de diferente conformación.

IV. Caracterización de tamaño de las suspensiones coloidales obtenidas por ablación láser, utilizando diferentes técnicas complementarias (espectroscopía de extinción, AFM, HRTEM y Raman).

V. Implementación de la técnica óptica de Z-scan para la determinación de la susceptibilidad no-lineal de las suspensiones coloidales obtenidas. Caracterización para diferentes combinaciones de medios líquidos y particulados.

VI. Fabricación y caracterización de partículas de óxido de hierro para la obtención de coloides de nanopartículas magnéticas. 


\section{Producido de la Tesis}

Algunos de los aspectos originales presentados en este trabajo de Tesis fueron reportados en las siguientes publicaciones y comunicaciones a congresos:

\section{Publicaciones}

1. Determination of nanometric $\mathrm{Ag}_{2} \mathrm{O}$ film thickness by surface plasmon resonance and optical waveguide mode coupling techniques

Jesica M. J. Santillán, Lucía B. Scaffardi, Daniel C. Schinca and Fabián A. Videla, J. Opt. 12 (4), 045002 (8pp), 2010.

2. Quantitative optical extinction-based parametric method for sizing a single core-shell $\mathrm{Ag}-\mathrm{Ag}_{2} \mathrm{O}$ nanoparticle

J. M. J. Santillán, L. B. Scaffardi and D. C. Schinca, J. Phys. D: Appl. Phys. 44 (10), 105104 (8pp), 2011. 
3. Plasmon Spectroscopy for Subnanometric Copper Particles: Dielectric Function and Core-Shell Sizing

J. M. J. Santillán, F. A. Videla, L. B. Scaffardi and D. C. Schinca, Plasmonics, DOI: 10.1007/s11468-012-9395-8, 2012.

4. Size dependent $\mathrm{Cu}$ dielectric function for plasmon spectroscopy. Characterization of colloidal suspension generated by fs laser ablation

J. M. J. Santillán, F. A. Videla, M. B. Fernández van Raap, D. C. Schinca and L. B. Scaffardi, J. Appl. Phys. 112 (5), 054319 (8pp), 2012.

5. Plasmonic properties and sizing of core-shell $\mathrm{Cu}-\mathrm{Cu} \mathrm{U}_{2} \mathrm{O}$ nanoparticles fabricated by femtosecond laser ablation in liquids

J. M. J. Santillán, F. A. Videla, D. C. Schinca and L. B. Scaffardi, Proc. SPIE 8457, Plasmonics: Metallic Nanostructures and Their Optical Properties X, 84572U, DOI: 10.1117/12.928670, 2012.

6. Analysis of the structure, configuration, and sizing of $\mathrm{Cu}$ and $\mathrm{Cu}$ oxide nanoparticles generated by fs laser ablation of solid target in liquids

J. M. J. Santillán, F. A. Videla, M. B. Fernández van Raap, D. C. Schinca and L. B. Scaffardi, J. Appl. Phys. 113 (13), 134305 (9pp), 2013.

7. Influence of size-corrected bound-electron contribution on nanometric silver dielectric function. Sizing through optical extinction spectroscopy

J. M. J. Santillán, F. A. Videla, M. B. Fernández van Raap, D. Muraca,

L. B. Scaffardi and D. C. Schinca, en redacción (2013). 
8. Self assembled fractal-like nanostructures formed by silver nanoparticles fabricated using ultrashort pulse laser ablation

J. M. J. Santillán, M. B. Fernández van Raap, D. Muraca, P. Mendoza Zelis, F. A. Videla, D. C. Schinca and L. B. Scaffardi, en redacción (2013).

\section{Capítulo de libro}

\section{SIZE-DEPENDENT OPTICAL PROPERTIES OF METALLIC NANOSTRUCTURES}

Lucía B. Scaffardi, Daniel C. Schinca, Marcelo Lester, Fabián A. Videla, Jesica M. J. Santillán and Ricardo M. Abraham Ekeroth

Capítulo de libro por invitación Ed. Springer: Size-Dependent Optical Properties of Metallic Nanostructures. In: Kumar, Challa S. S. R. (Ed.), UVVIS and Photoluminescence Spectroscopy for Nanomaterials Characterization, Springer Heidelberg New York Dordrecht London, p 179223, DOI 10.1007/978-3-642-27594-4_5 (2013).

\section{Comunicaciones a congresos}

1) I Reunión Conjunta AFA - SUF 2008, 93 ra Reunión NAcional DE Física Argentina AFA y XI Reunión de la Sociedad URuguaya DE FísICA - Departamento de Física de la Facultad de Ciencias Exactas y Naturales (FCEyN) de la Universidad de Buenos Aires (UBA), Ciudad Autónoma de Buenos Aires - Buenos Aires, 15 al 19 de Septiembre de 2008. 
"Función dieléctrica de nanopartículas de plata y aplicación a la determinación de resonancia plasmónica"

D. C. Schinca, J. M. J. Santillán y L. B. Scaffardi.

2) II Encuentro Nano Mercosur 2009: "Oportunidades de La Micro y NANOTECNOLOGÍA" - Fundación Argentina de Nanotecnología (FAN), Palacio San Miguel, Ciudad Autónoma de Buenos Aires - Buenos Aires, 4 al 6 de Agosto de 2009.

"Líneas de investigación en micro y nanotecnología"

Jorge O. Tocho, Lucía B. Scaffardi, Daniel C. Schinca, Gustavo A. Torchia, Fabián A. Videla y Jesica M. J. Santillán.

3) 94ta Reunión Nacional de Física AFA - Instituto de Física Rosario (IFIR) de la Universidad Nacional de Rosario (UNR), Rosario - Santa Fe, 14 al 18 de Septiembre de 2009.

"Resonancia plasmónica en nanopartículas de plata con recubrimiento de óxido. Análisis de tamaño por espectroscopía de extinción"

J. M. J. Santillán, L. B. Scaffardi, D. C. Schinca y G. A. Torchia.

4) 94 ta Reunión Nacional de Física AFA - Instituto de Física Rosario (IFIR) de la Universidad Nacional de Rosario (UNR), Rosario - Santa Fe, 14 al 18 de Septiembre de 2009.

"Sensor de gases contaminantes basado en Resonancia de Plasmón Superficial (SPR)”

J. M. J. Santillán, F. A. Videla, L. B. Scaffardi y D. C. Schinca. 
5) V TAller De Óptica y Fotofísica (Topfot): “Fotónica y Nuevos MAteriales" - Universidad CAECE, Mar del Plata - Buenos Aires, 19 y 20 de Noviembre de 2009.

“Resonancia plasmónica en nanopartículas de plata con recubrimiento de óxido. Análisis de tamaño por espectroscopía de extinción"

J. M. J. Santillán, L. B. Scaffardi, D. C. Schinca y G. A. Torchia.

6) SEMINARIOS DEL CIOP: “EsPeCtroscopía dE EXTINCIÓN PARA LA DETERMINACIÓN DEL TAMAÑO DE NANOPARTÍCULAS CORE-SHELL (Ag$\left.\boldsymbol{A g}_{2} \boldsymbol{O}\right)$ " - Centro de Investigaciones Ópticas (CIOp), Gonnet - Buenos Aires, 3 de Diciembre de 2009.

Jesica M. J. Santillán.

7) VI TAller de Óptica y Fotofísica (Topfot) - I Encuentro DE Estudiantes EN Óptica y Fotofísica (EEOF): “Óptica y Fotofísica EN DiAgNóstico MÉDiCo - CELEBRACión DE los 50 PRIMERos AÑos DEL LÁsER” - Campus de la Universidad Nacional del Centro de la Provincia de Buenos Aires (UNCPBA), Tandil - Buenos Aires, 26 al 28 de Mayo de 2010.

“Diseño de un sensor plasmónico tipo Kretschmann para la determinación de espesor de óxido de plata"

J. M. J. Santillán, F. A. Videla, D. C. Schinca y L. B. Scaffardi.

8) 10 th European Conference on "Atoms, Molecules And Photons", Palacio de Congresos, Salamanca - España, 4 al 9 de Julio de 2010.

“Optical spectroscopy extinction method for sizing noble metal nanoparticles generated by femtosecond laser ablation of solid in liquids" 
D. C. Schinca, L. B. Scaffardi, G. A. Torchia, F. A. Videla, J. M. J. Santillán, P. Moreno and L. Roso.

9) RIAO - OPTILAS 2010 "VII REunión IBEROAMERICANA DE ÓPTICA (RIAO) - X EnCUENTRO LATINOAMERICANO DE Óptica, LÁsERES Y APliCaciones (OPTILAS)", Departamento de Ciencias Sección Física de la Pontificia Universidad Católica del Perú (PUCP), San Miguel Lima, Perú, 20 al 24 de Septiembre de 2010.

"Surface Plasmon Resonance technique applied to measurement of $\mathrm{Ag}_{2} \mathrm{O}$ thin films"

Jesica M. J. Santillán, Lucía B. Scaffardi, Daniel C. Schinca and Fabián A. Videla.

10) $95^{\text {ta }}$ Reunión Nacional de Física AFA - Centro de Convenciones y Exposiciones "Thesaurus", Malargüe - Mendoza, 28 de Septiembre al 1 de Octubre de 2010.

"Método paramétrico basado en espectroscopía de extinción óptica para caracterización de nanopartícu-las aisladas coreshell de $\mathrm{Ag}-\mathrm{Ag}_{2} \mathrm{O}$ ”

J. M. J. Santillán, D. C. Schinca y L. B. Scaffardi.

11) $95^{\text {ta }}$ Reunión Nacional de Física AFA - Centro de Convenciones y Exposiciones "Thesaurus", Malargüe - Mendoza, 28 de Septiembre al 1 de Octubre de 2010.

"Diseño de un sensor óptico de mercurio basado en resonancia de plasmón superficial”

J. M. J. Santillán, L. B. Scaffardi y D. C. Schinca. 
12) VII TAller De Óptica y Fotofísica (Topfot) - II Encuentro de ESTUdiANTES EN ÓPTICA Y FOTOFÍSICA (EEOF): "METROLOGÍA ÓPTICA Y FotofísicA" - Dpto. de Luminotecnia, Luz y Visión de la Facultad de Ciencias Exactas y Tecnología (FACET) de la Universidad Nacional de Tucumán (UNT) e Instituto de Luz, Ambiente y Visión de la Universidad Nacional de Tucumán (UNT) - CONICET, San Miguel de Tucumán - Tucumán, 17 al 20 de Mayo de 2011.

“Caracterización óptica de nanoparticulas de Cu generadas por ablación láser de pulsos ultracortos en liquidos”

J. M. J. Santillán, L. B. Scaffardi, F. A. Videla y D. C. Schinca.

13) VII TAller de Óptica y Fotofísica (Topfot) - II EnCUentro DE

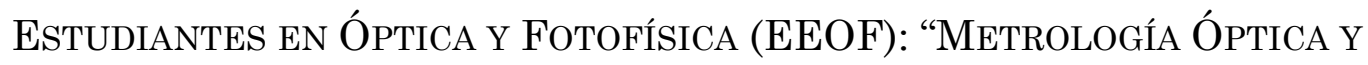
FotofísICA" - Dpto. de Luminotecnia, Luz y Visión de la Facultad de Ciencias Exactas y Tecnología (FACET) de la Universidad Nacional de Tucumán (UNT) e Instituto de Luz, Ambiente y Visión de la Universidad Nacional de Tucumán (UNT) - CONICET, San Miguel de Tucumán - Tucumán, 17 al 20 de Mayo de 2011.

"Análisis de la resonancia plasmónica de nanopartículas coreshell de Cu con distinta composición núcleo-cubierta"

F. A. Videla, D. C. Schinca, J. M. J. Santillán y L. B. Scaffardi.

14) XIII Giambiagi School: "Nanophotonics" - Departamento de Física de la Facultad de Ciencias Exactas y Naturales (FCEyN) de la Universidad Nacional de Buenos Aires (UBA), Ciudad Autónoma de Buenos Aires - Buenos Aires, 18 al 22 de Julio de 2011.

"A method for sizing core-shell $\mathrm{Ag}-\mathrm{Ag}_{2} \mathrm{O}$ single nanoparticle based on optical extinction spectroscopy"

J. M. J. Santillán, L. B. Scaffardi and D. C. Schinca. 
15) $2^{\text {nd }}$ EUlaSUR Summer School: "Simulation, Characterization AND Optical Methods FOR Materials AND NANOMATERIAls (Multifunctional Ceramics And Hybrids Materials)" - Centro de Investigaciones Ópticas (CIOp), Centro Científico Tecnológico La Plata (CONICET La Plata), La Plata - Buenos Aires, 4 al 9 de Septiembre de 2011.

“Optical extinction of core-shell metal nanoparticles generated by fs laser ablation. Analysis of free and bound electron contribution to plasmonic properties"

F. A. Videla, J. M. J. Santillán, D. C. Schinca and L. B. Scaffardi.

16) Encuentro NANo Mercosur 2011: "NANOteCnOlogía PARA LA INDUSTRIA Y LA SOCIEDAD” - Fundación Argentina de Nanotecnología (FAN), Palais Rouge, Ciudad Autónoma de Buenos Aires - Buenos Aires, 14 al 16 de Septiembre de 2011.

\section{"Líneas de investigación en micro y nanotecnología"}

Lucía B. Scaffardi, Daniel C. Schinca, Jorge O. Tocho, Gustavo A. Torchia, Álvaro D. Carrera, Juan A. Demarco, Jesica M. J. Santillán, Matías R. Tejerina y Fabián A. Videla.

17) II Reunión CONJUnta SUF - AFA 2011, XII REUnión DE LA SociedAD URuguaya DE FísicA y 96 ta Reunión NACIONAL DE FísicA AFA Instituto de Física de la Facultad de Ingeniería de la Universidad de la República, Edificio Polifuncional Ing. José Luis Massera (El Faro), Montevideo - Uruguay, 20 al 23 de Septiembre de 2011.

"Nanoparticulas core-shell de $\mathrm{Cu}-\mathrm{Cu}{ }_{2} \mathrm{O}$ : fabricación por ablación láser de pulsos ultracortos y caracterización óptica" J. M. J. Santillán, L. B. Scaffardi, F. A. Videla y D. C. Schinca. 
18) II REunión CONJUNTA SUF - AFA 2011, XII REUnión DE LA SoCIEDAD Uruguaya De Física y $96^{\text {ta }}$ Reunión Nacional de Física AFA Instituto de Física de la Facultad de Ingeniería de la Universidad de la República, Edificio Polifuncional Ing. José Luis Massera (El Faro), Montevideo - Uruguay, 20 al 23 de Septiembre de 2011.

"Simulación de espectros de extinción de nanopartículas coreshell de $\mathrm{Cu}-\mathrm{Cu}_{2} \mathrm{O}$ y $\mathrm{SiO}_{2}-\mathrm{Cu}$ ”

F. A. Videla, D. C. Schinca, J. M. J. Santillán y L. B. Scaffardi.

19) $2^{\text {nd }}$ EULASUR WORKSHOP - Copenhagen Business School, Copenhague, Dinamarca, 14 al 16 de Marzo de 2012.

“Participation of CIOp (CONICET La Plata-CIC) at EULASUR Project"

Federico E. Sanjuan, Jesica M. J. Santillán, Lucía B. Scaffardi, Daniel C. Schinca, Jorge O. Tocho, Gustavo A. Torchia, Marcelo R. Trivi and Fabián A. Videla.

20) VIII TALler DE ÓpticA y Fotofísica (Topfot) - III ENCUENTRO DE ESTUdIANTES EN ÓPTICA Y FotofísICA (EEOF): “ÓPTICA Y FoTÓNICA EN SISTEMAS AEROESPACIALES" - Centro de Investigaciones Ópticas (CIOp), Centro Científico Tecnológico La Plata (CONICET La Plata), La Plata - Buenos Aires, 21 al 24 de Mayo de 2012.

"Influencia de los electrones ligados en la función dieléctrica de Cu y en los espectros plasmónicos subnanométricos”

J. M. J. Santillán, L. B. Scaffardi, F. A. Videla y D. C. Schinca.

21) SPIE Optics + Photonics: "NAnoScience + Enginneering" - San Diego Convention Center, San Diego - California, USA, 11 al 16 de Agosto de 2012. 
"Plasmonic properties and sizing of core-shell $\mathrm{Cu}-\mathrm{Cu}{ }_{2} \mathrm{O}$ nanoparticles fabricated by femtosecond laser ablation in liquids"

J. M. J. Santillán, F. A. Videla, D. C. Schinca and L. B. Scaffardi.

22) 97a Reunión Nacional de la Asociación Física Argentina AFA Facultad de Matemática, Astronomía y Física (FaMAF) de la Universidad Nacional de Córdoba (UNC), Hotel Portal del Lago, Villa Carlos Paz - Córdoba, 25 al 28 de Septiembre de 2012.

"Función dieléctrica de plata dependiente del tamaño. Determinación de parámetros de banda y radio de nanopartículas generadas por ablación láser"

Jesica M. J. Santillán, Daniel C. Schinca, Fabián A. Videla y Lucía B. Scaffardi.

23) $2^{\text {da }}$ Reunión NanoCóRdoba 2012 - Universidad de Córdoba (UNC), Hotel Portal del Lago, Villa Carlos Paz - Córdoba, 1 al 3 de Octubre de 2012 .

"Nanopartículas de Cu, Ag y Au producidas por ablación en líquidos con láser de pulsos ultracortos. Estructura y mecanismos de fragmentación"

D. C. Schinca, F. A. Videla, J. M. J. Santillán y L. B. Scaffardi.

24) Latin America Optics \& Photonics Conference (LAOP) - The Optical Society (OSA), Maresias Beach Hotel, San Sebastián, Brasil, 10 al 13 de Noviembre de 2012. 
"Spectroscopic approach to structure, configuration and size determination of $\mathrm{Cu}$ nanoparticles generated by fs laser ablation in liquids"

J. M. J. Santillán, F. A. Videla, D. C. Schinca and L. B. Scaffardi. 


\section{Bibliografía}

[Abraham Ekeroth et al. 2011] Abraham Ekeroth R. M., Lester M., Scaffardi L. B. and Schinca D. C., Metallic Nanotubes Characterization via Surface Plasmon Excitation, Plasmonics 6 (3), 435-444 (2011).

[Alivisatos et al. 1998] Alivisatos P. A., Barbara P. F., Castleman A. W., Chang J., Dixon D. A., Klein M. L., McLendon G. L., Miller J. S., Ratner M. A., Rossky J. P., Stupp S. I., and Thompson M. E., From Molecules to Materials: Current Trends and Future Directions, Adv. Mater. 10 (16), 1297-1336 (1998).

[Amikura et al. 2008] Amikura K., Kimura T., Hamada M., Yokoyama N., Miyazaki J. and Yamada Y., Copper oxide particles produced by laser ablation in water, Appl. Surface Science 254 (21), 6976-6982 (2008).

[Amoruso et al. 2005] Amoruso S., Ausanio G., Bruzzese R., Vitiello M. and Wang X., Femtosecond laser pulse irradiation of solid targets as a general route to nanoparticle formation in a vacuum, Phys. Rev. B $\mathbf{7 1}$ (3), 033406 (2005).

[Anker et al. 2008] Anker J. N., Paige Hall W., Lyandres O., Shah N. C., Zhao J. and Van Duyne R. P., Biosensing with plasmonic nanosensors, Nature Mater. 7 (6), 442-453 (2008). 
[Apell and Penn 1983] Apell P. and Penn D. R., Optical Properties of Small Metal Spheres: Surface Effects, Phys. Rev. Lett. 50 (17), 1316-1319 (1983).

[Ashcroft and Mermin 1976] Ashcroft N. W. and Mermin N. D., Solid State Physics, Saunders College Publishing, Orlando (1976).

[Baida et al. 2009] Baida H., Billaud P., Marhaba S., Christofilos D., Cottancin E., Crut A., Lermé J., Maioli P., Pellarin M., Broyer M., Del Fatti N. and Vallée F., Quantitative Determination of the Size Dependence of Surface Plasmon Resonance Damping in Single Ag@ $@ \mathrm{SiO}_{2}$ Nanoparticles, Nano Lett. 9 (10), 3463-3469 (2009).

[Barcikowski et al. 2007a] Barcikowski S., Hahn A., Kabashin A. V. and Chichkov B. N., Properties of nanoparticles generated during femtosecond laser machining in air and water, Appl. Phys. A 87 (1), 47 55 (2007).

[Barcikowski et al. 2007b] Barcikowski S., Menéndez-Manjón A., Chichkov B. N., Brikas M. and Račiukaitis G., Generation of nanoparticle colloids by picosecond and femtosecond laser ablation in liquid flow, Appl. Phys. Lett. 91 (8), 083113 (2007).

[Ben Moshe and Markovich 2011] Ben Moshe A. and Markovich G., Synthesis of Single Crystal Hollow Silver Nanoparticles in a Fast Reaction-Diffusion Process, Chem. Mater. 23 (5), 1239-1245 (2011).

[Besner et al. 2007] Besner S., Kabashin A. V. and Meunir M., Two-step femtosecond laser ablation-based method for the synthesis of stable and ultra-pure gold nanoparticles in water, Appl. Phys. A 88 (2), 269272 (2007). 
[Billaud et al. 2007] Billaud P., Huntzinger J. R., Cottancin E., Lermé J., Pellarin M., Arnaud L., Broyer M., Del Fatti N. and Vallée F., Optical extinction spectroscopy of single silver nanoparticles, Eur. Phys. J. D 43 (1), 271-274 (2007).

[Bogatyrev et al. 2004] Bogatyrev V. A., Dykman L. A., Khlebtsov B. N. and Khlebtsov N. G., Measurement of Mean Size and Evaluation of Polydispersity of Gold Nanoparticles from Spectra of Optical Absorption and Acattering, Opt. Spectrosc. 96 (1), 128-135 (2004).

[Bohren and Huffman 1998] Bohren C. F. and Huffman D. R., Absorption and Scattering of Light by Small Particles, John Wiley \& Sons, New York (1998).

[Bonacic-Koutecky et al. 1991] Bonacic-Koutecky V., Fantucci P. and Koutecky J., Quantum chemistry of small clusters of elements of groups Ia, Ib, and IIa: fundamental concepts, predictions, and interpretation of experiments, Chem. Rev. 91 (5), 1035-1108 (1991).

[Boyen et al. 2002] Boyen H. G., Kästle G., Weigl F., Koslowski B., Dietrich C., Ziemann P., Spatz J. P., Riethmüller S., Hartmann C., Möller M., Schmid G., Garnier M. G. and Oelhafen P., Oxidation-Resistant Gold55 Clusters, Science 297 (5586), 1533-1536 (2002).

[Brack 1993] Brack M., The physics of simple metal clusters: self-consistent jellium model and semiclassical approaches, Rev. Mod. Phys. 65 (3), 677-732 (1993).

[Cai and Shalaev 2010] Cai W. and Shalaev V., Optical metamaterials. Fundamentals and applications, Springer, New York (2010). 
[Cao et al. 2002] Cao Y. W. C., Jin R. and Mirkin C. A., Nanoparticles with Raman Spectroscopic Fingerprints for DNA and RNA Detection, Science 297 (5586), 1536-1540 (2002).

[Chatterjee et al. 2002] Chatterjee K, Banerjee S. and Chakravorty D., Plasmon resonance shifts in oxide-coated silver nanoparticles, Phys. Rev. B 66 (8), 085421-7 (2002).

[Chau et al. 2006] Chau L.-K., Lin Y.-F., Cheng S.-F. and Lin T-J, Fiberoptic chemical and biochemical probes based on localized surface plasmon resonance, Sensors and Actuators B 113 (1), 100-105 (2006).

[Coronado et al. 2003] Coronado E. A. and Schatz G. C., Surface plasmon broadening for arbitrary shape nanoparticles: A geometrical probability approach, J. Chem. Phys. 119 (7), 3926-3934 (2003).

[de Bruijn et al. 1991] de Bruijn H. E., Altenburg B. S. F., Kooyman R. P. H. and Greve J., Determination of thickness and dielectric constant of thin transparent dielectric layers using Surface Plasmon Resonance, Opt. Commun. 82 (5-6), 425-432 (1991).

[Desarkar et al. 2013] Desarkar H. S., Kumbhakar P. and Mitra A K, Onestep synthesis of $\mathrm{Zn} / \mathrm{ZnO}$ hollow nanoparticles by the laser ablation in liquid technique, Laser Phys. Lett. 10 (5), 055903-055911 (2013).

[Dieringer et al. 2006] Dieringer J. A., McFarland A. D., Shah N. C., Stuart D. A., Whitney A. V., Yonzon C. R., Young M. A., Zhang X. and Van Duyne R. P., Introductory Lecture Surface enhanced Raman spectroscopy: new materials, concepts, characterization tools, and applications, Faraday Discuss 132, 9-26 (2006). 
[Dolgaev et al. 2002] Dolgaev S. I., Simakin A. V., Voronov V. V., Shafeev G. A. and Bozon-Verduraz F., Nanoparticles produced by laser ablation of solids in liquid environment, Appl. Surf. Science 186 (1-4), 546-551 (2002).

[Doyle 1958] Doyle W. T., Absorption of Light by Colloids in Alkali Halide Crystals, Phys. Rev. 111 (4), 1067-1072 (1958).

[Drude 1900a] Drude P., Zur Ionentheorie der Metalle, Physikalische Zeitschrift 1, 161-165 (1900).

[Drude 1900b] Drude P., Zur Elektronentheorie der Metalle, Ann. Phys. 306 (3), 566-613 (1900).

[Ebbesen et al. 1998] Ebbesen T. W., Lezec H. J., Ghaemi H. F., Thio T. and Wolff P. A., Extraordinary optical transmission through subwavelength hole arrays, Nature 391, 667-669 (1998).

[Elechiguerra et al. 2005] Elechiguerra J. L., Burt J. L., Morones J. R., Camacho-Bragado A., Gao X., Lara H. H. and Yacaman M. J., Interaction of silver nanoparticles with HIV-1, J. Nanobiotechnol., doi:10.1186/1477-155-3-6 (2005).

[Evanoff et al. 2004] Evanoff D. D., White R. L. and Chumanov G., Measuring the Distance Dependence of the Local Electromagnetic Field from Silver Nanoparticles, J. Phys. Chem. B 108 (5), 1522-1524 (2004).

[Fano 1941] Fano U., The Theory of Anomalous Diffraction Gratings and of Quasi-Stationary Waves on Metallic Surfaces (Sommerfeld's Waves), J. Opt. Soc. Am. 31 (3), 213-222 (1941). 
[Fox 2001] Fox M., Optical Properties of Solids, Oxford University Press, New York (2001).

[Fuji et al. 2000] Fuji H., Tominaga J., Men L., Nakano T., Katayama H. and Atoda N., A Near-Field Recording and Readout Technology Using a Metallic Probe in an Optical Disk, Jpn. J. Appl. Phys. 39 (2B), 980981 (2000).

[Fujimaki et al. 2006] Fujimaki M., Awazu K., Tominaga J. and Iwanabe Y., Surface-enhanced Raman scattering from Ag nanoparticles formed by visible laser irradiation of thermally annealed $\mathrm{AgO}_{x}$ thin films, J. Appl. Phys. 100 (7), 074303-074308 (2006).

[Garcés Vernier et al. 2000] Garcés Vernier I., Sotolongo O., Hernández M. P., Scaffardi L., García-Ramos J. V. and Rivas L., Determination of particle size distribution of particles on aerosols and suspensions by ultraviolet-visible-near infrared absorbance measurements. A new procedure for absorbing particles, Physica Status Solidi B 220 (1), 583586 (2000).

[Gao et al. 2004] Gao X., Wang S., Li J., Zheng Y., Zhang R., Zhou P., Yang Y. and Chen L., Study of structure and optical properties of silver oxide films by ellipsometry, XRD and XPS methods, Thin Solid Films 455456, 438-442 (2004).

[Genzel et al. 1975] Genzel L., Martin T. P. and Kreibig U., Dielectric function and plasma resonances of small metal particles, Z. Physik B 21 (4), 339-346 (1975). 
[Ghilane et al. 2007] Ghilane J., Fan F.-R. F., Bard A. J. and Dunwoody N., Facile Electrochemical Characterization of Core/Shell Nanoparticles. $\mathrm{Ag}$ Core $/ \mathrm{Ag}_{2} \mathrm{O}$ Shell Structures, Nano Lett. 7 (5), 1406-1412 (2007).

[Ghodselahi and Vesaghi 2011] Ghodselahi T. and Vesaghi M. A., Localized Surface Plasmon Resonance of $\mathrm{Cu} @ \mathrm{Cu}_{2} \mathrm{O}$ core-shell nanoparticles: Absorption, Scattering and Luminiscence, Physica B: Condensed Matter 406 (13), 2678-2683 (2011).

[Giannini et al. 2011] Giannini V., Fernández-Domínguez A. I., Heck S. C. and Maier S. A., Plasmonic nanoantennas: fundamentals and their use in controlling the radiative properties of nanoemitters, Chem. Rev. 111 (6), 3888-3912 (2011).

[Gotoh et al. 2000] Gotoh Y., Igarashi R., Ohkoshi Y., Nagura M., Akamatsu K., and Deki S., Preparation and structure of copper nanoparticle/poly(acrylicacid) composite films, J. Mater. Chem. 10 (11), 2548-2552 (2000).

[Grady et al. 2004] Grady N. K., Halas N. J. and Nordlander P., Influence of dielectric function properties on the optical response of plasmon resonant metallic nanoparticles, Chem. Phys. Lett. 399 (1-3), 167-171 (2004).

[Granqvist and Hunderi 1977] Granqvist C. G. and Hunderi O., Optical properties of ultrafine gold particles, Phys. Rev. B 16 (8), 3513-3534 (1977).

[Grojo et al. 2005] Grojo D., Hermann J. and Perrone A., Plasma analyses during femtosecond laser ablation of Ti, Zr and Hf, J. Appl. Phys. 97 (6), 063306 (2005). 
[Haes et al. 2004] Haes A. J., Hall W. P., Chang L., Klein W. L. and Van Duyne R. P., A Localized Surface Plasmon Resonance Biosensor: First Steps toward an Assay for Alzheimer's Disease, Nano Lett. 4 (6), 10291034 (2004).

[Hirsch et al. 2003a] Hirsch L. R., Jackson J. B., Lee A., Halas N. J. and West J. L., A Whole Blood Immunoassay Using Gold Nanoshells, Anal. Chem. 75 (10), 2377-2381 (2003).

[Hirsch et al. 2003b] Hirsch L. R., Stafford R. J., Bankson J. A., Sershen S. R., Rivera B., Price R. E., Hazle J. D., Halas N. J. and West J. L., Nanoshell-mediated near-infrared thermal therapy of tumors under magnetic resonance guidance, Proc. Nat. Acad. Sci. 100 (23), 1354913554 (2003).

[Homola 2008] Homola J., Surface Plasmon Resonance Sensors for Detection of Chemical and Biological Species, Chem. Rev. 108 (2), 462-493 (2008).

[Homola et al. 1999] Homola J., Yee S. S. and Gauglitz G., Surface plasmon resonance sensors: review, Sensors and Actuators B 54 (1-2), 3-15 (1999).

[Hou et al. 1998] Hou S. M., Ouyang M., Chen H. F., Liu W. M., Xue Z. Q., Wu Q. D., Zhang H. X., Gao H. J. and Pang S. J., Fractal structure in the silver oxide thin film, Thin Solid Films 315 (1-2), 322-326 (1998).

[Inouye et al. 1998] Inouye H., Tanaka K., Tanahashi I. and Hirao K., Ultrafast dynamics of nonequilibrium electrons in a gold nanoparticles system, Phys. Rev. B 57 (18), 11334-11340 (1998). 
[Jiang et al. 2003] Jiang J., Bosnick K., Maillard M. and Brus L., Single Molecule Raman Spectroscopy at the Junctions of Large Ag Nanocrystals, J. Phys. Chem. B 107 (37), 9964-9972 (2003).

[Johnson and Christy 1972] Johnson P. B. and Christy R. W., Optical Constants of Noble Metals, Phys. Rev. B 6 (12), 4370-4379 (1972).

[Kabashin and Meunier 2006] Kabashin A. V. and Meunier M., Laser ablation-based synthesis of nanomaterials, in Recent Advances in laser processing of materials Ed. J. Perriere, E. Million and E. Fogarassy, Elsevier (2006).

[Kabashin and Meunier 2007] Kabashin A. V. and Meunier M., Femtosecond laser ablation in aqueous solutions: a novel method to synthesize nontoxic metal colloids with controllable size, J. Phys.: Conf. Ser. 59 (1), 354-359 (2007).

[Karavanskii et al. 2004] Karavanskii V. A., Simakin A. V., Krasovskii V. I. and Ivanchenko P. V., Nonlinear optical properties of colloidal silver nanoparticles produced by laser ablation in liquids, Quantum Electron. 34 (7), 644-648 (2004).

[Kawasaki 2011] Kawasaki M., Laser-Induced Fragmentative Decomposition of Fine CuO Powder in Acetone as Highly Productive Pathway to $\mathrm{Cu}$ and $\mathrm{Cu}_{2} \mathrm{O}$ Nanoparticles, J. Phys. Chem. C 115 (12), 5165-5173 (2011).

[Kaye and Laby 1995] Kaye G. W. C. and Laby T. H., Tables of physical and chemical constants and some mathematical functions, Longman Scientific and Technical, London (1995). 
[Kazakevich et al. 2006] Kazakevich P. V., Simakin A. V., Voronov V. V. and Shafeev G. A., Laser induced synthesis of nanoparticles in liquids, Appl. Surf. Science 252 (13), 4373-4380 (2006).

[Kelly et al. 2003] Kelly K. L., Coronado E., Zhao L. L. and Schatz G. C., The Optical Properties of Metal Nanoparticles: The Influence of Size, Shape, and Dielectric Environment, J. Phys. Chem. B 107 (3), 668-677 (2003).

[Kim et al. 2007] Kim K. T., Song H. S., Mah J. P., Hong K. B., Im K., Baik S.-J. and Yoon Y.-I., Hydrogen Sensor Based on Palladium Coated Side-Polished Single-Mode Fiber, Sensors Journal IEEE 7 (12), 17671771 (2007).

[Kittel 2007] Kittel C., Introduction to solid state physics, John Wiley \& Sons, New York (2007).

[Klar 2007] Klar T. A., Chapter 8: Biosensing with plasmonic nanoparticles en el libro "Nanophotonics with surface plasmons, editado por Shalaev V. M. y Kawata S., Elveiser, Oxford (2007).

[Kneipp et al. 2010] Kneipp J., Kneipp H., Wittig B. and Kneipp K., Novel optical nanosensors for probing and imaging live cells, Nanomedicine 6 (2), 214-226 (2010).

[Knoll 1998] Knoll W., Interfaces and thin films as seen by bound electromagnetic waves, Annual Review of Physical Chemistry 49, 569638 (1998).

[König et al. 2005] König J., Nolte S. and Tünnermann A., Plasma evolution during metal ablation with ultrashort laser pulses, Optics Express 13 (26), 10597-10607 (2005). 
[Kottmann and Martin 2001] Kottmann J. P. and Martin O. J. F., Influence of the cross section and the permittivity on the plasmon-resonance spectrum of silver nanowires, Appl. Phys. B 73 (4), 299-304 (2001).

[Kraus and Schatz 1983a] Kraus W. A. and Schatz G. C., Plasmon resonance broadening in small metal particles, J. Chem. Phys. 79 (12), 6130-6139 (1983).

[Kraus and Schatz 1983b] Kraus W. A. and Schatz G. C., Plasmon resonance broadening in spheroidal metal particles, Chem. Phys. Lett. 99 (4), 353357 (1983).

[Kreibig 1970] Kreibig U., Kramers Kronig analysis of the optical properties of small silver particles, Zeitschrift für Physik 234 (4), 307-318 (1970).

[Kreibig and Vollmer 1995] Kreibig U. and Vollmer M., Optical Properties of Metal Clusters, Springer, Berlin (1995).

[Kreibig and von Fragstein 1969] Kreibig U. and von Fragstein C., The limitation of electron mean free path in small silver particles, Zeitschrift für Physik 224 (4), 307-323 (1969).

[Kretschmann and Raether 1968] Kretschmann E. and Raether H., Radiative decay of non-radiative surface plasmons excited by light, $Z$. Naturforschung A 23 (12), 2135-2136 (1968).

[Larsen and Noriega 2004] Larsen G. and Noriega S., Dendrimer-mediated formation of $\mathrm{Cu}-\mathrm{CuO}_{x}$ nanoparticles on silica and their physical and catalytic characterization, Appl. Catal. A: Gen. 278 (1), 73-81 (2004). 
[Li et al. 2003] Li K., Stockman M. I. and Bergman D. J., Self-Similar Chain of Metal Nanospheres as an Efficient Nanolens, Phys. Rev. Lett. 91 (22), 227402 (2003).

[Li et al. 2005] Li Y., Moon K.-S. and Wong C. P., Monolayer protected silver nanoparticle-based anisotropic conductive adhesives: Enhancement of electrical and thermal properties, J. Electron. Mater. 34 (12), 15731578 (2005).

[Libardi and Grieneisen 1998] Libardi H. and Grieneisen H. P., Guidedmode resonance absorption in partly oxidized thin silver films, Thin Solid Films 333 (1-2), $82-87$ (1998).

[Liedberg et al. 1983] Liedberg B., Nylander C. and Lundström I., Surfaceplasmon resonance for gas-detection and biosensing, Sensors and Actuators 4, 299-304 (1983).

[Link and El-Sayed 2000] Link S. and El-Sayed M. A., Shape and size dependence of radiative, non-radiative and photothermal properties of gold nanocrystals, Int. Rev. Phys. Chem. 19 (3), 409-453 (2000).

[Liu et al. 2011] Liu P., Li Z., Cai W., Fang M. and Luo X., Fabrication of cuprous oxide nanoparticles by laser ablation in PVP aqueous solution, RSC Advances 1 (5), 847-851 (2011).

[Logunov et al. 1997] Logunov S. L., Ahmadi T. S., El-Sayed M. A., Khoury J. T. and Whetten R. L., Electron Dynamics of Passivated Gold Nanocrystals Probed by Subpicosecond Transient Absorption Spectroscopy, J. Phys. Chem. B 101, 3713-3719 (1997). 
[Lorentz 1905] Lorentz H. A., The motion of electrons in metallic bodies I, II and III, Proc. K. Akad. Amst. 7, 438-453, 585-593, 684-691 (1905).

[McFarland and Van Duyne 2003] McFarland A. D. and Van Duyne R. P., Single Silver Nanoparticles as Real-Time Optical Sensors with Zeptomole Sensitivity, Nano Lett. 3 (8), 1057-1062 (2003).

[Mafuné et al. 2000a] Mafuné F., Kohno J., Takeda Y. and Kondow T., Structure and Stability of Silver Nanoparticles in Aqueous Solution Produced by Laser Ablation, J. Phys. Chem. B 104 (35), 8333-8337 (2000).

[Mafuné et al. 2000b] Mafuné F., Kohno J., Takeda Y. and Kondow T., Formation and Size Control of Silver Nanoparticles by Laser Ablation in Aqueous Solution, J. Phys. Chem. B 104 (39), 9111-9117 (2000).

[Mafuné et al. 2002a] Mafuné F., Kohno J., Takeda Y. and Kondow T., Full Physical Preparation of Size-Selected Gold Nanoparticles in Solution: Laser Ablation and Laser-Induced Size Control, J. Phys. Chem. B 106 (31), 7575-7577 (2002).

[Mafuné et al. 2002b] Mafuné F., Kohno J., Takeda Y. and Kondow T., Growth of Gold Clusters into Nanoparticles in a Solution Following Laser-Induced Fragmentation, J. Phys. Chem. B 106 (34), 8555-8561 (2002).

[Mafuné et al. 2003] Mafuné F., Kohno J., Takeda Y. and Kondow T., Formation of Stable Platinum Nanoparticles by Laser Ablation in Water, J. Phys. Chem. B 107 (18), 4218-4223 (2003). 
[Maier 2007] Maier S. A., Plasmonics: Fundamentals and Applications, Springer, Berlin (2007).

[Maier et al. 2003] Maier S. A., Kik P. G., Atwater H. A., Meltzer S., Harel E., Koel B. E., Requicha A. A. G., Local detection of electromagnetic energy transport below the diffraction limit in metal nanoparticle plasmon waveguides, Nat. Mater. 2 (4), 229-232 (2003).

[Mie 1908] Mie G., Beiträge zur Optik Trüber Medien, Speziell Kolloidaler Metall-Lösungen, Ann. Phys. 25, 377-445 (1908).

[Moran et al. 2005] Moran A. M., Sung J., Hicks E. M., Van Duyne R. P. and Spears K. G., Second Harmonic Excitation Spectroscopy of Silver Nanoparticle Arrays, J. Phys. Chem. B 109 (10), 4501-4506 (2005).

[Murphy 2009] Murphy M. P., How mitochondria produce reactive oxygen species, Biochem. J. 417, 1-13 (2009).

[Muskens et al. 2008] Muskens O. L., Billaud P., Broyer M., Del Fatti N. and Vallée F., Optical extinction spectrum of a single metal nanoparticle: Quantitative characterization of a particle and of its local environment, Phys. Rev. B 78 (20), 205410 (2008).

[Nath and Chilkoti 2002] Nath N. and Chilkoti A., A Colorimetric Gold Nanoparticle Sensor To Interrogate Biomolecular Interactions In Real Time On A Aurface, Anal. Chem. 74 (3), 504-509 (2002).

[Nath and Khare 2011] Nath A. and Khare A., Size induced structural modifications in copper oxide nanoparticles synthesized via laser ablation in liquids, J. Appl. Phys. 110 (4), 043111 (2011). 
[Novo et al. 2008] Novo C., Funston A. M. and Mulvaney P., Direct observation of chemical reactions on single gold nanocrystals using surface plasmon spectroscopy, Nat. Nanotechnol. 3 (10), 598-602 (2008).

[Novo et al. 2009] Novo C., Funston A. M., Gooding A. K. and Mulvaney P., Electrochemical Charging of Single Gold Nanorods, J. Am. Chem. Soc. 131 (41), 14664-14666 (2009).

[Novotny and Hecht 2007] Novotny L. and Hecht B., Principles of NanoOptics, Cambridge University Press, New York (2007).

[Palik 1985] Palik E. D., Handbook of Optical Constants of Solids, Academic Press, New York (1985).

[Patel et al. 2005] Patel M. K., Nagare B. J., Bagul D. M., Haram S. K. and Kothari D. C., Controlled synthesis of $\mathrm{Cu}$ nanoparticles in fused silica and BK7 glasses using ion beam induced defects, Surf. Coat. Technol. 196 (1-3), 96-99 (2005).

[Pedersen et al. 2008] Pedersen D. B., Wang S. and Liang S. H., ChargeTransfer-Driven Diffusion Processes in $\mathrm{Cu} @ \mathrm{Cu}$-Oxide Core-Shell Nanoparticles: Oxidation of $3.0 \pm 0.3 \mathrm{~nm}$ Diameter Copper Nanoparticles, J. Phys. Chem. C 112, 8819-8826 (2008).

[Pettersson and Snyder 1995] Pettersson L. A. A. and Snyder P. G., Preparation and characterization of oxidized silver thin films, Thin Solid Films 270 (1-2), 69-72 (1995).

[Peyser et al. 2001] Peyser L. A., Vinson A. E., Bartko A. P. and Dickson R. M., Photoactivated Fluorescence from Individual Silver Nanoclusters, Science 291 (5501), 103-106 (2001). 
[Pike et al. 2006] Pike J., Chan S. W., Zhang F., Wang X. and Hanson J., Formation of stable $\mathrm{Cu}_{2} \mathrm{O}$ from reduction of $\mathrm{CuO}$ nanoparticles, Appl. Catal. A: Gen. 303 (2), 273-277 (2006).

[Pinchuk et al. 2004] Pinchuk A., von Plessen G. and Kreibig U., Influence of interband electronic transitions on the optical absorption in metallic nanoparticles, J. Phys. D: Appl. Phys. 37 (22), 3133-3139 (2004).

[Poderoso et al. 1999] Poderoso J. J., Lisdero C., Schöpfer F., Riobó N., Carreras M. C., Cadenas E. and Boverisi A., The Regulation of Mitochondrial Oxygen Uptake by Redox Reactions Involving Nitric Oxide and Ubiquinol, J. Biol. Chem. 274 (53), 37709-37716 (1999).

[Prodan et al. 2003] Prodan E., Nordlander P. and Halas N. J., Electronic Structure and Optical Properties of Gold Nanoshells, Nano Lett. 3 (10), 1411-1415 (2003).

[Qiu et al. 2005] Qiu J. H., Zhou P., Gao X. Y., Yu J. N., Wang S. Y., Li J., Zheng Y. X., Yang Y. M., Song Q. H. and Chen L. Y., Ellispsometric Study of the Optical Properties of Silver Oxide Prepared by Reactive Magnetron Sputtering, J. Korean Phys. Soc. 46, S269-S275 (2005).

[Quaranta et al. 2004] Quaranta A., Ceccato R., Menato C., Pederiva L., Capra N., and Dal Maschio R., Formation of copper nanocrystals in alkali-lime silica glass by means of different reducing agents, J. NonCryst. Solids 345-346 (10), 671-675 (2004).

[Rahn and Hallock 1995] Rahn J. R. and Hallock R. B., Antibody Binding to Antigen-Coated Substrates Studied with Surface Plasmon Oscillations, Langmuir 11 (2), 650-654 (1995). 
[Raju et al. 2009] Raju N. R. C., Kumar K. J. and Subrahmanyam A., Physical properties of silver oxide thin films by pulsed laser deposition: effect of oxygen pressure during growth, J. Phys. D: Appl. Phys. 42 (13), 135411-135417 (2009).

[Ramakrishnan et al. 2005] Ramakrishnan A., Yongqiang T. and Sadana A., A kinetic study of analyte-receptor binding and dissociation for surface plasmon resonance biosensors applications, Sensors Journal IEEE 5 (3), 356-364 (2005).

[Ranjan et al. 2010] Ranjan M., Oates T. W., Facsko S. and Möller W., Optical properties of silver nanowire arrays with $35 \mathrm{~nm}$ periodicity, Opt. Lett. 35 (15), 2576-2578 (2010).

[Riboh et al. 2003] Riboh J. C., Haes A. J., McFarland A. D., Yonzon C. R. and Van Duyne R. P., A Nanoscale Optical Biosensor: Real-Time Immunoassay in Physiological Buffer Enabled by Improved Nanoparticle Adhesion, J. Phys. Chem. B 107 (8), 1772-1780 (2003).

[Ritchie 1957] Ritchie R. H., Plasma Losses by Fast Electrons in Thin Films, Phys. Rev. 106 (5), 874-881 (1957).

[Roldán et al. 2008] Roldán M. V., Scaffardi L. B., de Sanctis O. and Pellegri N., Optical properties and extinction spectroscopy to characterize the synthesis of amine capped silver nanoparticles, Mater. Chem. Phys. 112 (3), 984-990 (2008).

[Rosei 1974] Rosei R., Temperature modulation of the optical transitions involving the Fermi surface in Ag: Theory, Phys. Rev. B 10 (2), 474-483 (1974). 
[Rosei et al. 1973] Rosei R., Antonangeli F. and Grassano U. M., d bands position and width in gold from very low temperature thermomodulation measurements, Surf. Sci. 37, 689-699 (1973).

[Rostovshchikova et al. 2005] Rostovshchikova T. N., Smirnov V. V., Kozhevin V. M., Yavsin D. A., Zabelin M. A., Yassievich I. N. and Gurevich S. A., New size effect in the catalysis by interacting copper nanoparticles, Appl. Catal. A: Gen. 296 (1), 70-79 (2005).

[Ruppin and Yatom 1976] Ruppin R. and Yatom H., Size and Shape Effects on the Broadening of the Plasma Resonance Absorption in Metals, Phys. Status Solidi B 74 (2), 647-654 (1976).

[Sahm et al. 2004] Sahm H., Charton C. and Thielsch R., Oxidation behaviour of thin silver films deposited on plastic web characterized by spectroscopic ellipsometry (SE), Thin Solid Films 455-456, 819-823 (2004).

[Santillán et al. 2010] Santillán J. M. J., Scaffardi L. B., Schinca D. C. and Videla F. A., Determination of nanometric $\mathrm{Ag}_{2} \mathrm{O}$ film thickness by surface plasmon resonance and optical waveguide mode coupling techniques, J. Opt. 12 (4), 045002-045009 (2010).

[Santillán et al. 2011] Santillán J. M. J., Scaffardi L. B. and Schinca D. C., Quantitative optical extinction-based parametric method for sizing a single core-shell $\mathrm{Ag}-\mathrm{Ag}_{2} \mathrm{O}$ nanoparticle, J. Phys. D: Appl. Phys. 44 (10), 105104-105111 (2011).

[Santillán et al. 2012a] Santillán J. M. J., Videla F. A., Scaffardi L. B. and Schinca D. C., Plasmon Spectroscopy for Subnanometric Copper Particles: Dielectric Function and Core-Shell Sizing, Plasmonics, doi: 10.1007/s11468-012-9395-8 (2012). 
[Santillán et al. 2012b] Santillán J. M. J., Videla F. A., Fernández van Raap M. B., Schinca D. C. and Scaffardi L. B., Size dependent $\mathrm{Cu}$ dielectric function for plasmon spectroscopy: Characterization of colloidal suspension generated by fs laser ablation, J. Appl. Phys. 112 (5), 054319-054326 (2012).

[Santillán et al. 2012c] Santillán J. M. J., Videla F. A., Schinca D. C. and Scaffardi L. B., Plasmonic properties and sizing of core-shell $\mathrm{Cu}-\mathrm{Cu}_{2} \mathrm{O}$ nanoparticles fabricated by femtosecond laser ablation in liquids, Proc. SPIE 8457, Plasmonics: Metallic Nanostructures and Their Optical Properties X, 84572U, doi: 10.1117/12.928670 (2012).

[Santillán et al. 2013a] Santillán J. M. J., Videla F. A., Fernández van Raap M. B., Schinca D. C. and Scaffardi L. B., Analysis of the structure, configuration, and sizing of $\mathrm{Cu}$ and $\mathrm{Cu}$ oxide nanoparticles generated by fs laser ablation of solid target in liquids, J. Appl. Phys. 113 (13), 134305-134313 (2013).

[Santillán et al. 2013b] Santillán J. M. J., Videla F. A., Fernández van Raap M. B., Muraca D., Scaffardi L. B. and Schinca D. C., Influence of sizecorrected bound-electron contribution on nanometric silver dielectric function. Sizing through optical extinction spectroscopy, en redacción (2013).

[Santillán et al. 2013c] Santillán J. M. J., Fernández van Raap M. B., Muraca D., Mendoza Zelis P., Videla F. A., Schinca D. C. and Scaffardi L. B., Self assembled fractal-like nanostructures formed by silver nanoparticles fabricated using ultrashort pulse laser ablation, en redacción (2013). 
[Scaffardi and Tocho 2006] Scaffardi L. B. and Tocho J. O., Size dependence of refractive index of gold nanoparticles, Nanotechnology 17 (5), 13091315 (2006).

[Scaffardi and Tocho 2008] Scaffardi L. B. and Tocho J. O., Absorption spectra of tiny gold and silver objects, Journal of Luminiscence $\mathbf{1 2 8}$ (5-6), 828-830 (2008).

[Scaffardi et al. 1996] Scaffardi L., Tocho J. O., Yebrin L. and Cantera C., Sizing particles used in the leather industry by light scattering, Optical Engineering 35 (1), 52-56 (1996).

[Scaffardi et al. 2005] Scaffardi L. B., Pellegri N., de Sanctis O. and Tocho J. O., Sizing gold nanoparticles by optical extinction spectroscopy, Nanotechnology 16 (1), 158-163 (2005).

[Scaffardi et al. 2007] Scaffardi L. B., Lester M., Skigin D. and Tocho J. O., Optical extinction spectroscopy used to characterize metallic nanowires, Nanotechnology 18 (31), 315402-315409 (2007).

[Schinca and Scaffardi 2008] Schinca D. C. and Scaffardi L. B., Core and shell sizing of small silver coated nanospheres by optical extinction spectroscopy, Nanotechnology 19 (49), 495712-495719 (2008).

[Schinca et al. 2009] Schinca D. C., Scaffardi L. B., Videla F. A., Torchia G. A., Moreno P. and Roso L., Silver-silver oxide core-shell nanoparticles by femtosecond laser ablation: core and shell sizing by extinction spectroscopy, J. Phys. D: Appl. Phys. 42 (21), 215102-215110 (2009).

[Schmidt et al. 1996] Schmidt A. A., Offermann J. and Anton R., The role of neutral oxygen radicals in the oxidation of Ag films, Thin Solid Films 281-282 (1-2), 105-107 (1996). 
[Schott] http://www.schott.com/english/news/publications/catalog_glass. html.

[Shipway et al. 2000] Shipway A. N., Katz E. and Willner I., Nanoparticle Arrays on Surfaces for Electronic, Optical, and Sensor Applications, CHEMPHYSCHEM 1, 18-52 (2000).

[Shulz 1954] Shulz L. G., The Optical Constants of Silver, Gold, Copper and Aluminum. I. The Absorption Coefficient k, J. Opt. Soc. Am. 44 (5), 357-362 (1954).

[Shulz and Tangherlini 1954] Shulz L. G. and Tangherlini F. R., Optical Constants of Silver, Gold, Copper and Aluminum. II. The Index of Refraction n, J. Opt. Soc. Am. 44 (5), 362-357 (1954).

[Schultz et al. 2000] Schultz S., Smith D. R., Mock J. J. and Schultz D. A., Single-target molecule detection with nonbleaching multicolor optical immunolabels, Proc. Natl. Acad. Sci. U.S.A. 97 (3), 996-1001 (2000).

[Smith et al. 1997] Smith D. F., Graybill G. R., Grubbs R. K. and Gucinski J. A., New developments in very high rate silver oxide electrodes, $J$. Power Sources 65 (1-2), 47-52 (1997).

[Sommerfeld 1899] Sommerfeld A., Über die Fortpflanzung elektrodynamischer Wellen längs eines Drahtes, Ann. Phys. und Chemie 67, 233-290 (1899).

[Stewart et al. 2008] Stewart M. E., Anderton C. R., Thompson L. B., Maria J., Gray S. K., Rogers J. A. and Nuzzo R. G., Nanostructured plasmonic sensors, Chem. Rev. 108 (2), 494-521 (2008). 
[Tam et al. 2004] Tam F., Moran C. and Halas N., Geometrical Parameters Controlling Sensitivity of Nanoshell Plasmon Resonances to Changes in Dielectric Environment, J. Phys. Chem. B 108 (45), 17290-17294 (2004).

[Thio et al. 2001] Thio T., Pellerin K. M., Linke R. A., Lezec H. J. and Ebbesen T. W., Enhanced light transmission through a single subwavelength aperture, Optics Letters 26 (24), 1972-1974 (2001).

[Tilaki et al. 2007] Tilaki R. M., Iraji Zad A. and Mahdavi S. M., Size, composition and optical properties of copper nanoparticles prepared by laser ablation in liquids, Appl. Phys. A 88 (2), 415-419 (2007).

[Torchia et al. 2008] Torchia G. A., Scaffardi L. B., Méndez C., Moreno P., Tocho J. O. and Roso L., Optical extinction for determining size distribution of gold nanoparticles fabricated by ultrashort pulsed laser ablation, Appl. Phys. A: Material Science \& Processing 93 (4), 967-971 (2008).

[Valverde-Aguilar et al. 2008] Valverde-Aguilar G., García-Macedo J. A. and Rentería-Tapia V., Silver Core-Silver Oxide Shell Nanoparticles Embedded on Mesostructured Silica Films, J. Nano Res. 3, 103-104 (2008).

[Videla et al. 2009] Videla F. A., Torchia G. A., Schinca D. C., Scaffardi L. B., Moreno P., Méndez C., Roso L., Giovanetti L. J. and Ramallo López J., Role of supercontinuum in the fragmentation of colloidal gold nanoparticles in solution, Proceeding SPIE 74050, 74050 U1 a U12 (2009). 
[Videla et al. 2010] Videla F. A., Torchia G. A., Schinca D. C., Scaffardi L. B., Moreno P., Méndez C., Giovanetti L., Ramallo López J. and Roso L., Analysis of the main optical mechanisms responsible for fragmentation of gold nanoparticles by femtosecond laser radiation, $J$. Appl. Phys. 107, 114308-114315 (2010).

[Wang et al. 2004] Wang H., Huang Y., Tan Z. and Hu X., Fabrication and characterization of copper nanoparticle thin-films and the electrocatalytic behavior, Analytica Chimica Acta 526 (1), 13-17 (2004).

[Wood and Ashcroft 1982] Wood D. M. and Ashcroft N. W., Quantum size effects in the optical properties of small metallic particles, Phys. Rev. B 25 (10), 6255-6274 (1982).

[Wood 1902] Wood R. W., On a Remarkable Case of Uneven Distribution of Light in a Diffraction Grating Spectrum, Proc. Phys. Soc. London 18, 269-275 (1902).

[Xia et al. 2000] Xia Y., Gates B., Yin Y. and Lu Y., Monodispersed Colloidal Spheres: Old Materials with New Applications, Adv. Mater. 12 (10), 693-713 (2000).

[Yin et al. 2005] Yin M., Wu C.-K., Lou Y., Burda C., Koberstein J. T., Zhu Y. and O'Brien S., Copper Oxide Nanocrystals, J. Am. Chem. Soc. 127 (26), 9506-9511 (2005).

[Zenneck 1907] Zenneck J., Über die Fortpflanzung ebener elektromagnetischer Wellen längs einer ebenen Leiterfläche und ihre Beziehung zur drahtlosen Telegraphie, Ann. d Phys. 328 (10), 846-866 (1907). 
[Zhao et al. 2006] Zhao J., Das A., Zhang X., Schatz G. C., Sligar S. G. and Van Duyne R. P., Resonance Surface Plasmon Spectroscopy: Low Molecular Weight Substrate Binding to Cytochrome P450, J. Am. Chem. Soc. 128 (34), 11004-11005 (2006).

[Zhu et al. 2005] Zhu H., Zhang C. and Yin Y., Novel synthesis of copper nanoparticles: influence of the synthesis conditions on the particle size, Nanotechnology 16 (12), 3079-3083 (2005).

[Zou and Schatz 2006] Zou S. L. and Schatz G. C., Metal nanoparticle array waveguides: Proposed structures for subwavelength devices, Phys. Rev. B 74 (12), 125111 (2006).

[Zuo et al. 1999] Zuo J. M., Kim M., O'Keeffe M. and Spence J. C. H., Direct observation of d-orbital holes and $\mathrm{Cu}-\mathrm{Cu}$ bonding in $\mathrm{Cu}_{2} \mathrm{O}$, Nature 401 (6748), 49-52 (1999). 


\section{Agradecimientos}

Es muy complicado resumir en pocas líneas todos los buenos recuerdos y la mezcla de sensaciones que en este momento se pasan por mi cabeza y corazón.

En todo este tiempo de estudio e investigación he recibido una cantidad innumerable de apoyo, consejo, información, estímulo, enseñanza, buena compañía, experiencia, discusión, crítica y vivencia, que me han acompañado tanto en mi vida personal como en mi formación académica, permitiendo la realización y finalización de este trabajo de Tesis. Creí oportuno ocupar un espacio para poder agradecer a aquellas personas que transitaron conmigo este largo viaje, con mayor o menor intensidad.

En primer lugar, quiero decir un enorme e infinito ¡GRACIAS! a mi familia, sin ellos no hubiera sido posible empezar, transitar y seguramente terminar este viaje. Empezando por mis padres Noemí y José, sin el apoyo de ellos nada de esto hubiera sido posible, nada. A ellos les debo la persona que soy. Gracias por haberme brindado todas las facilidades que tuvieron a su alcance durante mi etapa de estudiante y doctorando. A vos mi modelo de hija, hermana, tía, esposa, abuela, mujer y madre maravillosa, que desde que estuvimos en tu vientre nos diste tu amor incondicional. No tengo más que palabras de agradecimiento por estar siempre, en todos los momentos de mi vida, cerca o lejos, como nos ha tocado en estos últimos años. Gracias por 
alegrarte con todos mis aciertos, por acompañarme y tratar de animarme cuando me he sentido triste o desanimada, por soñar conmigo, por enorgullecerte por cada paso que di en mi vida, por tu gran apoyo y consejos, ¡MUCHAS GRACIAS MAMÁ! por eso y todo lo que haces por nosotros. Papá, estas son las líneas más difíciles que me ha tocado escribir, siento mucho dolor y tristeza, porque no hay nada que me hubiera gustado más que estuvieras a mi lado en este momento especial de mi vida y del cual estabas tan orgulloso. Te fuiste hace 1 año y 5 meses, y con lágrimas en mis ojos no quiero dejar de decirte ¡GRACIAS PAPÁ!, tu hija está a un paso de ser Doctora y sé que eso te va a poner muy feliz donde estés. A mis hermanos Anghy, Franco, Sole, Carlos y Daniel, gracias por haberme brindado tanto apoyo y amor constantemente. En especial quiero agradecerte a vos Anghy por tus esporádicas compañías, por tu apoyo y cariño incondicional, por prestar tu oído todas las veces que lo he necesitado, por tus cuidados cuando me he sentido enferma, por las largas charlas cuando no me podía dormir, ;MIL GRACIAS! hermanita. No puedo dejar de mencionar dos personitas maravillosas que han llegado a nuestra familia y nos han alegrado la vida, a ustedes Agustín y Valentín, gracias chiquitos hermosos por llenarme de felicidad cada vez que los veo, ojalá pudiera compartir más tiempo con ustedes.

Dicen que las oportunidades más importantes que pueden cambiar la vida de la gente surgen de la combinación exacta de estar precisa y simultáneamente en un determinado tiempo, lugar y con las personas indicadas. Ninguna otra situación es más explicativa que ésta, para describir el inicio de mi formación doctoral, que en dicha confluencia de fenómenos se destaca el haberme topado con mi directora Lucía Scaffardi y mi codirector Daniel Schinca. Ellos que no sólo son dos personas asombrosamente increíbles sino también investigadores admirables, siendo la combinación de ambas cualidades la que los hace muy especiales. Siempre 
les agradeceré por su eterna confianza, por darme también la oportunidad de transitar y concluir este tan preciado viaje, por aconsejarme constantemente acerca del próximo paso a seguir, por transformarse en padres y amigos, por haberme acompañado y guiado en todo momento, por las valiosas discusiones, por el ambiente agradable que generan a la hora de trabajar, por dejarme aprender de ustedes y brindarme su conocimiento, por el trato siempre respetuoso con una mezcla de afecto y humor, por su colaboración en eventos científicos, y por tantas cosas más, que en este momento no me vienen a la memoria pero que se las digo cada vez que se presenta la oportunidad, les estaré eternamente agradecida. Siempre estuvieron en todos los detalles, y cuando digo todos me refiero a todos. No me imagino cuál sería mi realidad profesional si no me hubiera acercado a Lucía ese 8 de agosto de 2007 en la Escuela Giambiagi para pedirle trabajar con ella y tratar de resolver con urgencia la documentación solicitada por el CONICET. Mi agradecimiento hacia usted se extiende nuevamente, por su rol de directora de esta Tesis, de madre y amiga.

Quiero agradecer a la dirección del Centro de Investigaciones Ópticas (CIOp, CONICET La Plata - CIC), particularmente a los Dres. Jorge O. Tocho y Marcelo R. Trivi por la oportunidad de realizar este trabajo de Tesis en la institución, y por haberme ayudado económicamente los primeros meses de mi llegada a la ciudad de La Plata.

Agradezco a todo el personal del CIOp, investigadores, personal de apoyo, becarios, técnicos y administrativos. Su amabilidad y ayuda siempre facilitaron mi trabajo en la institución.

Sin lugar a dudas, no sólo quiero mencionar el apoyo financiero del Consejo Nacional de Investigaciones Científicas y Técnicas (CONICET), al otorgarme una Beca de Postgrado correspondiente al programa de becas para Áreas de Vacancia Geográfica (AVG), sino también a la Universidad Nacional de Catamarca (UNCa) y al Ministerio de Ciencia, Tecnología e 
Innovación Productiva (MinCyT) por formar parte de las instituciones responsables del Convenio de Cooperación del programa de Becas AVG. En este sentido, mi agradecimiento se extiende a la Facultad de Ciencias Exactas de la Universidad Nacional de La Plata (UNLP), por permitirme la inscripción al doctorado en tan prestigiosa Universidad.

Un agradecimiento especial, a la Dra. Marcela B. Fernández van Raap del Laboratorio de Microscopías de Fuerza Atómica y Magnética del Instituto de Física de La Plata (LMFAyM - IFLP), por su valioso tiempo, dedicación, colaboración y confianza, a la hora de observar y analizar las imágenes AFM, las cuales han sido de mucha importancia en este trabajo de Tesis.

También quiero agradecer a los proyectos: PIP 0394 del CONICET, 11/I151 de la Facultad de Ingeniería de la UNLP, PME 2006-0018 (ANPCyT) y al subsidio del MinCyT, sin esta ayuda económica este trabajo de Tesis no se hubiese podido desarrollar.

Continuando con los agradecimientos personales, no quiero olvidarme de mis tíos, tías, primos y primas maternos y paternos, en especial a mi tía Gringa por brindarme todo su cariño y estímulo para seguir adelante y a mi tío Rubén por prestar siempre su interés hacia mi trabajo, a ambos por estar orgullosos de su sobrina, igracias!.

Un especial agradecimiento a mis amigas y hermanas del alma de Catamarca, que siempre han estado presentes de alguna manera en esta etapa de mi vida y a las cuales extraño un montón, Auro, Meli, Pao, Gaby, Lenny, Noe, Erica, Marcia, Angelita, Elizabeth y Carmen, espero no olvidarme de ninguna, aunque seguro eso me pase. Auro, estoy eternamente agradecida por todas tus hermosas palabras de aliento y por tu cariño incondicional, gracias por tus visitas sorpresas y también gracias a tu familia por el afectuoso recibimiento que siempre me dan. 
Quiero destacar mi infinito agradecimiento a unas personas maravillosas que han llegado a mi vida en diferentes momentos de estos 4 años y 9 meses, formando verdaderas amistades, ellos son: Fabián, Eri, Marthita, Angela, Lore, Vale, Angélica, Andrés, Fede, Jonh F., Flavia, Martin, Emanuel, Laureano, Patricio, Daniel, Roxana, Ceci, Vane y Adriana. Encontré gente increíble, generosa, siempre dispuesta a dar lo mejor de sí, de la que ya no abunda, he pasado momentos inolvidables con ustedes. Agradezco a los primeros que estuvieron por su apoyo. A los que caminaron conmigo la senda del doctorado, por los momentos compartidos y las jugosas conversaciones. A los más nuevos por la buena onda. Pero no puedo dejar de hacer una mención o agradecimiento especial a las personitas que han estado presentes en las buenas y en las malas, ¡MUCHAS GRACIAS! Fabián, Eri, Fede, Angela, Lore, Angélica, Andrés, Daniel y Flavia, por sus palabras, su compañía, su alegría, los momentos inolvidables, y todo lo que me han brindado. Por haberme ayudado con pequeñas cuestiones que han llevado a poder construir un todo, como esta Tesis.

Fabián V., compañero como olvidar de agradecerte, todos los buenos gestos de humildad y generosidad que has tenido conmigo, ha sido un placer enorme haber trabajado a tu lado, gracias por tu paciencia, por las productivas discusiones que hemos tenido durante este arduo trabajo de investigación y por tus interesantes aportes.

Dafne, gracias por haberme abierto las puertas de tu casa en el comienzo de mi segundo año en La Plata y por brindarme tu amistad.

Agradezco también al Dr. Marcelo Trivi, por su constante colaboración, motivación, apoyo e inclusión de los becarios y/o jóvenes investigadores a diferentes actividades que atañen al CIOp, lo cual es un gesto muy valorable y que no es común encontrar en este ámbito. 
Por último quiero agradecer a los miembros del jurado que se han elegido para evaluar este trabajo de Tesis, por todas las apreciaciones y reflexiones que seguramente se transformarán en elementos constructivos de futuras investigaciones sobre este tema.

Probablemente, me haya olvidado de muchas otras personas que se relacionan directamente con aportes $\mathrm{u}$ otro tipo de colaboraciones para el desarrollo de esta Tesis.

Igualmente, les agradezco a ustedes y a todos los anteriormente mencionados por que de alguna $u$ otra manera han colaborado en la producción de esta Tesis, contando con el gran apoyo realizado desinteresadamente por parte de mucha gente. 